\title{
Targeting bile salt-FGF19 signaling
}

Citation for published version (APA):

Chang, X. (2021). Targeting bile salt-FGF19 signaling: promising therapeutic strategies to promote liver regeneration and improve intestinal failure. [Doctoral Thesis, Maastricht University]. Maastricht University. https://doi.org/10.26481/dis.20211026xc

Document status and date:

Published: 01/01/2021

DOI:

10.26481/dis.20211026xc

Document Version:

Publisher's PDF, also known as Version of record

\section{Please check the document version of this publication:}

- A submitted manuscript is the version of the article upon submission and before peer-review. There can be important differences between the submitted version and the official published version of record.

People interested in the research are advised to contact the author for the final version of the publication, or visit the DOI to the publisher's website.

- The final author version and the galley proof are versions of the publication after peer review.

- The final published version features the final layout of the paper including the volume, issue and page numbers.

Link to publication

\footnotetext{
General rights rights.

- You may freely distribute the URL identifying the publication in the public portal. please follow below link for the End User Agreement:

www.umlib.nl/taverne-license

Take down policy

If you believe that this document breaches copyright please contact us at:

repository@maastrichtuniversity.nl

providing details and we will investigate your claim.
}

Copyright and moral rights for the publications made accessible in the public portal are retained by the authors and/or other copyright owners and it is a condition of accessing publications that users recognise and abide by the legal requirements associated with these

- Users may download and print one copy of any publication from the public portal for the purpose of private study or research.

- You may not further distribute the material or use it for any profit-making activity or commercial gain

If the publication is distributed under the terms of Article $25 \mathrm{fa}$ of the Dutch Copyright Act, indicated by the "Taverne" license above, 
Targeting bile salt-FGF19 signaling: promising therapeutic strategies to promote liver regeneration and improve intestinal failure 
(C) Xinwei Chang, Maastricht 2021

All rights are reserved. For articles published, the copyright has been transferred to the respective publisher. No parts of this thesis may be reproduced, stored in a retrieval system or transmitted in any form or by any means, without prior permission from the author.

Cover design: Xinwei Chang

Printed by: PRINTSUPPORT4U

ISBN: 978-94-92597-82-3 


\title{
Targeting bile salt-FGF19 signaling: promising therapeutic strategies to promote liver regeneration and improve intestinal failure
}

\author{
Dissertation \\ to obtain the degree of Doctor at Maastricht University, \\ on the authority of Rector Magnificus, \\ Prof. dr. Rianne M. Letschert, \\ in accordance with the decision of the Board of Deans, \\ to be defended in public on \\ Tuesday October $26^{\text {th }}, 2021$ at 13:00 hours
}

by

Xinwei Chang 


\section{Supervisor}

Prof. dr. Steven W.M. Olde Damink

\section{Co-supervisior}

Dr. Frank G. Schaap

\section{Assessment Committee}

Prof. dr. Nicole Bouvy (Chairman)

Prof. dr. Frederik-Jan Van Schooten

Dr. Geert Wanten (Radboud University Medical Centre, The Netherlands)

Dr. Marc Bemelmans

Prof. dr. Thorsten Cramer (RWTH Aachen University, Germany) 


\section{Contents}

Chapter 1 General introduction, aims, and outline of the thesis

Part I Preoperative biliary drainage in perihilar cholangiocarcinoma

Chapter 2 The effect and route of preoperative biliary drainage in patients with resectable perihilar cholangiocarcinoma: a systematic review of systematic reviews

Submitted

Part II The role of bile salt signaling in liver regeneration

Chapter 3 Precision-cut liver slices as a model to study human liver regeneration

Chapter 4 Obeticholic acid accelerates portal vein embolization-induced liver hypertrophy by improving bile salt homeostasis in rabbits Manuscript in preparation

Chapter 5 No influence of cholestasis on portal vein embolization-induced hypertrophy of the future liver remnant

Submitted

Part III Restored bile salt-FGF19 signaling by chyme reinfusion in intestinal failure

Chapter 6 Chyme reinfusion restores the regulatory bile salt-FGF19 axis in intestinal failure patients

Hepatology 2021 Jun 16 (Published)

Chapter 7 General Discussion

$\begin{array}{lll}\text { Appendix I Summary } & 213\end{array}$

$\begin{array}{lll}\text { Appendix II Impact } & 221\end{array}$

$\begin{array}{lll}\text { Appendix III Acknowledgements } & 229\end{array}$

$\begin{array}{lll}\text { Appendix IV Curriculum Vitae } & 235\end{array}$

Appendix V List of Publications 239 



\section{Chapter 1}

General introduction 



\section{Introduction}

Bile salts are synthesized in the hepatocytes from cholesterol through a complex series of biochemical reactions 1,2 . The initial and rate-determining step in the main biosynthetic route (i.e. the classic pathway) is catalyzed by the enzyme cholesterol 7alpha-hydroxylase CYP7A $1{ }^{1}$. The end products of the biosynthetic pathways, i.e. bile salts, are conjugated with the amino acid glycine or taurine and secreted into the canalicular lumen via the bile salt export pump (BSEP) ${ }^{3}$. Bile and its constituent bile salts are transported through a ductular network also comprising the gallbladder, to the proximal small intestine (duodenum). In the terminal ileum, bile salts are reabsorbed by enterocytes and return to the liver through the portal circulation, completing the enterohepatic circulation (EHC) (Fig. 1).



Figure 1. Enterohepatic circulation of bile salts. Bile salts are synthesized in the hepatocytes from cholesterol and conjugated with the amino acid glycine or taurine, and secreted into the canalicular lumen. In the terminal ileum, $95 \%$ of bile salts are reabsorbed by enterocytes and return to the liver through the portal circulation (red arrow). Remaining $5 \%$ of bile salts are lost in the feces (blue arrow). (This illustration is adapted from BioRender [https://biorender.com/]).

During enterohepatic circulation, bile salts activate dedicated receptors (e.g. farnesoid $X$ receptor, FXR; G protein-coupled bile acid receptor, TGR5) in the terminal ileum and liver ${ }^{3}$. Activation of bile salt receptors plays important roles in hepatic bile salt 
homeostasis, hepatic nutrient metabolism in the postprandial state, small intestinal barrier function, and inflammatory control ${ }^{3}$. Hence, an intact enterohepatic circulation of bile salt fulfills an important role in gut-liver health ${ }^{3,4}$.

Surgical patients, especially patients with hepatobiliary and pancreatic diseases, often suffer from a compromised EHC, which has detrimental effects on gut-liver health 5 . For examples, patients with perihilar cholangiocarcinoma ( $\mathrm{PCCA}$ ) or head of pancreas tumors have an obstructed EHC, because of malignant obstruction and/or compression of the bile duct. These patients present with obstructive jaundice and elevated systemic markers of cholestatic liver injury ${ }^{6}$. Furthermore, for patients who underwent a partial hepatectomy, especially when accompanied by concomitant removal of the gallbladder during extended resection procedures, the EHC can be temporarily disrupted due to removal of the 'source' of bile salts. Major liver resection can lead to post-hepatectomy liver failure (PHLF) and death, especially in patients with $\mathrm{pCCA}^{7}$. In addition, patients with a temporary double enterostomy in the jejunum have a disrupted EHC due to outflow of succus entericus in the stoma bag ${ }^{8}$. They can develop intestinal failure (IF) and intestinal failure-associated liver disease ${ }^{8}$. To understand the pathophysiology of EHC disturbances in these disease contexts further research is needed, ultimately aiming at targeting affected signaling pathways to improve patient outcomes.

\section{Biliary obstruction and drainage}

\section{Disturbed enterohepatic circulation}

Under biliary obstruction (e.g. pCCA or head of pancreas tumors), the EHC of bile salts is disturbed. Bile fails to flow towards the intestine. Under these conditions, the transport of bile salts in and out of hepatocytes can undergo adaptive changes. $\mathrm{Na}^{+}-$ taurocholate cotransporting polypeptide (NTCP, mediating uptake of bile salts from the circulation) is downregulated, while basolateral organic solute transporter a/ $\beta$ (OSTa/ $\beta$ ) and multidrug resistance-associated protein 3 (MRP3) and MRP4 are upregulated, thereby releasing bile salts into the sinusoidal circulation ${ }^{9,10}$. The elevated expression of BSEP (bile salt exporter) can result in an unfavored increase of biliary pressure ${ }^{11,}$ 12. Impaired canalicular bile salt secretion and obstructed bile flow can lead to accumulation of bile salts in hepatocytes and attendant hepatotoxicity ${ }^{3}$. 
Cholestatic influences on cholangiocytes and hepatocytes

Biliary obstruction induces adaptive changes in the biliary tree. The canalicular network undergoes volumetric expansion by an increase in diameter, with increased number of spine-like or bleb-like protrusions intruding into the hepatocyte cytoplasm ${ }^{10}$. In the bile duct ligation (BDL) model of cholestasis, the disruption of tight junctional barrier and the periductular cytoskeleton, may explain the change of canalicular morphology and leakage of bile into the parenchyma ${ }^{10}$. Additionally, increased hydrostatic pressure in the biliary tree, which is dependent on the presence of BSEP, may lead to liver injury after BDL ${ }^{13}$.

Elevated intracellular bile salt concentrations can lead to hepatocyte injury through various mechanism. Bile salts can stimulate the mitochondrial respiratory chain to generate reactive oxygen species, which can damage mitochondrial membranes and promote the release of apoptosis-inducing factor, ultimately leading to hepatocyte apoptosis ${ }^{14}$. The biological and physicochemical properties of bile salts are determined by the ionization of the side chain carboxylic acid group, and by the number, position and spatial orientation of hydroxyl groups in the steroid nucleus ${ }^{15}$ (Fig. 2). In general, hydrophobic bile salts are more toxic because of increased prospensity to disrupt membrane integrity by solubilizing membrane lipids ${ }^{15}$. Hydrophobic bile salts can also induce endoplasmic reticulum (ER) stress, subsequently activate c-Jun Nterminal kinase (JNK) signaling and, thus, contributing to hepatocyte cell death in the context of cholestasis ${ }^{16}$. Death receptors are another factor mediating cholestasisrelated hepatocyte apoptosis ${ }^{17}$.



Figure 2. Chemical structure of primary bile salt cholic acid. In general, bile salts have a steroid nucleus containing one or multiple hydroxyl groups, and a side chain containing a carboxyl group. This illustration is a modification from a picture of Hofmann et al. (2008) ${ }^{18}$. 
Inflammation

Bile salt toxicity induces cell death and an attendant inflammatory response 19 . Neutrophils are the predominant infiltrating immune cells in the early stages of cholestatic liver injury ${ }^{20}$. The periportal neutrophil infiltration is positively correlated with the degree of liver injury in BDL mice and cholestatic patients ${ }^{20,21}$. Macrophages also participate in cholestatic liver injury. In pathological concentration, extracellular bile salts act as a danger signal to activate the NLRP3 inflammasome in macrophages 22. Treatment with gadolinium chloride, an inhibitor of Kupffer cell phagocytic function, could ameliorate liver injury and fibrosis in BDL rats ${ }^{23}$.

Preoperative biliary drainage

Due to above harmful effects of biliary obstruction, preoperative biliary drainage (BD) is commonly used to decompress the biliary tree and restore $\mathrm{EHC}$ in patients with obstructive jaundice ${ }^{24,25}$. Through internal BD, the natural route of bile flow towards the small intestine is restored. Gene expression profiling revealed that BD improved cholestasis-associated fibrogenic and inflammatory gene signatures in the liver of patients with $\mathrm{pCCA}{ }^{26}$. Preoperative $\mathrm{BD}$ was associated with decreased mortality in patients with pCCA after liver resection ${ }^{27}$. However, preoperative BD can lead to drainage-related complications such as cholangitis, pancreatitis and haemobilia ${ }^{28}$. Some studies do not recommend preoperative BD for pCCA, due to lack of benefits on reducing postoperative complications and mortality 29,30 . Currently, there is no consensus on the routine use of, and indications for preoperative BD in patients with resectable pCCA.

Percutaneous transhepatic biliary drainage (PTBD) and endoscopic biliary drainage (EBD) are the two main drainage procedure (Fig. 3). The best preoperative drainage procedure in resectable pCCA is under debate ${ }^{31-35}$. PTBD is relatively invasive and may be associated with seeding metastasis ${ }^{31}$. In addition, some studies reported poor postoperative survival in patients undergoing PTBD ${ }^{31,36}$. EBD is performed by a retrograde route from the ampulla of Vater in the proximal small intestine to the (intrahepatic) bile duct, which avoids the potential disadvantages of PTBD. However, as EBD causes a direct connection between intestinal microbes and the biliary system, it can result in ascending cholangitis, a risk for postoperative mortality ${ }^{25}$. The first randomized controlled trial that compared PTBD with EBD in resectable pCCA was prematurely stopped because of higher all-cause mortality in the PTBD group ${ }^{37}$. A 
possible explanation is that external drainage of bile results in intestinal bile salt deficiency and accordingly insufficient enterohepatic bile salt signaling, which negatively affects liver regeneration ${ }^{37}$.


Figure 3. Preoperative biliary drainage in perihilar cholangiocarcinoma. Percutaneous transhepatic biliary drainage (PTBD) is performed through punctures of the skin into the intrahepatic bile duct (left panel). Endoscopic biliary drainage (EBD) is achieved by retrograde route via the proximal small intestine and biliary tract (right panel). This illustration is a modification from a picture of Tavakkoli et al. (2021) ${ }^{38}$.

\section{Bile salt homeostasis and liver regrowth}

\section{Bile salt-FGF19 signaling}

Partial hepatectomy $(\mathrm{PH})$ for hepatobiliary tumors is relatively safe and often the only curative treatment option. The robust capacity of the liver to regenerate permits up to $70 \%$ removal of the liver mass ${ }^{39}$. However, after (extended) partial hepatectomy, especially when accompanied by cholecystectomy, the EHC can be disrupted due to the loss of the 'source' of bile salts. Bile salts are involved in liver regeneration after $\mathrm{PH}$ via activation of the FXR ${ }^{40}$. FXR activation in the ileum stimulates the expression of fibroblast growth factor 19 (FGF19, Fgf15 in rodents) in enterocytes ${ }^{41}$. After binding to the fibroblast growth factor receptor 4 (FGFR4) and $\beta$-Klotho (KLB) complex at the surface of hepatocytes, FGF19 inhibits synthesis of bile salts via suppressing the expression of CYP7A1 ${ }^{41}$ (Fig. 4). FGF19 also exerts mitogenic properties through activation of FGFR4 signaling ${ }^{42}$. Delayed liver regrowth and high mortality was observed in Fxr or Fgf15 deficient mice after $\mathrm{PH}$, which is linked with bile salt toxicity 40,43 . In patients undergoing $\mathrm{PH}$, serum bile salt levels were positively associated with liver regeneration ${ }^{44}$. 




Figure 4. Enterohepatic bile salt-FGF19 signaling. In the ileum, bile salts bind and activate nuclear receptor farnesoid $X$ receptor (FXR) and this stimulates the expression of fibroblast growth factor 19 (FGF19). In the liver, FGF19 binds to its receptor fibroblast growth factor receptor 4 (FGFR4), which activates a downstream signaling pathway involving MAP kinases that causes repression of CYP7A1, thus suppressing bile salt synthesis. This illustration is a modification from a picture of Schaap et al. (2014) ${ }^{3}$.

Liver regrowth induced by portal vein embolization

For a safe liver resection, the future liver remnant (FLR) volume must be $>30 \%$ in uncompromised livers. However, liver resections are commonly performed in patients with underlying liver disease. Portal vein embolization (PVE) is the gold standard procedure to increase the FLR volume in advance of major hepatectomy ${ }^{45}$. PVE induces hypertrophy of the FLR by depriving the embolized segments of nutrients, which provides a trigger for compensatory expansion of the FLR ${ }^{46}$. Moreover, PVE treatment can increase circulating interleukin-6 (IL-6), hepatocyte growth factor (HGF), transforming growth factor- $\alpha$ (TGF- $\alpha$ ), and epidermal growth factor (EGF), which stimulate hepatic DNA synthesis and hepatocellular proliferation in rats ${ }^{46}$. In patients, PVE can induce an increase in FLR volume of $20-50 \%$ within $3-7$ weeks ${ }^{47}$. Studies have shown that PVE has a beneficial effect on decreasing liver failure and death rates in patients undergoing major liver resection ${ }^{48,49}$. However, relatively long waiting time between PVE and resection puts patients at risk of tumor progression ${ }^{50}$. In addition, 
PVE may induce insufficient hypertrophy of FLR to allow safe resection. Therefore, identifying factors affecting the proliferative effect of PVE is needed.

Bile salt signaling may play a role in the liver growth induced by PVE. After PVE, plasma bile salt levels were rapidly increased in both humans and in experimental PVE 51, 52. Bile salt concentrations at day 3 predict FRL volume increase at day 7 in a standardized rabbit model of PVE ${ }^{51}$. Furthermore, bile salt levels at $5 \mathrm{~h}$ after PVE were positively correlated with hypertrophy of the FLR and liver function in patients with CRLM 52. We recently demonstrated that obeticholic acid (OCA), a first-in-class selective and potent agonist of FXR, augments the volume gain and function of the non-embolized liver lobe in a rabbit model of PVE ${ }^{53}$. The underlying mechanisms remain unknown.

\section{Double jejunal enterostomy and chyme reinfusion}

Interrupted enterohepatic circulation in double jejunal enterostomy

In the enterohepatic circulation, bile salts exert direct antimicrobial activity through disrupting cell membrane integrity ${ }^{54,55}$. Indirectly, bile salts can induce Paneth cell production of antimicrobial peptides to inhibit gut microbial overgrowth through FXRdependent manners ${ }^{54,55}$. The interaction between gut microbiota and bile salts is mutual. The gut microbiota can deconjugate and further metabolize primary bile salts into secondary bile salts, thereby affecting their biological and signaling properties 55 . During digestive surgery, a number of clinical (emergency) situations may lead to the establishment of a temporary double enterostomy in the jejunum, including small bowel resection and related anastomosis protection, peritonitis and enterocutaneous fistula 8. This leads to an interrupted EHC of bile salts due to leakage of the succus entericusin the stoma bag, rather than entering the (terminal) ileum. A double jejunal enterostomy can, thus, disturb the FXR/FGF19 feedback loop that controls hepatic bile salt synthesis, while bile salt deficiency in the distal small intestine can lead to intestinal dysbiosis ${ }^{54}$. In mice, secondary bile salt deficiency, due to the loss of contact between bile salt-metabolizing bacteria in the colon and bile salt substrates, can promote intestinal inflammation by reduced anti-inflammatory signaling through bile salt receptor Tgr5 in immune cells ${ }^{56}$. Compromised gut barrier allows microorganisms and microorganism-derived products to translocate to the liver, causing liver inflammation 
and subsequent immune-mediated injury ${ }^{54}$.

Chyme reinfusion

Chyme reinfusion $(C R)$ is an enteral nutritional technique, which re-establishes the anatomical continuity of the small intestine in patients with double jejunal enterostomy, through extracorporeal circulation of chyme, likely leading to restoration of the $\mathrm{EHC}$ of bile salts as well ${ }^{8}$. Previous studies have shown that reinfusion of proximal intestinal secretions normalized serum bilirubin, and levels of cholestatic injury markers alkaline phosphatase (ALP) and y-glutamyl transferase (GGT) in patients with high-output stomas or fistulas ${ }^{57,58}$. Furthermore, CR can increase plasma citrulline levels, a marker for the absorptive function of the remnant small intestine, in adult IF patients with a double enterostomy ${ }^{8}$. After CR initiation, 126 of 139 patients (91\%) could be weaned of parenteral nutrition within a median of 2 days ${ }^{8}$. Despite these promising findings, the mechanisms underlying the beneficial effects of CR are incompletely understood. But it has been postulated that bile salt signaling is involved ${ }^{8}$.

\section{Aims and outline of this thesis}

In this thesis, we 1) summarize the current knowledge of the effects and route of preoperative biliary drainage in patients with resectable pCCA, 2) a. explore the suitability of precision-cut liver slices (PCLS) as an ex vivo model to study liver regeneration, b. study the mechanisms underlying OCA-accelerated liver regrowth following PVE in a rabbit model, c. investigate the influence of cholestasis on PVEinduced FLR hypertrophy in patients, and 3) explore a contribution of the bile saltFGF19 signaling axis in beneficial effects of CR in intestinal failure patients.

In Part I, a systematic review of systematic reviews is conducted to assess the effect of preoperative $\mathrm{BD}$ versus no preoperative $\mathrm{BD}$ and to evaluate the superiority of $\mathrm{EBD}$ versus PTBD on perioperative and long-term outcomes in patients with resectable pCCA (Chapter 2). In Part II, we describe a model, precision-cut human liver slices (PCLS), as an ex vivo tool to study interventions aimed at promoting liver growth (Chapter 3). Further, the mechanisms underlying OCA-accelerated hypertrophy of non-embolized liver lobe is examined in a standardized rabbit model of PVE (Chapter 4). The findings from this animal study led us to investigate the influence of cholestasis on FLR growth in clinical patients who underwent PVE. We compared PVE-induced 
hypertrophy of the FLR in patients with CRLM (non-cholestatic) and pCCA (cholestatic or post-cholestatic) and analyzed the effect of different sides of biliary drainage (unilateral or bilateral) on FLR hypertrophy (Chapter 5). In Part III, it is investigated whether beneficial effects of chyme reinfusion in intestinal failure patients with a temporary double enterostomy, are in part mediated by the bile salt-FGF19 signaling axis (Chapter 6). In Chapter 7 key findings from Part I, Part II, and Part III are summarized and integrally discussed, along with potential clinical implications and future research directions. 


\section{Chapter 1}

\section{References}

1. Russell DW. The enzymes, regulation, and genetics of bile acid synthesis. Annu Rev Biochem. 2003;72:137-74.

2. Hofmann AF, Hagey LR. Key discoveries in bile acid chemistry and biology and their clinical applications: history of the last eight decades. J Lipid Res. 2014;55:1553-95.

3. Schaap FG, Trauner M, Jansen PL. Bile acid receptors as targets for drug development. Nat Rev Gastroenterol Hepatol. 2014;11:55-67.

4. Albillos A, de Gottardi A, Rescigno M. The gut-liver axis in liver disease: Pathophysiological basis for therapy. J Hepatol. 2020;72:558-77.

5. Hegyi P, Maléth J, Walters JR, Hofmann AF, Keely SJ. Guts and Gall: Bile Acids in Regulation of Intestinal Epithelial Function in Health and Disease. Physiol Rev. 2018;98:1983-2023.

6. Valle JW, Kelley RK, Nervi B, Oh DY, Zhu AX. Biliary tract cancer. Lancet. 2021;397:428-44.

7. van Mierlo KM, Schaap FG, Dejong CH, Olde Damink SW. Liver resection for cancer: New developments in prediction, prevention and management of postresectional liver failure. $\mathrm{J}$ Hepatol. 2016;65:1217-31

8. Picot D, Layec S, Dussaulx L, Trivin F, Thibault R. Chyme reinfusion in patients with intestinal failure due to temporary double enterostomy: A 15-year prospective cohort in a referral centre. Clin Nutr. 2017;36:593-600.

9. Geier A, Wagner M, Dietrich CG, Trauner M. Principles of hepatic organic anion transporter regulation during cholestasis, inflammation and liver regeneration. Biochim Biophys Acta. 2007;1773:283-308.

10. Jansen PL, Ghallab A, Vartak N, Reif R, Schaap FG, Hampe J, et al. The ascending pathophysiology of cholestatic liver disease. Hepatology. 2017;65:722-38.

11. Slitt AL, Allen K, Morrone J, Aleksunes LM, Chen C, Maher JM, et al. Regulation of transporter expression in mouse liver, kidney, and intestine during extrahepatic cholestasis. Biochim Biophys Acta. 2007;1768:637-47.

12. Schaap FG, van der Gaag NA, Gouma DJ, Jansen PL. High expression of the bile salthomeostatic hormone fibroblast growth factor 19 in the liver of patients with extrahepatic cholestasis. Hepatology. 2009;49:1228-35

13. van Golen RF, Olthof PB, Lionarons DA, Reiniers MJ, Alles LK, Uz Z, et al. FXR agonist obeticholic acid induces liver growth but exacerbates biliary injury in rats with obstructive cholestasis. Sci Rep. 2018;8:16529.

14. Yerushalmi B, Dahl R, Devereaux MW, Gumpricht E, Sokol RJ. Bile acid-induced rat hepatocyte apoptosis is inhibited by antioxidants and blockers of the mitochondrial permeability transition. Hepatology. 2001;33:616-26.

15. Heuman DM. Quantitative estimation of the hydrophilic-hydrophobic balance of mixed bile salt solutions. J Lipid Res. 1989;30:719-30.

16. Marra F, Svegliati-Baroni G. Lipotoxicity and the gut-liver axis in NASH pathogenesis. J Hepatol. 2018;68:280-95

17. Wei S, Ma X, Zhao Y. Mechanism of Hydrophobic Bile Acid-Induced Hepatocyte Injury and Drug Discovery. Front Pharmacol. 2020;11:1084.

18. Hofmann AF, Hagey LR. Bile acids: chemistry, pathochemistry, biology, pathobiology, and therapeutics. Cell Mol Life Sci. 2008;65:2461-83.

19. Schubert K, Olde Damink SWM, von Bergen M, Schaap FG. Interactions between bile salts, gut 
microbiota, and hepatic innate immunity. Immunol Rev. 2017;279:23-35.

20. de Vries FEE, Reeskamp LF, van Ruler O, van Arum I, Kuin W, Dijksta G, et al. Systematic review: pharmacotherapy for high-output enterostomies or enteral fistulas. Aliment Pharmacol Ther. 2017;46:266-73.

21. Cai SY, Ouyang X, Chen Y, Soroka CJ, Wang J, Mennone A, et al. Bile acids initiate cholestatic liver injury by triggering a hepatocyte-specific inflammatory response. $\mathrm{JCl}$ Insight. 2017;2:e90780.

22. Hao H, Cao L, Jiang C, Che Y, Zhang S, Takahashi S, et al. Farnesoid X Receptor Regulation of the NLRP3 Inflammasome Underlies Cholestasis-Associated Sepsis. Cell Metab. 2017;25:85667 e5.

23. Zandieh A, Payabvash S, Pasalar P, Morteza A, Zandieh B, Tavangar SM, et al. Gadolinium chloride, a Kupffer cell inhibitor, attenuates hepatic injury in a rat model of chronic cholestasis. Hum Exp Toxicol. 2011;30:1804-10.

24. Inamdar S, Slattery E, Bhalla R, Sejpal DV, Trindade AJ. Comparison of Adverse Events for Endoscopic vs Percutaneous Biliary Drainage in the Treatment of Malignant Biliary Tract Obstruction in an Inpatient National Cohort. JAMA Oncol. 2016;2:112-7.

25. Lidsky ME, Jarnagin WR. Surgical management of hilar cholangiocarcinoma at Memorial Sloan Kettering Cancer Center. Ann Gastroenterol Surg. 2018;2:304-12.

26. Reiniers MJ, de Haan L, Weijer R, Wiggers JK, Jongejan A, Moerland PD, et al. Effect of preoperative biliary drainage on cholestasis-associated inflammatory and fibrotic gene signatures in perihilar cholangiocarcinoma. Br J Surg. 2019;106:55-8.

27. Kennedy TJ, Yopp A, Qin Y, Zhao B, Guo P, Liu F, et al. Role of preoperative biliary drainage of liver remnant prior to extended liver resection for hilar cholangiocarcinoma. HPB (Oxford). 2009;11:445-51.

28. Hameed A, Pang T, Chiou J, Pleass H, Lam V, Hollands M, et al. Percutaneous vs. endoscopic pre-operative biliary drainage in hilar cholangiocarcinoma - a systematic review and metaanalysis. HPB 2016;18:400-10.

29. Ratti F, Cipriani F, Fiorentini G, Hidalgo Salinas C, Catena M, Paganelli M, et al. Management of hilum infiltrating tumors of the liver: The impact of experience and standardization on outcome. Dig Liver Dis. 2019;51:135-41.

30. Ramanathan R, Borrebach J, Tohme S, Tsung A. Preoperative Biliary Drainage Is Associated with Increased Complications After Liver Resection for Proximal Cholangiocarcinoma. J Gastrointest Surg. 2018;22:1950-7.

31. Komaya K, Ebata T, Yokoyama Y, Igami T, Sugawara G, Mizuno T, et al. Verification of the oncologic inferiority of percutaneous biliary drainage to endoscopic drainage: A propensity score matching analysis of resectable perihilar cholangiocarcinoma. Surgery. 2017;161:394-404.

32. Zhang XF, Beal EW, Merath K, Ethun CG, Salem A, Weber SM, et al. Oncologic effects of preoperative biliary drainage in resectable hilar cholangiocarcinoma: Percutaneous biliary drainage has no adverse effects on survival. J Surg Oncol. 2018;117:1267-77.

33. Kim KM, Park JW, Lee JK, Lee KH, Lee KT, Shim SG. A Comparison of Preoperative Biliary Drainage Methods for Perihilar Cholangiocarcinoma: Endoscopic versus Percutaneous Transhepatic Biliary Drainage. Gut Liver. 2015;9:791-9.

34. Jo JH, Chung MJ, Han DH, Park JY, Bang S, Park SW, et al. Best options for preoperative biliary drainage in patients with Klatskin tumors. Surg Endosc. 2017;31:422-9. 


\section{Chapter 1}

35. Walter T, Ho CS, Horgan AM, Warkentin A, Gallinger S, Greig PD, et al. Endoscopic or percutaneous biliary drainage for Klatskin tumors? J Vasc Interv Radiol. 2013;24:113-21.

36. Hirano S, Tanaka E, Tsuchikawa T, Matsumoto J, Kawakami H, Nakamura T, et al. Oncological benefit of preoperative endoscopic biliary drainage in patients with hilar cholangiocarcinoma. $\mathrm{J}$ Hepatobiliary Pancreat Sci. 2014;21:533-40.

37. Coelen RJS, Roos E, Wiggers JK, Besselink MG, Buis Cl, Busch ORC, et al. Endoscopic versus percutaneous biliary drainage in patients with resectable perihilar cholangiocarcinoma: a multicentre, randomised controlled trial. Lancet Gastroenterol Hepatol. 2018;3:681-90.

38. Tavakkoli A, Elmunzer BJ, Waljee AK, Murphy CC, Pruitt SL, Zhu H, et al. Survival analysis among unresectable pancreatic adenocarcinoma patients undergoing endoscopic or percutaneous interventions. Gastrointest Endosc. 2021;93:154-62.e5.

39. Forbes SJ, Newsome PN. Liver regeneration - mechanisms and models to clinical application. Nat Rev Gastroenterol Hepatol. 2016;13:473-85.

40. Huang W, Ma K, Zhang J, Qatanani M, Cuvillier J, Liu J, et al. Nuclear receptor-dependent bile acid signaling is required for normal liver regeneration. Science. 2006;312:233-6.

41. Inagaki T, Choi M, Moschetta A, Peng L, Cummins CL, McDonald JG, et al. Fibroblast growth factor 15 functions as an enterohepatic signal to regulate bile acid homeostasis. Cell Metab. 2005;2:217-25.

42. Niu J, Zhao J, Wu J, Qiao G, Gu J, Zhou C, et al. Curtailing FGF19's mitogenicity by suppressing its receptor dimerization ability. Proc Natl Acad Sci U S A. 2020;117:29025-34.

43. Uriarte I, Fernandez-Barrena MG, Monte MJ, Latasa MU, Chang HC, Carotti S, et al. Identification of fibroblast growth factor 15 as a novel mediator of liver regeneration and its application in the prevention of post-resection liver failure in mice. Gut. 2013;62:899-910.

44. Koelfat KVK, van Mierlo KMC, Lodewick TM, Bloemen JG, van der Kroft G, Amygdalos I, et al. Bile Salt and FGF19 Signaling in the Early Phase of Human Liver Regeneration. Hepatology Communications. 2021:12

45. Aloia TA. Associating Liver Partition and Portal Vein Ligation for Staged Hepatectomy: Portal Vein Embolization Should Remain the Gold Standard. JAMA Surg. 2015;150:927-8.

46. Yokoyama Y, Nagino M, Nimura Y. Mechanisms of hepatic regeneration following portal vein embolization and partial hepatectomy: a review. World J Surg. 2007;31:367-74.

47. Liu H, Zhu S. Present status and future perspectives of preoperative portal vein embolization. Am J Surg. 2009;197:686-90.

48. Olthof PB, Aldrighetti L, Alikhanov R, Cescon M, Groot Koerkamp B, Jarnagin WR, et al. Portal Vein Embolization is Associated with Reduced Liver Failure and Mortality in High-Risk Resections for Perihilar Cholangiocarcinoma. Ann Surg Oncol. 2020.

49. Olthof PB, Wiggers JK, Groot Koerkamp B, Coelen RJ, Allen PJ, Besselink MG, et al. Postoperative Liver Failure Risk Score: Identifying Patients with Resectable Perihilar Cholangiocarcinoma Who Can Benefit from Portal Vein Embolization. J Am Coll Surg. 2017;225:387-94.

50. Hoekstra LT, van Lienden KP, Doets A, Busch OR, Gouma DJ, van Gulik TM. Tumor progression after preoperative portal vein embolization. Ann Surg. 2012;256:812-7; discussion 7-8.

51. Hoekstra LT, Rietkerk M, van Lienden KP, van den Esschert JW, Schaap FG, van Gulik TM. Bile salts predict liver regeneration in rabbit model of portal vein embolization. J Surg Res. 2012;178:773-8. 
52. Hoekstra LT, van Lienden KP, Schaap FG, Chamuleau RA, Bennink RJ, van Gulik TM. Can plasma bile salt, triglycerides, and apoA-V levels predict liver regeneration? World J Surg. 2012;36:2901-8.

53. Olthof PB, Huisman F, Schaap FG, van Lienden KP, Bennink RJ, van Golen RF, et al. Effect of obeticholic acid on liver regeneration following portal vein embolization in an experimental model. Br J Surg. 2017;104:590-9.

54. Tripathi A, Debelius J, Brenner DA, Karin M, Loomba R, Schnabl B, et al. The gut-liver axis and the intersection with the microbiome. Nat Rev Gastroenterol Hepatol. 2018;15:397-411.

55. Wahlstrom A, Sayin SI, Marschall HU, Backhed F. Intestinal Crosstalk between Bile Acids and Microbiota and Its Impact on Host Metabolism. Cell Metab. 2016;24:41-50.

56. Sinha SR, Haileselassie Y, Nguyen LP, Tropini C, Wang M, Becker LS, et al. Dysbiosis-Induced Secondary Bile Acid Deficiency Promotes Intestinal Inflammation. Cell Host Microbe. 2020.

57. Gause CD, Hayashi M, Haney C, Rhee D, Karim O, Weir BW, et al. Mucous fistula refeeding decreases parenteral nutrition exposure in postsurgical premature neonates. J Pediatr Surg. 2016;51:1759-65.

58. Rinsema W, Gouma DJ, von Meyenfeldt MF, Soeters PB. Reinfusion of secretions from highoutput proximal stomas or fistulas. Surg Gynecol Obstet. 1988;167:372-6. 



\section{Part I}

Preoperative biliary drainage in perihilar cholangiocarcinoma 



\title{
Chapter 2
}

\begin{abstract}
The effect and route of preoperative biliary drainage in patients with resectable perihilar cholangiocarcinoma: a systematic review of systematic reviews
\end{abstract}

Xinwei Chang, Frank G. Schaap, Hongxia Shen, Cornelis H.C. Dejong, Bas Groot Koerkamp, Christiaan van der Leij, Ulf P. Neumann, Jos Kleijnen, Steven W.M. Olde Damink 


\section{Abstract}

Background: The beneficial effect, and route of preoperative biliary drainage (BD) via percutaneous transhepatic biliary drainage (PTBD) or endoscopic biliary drainage (EBD), in resectable perihilar cholangiocarcinoma ( $\mathrm{pCCA}$ ) are heavily debated. This study aimed to clarify the effect and route of preoperative BD on perioperative and long-term outcomes in patients with resectable pCCA.

Methods: PubMed, Embase, Cochrane Library, and KSR Evidence were searched from inception to March 31, 2020, to identify systematic reviews (SRs) with or without meta-analysis. Risk Of Bias In Systematic reviews (ROBIS) assessment tool was used.

Results: Eleven SRs with meta-analysis including 5950 patients were identified. All but one original studies in the SRs were retrospective and at risk of bias. Ten of eleven SRs had high risk of bias. For preoperative BD versus no preoperative BD, all SRs showed no statistical differences in postoperative mortality (odds ratios (ORs) from 0.70 to 1.06 ). Preoperative $B D$ was associated with increased postoperative major morbidity in 'simple criteria' patients receiving BD only based on the presence of jaundice (OR 1.57 95\% Cl 1.10-2.25). For EBD versus PTBD, three of four SRs showed that the postoperative mortality was not significantly different between two groups (ORs from 0.47 to 0.63 ). EBD was associated with higher drainage-related overall morbidity, cholangitis and pancreatitis rates in three of four, three of five, and four of four SRs, respectively (ORs from 2.23 to $3.13,4.58$ to $5.41,7.46$ to 11.52 , respectively). PTBD was associated with higher seeding metastasis rates and worse postoperative overall survival (ORs from 0.35 to 0.46 , hazard ratios (HR) $0.6795 \% \mathrm{Cl}$ 0.53-0.85, respectively).

Conclusion: This study does not end the debate on the significance and preferred route of preoperative BD. We showed that most available evidence has high risk of bias. The preoperative BD might need to be performed in strictly selected patients in terms of cholangitis, bilirubin levels and future liver remnant volume to avoid increased postoperative major morbidity. EBD might be associated with higher short-term drainage-related morbidity but more favorable long-term oncological outcomes. 


\section{Introduction}

Perihilar cholangiocarcinoma ( $\mathrm{pCCA}$ ) is the most common type of bile duct malignancy, accounting for $50 \%$ to $60 \%$ of all cholangiocarcinoma cases ${ }^{1,2}$. Some $90 \%$ of patients with pCCA clinically present with (painless) obstructive jaundice ${ }^{3}$. Surgery with complete resection represents the only curative opportunity, with approximately $75 \%$ of patients eligible for surgical resection ${ }^{4}$. However, the prognosis of patients after surgery remains poor and 5-year survival rates are around $30 \%{ }^{5}$. Moreover, liver resection in patients with hyperbilirubinemia and (resolved) cholangitis carries a high postoperative risk of severe complications and mortality ${ }^{6}$.

Preoperative biliary drainage $(\mathrm{BD})$ is employed to decompress the biliary tree, treat cholangitis, and improve (future remnant) liver function ${ }^{7,8}$. BD can be performed unilaterally or bilaterally, and principally drains the future liver remnant ${ }^{9}$. BD is performed via a percutaneous procedure (PTBD: percutaneous transhepatic biliary drainage) or an endoscopic biliary drainage (EBD) procedure. Studies have demonstrated that preoperative BD can reverse the cholestasis-associated pro-fibrotic and inflammatory status of the liver, and enhance the ability of the liver to regenerate 10, 11 . However, the value of preoperative BD in resectable pCCA is under debate. Some studies argued against the utility of preoperative BD, finding no benefits in reducing postoperative complications and mortality ${ }^{12-14}$. Patients receiving preoperative BD even had higher mortality after left hemi-hepatectomy ${ }^{10}$.

In addition, the optimal drainage route still needs to be determined. PTBD can lead to the diversion of bile, subsequent disturbed enterohepatic circulation (EHC), and impaired liver regeneration ${ }^{9}$. Also, PTBD can be complicated by seeding metastasis, affecting patients' survival ${ }^{15}, 16$. EBD, including endoscopic biliary stenting (EBS, internal) and endoscopic nasobiliary drainage (ENBD, external) modes, is considered less invasive and avoids the potential disadvantages of PTBD. However, EBD can result in cholangitis, as it creates a direct connection between the proximal intestine and the biliary system, a risk for postoperative death 8,17 .

Several systematic reviews (SRs) ${ }^{18-28}$ addressed the effects of preoperative BD versus no preoperative $\mathrm{BD}$, as well as $\mathrm{PTBD}$ versus EBD on drainage-related and/or postoperative outcomes in patients with resectable pCCA. However, these reviews ${ }^{18-}$ 28 reported inconsistent results on the same outcomes indicators. For example, two SRs ${ }^{18,19}$ showed significantly higher overall postoperative morbidity in patients with 
preoperative BD. In contrast, two other SRs ${ }^{20,21}$ found no significant difference in overall postoperative morbidity between drained and undrained patients. Moreover, the methodological quality of published SRs is undetermined, and risk of bias may exist. These limitations of previous SRs make it difficult for clinicians to decide whether or not to perform BD and which method to employ to minimize morbidity for patients with resectable pCCA.

This review, therefore, aims to systematically study all SRs with available evidence to assess the effect of preoperative $\mathrm{BD}$ versus no preoperative $\mathrm{BD}$, and the superiority of EBD versus PTBD on perioperative and long-term outcomes in patients with resectable pCCA. Particularly, we also aimed to evaluate the risk of bias (study quality) of available SRs.

\section{Methods}

This study was reported in accordance with the Preferred Reporting Items for Systematic Reviews and Meta-Analyses (PRISMA) statement ${ }^{29}$. After a preliminary literature survey, the protocol was written and registered at the International Prospective Register of Systematic Reviews (PROSPERO) with registration number CRD42019141412

(https://www.crd.york.ac.uk/prospero/display_record.php?ID=CRD42019141412).

\section{Search strategy}

We used a combination of Medical Subject Headings (MeSH) and free-text terms to search PubMed, Embase (Ovid), Cochrane Library, and KSR Evidence (https://ksrevidence.com, it is a database that includes all systematic reviews and meta-analyses published since 2015) from inception until May 31, 2019. We also searched PROSPERO and ClinicalTrials.gov to identify any unpublished studies. The search strategy was developed in collaboration with a certified librarian and further refined by another experienced reviewer (JK) using the following conceptual groups: (1) biliary drainage, (2) perihilar cholangiocarcinoma, and (3) mortality/survival (Appendix S2.1, Supplementary file). No language restrictions were applied in any of the databases. Searches were updated on March 31, 2020. We checked the references in included papers for further studies.

\section{Study selection}


Two investigators (XC and HS) independently screened the titles and abstracts and then full texts to identify eligible studies for inclusion. Any disagreements were resolved by discussion. We selected the most recent version in the case of duplicate reports.

We included studies which met the following criteria: (1) studies included adult patients ( $\geq 18$ years old) with resectable pCCA; $(2)$ studies investigated whether or not to perform preoperative BD or the effect of EBD compared with PTBD in PCCA; (3) studies reported on at least one of these outcomes: drainage-related morbidity (e.g. cholangitis, pancreatitis, portal vein injury, cancer seeding, and bleeding), postoperative morbidity (e.g. liver failure, sepsis, and bile leakage), postoperative mortality, survival; (4) studies were SRs, with or without meta-analysis.

We excluded studies involving patients with obstructive jaundice not due to pCCA, such as autoimmune diseases (e.g. IgG4-related autoimmune cholangitis or primary sclerosing cholangitis) or bile duct stones. Also, we excluded narrative reviews (other than SRs) due to the absence of pre-specified eligibility criteria and systematic methodology, as well as SRs of SRs, commentaries, conference proceedings, and editorials.

\section{Data collection}

Two reviewers (XC and HS) independently extracted data using pre-specified data collection forms. Discrepancies were resolved through discussion. Data extraction included SR characteristics (e.g. the inclusion criteria, intervention type, numbers of included studies and participants, types of study design, quality assessment of studies, analytical approach, and conclusions) and original study characteristics included in the SRs (e.g. numbers of participants and events, and intervention type). The primary outcome was postoperative mortality. The secondary outcomes were overall drainagerelated morbidity, drainage-related cholangitis and pancreatitis, overall postoperative morbidity, postoperative major morbidity (Clavien-Dindo grade III-IV), infectious morbidity, liver failure, seeding metastasis, and long-term survival. It should be noted that for this study, the setting of the outcome indicator 'long-term survival' was modified from the registered protocol (CRD42019141412). The final literature survey revealed that long-term survival was reported in a single SR only (comparing BD with no BD), hence, it was deemed appropriate to include long-term survival as a secondary outcome measure. 


\section{Risk-of-bias assessment}

Two investigators (XC and $\mathrm{HS}$ ) independently assessed the risk of bias for each included SR using the Risk Of Bias In Systematic reviews (ROBIS) tool ${ }^{30}$, a reliable and widely used appraisal instrument 31,32 . All disagreements were solved by discussion. The ROBIS tool includes 3 phases ${ }^{30}$, of which the first phase assesses relevance (optional). The second phase covers 4 domains (21 items): study eligibility criteria, identification and selection of studies, data collection and study appraisal, and synthesis and findings. The third phase evaluates the overall risk of bias in the SRs. The items are rated as yes, probably yes, probably no, no, and no information. As PRISMA ${ }^{33}$ and ROBIS ${ }^{30}$ recommended, we assigned a rating of "low risk of bias", "high risk of bias", or "unclear risk of bias" to the overall risk of bias, instead of a summary score, because the latter may mask critical weaknesses decreasing the confidence in the results of a SR ${ }^{33,34}$.

\section{Data synthesis}

Data were evaluated using qualitative synthesis. Descriptive statistics were reported as frequency (percentage) when possible. We reported summary estimates of preoperative BD (EBD and/or PTBD) effects on primary and secondary outcomes, as relative risk $(R R)$ or odds ratio $(O R)$ with $95 \%$ confidence interval $(\mathrm{Cl})$ for dichotomous data, and hazard ratios (HR) with 95\% Cls for time-to-event data. Because many included SRs comprised data from overlapping studies, a meta-analysis could not be performed.

\section{Results}

\section{Study selection}

The database searches identified 2626 records including 5950 patients/2478 citations from the initial search (May 31, 2019) and an additional 148 abstracts from the update (March 31, 2020). After de-duplicating, screening titles and abstracts, 2586 records were excluded. Through completely reviewing the full text of the remaining 40 articles, we included 11 eligible SRs with meta-analyses. The included 11 SRs are published in English language, and no studies in a language other than English, were eligible for inclusion in the present study. No additional studies were eligible for inclusion after checking the references of included studies. Of eleven included SRs, four compared 
preoperative $\mathrm{BD}$ with no preoperative $\mathrm{BD}$, while seven compared $\mathrm{EBD}$ with $\mathrm{PTBD}$ in patients with pCCA (Fig. 1). The excluded full-text reports with reasons are presented in Supplementary Table S1.

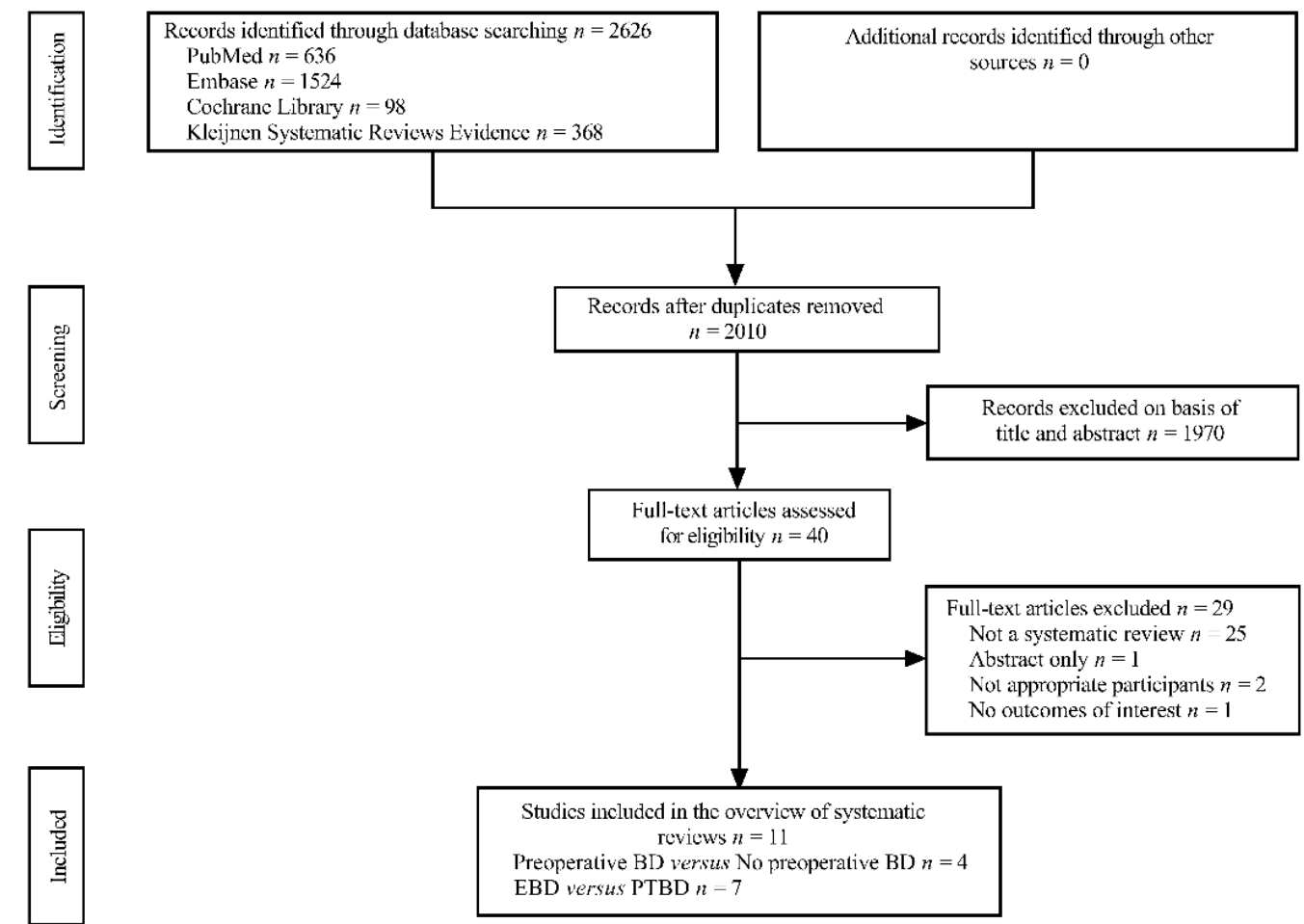

Figure 1. PRISMA flow chart showing selection of studies for review. BD, biliary drainage; EBD, endoscopic biliary drainage; PTBD, percutaneous transhepatic biliary drainage.

Comparison of effects between preoperative BD and no preoperative BD in resectable pCCA

Study characteristics

Characteristics of the four SRs ${ }^{18-21}$ evaluating the effects of preoperative BD are shown in Table 1. These SRs ${ }^{18-21}$ reported 33 unique studies, ranging from 9 to 19 studies with 711 to 2178 participants per individual SR. Eleven studies were repeatedly included in at least two SRs. Three 18, 19, 21 of four SRs only included participants who underwent resection. The other SR ${ }^{20}$ also included participants where metastatic spread prevented actual resection. All original studies included were retrospective except for a single prospective cohort study ${ }^{35}$. The quality of included original studies was low to moderate ${ }^{19-21}$. The SR by Liu et al. ${ }^{18}$ reported 252 (54.1\%) participants 
receiving external drainage, $196(42.1 \%)$ internal drainage, and $18(3.8 \%)$ receiving a combination of internal and external drainage among 466 drained participants. Two SRs reported mean preoperative total bilirubin levels of $9.6^{18}$ and $4.6 \mathrm{mg} / \mathrm{dL}^{20}$ in the drained patients, and $16.3^{18}$ and $15.8 \mathrm{mg} / \mathrm{dL}^{20}$ in the patients without drainage, respectively. The mean duration between $B D$ initiation and hepatic resection was 22.8 ${ }^{18}$ and 30.8 days ${ }^{20}$, respectively.

Risk of bias of included systematic reviews

All SRs 18-21 were rated as "at high risk of bias" using ROBIS assessment (Supplementary Table S2). The Cohen's kappa coefficient for inter-rater agreement between the two reviewers was 0.76 . One SR ${ }^{18}$ was classified as "high risk of bias" mainly due to lacking quality assessment of original studies. The high risk of bias rating in the remaining SRs ${ }^{19-21}$ was due to a number of concerns, such as language restrictions, lack of search window, not considering bias of primary studies in the synthesis, and incomplete reporting of independence in studies identification and data extraction.

Besides, the numbers of included studies and abstracted participants and events were variable across the SRs ${ }^{18-21}$ (Supplementary Table S3, Table S4), reflecting a combination of difference in period, inclusion criteria, or carelessness.

Effects of preoperative BD on primary outcome

Postoperative mortality

All SRs ${ }^{18-21}$ reported postoperative mortality in patients with pCCA (Table 2). These SRs consistently showed no statistically significant difference in postoperative mortality between patients with and without preoperative BD (OR 0.70, 95\% Cl 0.41 1.19 18; RR 0.935, 95\% Cl 0.612-1.429 19; OR 0.94, 95\% Cl 0.54-1.63 20; and OR 1.06, $\left.95 \% \mathrm{Cl} 0.70-1.61^{21}\right)$. However, these findings were derived from unadjusted ORs or $\mathrm{RR}$. The definitions of mortality were variable and ranged from postoperative day 30 to $9010,14,36-38$. 
Preoperative biliary drainage in $\mathrm{pCCA}$














Effects of preoperative BD on secondary outcomes

Overall postoperative morbidity

All SRs ${ }^{18-21}$ reported on overall postoperative morbidity (Table 2). Of these, two demonstrated that preoperative BD was associated with significantly higher overall postoperative morbidity compared to immediate surgery (OR 1.67, 95\% Cl 1.17-2.39 18 and RR $1.266,95 \% \mathrm{Cl} 1.039-1.543{ }^{19}$ ), however, the other two SRs ${ }^{20,} 21$ found no significant difference between two groups. Through subgroup analysis, Yan et al. 20 showed higher overall postoperative morbidity in the preoperative BD group in the early 5 years' studies (2006-2011) (OR 2.67, 95\% Cl 1.71-4.16), but not in the late 5 years' studies (2012-2016).

\section{Postoperative major morbidity}

Postoperative major morbidity was reported by one SR ${ }^{21}$ revealing significantly higher major morbidity in the preoperative BD group (OR 1.51, 95\% Cl 1.14-2.00) (Table 2). However, due to different indications for BD in the original studies, Mehrabi et al. ${ }^{21}$ performed a subgroup analysis. Patients were classified into a simple criteria group where preoperative BD was routinely used in all jaundiced patients, and a strict criteria group where preoperative BD was performed in patients with cholangitis and/or total bilirubin levels $\geq 15.0 \mathrm{mg} / \mathrm{dL}$ and/or inadequate future liver remnant (FLR) volume in addition to jaundice ${ }^{21}$. They found that preoperative BD was associated with increased postoperative major morbidity in 'simple criteria' patients (OR 1.57, 95\% Cl 1.10-2.25), but not in strictly selected patients (OR $0.51,95 \% \mathrm{Cl} 0.18-1.42)^{21}$.

\section{Postoperative infectious morbidity}

Of the two SRs that reported on postoperative infectious morbidity, one ${ }^{18}$ showed a significant increase of infectious morbidity in patients receiving preoperative BD (OR 2.17, 95\% Cl 1.24-3.80) (Table 2). However, the infectious morbidity was similar in the other SR ${ }^{20}$. Celotti et al. ${ }^{19}$ evaluated postoperative wound infections, showing significantly higher infection rates in the preoperative BD group (RR 2.035, 95\% Cl 1.041-3.977).

Postoperative long-term survival

Postoperative long-term survival was assessed by one SR ${ }^{20}$ and no difference between drained and undrained patients was observed (Table 2). 


\section{Comparison of effects between EBD and PTBD in resectable pCCA}

Study characteristics

Characteristics of the seven SRs ${ }^{22-28}$ investigating the effect of EBD compared to PTBD are presented in Table 3. These SRs included 24 original studies in total, ranging from 4 to 15 studies with 433 to 1230 participants per SR. Eight studies were repeatedly included in at least two SRs. Three $22,26,27$ of seven SRs only included participants who underwent resection. All original studies included were retrospective studies. The quality of included original studies was moderate to high ${ }^{24-28}$. One SR 27 reported preoperative total bilirubin levels, ranging from 5.2 to $9.6 \mathrm{mg} / \mathrm{dL}$ and 8.4 to $12.0 \mathrm{mg} / \mathrm{dL}$ in the EBD and PTBD group, respectively. One SR reported median duration between drainage and surgery of 19 and 15 days in the EBD and PTBD group, respectively ${ }^{22}$. Patients receiving PTBD had more advanced tumors compared to those receiving EBD in terms of Bismuth type IV 27,28 and American Joint Committee on Cancer classification T3/4 stage 26,28 .

Risk of bias of included systematic reviews

All but one SR ${ }^{22-27}$ were classified as "at high risk of bias" using the ROBIS tool (Supplementary Table S5). A single SR ${ }^{28}$ was rated as "at low risk of bias". The Cohen's kappa coefficient for inter-rater agreement was 0.77 . Four SRs ${ }^{24-27}$ received the rating at high risk of bias mainly because of the restrictions in publication date and language. One $\mathrm{SR}^{22}$ was assessed as high risk of bias due to the absence of quality assessment of the included studies and restrictions in publication date. The remaining $\mathrm{SR}{ }^{23}$ rated as high risk of bias was due to the restriction in language and a lack of appropriate range of search databases and sensitivity analysis.

In addition, the numbers of studies incorporated and participants and outcomes abstracted were reported inconsistently across the SRs ${ }^{22-28}$ (Supplementary Table S6, Table S7).

Comparison of effects between EBD and PTBD on primary outcomes Postoperative mortality

Four SRs 22, 23, 25, 28 compared the effect of EBD versus PTBD on postoperative mortality (Table 4). Of these, three showed no statistically significant difference between the EBD group and the PTBD group (OR 0.47, 95\% CI 0.17-1.24 23; OR 0.63, 
95\% Cl 0.19-2.10 25; and OR 0.61, 95\% Cl 0.31-1.22 ${ }^{28}$ ). The remaining SR ${ }^{22}$ reported the percentage of deaths following resection, viz. $2 \%(6 / 281)$ and $6 \%(23 / 416)$ in EBD group and PTBD group, respectively $(p=0.028)$.

Comparison of effects between EBD and PTBD on secondary outcomes

Overall drainage-related morbidity

Four SRs 22, 23, 25, 28 evaluated overall drainage-related morbidity. Three SRs demonstrated significantly higher drainage-related morbidity following an EBD procedure compared to PTBD (OR 2.23, 95\% Cl 1.39-3.57 23; OR 3.13, 95\% Cl 1.96$5.01^{25}$; and OR 2.24, 95\% Cl 1.38-3.63 ${ }^{28}$ ) (Table 4). However, this significant difference was not noted in the SR by Hameed et al. ${ }^{22}$.

\section{Drainage-related cholangitis}

Of the five SRs ${ }^{22-25,28}$ assessing drainage-related cholangitis, three pointed towards significantly higher cholangitis rates in the EBD group compared to the PTBD group (OR 5.41, 95\% Cl 2.75-10.63 23; RR 0.49, 95\% Cl 0.31-0.76 (PTBD versus EBS) ${ }^{24}$; and $\mathrm{OR} 4.58,95 \% \mathrm{Cl} 2.20-9.52{ }^{28}$ ) (Table 4). However, cholangitis rates were not affected by drainage procedure in the two other SRs ${ }^{22,25}$.

Drainage-related pancreatitis

Drainage-related pancreatitis was assessed by three SRs ${ }^{23,25,28}$. The pancreatitis rates were consistently significantly higher in the EBD group compared to the PTBD group (OR 7.46, 95\% Cl 3.02-18.44 23; OR 11.52, 95\% Cl 2.59-51.30 25; and OR 8.90, 95\% Cl 1.74-45.44 ${ }^{28}$ ) (Table 4).

Overall postoperative morbidity

Two SRs ${ }^{23,} 25$ reported overall postoperative morbidity, which was not significantly different between the two drainage procedures (Table 4).

Postoperative major morbidity

One SR ${ }^{28}$ assessed postoperative major morbidity and revealed no significant differences between EBD with PTBD (Table 4). 
Preoperative biliary drainage in $\mathrm{pCCA}$






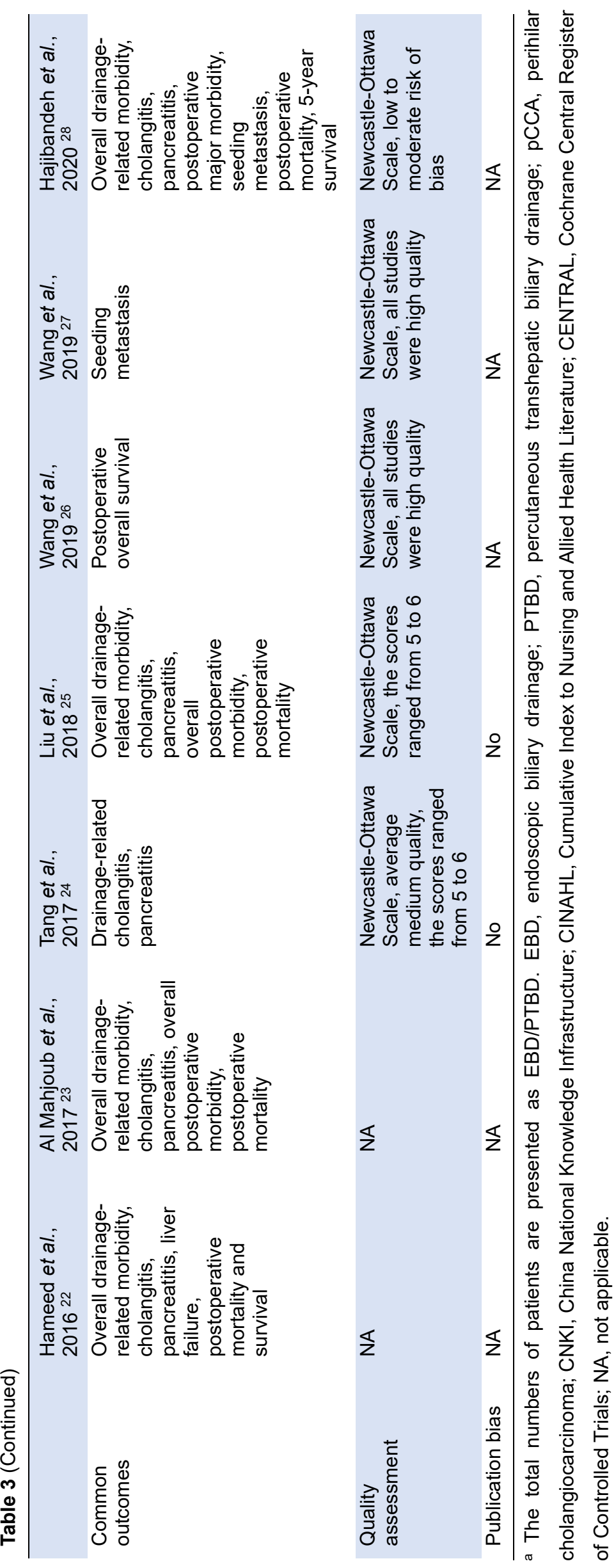


Preoperative biliary drainage in $\mathrm{pCCA}$

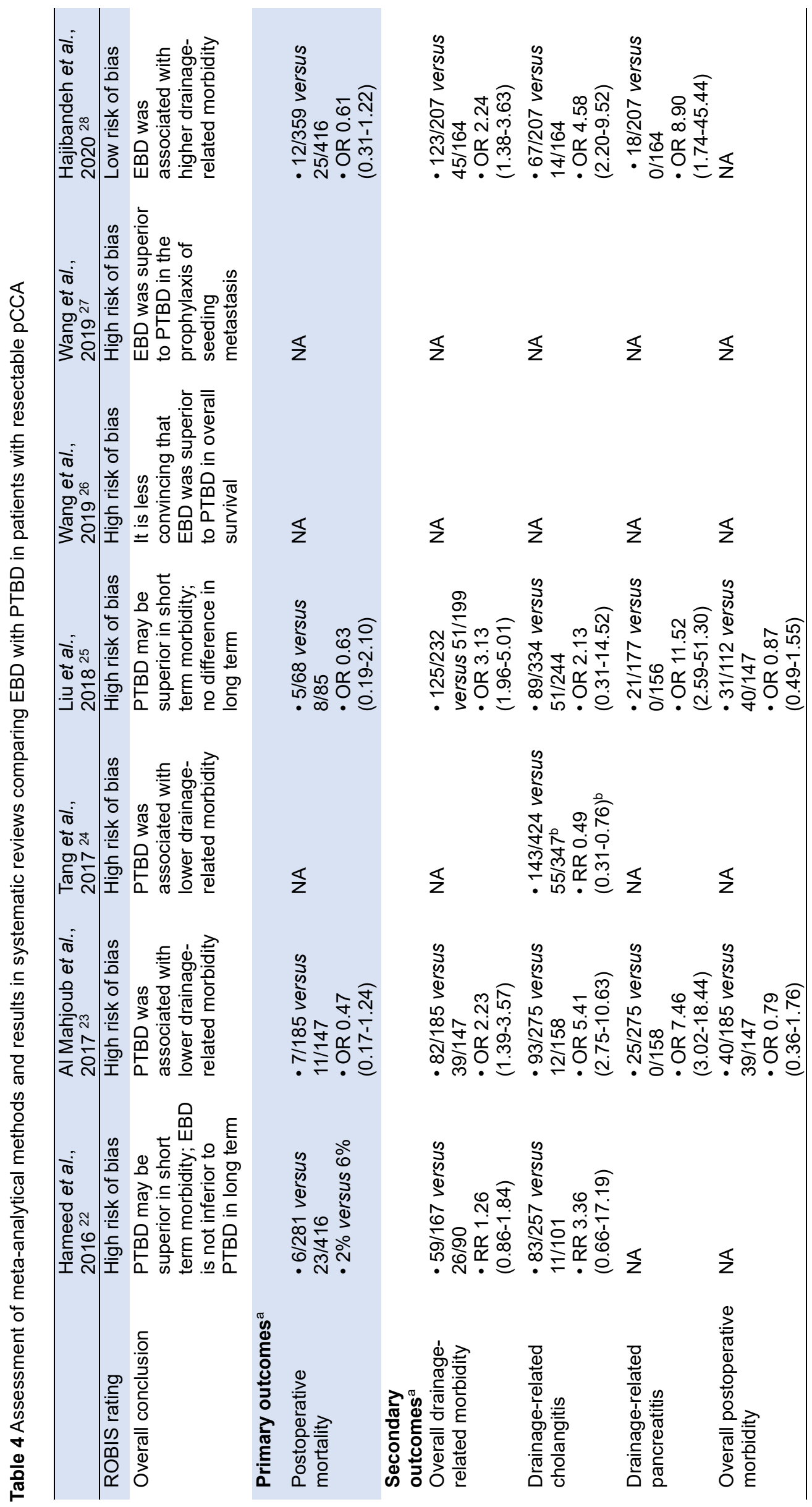




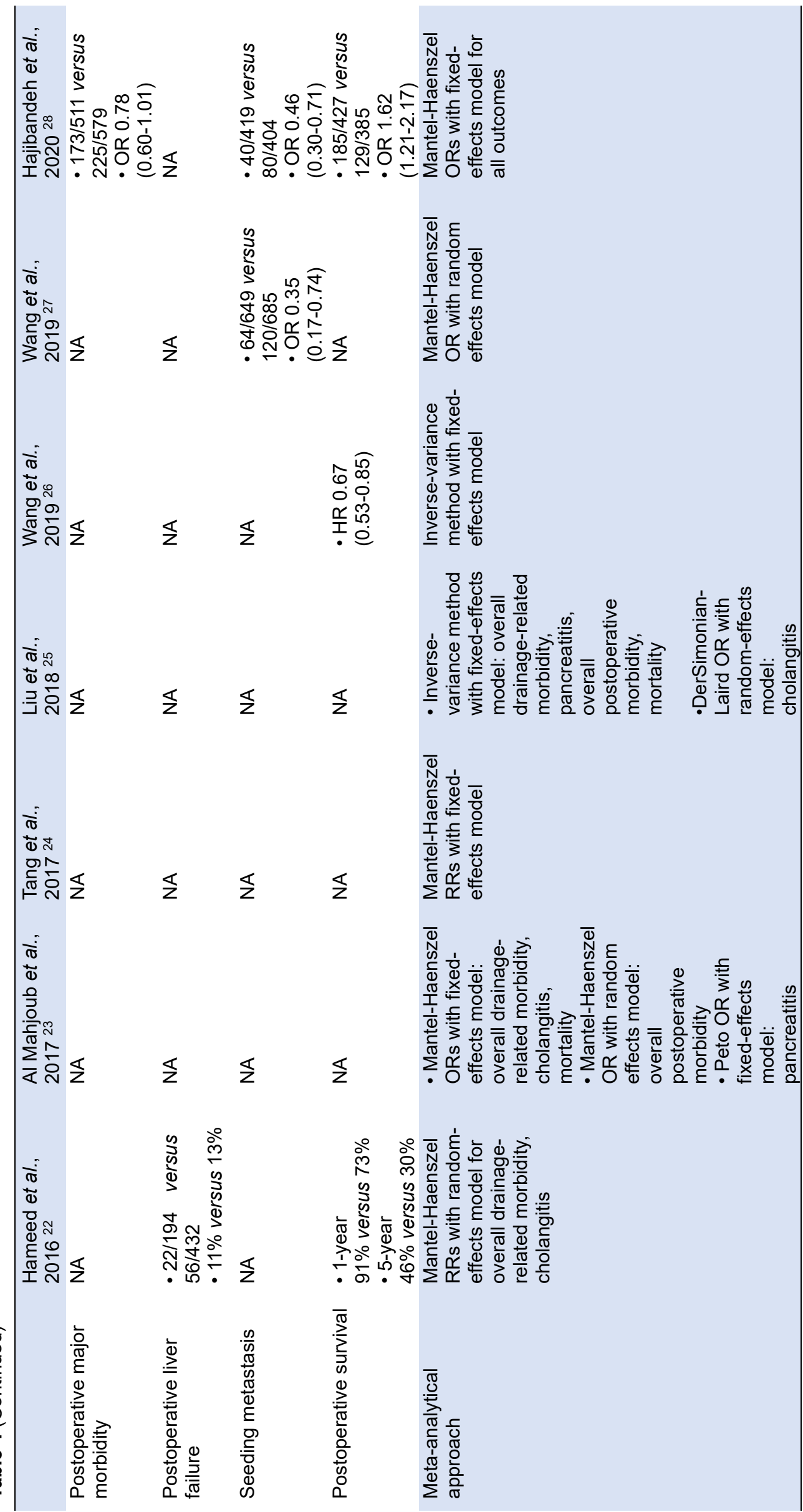


Preoperative biliary drainage in PCCA






\section{Postoperative liver failure}

Postoperative liver failure rates were evaluated in one SR ${ }^{22}$, where similar incidence rates were observed, 11\% (22/194) and 13\% (56/432) in the EBD and PTBD group, respectively $(p=0.570)$ (Table 4$)$.

\section{Seeding metastasis}

Two SRs reported the incidence of drainage-related seeding metastasis and unanimously showed lower rates in the EBD group (OR 0.27, 95\% $\mathrm{Cl} 0.13-0.56{ }^{27}$ and OR $0.46,95 \% \mathrm{Cl} \mathrm{0.30-0.7128}$ ) (Table 4).

\section{Postoperative long-term survival}

Of the three SRs $22,26,28$ assessing postoperative survival, Wang et al. ${ }^{26}$ and Hajibandeh et al. ${ }^{28}$ revealed that patients receiving EBD had longer overall survival (HR 0.67, 95\% 0.53-0.85) and 5-year survival (OR 1.62, 95\% Cl 1.21-2.17), respectively, than those receiving PTBD (Table 4). Hameed et al. ${ }^{22}$ showed that median 1 -year postoperative survival was $91 \%$ and $73 \%, 5$-year survival $46 \%$ and $30 \%$ in EBD and PTBD group, respectively.

\section{Discussion}

We performed a SR of SRs to assess the effect and route of preoperative BD in patients with resectable pCCA. The available evidence reflected conflicting results, and identified substantial variation in data abstraction and statistical methods, and high risk of bias in most included SRs. Preoperative BD may need to be used in strictly selected patients with resectable pCCA. EBD might be associated with more shortterm drainage-related morbidity compared to PTBD in patients with resectable pCCA. However, EBD might be related with better long-term outcomes after surgery.

All but one SR included in the present study are rated as high risk of bias according to ROBIS assessment. Most SRs 19, 20, 22-27 had restrictions in publication date and language. Three SRs ${ }^{18,22,23}$ did not report quality assessment of original studies. Additionally, there is considerable variability in the inclusion of studies and the numbers of participants and events abstracted across the SRs. These differences could be caused by variable period of study inclusion, inclusion criteria, and data extraction errors. The errors in data extraction procedure are a vital source of bias in SRs ${ }^{39}$. The 
influence of risk of bias on pooled results could underestimate or overestimate the actual intervention effects, resulting in a limitation of the validity of the conclusions ${ }^{40}$. The indications for preoperative BD have not reached unanimity. Elevated serum bilirubin levels are generally used as indicator for BD. A recent study demonstrated that preoperative BD should be performed when serum bilirubin $\geq 6.0 \mathrm{mg} / \mathrm{dL}$ and not recommended when bilirubin levels $<2.5 \mathrm{mg} / \mathrm{dL}^{6}$. Imaging of the liver plays an important role in the management of $\mathrm{pCCA}$ and the use of $\mathrm{BD}{ }^{41}$. FLR volume assessment by computed tomography (CT) or magnetic resonance imaging (MRI) is another vital determinant to perform BD ${ }^{42}$. For patients with FLR volumes $<50 \%$, preoperative drainage can be beneficial to decrease mortality, but not for large FLR volumes $>50 \%{ }^{42}$. Normalization of bile duct diameters in the FLR on ultrasound examination and $20 \%$ or more decrease of total bilirubin levels after 7 days, are considered indicators of therapeutic success of BD ${ }^{9}$. The SR of Mehrabi et al. ${ }^{21}$ revealed that preoperative $\mathrm{BD}$ was not associated with postoperative major morbidity in strictly selected patients with cholangitis and/or high bilirubin levels ( $\geq 15.0 \mathrm{mg} / \mathrm{dL}$ ) and/or inadequate FLR. In contrast, increased postoperative major morbidity was observed in simple criteria patients where preoperative BD was routinely used in all jaundiced patients ${ }^{21}$.

We found that postoperative mortality was similar between drained and undrained patients. This finding seems consistent with previous studies ${ }^{43}, 44$, which concluded that preoperative $\mathrm{BD}$ does not improve postoperative mortality. However, the latter conclusions were derived from unadjusted odds ratios (ORs) or relative risk (RR), without considering tumor features and resection methods. Hence, we cannot decisively conclude whether preoperative BD has effects on postoperative mortality.

EBD can be associated with high drainage-related morbidity caused by retrograde bacterial contamination from the proximal small intestine ${ }^{15,28}$. Our study demonstrated that overall drainage-related morbidity and pancreatitis rates were higher in the EBD group than in the PTBD group (ORs from 2.23 to $3.13,7.46$ to 11.52 , respectively). Hameed et al. 22 indicated no differences in overall drainage-related morbidity between the two drainage routes. However, they included fewer original articles in their $\mathrm{SR}$, which was therefore more susceptible to publication bias. Although PTBD seems to induce less drainage-related morbidity, other major complications must be considered, including tumor seeding. We found that PTBD was associated with 
significantly higher incidence of seeding metastasis, which was consistent with other studies ${ }^{16,45}$.

Our analysis showed that mortality was not statistical different in EBD versus PTBD in most included SRs. However, a recently published Dutch randomized controlled trial $(\mathrm{RCT}){ }^{9}$ demonstrated significantly higher mortality in PTBD group compared to EBD group ${ }^{9}$. Importantly, in the RCT both perioperative and postoperative mortality were assessed. PTBD significantly increased perioperative mortality, with 3 of 11 fatalities occurring before surgery in patients receiving $\mathrm{PTBD}^{9}$.

Most included SRs did not state whether internal or external drainage was used. However, internal drainage elicits a different physiological response compared to external drainage. The latter drainage mode abrogates the digestive, signaling and antimicrobial roles of bile salts in EHC. Diminished activation of the ileal bile salt receptor farnesoid $X$ receptor (FXR) can result in the loss of negative feedback control of hepatic bile salt synthesis and attendant bile salt overload and hepatotoxicity ${ }^{46}$. Importantly, bile salt signaling and maintained bile salt homeostasis is required for proper regrowth of the remnant liver ${ }^{47,48}$. Liver regeneration volumes and rates are positively associated with serum bile salts levels in patients undergoing major hepatectomy ${ }^{49}$. However, bile can be easily given back orally with external drainage mode ${ }^{50}$.

Overall, preoperative BD has merits and limitations. Preoperative BD can decompress the biliary obstruction, mitigate intrahepatic bile salt overload, relieve cholangitis, and improve liver function $8,10,11$. Moreover, the restoration of bile flow to the small intestine may improve epithelium function of gut and decrease bacterial translocation ${ }^{51}$. However, preoperative BD can result in complications. For example, PTBD is related to bile leakage and vascular complications including haemobilia and pseudoaneurysm ${ }^{52}$. In addition, tumor seeding metastasis might be another potential risk ${ }^{27}$. EBD can be associated with drainage-related cholangitis and pancreatitis, which may have nonspecific clinical manifestations ${ }^{23,28}$.

Our SR has certain strengths. First, a pre-specified protocol was followed in the review procedure, and a comprehensive and robust search strategy by two information experts was used to identify studies. Second, we used a validated instrument, ROBIS tool, to specifically assess the risk of bias in the included SRs. Finally, identification of studies, data collection, and study appraisal was performed by two reviewers 
independently to minimize potential bias.

Our SR also has several limitations, mainly caused by limitations in the included studies. First, eight 19, 20, 22-27 of eleven SRs had restrictions in publication date and/or English language. All but one SR did not include unpublished or grey literature in their selection criteria ${ }^{18-27}$. This could narrow the breadth of data sources and exclude potentially eligible studies. Second, because many original studies were repeatedly included in at least two SRs and there was heterogeneity in the definitions of outcomes, we could not calculate overall pooled estimates. Third, the ORs and/or RRs of primary and secondary outcomes were only reported in only few SRs, therefore, the results need to be interpreted with caution. Finally, all but one of the original studies included in the SRs ${ }^{18-28}$ had a retrospective design, resulting in selection bias.

It is unclear from the SRs ${ }^{22-28}$ if in PTBD procedures bile was given back to the patients (to restore functionality of the EHC). In the Dutch RCT, external drainage of bile may be a reason for the increased mortality in patients receiving PTBD ${ }^{9}$. Pre-clinical studies have demonstrated FXR-mediated acceleration of liver growth in absence ${ }^{53}$ and presence ${ }^{48}$ of partial hepatectomy. Recent clinical trials have also shown that FXR activation can improve cholestasis in patients with primary biliary cirrhosis ${ }^{54}$ and primary sclerosing cholangitis ${ }^{55}$, and liver histological features in patients with primary biliary cholangitis ${ }^{56}$. Bile salt supplementation or FXR agonism may have the potential to replace preoperative $\mathrm{BD}$ to improve perioperative outcomes in patients with pCCA, considering the activation of pathways involved in bile salt homeostasis and liver regeneration ${ }^{46-48}$.

\section{Conclusion}

The present SR of SRs comprehensively assesses the effectiveness of PBD versus no PBD, and the superiority of EBD versus and PTBD on perioperative and long-term outcomes in patients with resectable pCCA. The preoperative BD might need to be performed in strictly selected patients in terms of cholangitis, bilirubin levels and future liver remnant volume to avoid increased postoperative major morbidity. EBD might be associated with higher short-term drainage-related morbidity compared to PTBD. But EBD might be related with more favorable postoperative long-term outcomes. The available evidence has high risk of bias. Large sample sizes and/or international 
Chapter 2

multicentre RCTs are urgently needed to assess the value of preoperative BD in surgical management of patients with resectable pCCA. 


\section{References}

1. Razumilava N, Gores GJ. Cholangiocarcinoma. The Lancet. 2014;383:2168-79.

2. Khan SA, Tavolari S, Brandi G. Cholangiocarcinoma: Epidemiology and risk factors. Liver Int. 2019;39 Suppl 1:19-31.

3. Blechacz B, Komuta M, Roskams T, Gores GJ. Clinical diagnosis and staging of cholangiocarcinoma. Nat Rev Gastroenterol Hepatol. 2011;8:512-22.

4. Mizuno T, Ebata T, Yokoyama Y, Igami T, Yamaguchi J, Onoe S, et al. Combined Vascular Resection for Locally Advanced Perihilar Cholangiocarcinoma. Ann Surg. 2020.

5. Nagino M, Ebata T, Yokoyama Y, Igami T, Sugawara G, Takahashi Y, et al. Evolution of surgical treatment for perihilar cholangiocarcinoma: a single-center 34-year review of 574 consecutive resections. Ann Surg. 2013;258:129-40.

6. Wronka KM, Grat M, Stypulkowski J, Bik E, Patkowski W, Krawczyk M, et al. Relevance of Preoperative Hyperbilirubinemia in Patients Undergoing Hepatobiliary Resection for Hilar Cholangiocarcinoma. J Clin Med. 2019;8:458.

7. Inamdar S, Slattery E, Bhalla R, Sejpal DV, Trindade AJ. Comparison of Adverse Events for Endoscopic vs Percutaneous Biliary Drainage in the Treatment of Malignant Biliary Tract Obstruction in an Inpatient National Cohort. JAMA Oncol. 2016;2:112-7.

8. Lidsky ME, Jarnagin WR. Surgical management of hilar cholangiocarcinoma at Memorial Sloan Kettering Cancer Center. Ann Gastroenterol Surg. 2018;2:304-12.

9. Coelen RJS, Roos E, Wiggers JK, Besselink MG, Buis Cl, Busch ORC, et al. Endoscopic versus percutaneous biliary drainage in patients with resectable perihilar cholangiocarcinoma: a multicentre, randomised controlled trial. Lancet Gastroenterol Hepatol. 2018;3:681-90.

10. Farges O, Regimbeau JM, Fuks D, Le Treut YP, Cherqui D, Bachellier P, et al. Multicentre European study of preoperative biliary drainage for hilar cholangiocarcinoma. $\mathrm{Br} \mathrm{J}$ Surg. 2013;100:274-83.

11. Reiniers MJ, de Haan L, Weijer R, Wiggers JK, Jongejan A, Moerland PD, et al. Effect of preoperative biliary drainage on cholestasis-associated inflammatory and fibrotic gene signatures in perihilar cholangiocarcinoma. Br J Surg. 2019;106:55-8.

12. Ramanathan R, Borrebach J, Tohme S, Tsung A. Preoperative Biliary Drainage Is Associated with Increased Complications After Liver Resection for Proximal Cholangiocarcinoma. J Gastrointest Surg. 2018;22:1950-7.

13. Ratti F, Cipriani F, Fiorentini G, Hidalgo Salinas C, Catena M, Paganelli M, et al. Management of hilum infiltrating tumors of the liver: The impact of experience and standardization on outcome. Dig Liver Dis. 2019;51:135-41.

14. Xiong JJ, Nunes QM, Huang W, Pathak S, Wei AL, Tan CL, et al. Preoperative biliary drainage in patients with hilar cholangiocarcinoma undergoing major hepatectomy. World J Gastroenterol. 2013;19:8731-9.

15. Kim KM, Park JW, Lee JK, Lee KH, Lee KT, Shim SG. A Comparison of Preoperative Biliary Drainage Methods for Perihilar Cholangiocarcinoma: Endoscopic versus Percutaneous Transhepatic Biliary Drainage. Gut Liver. 2015;9:791-9.

16. Komaya K, Ebata T, Yokoyama Y, Igami T, Sugawara G, Mizuno T, et al. Verification of the oncologic inferiority of percutaneous biliary drainage to endoscopic drainage: A propensity score matching analysis of resectable perihilar cholangiocarcinoma. Surgery. 2017;161:394-404.

17. Ribero D, Zimmitti G, Aloia TA, Shindoh J, Fabio F, Amisano M, et al. Preoperative Cholangitis 
and Future Liver Remnant Volume Determine the Risk of Liver Failure in Patients Undergoing Resection for Hilar Cholangiocarcinoma. J Am Coll Surg. 2016;223:87-97.

18. Liu F, Li Y, Wei Y, Li B. Preoperative biliary drainage before resection for hilar cholangiocarcinoma: whether or not? A systematic review. Dig Dis Sci. 2011;56:663-72.

19. Celotti A, Solaini L, Montori G, Coccolini F, Tognali D, Baiocchi G. Preoperative biliary drainage in hilar cholangiocarcinoma: Systematic review and meta-analysis. Eur $\mathrm{J}$ Surg Oncol. 2017;43:1628-35.

20. Yan K, Tian J, Xu J, Fu Y, Zhang H, Song J. The value of preoperative biliary drainage in hilar cholangiocarcinoma: A systematic review and meta analysis of 10 years' literatures. Int $\mathrm{J}$ Clin Exp Med. 2018;11:3462-72.

21. Mehrabi A, Khajeh E, Ghamarnejad O, Nikdad M, Chang DH, Buchler MW, et al. Meta-analysis of the efficacy of preoperative biliary drainage in patients undergoing liver resection for perihilar cholangiocarcinoma. Eur J Radiol. 2020;125:108897.

22. Hameed A, Pang T, Chiou J, Pleass H, Lam V, Hollands M, et al. Percutaneous vs. endoscopic pre-operative biliary drainage in hilar cholangiocarcinoma - a systematic review and metaanalysis. HPB 2016;18:400-10.

23. Al Mahjoub A, Menahem B, Fohlen A, Dupont B, Alves A, Launoy G, et al. Preoperative Biliary Drainage in Patients with Resectable Perihilar Cholangiocarcinoma: Is Percutaneous Transhepatic Biliary Drainage Safer and More Effective than Endoscopic Biliary Drainage? A Meta-Analysis. J Vasc Interv Radiol. 2017;28:576-82.

24. Tang Z, Yang Y, Meng W, Li X. Best option for preoperative biliary drainage in Klatskin tumor: A systematic review and meta-analysis. Medicine 2017;96:e8372.

25. Liu JG, Wu J, Wang J, Shu GM, Wang YJ, Lou C, et al. Endoscopic Biliary Drainage Versus Percutaneous Transhepatic Biliary Drainage in Patients with Resectable Hilar Cholangiocarcinoma: A Systematic Review and Meta-Analysis. J Laparoendosc Adv Surg Tech A. 2018;28:1053-60.

26. Wang L, Lin N, Xin F, Zeng Y, Liu J. Comparison of long-term efficacy between endoscopic and percutaneous biliary drainage for resectable extrahepatic cholangiocarcinoma with biliary obstruction: A systematic review and meta-analysis. Saudi J Gastroenterol. 2019;25:81-8.

27. Wang L, Lin N, Xin F, Ke Q, Zeng Y, Liu J. A systematic review of the comparison of the incidence of seeding metastasis between endoscopic biliary drainage and percutaneous transhepatic biliary drainage for resectable malignant biliary obstruction. World J Surg Oncol. 2019;17:116.

28. Hajibandeh S, Hajibandeh S, Satyadas T. Endoscopic Versus Percutaneous Preoperative Biliary Drainage in Patients With Klatskin Tumor Undergoing Curative Surgery: A Systematic Review and Meta-Analysis of Short-Term and Long-Term Outcomes. Surg Innov. 2020;27:279-90.

29. Moher D, Liberati A, Tetzlaff J, Altman DG. Preferred reporting items for systematic reviews and meta-analyses: the PRISMA statement. BMJ. 2009;339:b2535.

30. Whiting P, Savovic J, Higgins JP, Caldwell DM, Reeves BC, Shea B, et al. ROBIS: A new tool to assess risk of bias in systematic reviews was developed. J Clin Epidemiol. 2016;69:225-34.

31. Ingoe HM, Coleman E, Eardley W, Rangan A, Hewitt C, McDaid C. Systematic review of systematic reviews for effectiveness of internal fixation for flail chest and rib fractures in adults. BMJ Open. 2019;9:e023444.

32. Siegfried N, Parry C. Do alcohol control policies work? An umbrella review and quality assessment of systematic reviews of alcohol control interventions (2006 - 2017). PLoS One. 
2019;14:e0214865.

33. Beller EM, Glasziou PP, Altman DG, Hopewell S, Bastian H, Chalmers I, et al. PRISMA for Abstracts: reporting systematic reviews in journal and conference abstracts. PLoS Med. 2013;10:e1001419.

34. Shea BJ, Reeves BC, Wells G, Thuku M, Hamel C, Moran J, et al. AMSTAR 2: a critical appraisal tool for systematic reviews that include randomised or non-randomised studies of healthcare interventions, or both. BMJ. 2017;358:j4008.

35. Regimbeau JM, Fuks D, Le Treut YP, Bachellier P, Belghiti J, Boudjema K, et al. Surgery for hilar cholangiocarcinoma: a multi-institutional update on practice and outcome by the AFC-HC study group. J Gastrointest Surg. 2011;15:480-8.

36. Ferrero A, Lo Tesoriere R, Vigano L, Caggiano L, Sgotto E, Capussotti L. Preoperative biliary drainage increases infectious complications after hepatectomy for proximal bile duct tumor obstruction. World J Surg. 2009;33:318-25.

37. El-Hanafy E. Pre-operative biliary drainage in hilar cholangiocarcinoma, benefits and risks, single center experience. Hepatogastroenterology. 2010;57:414-9.

38. Grandadam S, Compagnon P, Arnaud A, Olivie D, Malledant Y, Meunier B, et al. Role of preoperative optimization of the liver for resection in patients with hilar cholangiocarcinoma type III. Ann Surg Oncol. 2010;17:3155-61.

39. Onasanya O, lyer G, Lucas E, Lin D, Singh S, Alexander GC. Association between exogenous testosterone and cardiovascular events: an overview of systematic reviews. The Lancet Diabetes \& Endocrinology. 2016;4:943-56.

40. Higgins JP, Altman DG, Gotzsche PC, Juni P, Moher D, Oxman AD, et al. The Cochrane Collaboration's tool for assessing risk of bias in randomised trials. BMJ. 2011;343:d5928.

41. Abdel Razek AAK, El-Serougy LG, Saleh GA, Shabana W, Abd El-Wahab R. Liver Imaging Reporting and Data System Version 2018: What Radiologists Need to Know. J Comput Assist Tomogr. 2020;44:168-77.

42. Wiggers JK, Groot Koerkamp B, Cieslak KP, Doussot A, van Klaveren D, Allen PJ, et al. Postoperative Mortality after Liver Resection for Perihilar Cholangiocarcinoma: Development of a Risk Score and Importance of Biliary Drainage of the Future Liver Remnant. J Am Coll Surg 2016;223:321-31.

43. Zhang XF, Beal EW, Merath K, Ethun CG, Salem A, Weber SM, et al. Oncologic effects of preoperative biliary drainage in resectable hilar cholangiocarcinoma: Percutaneous biliary drainage has no adverse effects on survival. J Surg Oncol. 2018;117:1267-77.

44. Cai $\mathrm{Y}$, Tang $\mathrm{Q}$, Xiong X, Li F, Ye H, Song P, et al. Preoperative biliary drainage versus direct surgery for perihilar cholangiocarcinoma: A retrospective study at a single center. Biosci Trends. 2017;11:319-25.

45. Hirano S, Tanaka E, Tsuchikawa T, Matsumoto J, Kawakami H, Nakamura T, et al. Oncological benefit of preoperative endoscopic biliary drainage in patients with hilar cholangiocarcinoma. $\mathrm{J}$ Hepatobiliary Pancreat Sci. 2014;21:533-40.

46. Schaap FG, Trauner M, Jansen PL. Bile acid receptors as targets for drug development. Nat Rev Gastroenterol Hepatol. 2014;11:55-67.

47. Uriarte I, Fernandez-Barrena MG, Monte MJ, Latasa MU, Chang HC, Carotti S, et al. Identification of fibroblast growth factor 15 as a novel mediator of liver regeneration and its application in the prevention of post-resection liver failure in mice. Gut. 2013;62:899-910. 


\section{Chapter 2}

48. Huang W, Ma K, Zhang J, Qatanani M, Cuvillier J, Liu J, et al. Nuclear receptor-dependent bile acid signaling is required for normal liver regeneration. Science. 2006;312:233-6.

49. Otao R, Beppu T, Isiko T, Mima K, Okabe H, Hayashi $\mathrm{H}$, et al. External biliary drainage and liver regeneration after major hepatectomy. Br J Surg. 2012;99:1569-74.

50. Yoshida Y, Ajiki T, Ueno K, Shinozaki K, Murakami S, Okazaki T, et al. Preoperative bile replacement improves immune function for jaundiced patients treated with external biliary drainage. J Gastrointest Surg. 2014;18:2095-104.

51. Sorrentino G, Perino A, Yildiz E, El Alam G, Sleiman MB, Gioiello A, et al. Bile Acids Signal via TGR5 to Activate Intestinal Stem Cells and Epithelial Regeneration. Gastroenterology. 2020.

52. Venkatanarasimha N, Damodharan K, Gogna A, Leong S, Too CW, Patel A, et al. Diagnosis and Management of Complications from Percutaneous Biliary Tract Interventions. Radiographics. 2017;37:665-80.

53. Olthof PB, Huisman F, Schaap FG, van Lienden KP, Bennink RJ, van Golen RF, et al. Effect of obeticholic acid on liver regeneration following portal vein embolization in an experimental model. Br J Surg. 2017;104:590-9.

54. Hirschfield GM, Mason A, Luketic V, Lindor K, Gordon SC, Mayo M, et al. Efficacy of obeticholic acid in patients with primary biliary cirrhosis and inadequate response to ursodeoxycholic acid. Gastroenterology. 2015;148:751-61

55. Kowdley KV, Vuppalanchi R, Levy C, Floreani A, Andreone P, LaRusso NF, et al. A randomized, placebo-controlled, phase II study of obeticholic acid for primary sclerosing cholangitis. J Hepatol. 2020;73:94-101.

56. Bowlus CL, Pockros PJ, Kremer AE, Pares A, Forman LM, Drenth JPH, et al. Long-Term Obeticholic Acid Therapy Improves Histological Endpoints in Patients With Primary Biliary Cholangitis. Clin Gastroenterol Hepatol. 2020;18:1170-8. 


\section{Supplementary file}

Appendix S1 Search strategy used for electronic databases.

\section{PubMed}


cholangiopancreatographies[ti/ab]) OR endoscopic retrograde cholangiography[ti/ab]) OR endoscopic cholangiography[ti/ab]) OR endoscopic cholangiopancreatography[ti/ab]) OR endoscopic pancreatocholangiography[ti/ab]) OR ERCP[ti/ab]

\#20 \#16 OR \#17 OR \#18 OR \#19

\#21 ((morbidity[MeSH Terms]) OR mortality[MeSH Terms]) OR survival[MeSH Terms]

\#22 ((((morbidity[ti/ab]) OR complicat*[ti/ab]) OR mortality[ti/ab]) OR death rate[ti/ab]) OR survival[ti/ab]

\#23 \#21 OR \#22

\#24 \#15 AND \#20 AND \#23

\section{Embase}

\#1 Klatskin tumor/

\#2 ('Klatskin tumor' or 'Klatskin tumors' or 'Klatskin's tumor' or 'Klatskin's tumors').ab,ti.

\#3 exp bile duct tumor/

\#4 bile duct'.ab,ti. 


\section{Chapter 2}

\#5 (tumor or tumors or tumour or tumours or cancer or cancers or carci* or neoplas*).ab,ti.

\#6 \#4 and \#5

\#7 cholangiocarcinoma*.ab,ti.

\#8 \#3 or \#6 or \#7

\#9 (hilar or perihilar or proximal).ab,ti.

\#10 \#8 and \#9

\#11 hepatic duct'.ab,ti.

\#12 \#5 and \#11

\#13 \#1 or \#2 or \#10 or \#12

\#14 exp biliary tract drainage/

\#15 endoscopic retrograde cholangiopancreatography/

\#16 ('bile drainage' or 'bile duct drainage' or 'bile tract drainage' or 'biliary drainage' or 'nasobiliary drainage' or 'biliary decompression' or 'biliary stent' or 'biliary stenting').ab,ti.

\#17 ('endoscopic retrograde cholangiopancreatography' or 'endoscopic retrograde cholangiopancreatographies' or 'endoscopic retrograde cholangiography' or 'endoscopic cholangiography' or 'endoscopic cholangiopancreatography' or 'endoscopic pancreatocholangiography' or ERCP).ab,ti.

\#18 \#14 or \#15 or \#16 or \#17

\#19 exp morbidity/

\#20 exp complication/

\#21 exp mortality/

\#22 exp survival/

\#23 (morbidity or complicat ${ }^{\star}$ or mortality or 'death rate' or survival).ab,ti.

\#24 \#19 or \#20 or \#21 or \#22 or \#23

\#25 \#13 and \#18 and \#24

\section{Cochrane Library}

\#1 MeSH descriptor: [Klatskin Tumor] explode all trees

\#2 ('Klatskin tumor"' or 'Klatskin's tumor'):ti,ab,kw

\#3 MeSH descriptor: [Bile Duct Neoplasms] explode all trees

\#4 MeSH descriptor: [Cholangiocarcinoma] explode all trees

\#5 (bile duct):ti,ab,kw

\#6 (tumor* or tumour* or carci* or cancer* or neoplas* ${ }^{*}$ ):ti,ab,kw

\#7 \#5 and \#6

\#8 (cholangiocarcinoma*):ti,ab,kw

\#9 \#3 or \#4 or \#7 or \#8

\#10 (hilar or perihilar or proximal):ti,ab,kw

\#11 \#9 and \#10

\#12 (hepatic duct):ti,ab,kw 
Preoperative biliary drainage in $\mathrm{PCCA}$

\#13 \#6 and \#12

\#14 (bile duct bifurcation carcinoma*):ti,ab,kw

\#15 \#1 or \#2 or \#11 or \#13 or \#14

\#16 MeSH descriptor: [Cholangiopancreatography, Endoscopic Retrograde] explode all trees

\#17 ('bile drain*' or 'bile duct drain*' or 'bile tract drain*' or 'biliary drain*' or 'nasobiliary drain*'):ti,ab,kw

\#18 ('biliary decompression' or 'biliary stent'):ti,ab,kw

\#19 ('endoscopic retrograde cholangiopancreatography' or 'endoscopic retrograde cholangiopancreatographies' or 'endoscopic retrograde cholangiography' or 'endoscopic cholangiography' or 'endoscopic cholangiopancreatography' or 'endoscopic pancreatocholangiography' or ERCP):ti,ab,kw

\#20 \#16 or \#17 or \#18 or \#19

\#21 MeSH descriptor: [Morbidity] explode all trees

\#22 MeSH descriptor: [Mortality] explode all trees

\#23 MeSH descriptor: [Survival] explode all trees

\#24 (morbidity or complicat* ${ }^{*}$ or mortality OR death rate OR survival):ti,ab,kw

\#25 \#21 or \#22 or \#23 or \#24

\#26 \#15 and \#20 and \#25 
Table S1 List of excluded full text reviewed studies with reasons.

\begin{tabular}{|c|c|c|}
\hline Authors, Year & Title & $\begin{array}{l}\text { Exclusion } \\
\text { reason }\end{array}$ \\
\hline $\begin{array}{l}\text { Takada et al., } \\
2001^{1}\end{array}$ & $\begin{array}{l}\text { Is preoperative biliary drainage necessary according to } \\
\text { evidence-based medicine? }\end{array}$ & $\begin{array}{l}\text { Not a systematic } \\
\text { review }\end{array}$ \\
\hline $\begin{array}{l}\text { Belghiti et al., } \\
2005^{2}\end{array}$ & $\begin{array}{l}\text { Preoperative optimization of the liver for resection in patients } \\
\text { with hilar cholangiocarcinoma }\end{array}$ & $\begin{array}{l}\text { Not a systematic } \\
\text { review }\end{array}$ \\
\hline $\begin{array}{l}\text { Sakata et al., } \\
2005^{3}\end{array}$ & $\begin{array}{l}\text { Catheter tract implantation metastases associated with } \\
\text { percutaneous biliary drainage for extrahepatic } \\
\text { cholangiocarcinoma }\end{array}$ & $\begin{array}{l}\text { Not a systematic } \\
\text { review }\end{array}$ \\
\hline $\begin{array}{l}\text { Briggs et al., } \\
2007^{4}\end{array}$ & Investigation and management of obstructive jaundice & $\begin{array}{l}\text { Not a systematic } \\
\text { review }\end{array}$ \\
\hline $\begin{array}{l}\text { Maguchi et al., } \\
2007^{5}\end{array}$ & Preoperative biliary drainage for hilar cholangiocarcinoma & $\begin{array}{l}\text { Not a systematic } \\
\text { review }\end{array}$ \\
\hline $\begin{array}{l}\text { Nagino et al., } \\
2008^{6}\end{array}$ & $\begin{array}{l}\text { Preoperative biliary drainage for biliary tract and ampullary } \\
\text { carcinomas }\end{array}$ & $\begin{array}{l}\text { Not a systematic } \\
\text { review }\end{array}$ \\
\hline $\begin{array}{l}\text { Nimura et al., } \\
2008^{7}\end{array}$ & $\begin{array}{l}\text { Preoperative biliary drainage before resection for } \\
\text { cholangiocarcinoma (Pro) }\end{array}$ & $\begin{array}{l}\text { Not a systematic } \\
\text { review }\end{array}$ \\
\hline $\begin{array}{l}\text { van Delden et } \\
\text { al., } 2008^{8}\end{array}$ & $\begin{array}{l}\text { Percutaneous drainage and stenting for palliation of malignant } \\
\text { bile duct obstruction }\end{array}$ & $\begin{array}{l}\text { Not a systematic } \\
\text { review }\end{array}$ \\
\hline $\begin{array}{l}\text { Kawakami et } \\
\text { al., } 2011^{9}\end{array}$ & $\begin{array}{l}\text { Preoperative biliary drainage for hilar cholangiocarcinoma: } \\
\text { which stent should be selected? }\end{array}$ & $\begin{array}{l}\text { Not a systematic } \\
\text { review }\end{array}$ \\
\hline $\begin{array}{l}\text { Parodi et al., } \\
2012^{10}\end{array}$ & Endoscopic management of hilar cholangiocarcinoma & $\begin{array}{l}\text { Not a systematic } \\
\text { review }\end{array}$ \\
\hline $\begin{array}{l}\text { lacono et al., } \\
2013^{11}\end{array}$ & $\begin{array}{l}\text { Role of preoperative biliary drainage in jaundiced patients who } \\
\text { are candidates for pancreatoduodenectomy or hepatic } \\
\text { resection: highlights and drawbacks }\end{array}$ & $\begin{array}{l}\text { Not a systematic } \\
\text { review }\end{array}$ \\
\hline $\begin{array}{l}\text { Webb et al., } \\
2013^{12}\end{array}$ & Endoscopic Management of Malignant Bile Duct Strictures & $\begin{array}{l}\text { Not a systematic } \\
\text { review }\end{array}$ \\
\hline $\begin{array}{l}\text { Yasuda et al., } \\
2013^{13}\end{array}$ & $\begin{array}{l}\text { Unilateral versus bilateral endoscopic biliary stenting for } \\
\text { malignant hilar biliary strictures }\end{array}$ & $\begin{array}{l}\text { Not a systematic } \\
\text { review }\end{array}$ \\
\hline $\begin{array}{l}\text { Zhimin et al., } \\
2013^{14}\end{array}$ & $\begin{array}{l}\text { Advances in diagnosis and treatment of hilar } \\
\text { cholangiocarcinoma - A review }\end{array}$ & $\begin{array}{l}\text { Not a systematic } \\
\text { review }\end{array}$ \\
\hline $\begin{array}{l}\text { Fang et al., } \\
2013^{15}\end{array}$ & $\begin{array}{l}\text { Meta-analysis of randomized clinical trials on safety and } \\
\text { efficacy of biliary drainage before surgery for obstructive } \\
\text { jaundice }\end{array}$ & $\begin{array}{l}\text { Not appropriate } \\
\text { participants }\end{array}$ \\
\hline $\begin{array}{l}\text { Leng et al., } \\
2014^{16}\end{array}$ & $\begin{array}{l}\text { Percutaneous transhepatic and endoscopic biliary drainage for } \\
\text { malignant biliary tract obstruction: a meta-analysis }\end{array}$ & $\begin{array}{l}\text { Not appropriate } \\
\text { participants }\end{array}$ \\
\hline $\begin{array}{l}\text { Paik et al., } \\
2014^{17}\end{array}$ & $\begin{array}{l}\text { Preoperative biliary drainage in hilar cholangiocarcinoma: } \\
\text { When and how? }\end{array}$ & $\begin{array}{l}\text { Not a systematic } \\
\text { review }\end{array}$ \\
\hline $\begin{array}{l}\text { Soares et al., } \\
2014^{18}\end{array}$ & $\begin{array}{l}\text { Hilar cholangiocarcinoma: diagnosis, treatment options, and } \\
\text { management }\end{array}$ & $\begin{array}{l}\text { Not a systematic } \\
\text { review }\end{array}$ \\
\hline $\begin{array}{l}\text { Park et al., } \\
2015^{19}\end{array}$ & $\begin{array}{l}\text { Endoscopic ultrasound-guided biliary drainage of hilar biliary } \\
\text { obstruction }\end{array}$ & $\begin{array}{l}\text { Not a systematic } \\
\text { review }\end{array}$ \\
\hline $\begin{array}{l}\text { Poruk et al., } \\
2015^{20}\end{array}$ & Perioperative Management of Hilar Cholangiocarcinoma & $\begin{array}{l}\text { Not a systematic } \\
\text { review }\end{array}$ \\
\hline $\begin{array}{l}\text { Rodarte- } \\
\text { Shade et al., } \\
2015^{21}\end{array}$ & $\begin{array}{l}\text { Stent placement as a bridge to surgery in malignant biliary } \\
\text { obstruction (pancreatic cancer, distal bile duct cancer, and hilar } \\
\text { tumors) }\end{array}$ & $\begin{array}{l}\text { Not a systematic } \\
\text { review }\end{array}$ \\
\hline $\begin{array}{l}\text { Rustagi et al., } \\
2015^{22}\end{array}$ & Endoscopic treatment of malignant biliary strictures & $\begin{array}{l}\text { Not a systematic } \\
\text { review }\end{array}$ \\
\hline $\begin{array}{l}\text { Saxena et al., } \\
2015^{23}\end{array}$ & Preoperative biliary drainage & $\begin{array}{l}\text { Not a systematic } \\
\text { review }\end{array}$ \\
\hline $\begin{array}{l}\text { Tsuchikawa et } \\
\text { al., } 2015^{24}\end{array}$ & Advances in the surgical treatment of hilar cholangiocarcinoma & $\begin{array}{l}\text { Not a systematic } \\
\text { review }\end{array}$ \\
\hline
\end{tabular}


Preoperative biliary drainage in $\mathrm{pCCA}$

Table S1 (continued)

\begin{tabular}{|l|l|l|}
\hline Authors, Year & Title & $\begin{array}{l}\text { Exclusion } \\
\text { reason }\end{array}$ \\
\hline $\begin{array}{l}\text { Umeda et al., } \\
2015^{25}\end{array}$ & Current status of preoperative biliary drainage & $\begin{array}{l}\text { Not a systematic } \\
\text { review }\end{array}$ \\
\hline $\begin{array}{l}\text { Jo et al., } \\
2017^{26}\end{array}$ & $\begin{array}{l}\text { Best options for preoperative biliary drainage in patients with } \\
\text { Klatskin tumors }\end{array}$ & $\begin{array}{l}\text { Not a systematic } \\
\text { review }\end{array}$ \\
\hline $\begin{array}{l}\text { Tringali et al., } \\
2017^{27}\end{array}$ & $\begin{array}{l}\text { Endoscopic vs percutaneous preoperative biliary drainage in } \\
\text { hilar cholangiocarcinoma: A systematic review and meta- } \\
\text { analysis }\end{array}$ & Abstract only \\
\hline $\begin{array}{l}\text { Tang et al., } \\
2018^{28}\end{array}$ & $\begin{array}{l}\text { The clinicopathological factors associated with prognosis of } \\
\text { patients with resectable perihilar cholangiocarcinoma: A } \\
\text { systematic review and meta-analysis }\end{array}$ & $\begin{array}{l}\text { No outcomes of } \\
\text { interest }\end{array}$ \\
\hline $\begin{array}{l}\text { Lee et al., } \\
2020^{29}\end{array}$ & Biliary stenting for hilar malignant biliary obstruction & $\begin{array}{l}\text { Not a systematic } \\
\text { review }\end{array}$ \\
\hline
\end{tabular}




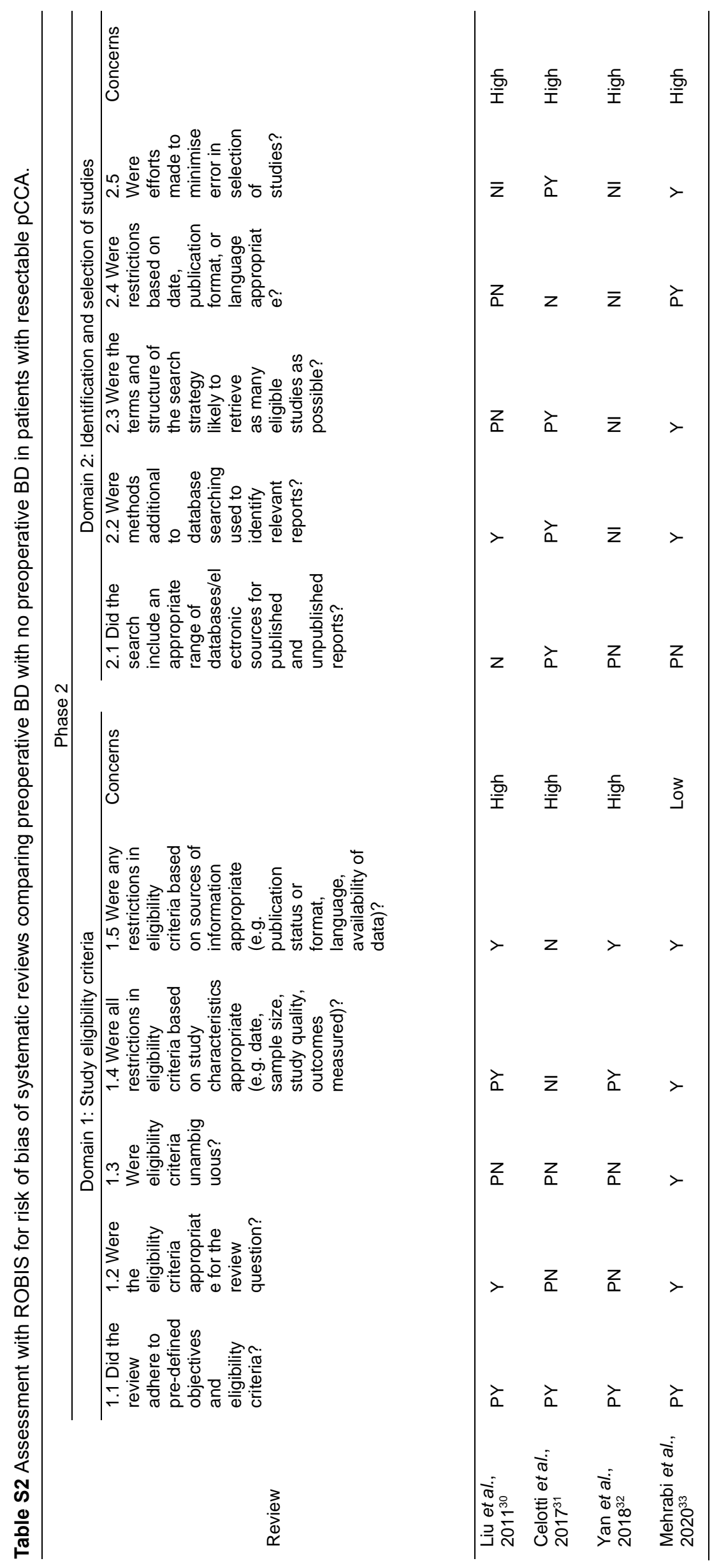


Preoperative biliary drainage in $\mathrm{pCCA}$






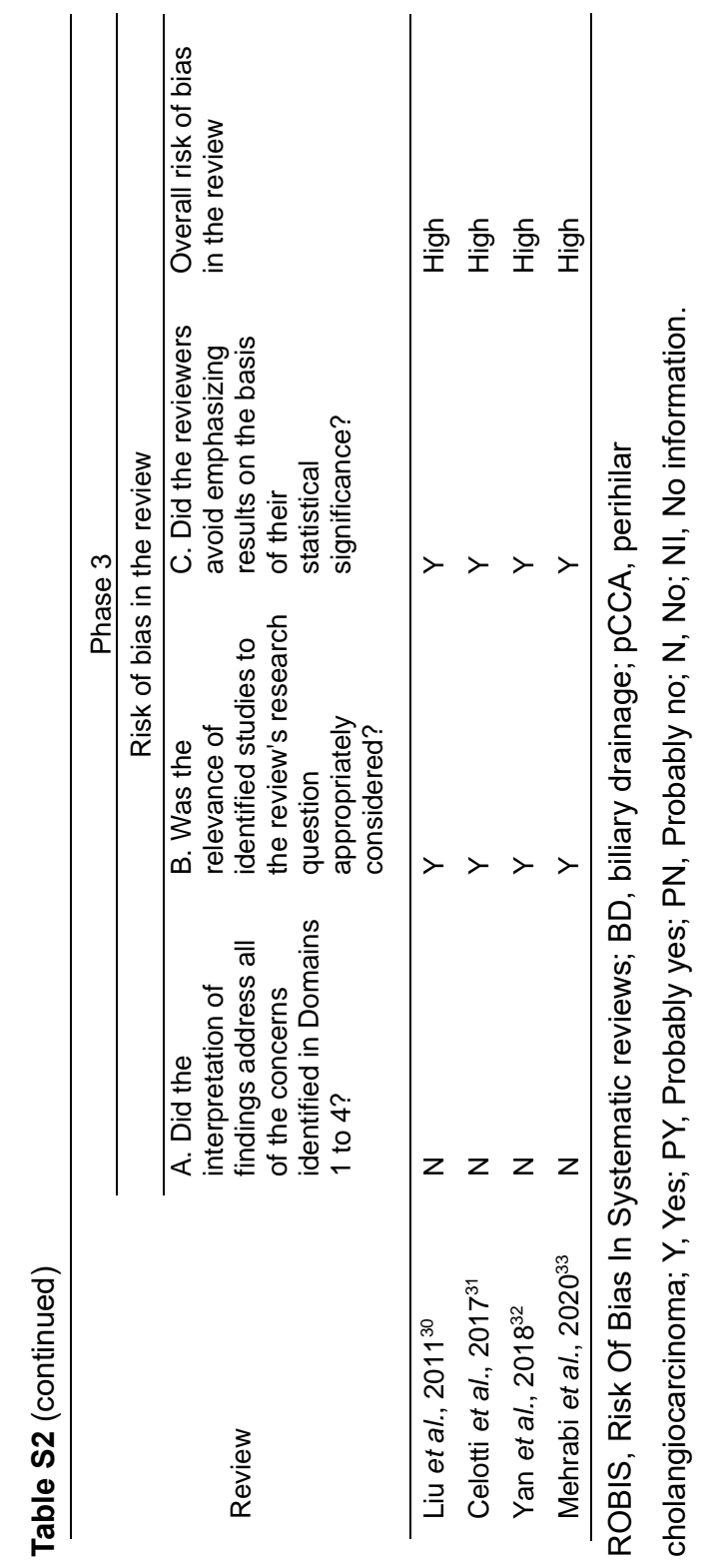


Preoperative biliary drainage in $\mathrm{pCCA}$

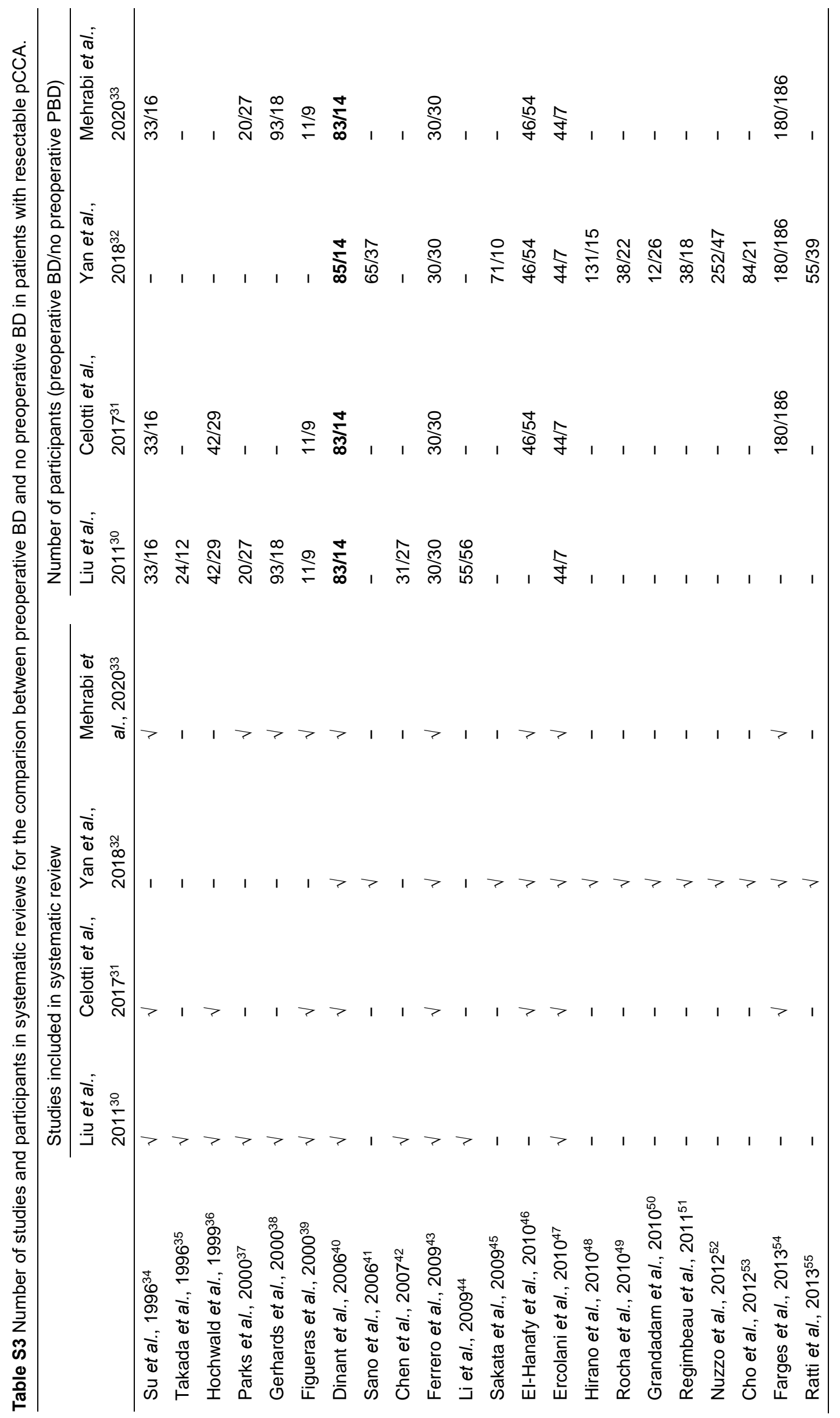


Chapter 2




Preoperative biliary drainage in $\mathrm{pCCA}$




Chapter 2




Preoperative biliary drainage in $\mathrm{pCCA}$









Preoperative biliary drainage in PCCA




Chapter 2

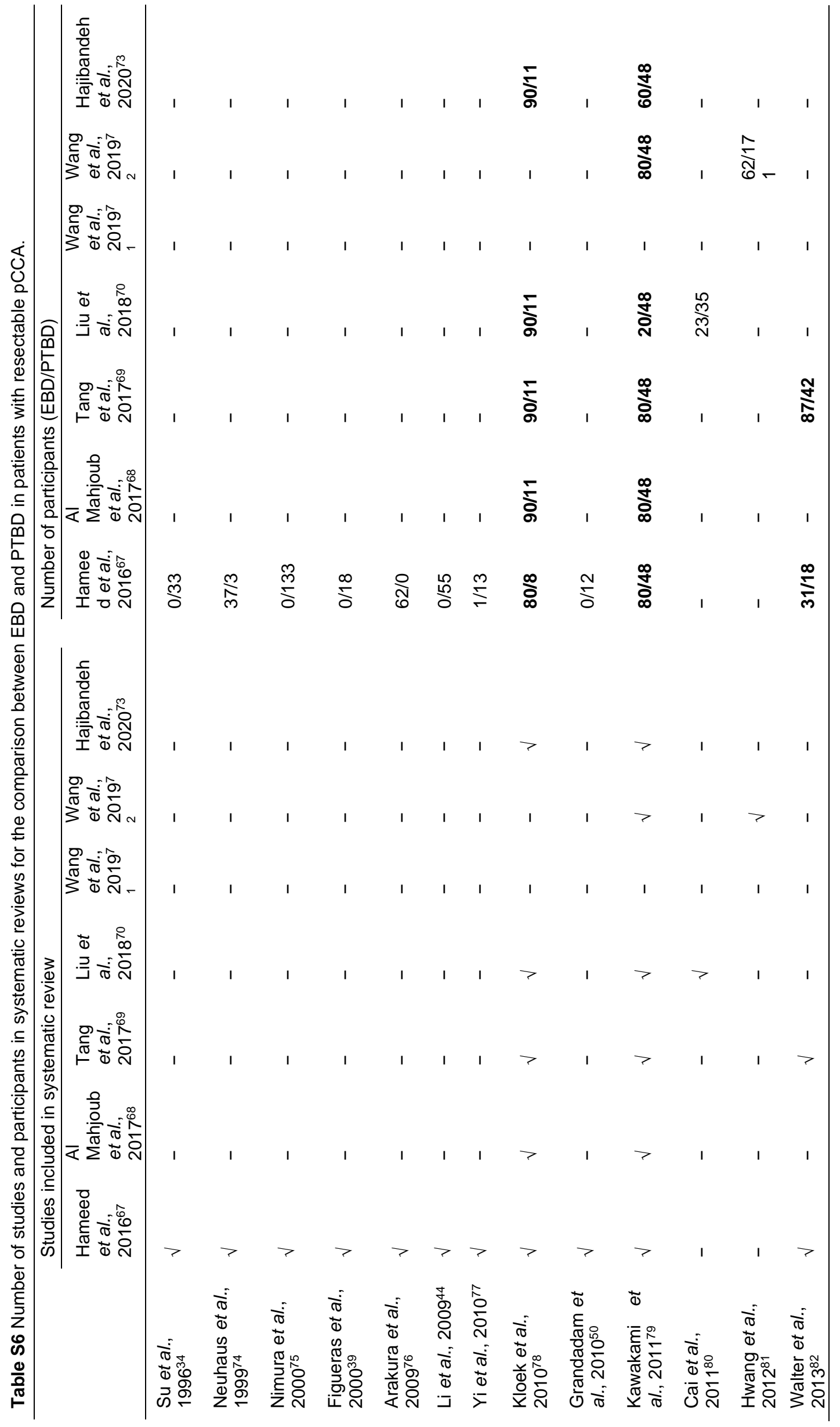


Preoperative biliary drainage in $\mathrm{PCCA}$




Chapter 2




Preoperative biliary drainage in $\mathrm{pCCA}$






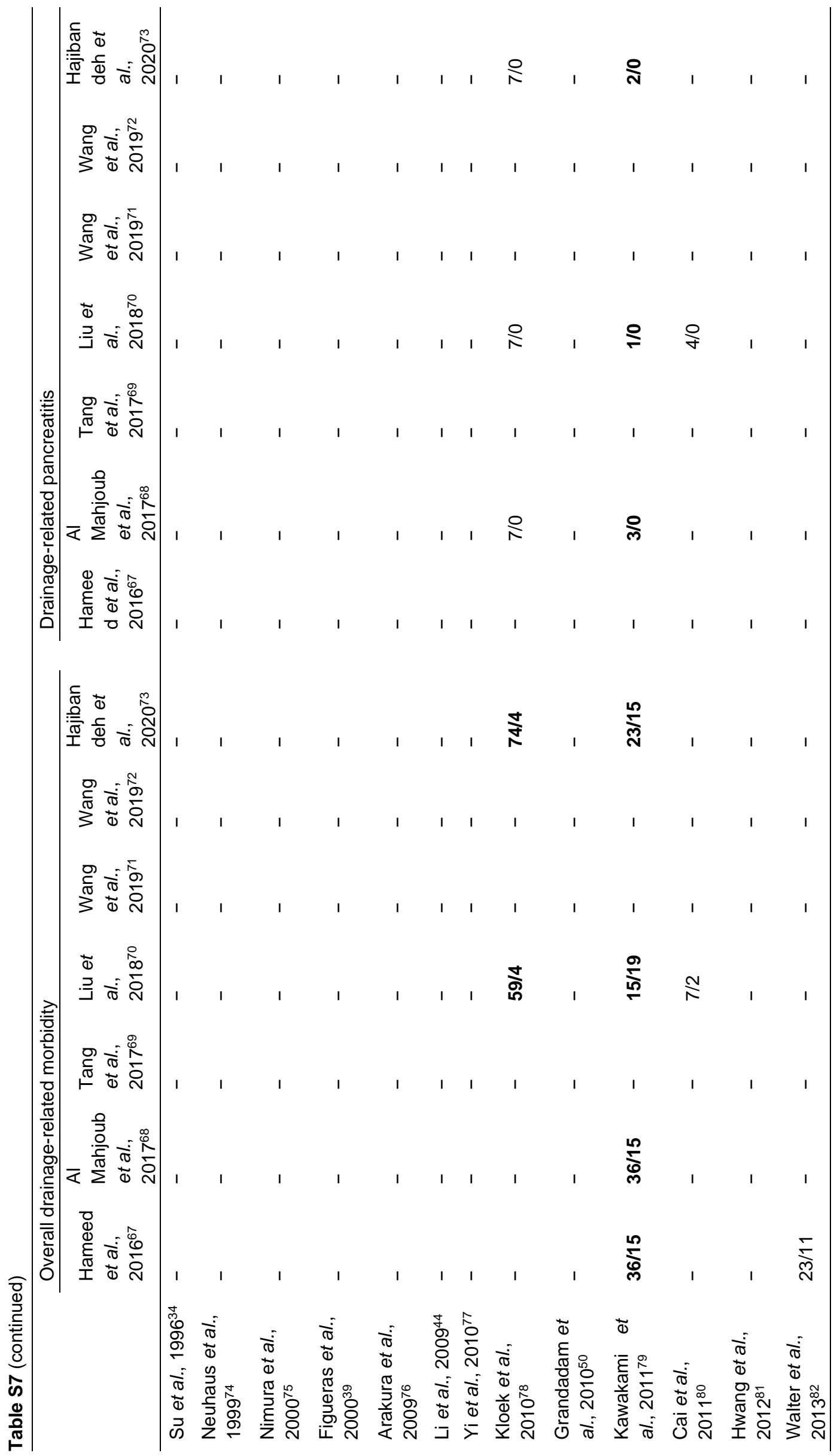


Preoperative biliary drainage in $\mathrm{pCCA}$









Preoperative biliary drainage in $\mathrm{pCCA}$

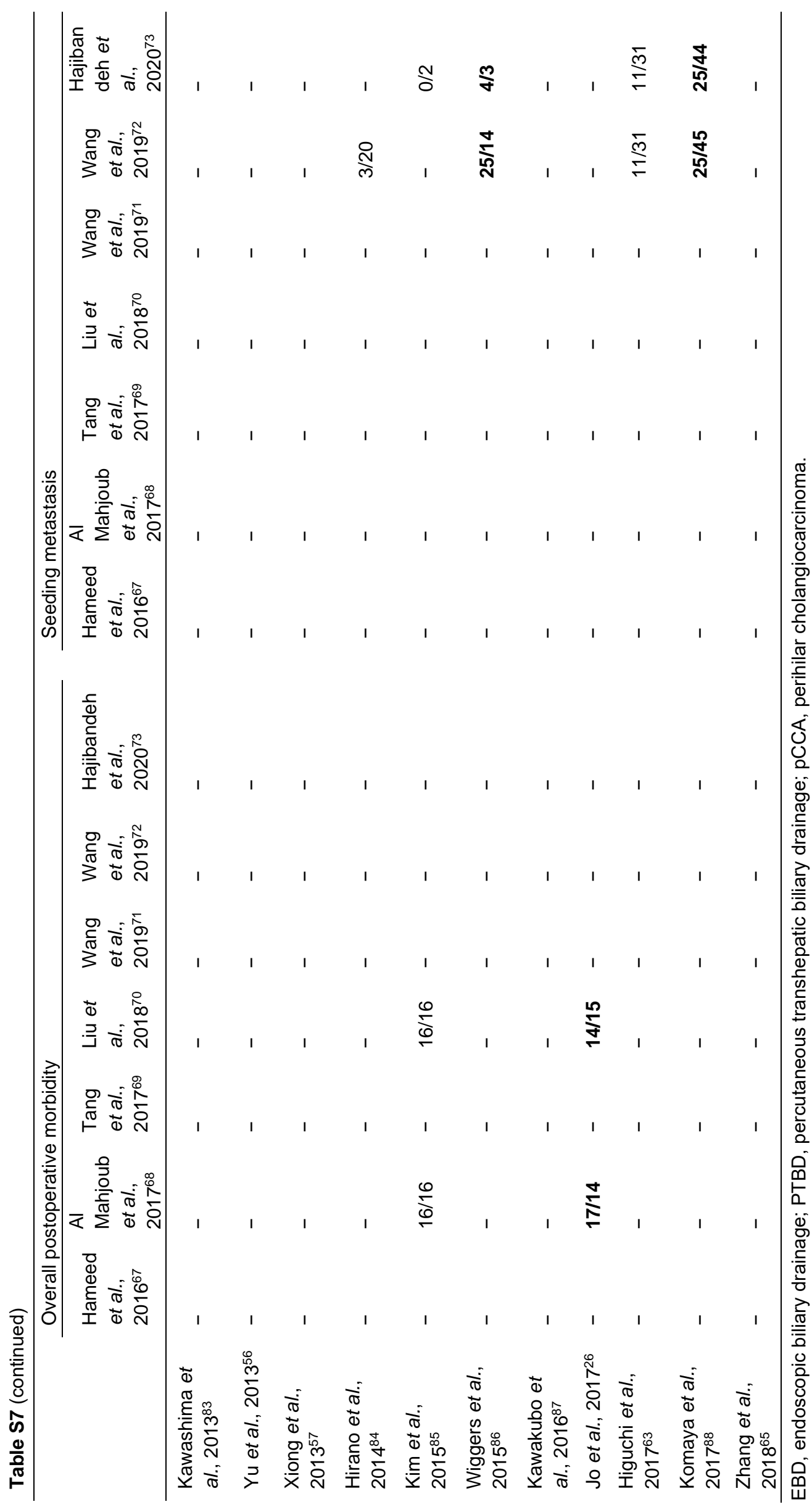




\section{Supplementary references}

1. Takada T. Is preoperative biliary drainage necessary according to evidence-based medicine? J Hepatobiliary Pancreat Surg. 2001;8:58-64.

2. Belghiti J, Ogata S. Preoperative optimization of the liver for resection in patients with hilar cholangiocarcinoma. HPB 2005;7:252-253.

3. Sakata J, Shirai Y, Wakai T, Nomura T, Sakata E, Hatakeyama K. Catheter tract implantation metastases associated with percutaneous biliary drainage for extrahepatic cholangiocarcinoma. World J Gastroenterol. 2005;11:7024-7027.

4. Briggs CD, Peterson M. Investigation and management of obstructive jaundice. Surgery. 2007;25:74-80.

5. Maguchi H, Takahashi K, Katanuma A, Osanai M, Nakahara K, Matuzaki S, et al. Preoperative biliary drainage for hilar cholangiocarcinoma. J Hepatobiliary Pancreat Surg. 2007;14:441-446.

6. Nagino M, Takada T, Miyazaki M, Miyakawa S, Tsukada K, Kondo S, et al. Preoperative biliary drainage for biliary tract and ampullary carcinomas. Journal of Hepato-Biliary-Pancreatic Surgery. 2008;15:25-30.

7. Nimura Y. Preoperative biliary drainage before resection for cholangiocarcinoma (Pro). HPB 2008;10:130-133.

8. van Delden OM, Lameris JS. Percutaneous drainage and stenting for palliation of malignant bile duct obstruction. Eur Radiol. 2008;18:448-456.

9. Kawakami H, Kondo S, Kuwatani M, Yamato H, Ehira N, Kudo T, et al. Preoperative biliary drainage for hilar cholangiocarcinoma: which stent should be selected? J Hepatobiliary Pancreat Sci. 2011;18:630-635.

10. Parodi A, Fisher D, Giovannini M, Baron T, Conio M. Endoscopic management of hilar cholangiocarcinoma. Nat Rev Gastroenterol Hepatol. 2012;9:105-112.

11. lacono C, Ruzzenente A, Campagnaro T, Bortolasi L, Valdegamberi A, Guglielmi A. Role of preoperative biliary drainage in jaundiced patients who are candidates for pancreatoduodenectomy or hepatic resection: highlights and drawbacks. Ann Surg. 2013;257:191-204.

12. Webb K, Saunders M. Endoscopic Management of Malignant Bile Duct Strictures. Gastrointest Endosc Clin N Am. 2013;23:313-331.

13. Yasuda I, Mukai T, Moriwaki H. Unilateral versus bilateral endoscopic biliary stenting for malignant hilar biliary strictures. Dig Endosc. 2013;25 Suppl 2:81-85.

14. Zhimin G, Noor H, Jian-Bo Z, Lin W, Rajiv Kumar J. Advances in diagnosis and treatment of hilar cholangiocarcinoma - A review. Med Sci Monit 2013;19:648-656.

15. Fang Y, Gurusamy KS, Wang Q, Davidson BR, Lin H, Xie X, et al. Meta-analysis of randomized clinical trials on safety and efficacy of biliary drainage before surgery for obstructive jaundice. $\mathrm{Br}$ J Surg. 2013;100:1589-1596.

16. Leng JJ, Zhang N, Dong JH. Percutaneous transhepatic and endoscopic biliary drainage for malignant biliary tract obstruction: a meta-analysis. World J Surg Oncol. 2014;12:272.

17. Paik WH, Loganathan N, Hwang JH. Preoperative biliary drainage in hilar cholangiocarcinoma: When and how? World J Gastrointest Endosc. 2014;6:68-73.

18. Soares KC, Kamel I, Cosgrove DP, Herman JM, Pawlik TM. Hilar cholangiocarcinoma: diagnosis, treatment options, and management. Hepatobiliary Surg Nutr. 2014;3:18-34.

19. Park DH. Endoscopic ultrasound-guided biliary drainage of hilar biliary obstruction. J 
Hepatobiliary Pancreat Sci. 2015;22:664-668.

20. Poruk KE, Pawlik TM, Weiss MJ. Perioperative Management of Hilar Cholangiocarcinoma. J Gastrointest Surg. 2015;19:1889-1899.

21. Rodarte-Shade $M$, Kahaleh $M$. Stent placement as a bridge to surgery in malignant biliary obstruction (pancreatic cancer, distal bile duct cancer, and hilar tumors). Gastrointestinal Intervention. 2015;4:21-26.

22. Rustagi T, Jamidar PA. Endoscopic treatment of malignant biliary strictures. Curr Gastroenterol Rep. 2015;17:426.

23. Saxena P, Kumbhari V, Zein ME, Khashab MA. Preoperative biliary drainage. Dig Endosc. 2015;27:265-277.

24. Tsuchikawa T, Hirano S, Okamura K, Matsumoto J, Tamoto E, Murakami S, et al. Advances in the surgical treatment of hilar cholangiocarcinoma. Expert Rev Gastroenterol Hepatol. 2015;9:369-374.

25. Umeda J, Itoi T. Current status of preoperative biliary drainage. J Gastroenterol 2015;50:940954.

26. Jo JH, Chung MJ, Han DH, Park JY, Bang S, Park SW, et al. Best options for preoperative biliary drainage in patients with Klatskin tumors. Surg Endosc. 2017;31:422-429.

27. Tringali A, Cintolo M, Sica M, Manta R. Endoscopic vs percutaneous preoperative biliary drainage in hilar cholangiocarcinoma: A systematic review and meta-analysis. Digestive and Liver Disease. 2017;49 (Supplement 2):e183.

28. Tang Z, Yang Y, Zhao Z, Wei K, Meng W, Li X. The clinicopathological factors associated with prognosis of patients with resectable perihilar cholangiocarcinoma: A systematic review and meta-analysis. Medicine. 2018;97 e11999.

29. Lee TH, Moon JH, Park SH. Biliary stenting for hilar malignant biliary obstruction. Dig Endosc. 2020;32:275-286.

30. Liu F, Li Y, Wei Y, Li B. Preoperative biliary drainage before resection for hilar cholangiocarcinoma: whether or not? A systematic review. Dig Dis Sci. 2011;56:663-672.

31. Celotti A, Solaini L, Montori G, Coccolini F, Tognali D, Baiocchi G. Preoperative biliary drainage in hilar cholangiocarcinoma: Systematic review and meta-analysis. Eur J Surg Oncol. 2017;43:1628-1635.

32. Yan K, Tian J, Xu J, Fu Y, Zhang H, Song J. The value of preoperative biliary drainage in hilar cholangiocarcinoma: A systematic review and meta analysis of 10 years' literatures. Int J Clin Exp Med. 2018;11:3462-3472.

33. Mehrabi A, Khajeh E, Ghamarnejad O, Nikdad M, Chang DH, Buchler MW, et al. Meta-analysis of the efficacy of preoperative biliary drainage in patients undergoing liver resection for perihilar cholangiocarcinoma. Eur J Radiol. 2020;125:108897.

34. Su CH, Tsay SH, Wu CC, Shyr YM, King KL, Lee CH, et al. Factors influencing postoperative morbidity, mortality, and survival after resection for hilar cholangiocarcinoma. Ann surg. 1996;223:384-394.

35. Takada T, Yasuda H. Value of pre-operative biliary drainage prior to bile duct cancer surgery: Results of a retrospective review. Asian J Surg. 1996;19:84-87,89.

36. Hochwald SN, Burke EC, Jarnagin WR, Fong Y, Blumgart LH. Association of preoperative biliary stenting with increased postoperative infectious complications in proximal cholangiocarcinoma. Arch Surg. 1999;134:261-266.

37. Parks RW, Currie EJ, Madhavan KK, Garden OJ. Increased bacterobilia associated with 
preoperative biliary drainage in patients with hilar cholangiocarcinoma. HPB. 2000;2:375-381.

38. Gerhards MF, van Gulik TM, de Wit LT, Obertop H, Gouma DJ. Evaluation of morbidity and mortality after resection for hilar cholangiocarcinoma-a single center experience. Surgery. 2000;127:395-404.

39. Figueras J, Llado L, Valls C, Serrano T, Ramos E, Fabregat J, et al. Changing strategies in diagnosis and management of hilar cholangiocarcinoma. Liver Transpl. 2000;6:786-794.

40. Dinant S, Gerhards MF, Rauws EA, Busch OR, Gouma DJ, van Gulik TM. Improved outcome of resection of hilar cholangiocarcinoma (Klatskin tumor). Ann Surg Oncol. 2006;13:872-880.

41. Sano T, Shimada K, Sakamoto Y, Yamamoto J, Yamasaki S, Kosuge T. One hundred two consecutive hepatobiliary resections for perihilar cholangiocarcinoma with zero mortality. Ann Surg. 2006;244:240-247.

42. Chen D, Peng B, Li S, Liang L, Yin X, Tang D, et al. Effect of preoperative biliary drainage on hilar cholangiocarcinoma in patients underwent resection operation. Zhongguo Shi Yong Wai Ke Za Zhi. 2007;27:805-808.

43. Ferrero A, Lo Tesoriere R, Vigano L, Caggiano L, Sgotto E, Capussotti L. Preoperative biliary drainage increases infectious complications after hepatectomy for proximal bile duct tumor obstruction. World J Surg. 2009;33:318-325.

44. Li S, Chen D, Liang L, Peng B, Yin X. The impact of preoperative biliary drainage on surgical morbidity in hilar cholangiocarcinoma patients. Chin J Surg. 2009;47:1134-1137.

45. Sakata J, Shirai Y, Tsuchiya Y, Wakai T, Nomura T, Hatakeyama K. Preoperative cholangitis independently increases in-hospital mortality after combined major hepatic and bile duct resection for hilar cholangiocarcinoma. Langenbecks Arch Surg. 2009;394:1065-1072.

46. El-Hanafy E. Pre-operative biliary drainage in hilar cholangiocarcinoma, benefits and risks, single center experience. Hepatogastroenterology. 2010;57:414-419.

47. Ercolani G, Zanello M, Grazi GL, Cescon M, Ravaioli M, Del Gaudio M, et al. Changes in the surgical approach to hilar cholangiocarcinoma during an 18-year period in a Western single center. J Hepatobiliary Pancreat Sci. 2010;17:329-337.

48. Hirano S, Kondo S, Tanaka E, Shichinohe T, Tsuchikawa T, Kato K, et al. Outcome of surgical treatment of hilar cholangiocarcinoma: a special reference to postoperative morbidity and mortality. J Hepatobiliary Pancreat Sci. 2010;17:455-462.

49. Rocha FG, Matsuo K, Blumgart LH, Jarnagin WR. Hilar cholangiocarcinoma: the Memorial Sloan-Kettering Cancer Center experience. J Hepatobiliary Pancreat Sci. 2010;17:490-496.

50. Grandadam S, Compagnon P, Arnaud A, Olivie D, Malledant Y, Meunier B, et al. Role of preoperative optimization of the liver for resection in patients with hilar cholangiocarcinoma type III. Ann Surg Oncol. 2010;17:3155-3161.

51. Regimbeau JM, Fuks D, Le Treut YP, Bachellier P, Belghiti J, Boudjema K, et al. Surgery for hilar cholangiocarcinoma: a multi-institutional update on practice and outcome by the AFC-HC study group. J Gastrointest Surg. 2011;15:480-488.

52. Nuzzo G, Giuliante F, Ardito F, Giovannini I, Aldrighetti L, Belli G, et al. Improvement in perioperative and long-term outcome after surgical treatment of hilar cholangiocarcinoma: results of an Italian multicenter analysis of 440 patients. Arch Surg. 2012;147:26-34.

53. Cho MS, Kim SH, Park SW, Lim JH, Choi GH, Park JS, et al. Surgical outcomes and predicting factors of curative resection in patients with hilar cholangiocarcinoma: 10-year single-institution experience. J Gastrointest Surg. 2012;16:1672-1679.

54. Farges O, Regimbeau JM, Fuks D, Le Treut YP, Cherqui D, Bachellier P, et al. Multicentre 
European study of preoperative biliary drainage for hilar cholangiocarcinoma. $\mathrm{Br} \mathrm{J}$ Surg. 2013;100:274-283.

55. Ratti F, Cipriani F, Ferla F, Catena M, Paganelli M, Aldrighetti LA. Hilar cholangiocarcinoma: preoperative liver optimization with multidisciplinary approach. Toward a better outcome. World J Surg. 2013;37:1388-1396.

56. Yu FX, Ji SQ, Su LF, Zhang SC, Zhang QY. Effectiveness and safety of preoperative percutaneous transhepatic cholangiodrainage with bile re-infusion in patients with hilar cholangiocarcinoma: a retrospective controlled study. Am J Med Sci. 2013;346:353-357.

57. Xiong JJ, Nunes QM, Huang W, Pathak S, Wei AL, Tan CL, et al. Preoperative biliary drainage in patients with hilar cholangiocarcinoma undergoing major hepatectomy. World J Gastroenterol. 2013;19:8731-8739.

58. Furusawa N, Kobayashi A, Yokoyama T, Shimizu A, Motoyama H, Miyagawa S. Surgical treatment of 144 cases of hilar cholangiocarcinoma without liver-related mortality. World J Surg. 2014;38:1164-1176.

59. Yan Y, Lu N, Tian W, Liu T. Evolution of surgery for Klatskin tumor demonstrates improved outcome: a single center analysis. Tumori. 2014;100:e250-256.

60. Ribero D, Zimmitti G, Aloia TA, Shindoh J, Fabio F, Amisano M, et al. Preoperative Cholangitis and Future Liver Remnant Volume Determine the Risk of Liver Failure in Patients Undergoing Resection for Hilar Cholangiocarcinoma. J Am Coll Surg. 2016;223:87-97.

61. Ito $\mathrm{Y}$, Nakai $\mathrm{Y}$, Isayama H, Tsujino T, Hamada T, Umefune G, et al. Impact of Preoperative Biliary Drainage on Surgical Outcomes in Periampullary and Hilar Malignancy: A Single-Center Experience. Surg Laparosc Endosc Percutan Tech. 2016;26:150-155.

62. Cai $\mathrm{Y}$, Tang Q, Xiong X, Li F, Ye H, Song P, et al. Preoperative biliary drainage versus direct surgery for perihilar cholangiocarcinoma: A retrospective study at a single center. Biosci Trends. 2017;11:319-325.

63. Higuchi R, Yazawa T, Uemura S, Izumo W, Furukawa T, Yamamoto M. ENBD is Associated with Decreased Tumor Dissemination Compared to PTBD in Perihilar Cholangiocarcinoma. J Gastrointest Surg. 2017;21:1506-1514.

64. Kimura N, Young AL, Toyoki Y, Wyatt JI, Toogood GJ, Hidalgo E, et al. Radical operation for hilar cholangiocarcinoma in comparable Eastern and Western centers: Outcome analysis and prognostic factors. Surgery. 2017;162:500-514.

65. Zhang XF, Beal EW, Merath K, Ethun CG, Salem A, Weber SM, et al. Oncologic effects of preoperative biliary drainage in resectable hilar cholangiocarcinoma: Percutaneous biliary drainage has no adverse effects on survival. J Surg Oncol. 2018;117:1267-1277.

66. Ratti F, Cipriani F, Fiorentini G, Hidalgo Salinas C, Catena M, Paganelli M, et al. Management of hilum infiltrating tumors of the liver: The impact of experience and standardization on outcome. Dig Liver Dis. 2019;51:135-141.

67. Hameed A, Pang T, Chiou J, Pleass H, Lam V, Hollands M, et al. Percutaneous vs. endoscopic pre-operative biliary drainage in hilar cholangiocarcinoma - a systematic review and metaanalysis. HPB 2016;18:400-410.

68. Al Mahjoub A, Menahem B, Fohlen A, Dupont B, Alves A, Launoy G, et al. Preoperative Biliary Drainage in Patients with Resectable Perihilar Cholangiocarcinoma: Is Percutaneous Transhepatic Biliary Drainage Safer and More Effective than Endoscopic Biliary Drainage? A Meta-Analysis. J Vasc Interv Radiol. 2017;28:576-582.

69. Tang Z, Yang Y, Meng W, Li X. Best option for preoperative biliary drainage in Klatskin tumor: A 
systematic review and meta-analysis. Medicine 2017;96:e8372.

70. Liu JG, Wu J, Wang J, Shu GM, Wang YJ, Lou C, et al. Endoscopic Biliary Drainage Versus Percutaneous Transhepatic Biliary Drainage in Patients with Resectable Hilar Cholangiocarcinoma: A Systematic Review and Meta-Analysis. J Laparoendosc Adv Surg Tech A. 2018;28:1053-1060.

71. Wang L, Lin N, Xin F, Zeng Y, Liu J. Comparison of long-term efficacy between endoscopic and percutaneous biliary drainage for resectable extrahepatic cholangiocarcinoma with biliary obstruction: A systematic review and meta-analysis. Saudi J Gastroenterol. 2019;25:81-88.

72. Wang L, Lin N, Xin F, Ke Q, Zeng Y, Liu J. A systematic review of the comparison of the incidence of seeding metastasis between endoscopic biliary drainage and percutaneous transhepatic biliary drainage for resectable malignant biliary obstruction. World J Surg Oncol. 2019;17:116.

73. Hajibandeh S, Hajibandeh S, Satyadas T. Endoscopic Versus Percutaneous Preoperative Biliary Drainage in Patients With Klatskin Tumor Undergoing Curative Surgery: A Systematic Review and Meta-Analysis of Short-Term and Long-Term Outcomes. Surg Innov. 2020;27:279-290.

74. Neuhaus P, Jonas S, Bechstein WO, Lohmann R, Radke C, Kling N, et al. Extended resections for hilar cholangiocarcinoma. Ann Surg. 1999;230:808-818.

75. Nimura Y, Kamiya J, Kondo S, Nagino M, Uesaka K, Oda K, et al. Aggressive preoperative management and extended surgery for hilar cholangiocarcinoma: Nagoya experience. J Hepatobiliary Pancreat Surg. 2000;7:155-162.

76. Arakura N, Takayama M, Ozaki Y, Maruyama M, Chou Y, Kodama R, et al. Efficacy of preoperative endoscopic nasobiliary drainage for hilar cholangiocarcinoma. J Hepatobiliary Pancreat Surg. 2009;16:473-477.

77. Yi B, Xu AM, Lai EC, Qu ZQ, Cheng QB, Liu C, et al. Preoperative portal vein embolization for hilar cholangiocarcinoma--a comparative study. Hepatogastroenterology. 2010;57:1341-1346.

78. Kloek JJ, van der Gaag NA, Aziz Y, Rauws EA, van Delden OM, Lameris JS, et al. Endoscopic and percutaneous preoperative biliary drainage in patients with suspected hilar cholangiocarcinoma. J Gastrointest Surg. 2010;14:119-125.

79. Kawakami H, Kuwatani M, Onodera M, Haba S, Eto K, Ehira N, et al. Endoscopic nasobiliary drainage is the most suitable preoperative biliary drainage method in the management of patients with hilar cholangiocarcinoma. J Gastroenterol. 2011;46:242-248.

80. Cai Y, Su S, Cui W, Fei L, Li J. Selection of preoperative biliary drainage procedure for resectable hilar cholangiocarcinoma. Chin J Gen Surg. 2011;20:844-847.

81. Hwang S, Song GW, Ha TY, Lee YJ, Kim KH, Ahn CS, et al. Reappraisal of percutaneous transhepatic biliary drainage tract recurrence after resection of perihilar bile duct cancer. World J Surg. 2012;36:379-385.

82. Walter T, Ho CS, Horgan AM, Warkentin A, Gallinger S, Greig PD, et al. Endoscopic or percutaneous biliary drainage for Klatskin tumors? J Vasc Interv Radiol. 2013;24:113-121.

83. Kawashima H, Itoh A, Ohno E, Itoh Y, Ebata T, Nagino M, et al. Preoperative endoscopic nasobiliary drainage in 164 consecutive patients with suspected perihilar cholangiocarcinoma: a retrospective study of efficacy and risk factors related to complications. Ann Surg. 2013;257:121127.

84. Hirano S, Tanaka E, Tsuchikawa T, Matsumoto J, Kawakami H, Nakamura T, et al. Oncological benefit of preoperative endoscopic biliary drainage in patients with hilar cholangiocarcinoma. $J$ Hepatobiliary Pancreat Sci. 2014;21:533-540.

85. Kim KM, Park JW, Lee JK, Lee KH, Lee KT, Shim SG. A Comparison of Preoperative Biliary 
Preoperative biliary drainage in $\mathrm{PCCA}$

Drainage Methods for Perihilar Cholangiocarcinoma: Endoscopic versus Percutaneous Transhepatic Biliary Drainage. Gut Liver. 2015;9:791-799.

86. Wiggers JK, Groot Koerkamp B, Coelen RJ, Doussot A, van Dieren S, Rauws EA, et al. Percutaneous Preoperative Biliary Drainage for Resectable Perihilar Cholangiocarcinoma: No Association with Survival and No Increase in Seeding Metastases. Ann Surg Oncol. 2015;22 Suppl 3:S1156-1163.

87. Kawakubo K, Kawakami H, Kuwatani M, Haba S, Kudo T, Taya YA, et al. Lower incidence of complications in endoscopic nasobiliary drainage for hilar cholangiocarcinoma. World $\mathrm{J}$ Gastrointest Endosc. 2016;8:385-390.

88. Komaya K, Ebata T, Yokoyama Y, Igami T, Sugawara G, Mizuno T, et al. Verification of the oncologic inferiority of percutaneous biliary drainage to endoscopic drainage: A propensity score matching analysis of resectable perihilar cholangiocarcinoma. Surgery. 2017;161:394-404. 



\section{Part II}

The role of bile salt signaling in liver regeneration 



\section{Chapter 3}

Precision-cut liver slices as a model to study human liver regeneration 


\begin{abstract}
Background: Fibroblast growth factor 19 (FGF19) activates signal transducer and activator of transcription 3 (STAT3) signaling in hepatocytes through induction of interleukin 6 (IL6) production by innate immune cells, driving malignant proliferation of mouse hepatocytes. Here we aimed to explore whether FGF19 activates the IL6/STAT3 axis to promote hepatocyte proliferation in the human precision-cut liver slices (hPCLS) model, and to assess the suitability of hPCLS as an ex vivo model to study human liver regeneration.
\end{abstract}

Methods: hPCLS were derived from tumor-distal liver tissue from patients who underwent partial liver resection for colorectal liver metastases and incubated with vehicle, lipopolysaccharide (LPS), recombinant FGF19, nuclear bile salt receptor agonists (OCA, FXR agonist; CITCO, CAR agonist; rifampicin, PXR agonist). 5C and Geltrex were used to assess their effectiveness in maintaining expression of genes related to a differentiated hepatocyte phenotype. mRNA expression of IL6, FXR/FGF19 pathway-related genes and proliferation-related genes were assessed. IL6 and FGF19 concentrations in the conditioned medium were determined.

Results: FGF19 did not induce IL6 mRNA expression in hPCLS. In addition, marked phosphorylation of ERK1/2 was already observed in untreated liver slices. FGF19 had no additional effect on levels of phosphorylated ERK1/2. Expression of FGF19 pathway-related genes (CYP7A1, FGFR4, KLB, PCK1) rapidly declined upon hPCLS culturing. The use of $5 \mathrm{C}$ and Geltrex did not maintain expression of these genes. Furthermore, nuclear receptors agonists OCA and rifampicin did not induce cell proliferation in hPCLS.

Conclusions: FGF19 and CAR treatment did not result in cell proliferation in hPCLS. However, the efficacy of FGF19 and CAR treatment could not be ascertained in these exploratory experiments. Hence, questions regarding the involvement of the FGF19/IL6 axis in hepatocellular proliferation, and augmentation of liver growth by CAR agonism could not be answered with the current method of hPCLS source, preparation and culturing. 


\section{Introduction}

The robust capacity of the liver to regenerate allows a large removal $(70 \%)$ of liver mass for the curative goal of liver tumors. Insufficient liver remnant after hepatic resection can lead to small for size syndrome, where patients present with impaired liver function 1,2 . The remnant liver volume and its functionality are important for successful regeneration, especially when there is pre-existing damaged liver parenchyma ${ }^{3,4}$. An effective pharmaceutical therapy to accelerate liver regeneration in patients undergoing liver resection is currently lacking.

Bile salts activate a dedicated nuclear receptor farnesoid $X$ receptor (FXR) to maintain bile salt homeostasis through regulation of hepatic synthesis and transport of bile salts 5 . Dysregulation of bile salt homeostasis can result in hepatic bile salt accumulation and attendant hepatocellular toxicity. Bile salts are also involved in the process of liver regeneration after partial hepatectomy $(\mathrm{PH})$, as liver regeneration is delayed in $F x r$ deficient mice ${ }^{6}$. One role of FXR in liver regeneration might be the release of mitogenic fibroblast growth factor 19 (FGF19, Fgf15 in rodents). Fgf15 ${ }^{-/-}$mice showed delayed liver regeneration and considerable mortality (about $70 \%$ ) after $70 \% \mathrm{PH}^{7}$.

The cytokine interleukin 6 (IL-6) activates JAK/STAT, MAPK and PI3K/AKT signaling cascades, to control the early regenerative response after $\mathrm{PH}^{8}$. FGF19 can activate signal transducer and activator of transcription 3 (STAT3) signaling in hepatocytes through induction of IL-6 production by non-parenchymal cells, likely Kupffer cells. This non-cell autonomous FGF19-IL6 axis was shown to drive malignant proliferation of mouse hepatocytes ${ }^{9}$. It is conceivable that effects of bile salts on liver regeneration are via bile salt homeostatic actions, as well as via an FXR-FGF19-IL6 signaling axis.

The liver contains parenchymal and non-parenchymal cells. The latter consists of various immune cells (e.g. macrophages, natural killer cells, dendritic cells), hepatic stellate cells, bile duct epithelial cells and other cell types ${ }^{10}$. Although isolated hepatocytes are widely used for the research of liver processes, it fails to imitate the cell-cell interactions occurring in situ, whereas this interaction is important for liver regeneration ${ }^{11,12}$. For example, angiocrine factors derived from the liver sinusoidal endothelial cells trigger hepatocyte growth in mice ${ }^{13}$. Precision-cut liver slices (PCLS) preserve all cell types, where the cell-cell interaction and cell heterogeneity remain in the original liver matrix ${ }^{14,15}$. PCLS were first used to study liver regeneration by Tschuor at al., who demonstrated increased numbers of hepatocytes positive for the 
proliferative marker phosphorylated histone 3 , following treatment of murine and human PCLS with an agonist of the constitutive androstane receptor (CAR ${ }^{2}$. Thus far, these initial findings (note that observations were based on PCLS from a single patient) -and use of PCLS as ex vivo model of liver regeneration- have not been confirmed or extended.

Most studies on the role of FGF19 and bile salts in liver regeneration employed animal models, and the empirical support for conservation of these functions in human is scarce ${ }^{6,7,16}$. In this study we aimed to explore whether FGF19 activates the IL6/STAT3 axis to promote hepatocyte proliferation in the human PCLS (hPCLS) model. Further aim was to reproduce and extend the potential of specific nuclear receptor agonists, including FXR activators, to stimulate hepatocyte growth in hPCLSs. These were exploratory studies, with main goal to assess the suitability of hPCLS as an ex vivo model to study liver regeneration and pharmaceutical modulation thereof.

\section{Materials and Methods}

\section{Patient materials}

Tumor-distal liver tissue (ca. $4 \times 3 \times 2 \mathrm{~cm}$ ) was obtained from patients who underwent partial liver resection for colorectal liver metastases, at the beginning of the resection procedure (open or laparoscopic). The edge of segment 4 was sampled by the surgeon using surgical scissors and judged by the surgeon to be macroscopically devoid of tumor. Liver specimens of a total of 10 patients were processed to hPCLS for below exploratory studies. All patients provided written informed consent. This study was approved by the Medical Ethical Committee of Maastricht University Medical Center (METC\# 14-4-153). Following excision of liver tissue in the operating room, the liver tissues were immediately stored in ice-cold University of Wisconsin organ preservation solution (UW) and transported to the laboratory.

\section{Chemicals}

Lipopolysaccharide (LPS, from Escherichia coli O55:B5, \#L2880, Sigma, Darmstadt, Germany), FGF19 (\#969-FG, R\&D Systems, Minnesota, US), obeticholic acid (OCA) (Intercept Pharmaceuticals, New York, USA), CITCO (6-(4-Chlorophenyl)imidazo[2,1b][1,3]thiazole-5-carbaldehyde O-(3,4-dichlorobenzyl)oxime) (R\&D Systems, Minnesota, US), and rifampicin (Sigma, Darmstadt, Germany) were obtained from the 
indicated suppliers. Forskolin (FSK) (Sigma, Darmstadt, Germany), transforming growth factor $\beta$ inhibitor SB431542 (SB43) (Tocris, Abingdon, UK), DAPT (N-[N-(3,5Difluorophenacetyl)-L-alanyl]-S-phenylglycine t-butyl ester) (Sigma, Darmstadt, Germany), IWP2 (N-(6-Methyl-2-benzothiazolyl)-2-[(3,4,6,7-tetrahydro-4-oxo-3phenylthieno[3,2-d]pyrimidin-2-yl)thio]-acetamide) (Tocris, Abingdon, UK), bone morphogenetic protein inhibitor LDN193189 (Tocris, Abingdon, UK), PMA (phorbol 12myristate 13-acetate) (R\&D Systems, Minnesota, US), and dimethyl sulfoxide (DMSO) (molecular biology grade, D8418, Sigma, Darmstadt, Germany) were purchased from the indicated suppliers.

\section{Preparation of precision cut liver slices}

hPCLS were prepared as described previously ${ }^{15}$. Briefly, cylindrical cores of the freshly sampled liver ( $6 \mathrm{~mm}$ diameter) were made using a dermal biopsy punch. Liver cores were sectioned to slices of approximately $250 \mu \mathrm{m}$ thickness with a Krumdieck tissue slicer (Alabama Research and Development, Munford, AL, USA) filled with oxygenated ice-cold Krebs-Henseleit Buffer (KHB, $5 \mathrm{mM} \mathrm{NaCl}, 118 \mathrm{mM} \mathrm{KCl}, 1.1 \mathrm{mM} \mathrm{MgSO}$, 1.2 $\mathrm{mM} \mathrm{KH}_{2} \mathrm{PO}_{4}, 25 \mathrm{mM} \mathrm{NaHCO}_{3}, 2.5 \mathrm{mM} \mathrm{CaCl}_{2}, 25 \mathrm{mM}$ D-Glucose, $9.0 \mathrm{mM}$ HEPES in ultrapure water, $\mathrm{pH}$ 7.42). Liver slices were cultured in William's E Medium (Gibco, Paisley, Scotland) supplemented with $2.75 \mathrm{mg} / \mathrm{mL}$ D-glucose and $50 \mu \mathrm{g} / \mathrm{mL}$ gentamicin in 12-well plates at $37^{\circ} \mathrm{C}$ in an incubator containing $95 \% \mathrm{O}_{2}$ and $5 \% \mathrm{CO}_{2}$. Plates were continuously shaken on a rocking platform ( $80 \mathrm{rpm}$, transversal motion). After $1 \mathrm{~h}$ of recovery from the hPCLS procedure, medium was refreshed and the liver slices were exposed to various treatments. Prior studies revealed that liver slices remain viable (i.e. ATP levels were maintained) for at least $120 \mathrm{hrs}$ in William's E Medium ${ }^{17}$.

Treatments consisted of vehicle, $100 \mathrm{ng} / \mathrm{mL}$ LPS (dissolved in PBS/0.1\%BSA), 1-100 $\mathrm{ng} / \mathrm{ml} \mathrm{FGF19} \mathrm{(dissolved} \mathrm{in} \mathrm{PBS/0.1 \% BSA),} 10 \mu \mathrm{M}$ obeticholic acid (OCA, dissolved in DMSO; FXR agonist), $10 \mu \mathrm{M}$ CITCO (dissolved in DMSO, CAR agonist), $25 \mu \mathrm{M}$ rifampicin (dissolved in DMSO, PXR agonist) for the indicated time. For the treatment with these nuclear receptor agonists, reported experimental conditions were exactly replicated and included culturing hPCLS in 6 well plates on $0.4 \mu \mathrm{m}$ inserts (\#PICM0RG50, 30 mm diameter, Millicell) as described ${ }^{2}$. Supernatants of conditioned medium (centrifugation $1000 \mathrm{rpm}, 5$ minutes) were collected and stored at $-20^{\circ} \mathrm{C}$ for eventual analysis by ELISA. hPCLS were snap frozen in liquid nitrogen and stored at $-80^{\circ} \mathrm{C}$ for RNA and protein extraction. 


\section{Reverse transcription quantitative polymerase chain reaction (RT-qPCR)}

Total RNA was isolated from hPCLS using TRIzol reagent. A total of $750 \mathrm{ng}$ RNA was used for reverse transcription with SensiFAST ${ }^{T M}$ CDNA Synthesis Kit. For the qPCR, we used SensiFASTTM SYBR Hi-ROX kit, primers, and CDNA as template using a LightCycler ${ }^{\circledR} 480$ SW 1.5 system according to the manufacturer's protocol. LinRegPCR program was used to analyze qPCR data ${ }^{18}$. Gene expression levels were normalized using $36 B 4$ as reference gene.

\section{Enzyme linked immunosorbent assay (ELISA)}

IL6 levels in the conditioned medium were determined by sandwich ELISA as described ${ }^{19}$. Briefly, microtiter plates were coated with mouse anti-human IL-6 antibody. Samples and recombinant human IL6 standards were diluted in PBS containing $0.1 \%$ bovine serum albumin. Biotinylated rabbit anti-human IL6 antibody was used to detect captured antigen and streptavidin-horseradish peroxidase as a secondary detection reagent. We used tetramethylbenzidine as chromogenic substrate. FGF19 levels in the conditioned medium were assessed by sandwich ELISA as reported previously 20 .

\section{Western blotting}

For testing the activation (i.e. phosphorylation) of the ERK pathway, hPCLS were treated with vehicle (0.1\% DMSO), $100 \mathrm{nM}$ PMA, and $100 \mathrm{ng} / \mathrm{mL}$ FGF19 for different time points. Phosphorylated ERK1/2 protein expression was assessed by Western blotting. hPCLS were lysed using RIPA lysis buffer containing protease inhibitor cOmplete Mini (\#30496700, Roche, Mannheim, Germany) and phosphatase inhibitor PhosSTOP (\#4906845001, Roche, Mannheim, Germany) in a mini bead-beater (SUNON, China) in $2.0 \mathrm{~mL}$ screw cap vials containing glass beads, for three cycles of 10 seconds at maximum speed. Tissue homogenates were spun down for $1 \mathrm{~min}$ at $10000 \mathrm{~g}$, and supernatants were transferred to fresh tube. Total protein in homogenates was determined by BCA protein assay with bovine serum albumin as standard. A total of $100 \mu \mathrm{g}$ protein was separated on a $10 \%$ sodium dodecyl sulfate polyacrylamide gel. Proteins were transferred to nitrocellulose membrane which was incubated with primary antibody (cat nr\# 4695, Cell Signaling Technology, Massachusetts, US) and secondary detection antibody. Immunoreactive bands were visualized with SuperSignal West Pico Chemiluminescent Substrate Kit (Thermo Scientific, Rockford, 
US).

\section{Statistical analysis}

Data are presented as median [IQR]. Nonparametric Mann-Whitney U test or KruskalWallis test was used to test for differences between groups. Longitudinal changes were evaluated by Friedman test, with Dunn's multiple comparison test to compare differences between baseline and subsequent individual time points. Four slices were included per treatment condition or time point. Experiments were repeated three times for treatment with FGF19 and CITCO in hPCLS. A p value of less than 0.05 was considered statistically significant. All statistical analyses were performed using IBM SPSS 24.0, and all graphs were made using GraphPad Prism 8.

\section{Results}

\section{FGF19 does not increase IL6 mRNA expression in hPCLS}

To address whether FGF19 induces IL6 production in the hPCLS model, slices were treated with FGF19 and LPS as a positive control, for 20 and $44 \mathrm{hrs.} \mathrm{LPS} \mathrm{stimulation}$ increased the mRNA expression of IL6 after $20 \mathrm{~h}$ and $44 \mathrm{~h}(\mathrm{P}<0.05, \mathrm{P}<0.01$, respectively) (Fig. 1A-B). Consistently, the IL6 levels in the medium were elevated after LPS treatment for $20 \mathrm{~h}$ and $44 \mathrm{~h}(\mathrm{P}<0.05, \mathrm{P}<0.01$, respectively) (Fig. 1C-D). FGF19 did not affect the mRNA expression of IL6. To evaluate whether FGF19 induces cell proliferation in hPCLS, we tested expression of STAT3 downstream targets, including genes related to cell proliferation (CCND1 and PCNA) and suppression of apoptosis (BIRC5). FGF19 did not alter the expression of CCND1, PCNA and BIRC5 (Fig. 1A-B). The FGF19 target gene CYP7A1 was not detectable after culturing $20 \mathrm{~h}$ and $44 \mathrm{~h}$, and could not be used as a transcriptional read-out to verify proper FGF19 action. In addition, FGF19 did not increase IL6 levels in the conditioned medium after $20 \mathrm{~h}$ and $44 \mathrm{~h}$ incubation (Fig. 1C-D). IL6 levels induced by LPS treatment were apparently insufficient for paracrine activation of STAT3 signaling, as inferred from lack of effects on CCND1, PCNA and BIRC5. 
A



c



B



D



Figure 1. FGF19 does not induce IL6 mRNA expression in hPCLS after $20 \mathrm{hrs}$ incubation. hPCLS were incubated with vehicle, FGF19 or LPS for 20 and 44 hrs. Conditioned media were harvested and assayed for IL6 protein level. RNA was isolated from hPCLS and gene expression was analyzed by RT-qPCR. LPS treatment increased IL6 mRNA expression and protein levels after $20 \mathrm{~h}(\mathbf{A}, \mathbf{C})$ and $44 \mathrm{~h}(\mathbf{B}, \mathbf{D})$. FGF19 does not induce IL6 mRNA expression (A, B). Four slices were included per condition. The experiment for LPS/FGF19 treatment was replicated three times using slices derived from different patients $(\mathbf{A}, \mathbf{B})$. The dose-response experiment was performed once (C, D). A representative experiment is depicted. Differences among groups were analyzed with Kruskal-Wallis test, with Dunn's multiple comparison test to compare differences between control and specific treatment. Data is depicted as median [IQR]. Asterisks indicate significance levels: ${ }^{*} P<0.05$.

\section{FGF19 does not induce enhanced activation of ERK1/2}

Since FGF19 treatment did not result in altered expression of the tentative target gene IL6, we next tested responsiveness of slices to FGF19 treatment using a nontranscriptional read-out, i.e. activation of the extracellular signal-related kinase $[E R K] 1 / 2$ pathway, a mediator of FGF19 signaling ${ }^{9}$. In unstimulated slices, marked phosphorylation of ERK1/2 was already observed. FGF19 treatment for 5 to 60 minutes did not result in enhanced phosphorylation of ERK1/2 (Fig. 2). Likewise, the positive control PMA did not lead to higher levels of pERK1/2 in comparison to the vehicle control (DMSO). This suggests that the time to recover from slice preparation 
stress is insufficient to inactivate (e.g. dephosphorylate) the extracellular signalregulated kinase ERK1/2, and cellular 'stress' is still being experienced.



Figure 2. FGF19 does not enhance phosporylation of ERK1/2 in hPCLS. hPCLS were incubated with vehicle, FGF19 or PMA for 0-60 minutes. Phosphorylation of ERK1/2 was determined by Western blotting. FGF19 $(100 \mathrm{ng} / \mathrm{mL})$, DMSO (0.1\%) (vehicle control), PMA (100 $\mathrm{nM}$ ) (positive control) were used to treat hPCLS. The experiment was replicated two times using slices derived from two patients.

\section{Change of FGF19 pathway-related genes expression during culture in hPCLS}

Since no effects of FGF19 on IL6 mRNA expression or phosphorylation of ERK1/2 were observed, we next explored if mRNA expression of FGF19 pathway-related genes altered during slice culturing. hPCLS were cultured in the medium with no additions for up to $19 \mathrm{~h}$. During the first $1 \mathrm{~h}$ of pre-incubation (i.e. the recovery period), the expression of FXR, FGFR4 and KLB (FGF19 receptors), CYP7A1 and PCK1 (both negatively regulated by FGF19) rapidly declined (Fig. 3A). In contrast, FGF19 was initially below detection limits, but was readily detectable at the mRNA and protein level (data not shown) after $19 \mathrm{hrs}$. The expression of CYP7A1 further decreased after $9 \mathrm{~h}$, and became undetectable (i.e. could not be reliably quantified due to $\mathrm{Cq}>35$ ) after $19 \mathrm{~h}$. The median $\mathrm{Cq}$ of tested genes at different time points are summarized in Fig. 3B.

\section{Use of 5C and Geltrex does not improve FXR/FGF19 related genes expression}

In the experiment reported in Fig. 3, we observed a rapid decline of genes related to a differentiated hepatocyte phenotype such as the bile salt synthetic gene CYP7A1, which we use as a read-out for FGF19 signaling and becomes undetectable after 19 hrs of culturing. Dedifferentiated human hepatocytes lose some of the mature liver 



B



Figure 3. Time course of FGF19 pathway-related genes during culturing. hPCLS were cultured in the medium with no additions for up to $19 \mathrm{~h}$. RNA was isolated from hPCLS and gene expression was analyzed by RT-qPCR. T0=start recovery, $\mathrm{T} 1=$ =end recovery and medium refreshment. The change of FGF19 pathway-related genes during culturing (A). The median $\mathrm{Cq}$ of genes at different time points (B). Data was derived from a single patient, with 4 slices per time point.. Longitudinal changes were evaluated by Friedman test, with Dunn's multiple comparison test to compare differences between baseline (T0) and subsequent individual time points. Data is depicted as median [IQR]. Asterisks indicate significance levels: ${ }^{*} P<0.05$, ${ }^{* *} \mathrm{P}<0.01$.

functions which may limit their usefulness as an in vitro model to study liver pathophysiology 21,22 . It has been reported that a cocktail of five chemicals (5C) preserves differentiated function in isolated human hepatocytes ${ }^{23}$. To evaluate effectiveness of this cocktail in preserving function in hPCLS, we compared slices cultured under regular and 5C-supplemented conditions. Likewise, we explored whether embedding in basement membrane matrix (i.e. Geltrex) improved expression of genes associated with a differentiated phenotype, in particular genes regulated by FGF19 signaling. Geltrex, also employed in 3D cell culture models, can maintain 
viability of primary human hepatocytes and prolong metabolizing enzyme activities (e.g. CYP3A4, CYP1A2) ${ }^{24}$.

We thus tested whether $5 \mathrm{C}$ and/or Geltrex maintained expression of FXR/FGF19 pathway-related genes during culturing for $24 \mathrm{hrs}$. Unfortunately, mRNA expression of CYP7A1 was still not detectable in any of the groups (Fig. 4), whereas its expression was present in parent liver tissue $(\mathrm{Cq}=30.0)$. Similar to the earlier experiment (Fig. 3), a general decrease in expression of tested genes was observed when compared with parent liver tissue not subjected to slicing/culturing (Fig. 4). The experimental treatments $(5 \mathrm{C}$, Geltrex) failed to prevent this general decline, although expression of the drug metabolizing enzyme CYP3A4 was induced 2.0 fold by $5 \mathrm{C}$.

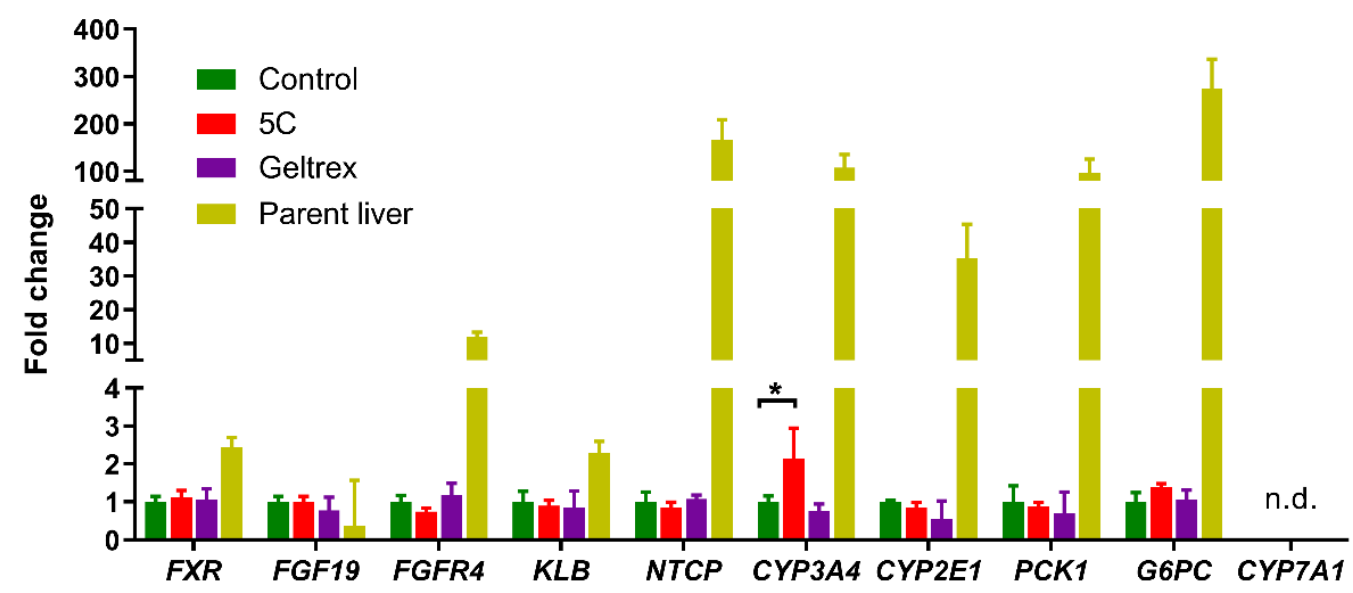

Figure 4. Use of 5C and Geltrex did not prevent the decline of FXR/FGF19-pathway related genes. hPCLS were cultured with vehicle, $5 \mathrm{C}$ or Geltrex for $24 \mathrm{~h}$. RNA was isolated from hPCLS and gene expression was analyzed by RT-qPCR. Four slices were included per condition, the experiment was conducted once. Data is depicted as median [IQR]. Asterisks indicate significance levels: ${ }^{*} P<0.05$. n.d., not detectable.

\section{Selected nuclear receptors agonists do not induce hepatocytes proliferation in hPCLS}

Activation of CAR was previously reported to stimulate hepatocyte proliferation in PCLS derived from mouse liver, as well as from a single patient ${ }^{2}$. To replicate and extend this finding, hPCLS were treated with agonists for nuclear bile salt receptors FXR (OCA), PXR (Rifampicin) and CAR (CITCO), the latter two being promiscuous receptors that can be activated by a limited number of bile salt species. As all three 
receptors are ligand-activated transcription factors, a transcriptional response was used as read-out for receptor activation, as reported previously ${ }^{2}$. Rifampicin induced mRNA expression of the PXR target gene CYP3A4 (Fig. 5A). No notable induction of CAR target genes CYP2E1 was observed upon treatment with CITCO (Fig. 5A). FXR target FGF19 was induced at transcriptional and protein levels by OCA treatment (Fig. 5A-B). Forkhead box M1 (FOXM1B) is a transcription factor involved in control of cell proliferation, and can regulate the expression of cell cycle gene cyclin D1 (CCND1). Treatment of hPCLS with OCA, Rifampicin or CITCO did not induce the mRNA expression of proliferation related genes FOXM1B, CCND1 and PCNA (Fig. 5C).


Figure 5. Selected nuclear receptors agonists do not induce cellular proliferation in hPCLS. hPCLS were cultured with vehicle, rifampicin, OCA, or CITCO for $44 \mathrm{~h}$. RNA was isolated from hPCLS and gene expression was analyzed by RT-qPCR. Conditioned media were harvested and assayed for FGF19 protein level. Rifampicin induced mRNA expression of CYP3A4 (A). OCA induced FGF19 expression at transcriptional and protein levels (A-B). Treatment of hPCLS with OCA, Rifampicin or CITCO did not induce the mRNA expression of FOXM1B, CCND1 and PCNA (C). Four slices were included per condition, the experiment was replicated three times using slices derived from different patients. A representative experiment is depicted. Differences among four groups were analyzed with Kruskal-Wallis test, with Dunn's multiple comparison test to compare differences between control and specific treatment. FGF19 levels between control and OCA group was compared with the Mann-Whitney $U$ test. Data is depicted as median [IQR]. Asterisks indicate significance levels: ${ }^{*} \mathrm{P}<0.05,{ }^{*} \mathrm{P}<0.01$. 


\section{Discussion}

In the current study, responsiveness of hPCLS to FGF19 could not be verified at the level of ERK1/2, a kinase that is activated following binding of FGF19 to its receptor FGFR4/KLB. ERK1/2 activation, assessed by detection of its phosphorylated form, was already maximal without addition of FGF19. This likely results from external triggers during harvesting and/or processing of the liver tissue for hPCLS preparation. At the time, we did not investigate whether 'spontaneous' activation of ERK was sustained until the time (18-22 hrs) of harvesting of PCLS for RT-qPCR analysis, and if this could provide an explanation for lack of FGF19 responsiveness and low/undetectable expression of known FGF19 target genes. Expression of FGF19 receptors FGFR4 and KLB was somewhat lowered during hPCLS culturing, but remaining expression after $>18 \mathrm{hrs}$ was deemed high enough to allow FGF19 signaling. Unfortunately, the expression of FGF19-regulated genes could not be used as a readout of FGF19 activity, as CYP7A1 was undetectable after $>19 \mathrm{hrs}$ of culturing and PCK1 was drastically reduced. Although employed recombinant FGF19 was biologically active at the tested dose $(100 \mathrm{ng} / \mathrm{mL})$ in an unrelated experimental setting (e.g. HepG2 cells), we could not verify FGF19 responsiveness in hPCLS. Hence, we cannot draw a conclusion with regard to involvement of a FGF19/IL6 axis in driving hepatocellular proliferation in a human model system.

In our study, we found marked increase of IL6 mRNA expression and protein levels (in medium) in LPS-treated hPCLS. This may be because LPS induces innate immune response through activation of TLR4 in Kupffer cells. This is consistent with Wu et al 14 , who report that hPCLS can be a valuable model to study human innate immunity. Despite marked increase of IL6 protein in the medium, the cell proliferation (CCND1, PCNA) and inhibition of apoptosis (BIRC5) related genes remained unchanged with LPS treatment. An explanation may be that absolute IL6 levels were not high enough for efficient activation of STAT3. Measuring STAT3 phosphorylation or direct testing of the effect of exogenous IL6 appeared useful to verify suitability of hPCLS to study liver regeneration.

In its current form, the hPCLS model is not suitable for studying FGF19 actions, and improvements to maintain a fully differentiated state of hepatocytes seems pivotal. A combination of five small molecules, $5 \mathrm{C}$, synergistically regulated multiple signaling pathways to maintain global gene expression pattern of human functional hepatocytes 
over 4 weeks in vitro (2D culture) ${ }^{23}$. We used the same $5 \mathrm{C}$ condition to culture hPCLS. However, $5 \mathrm{C}$ treatment failed to prevent the general decrease of FXR/FGF19 pathway related genes in our study, including expression of NTCP that was maintained for 4 weeks in isolated hepatocytes ${ }^{23}$. Similar to $5 \mathrm{C}$, Geltrex couldnot maintain expression of genes related to the FXR/FGF19 pathway. The absence of effectiveness of $5 \mathrm{C}$ in hPCLS model may be due to the differences between 2D and 3D culture system. Optimized concentrations of the five chemicals and culture time can be further investigated for hPCLS culture. In addition, as the targets of $5 \mathrm{C}$ components were not tested, the activity of $5 \mathrm{C}$ was undetermined.

Our current study has limitations. Ideally, 'healthy' liver tissue should be used to prepare hPCLS. The pathological state (e.g. chemotherapy-associated liver injury) of the liver tissue was not assessed before the preparation of PCLS. Full evaluation of histological status of liver tissue may be needed prior to PCLS culture. In addition, as loss of a differentiated hepatocyte phenotype is observed during PCLS culture, as well as in isolated hepatocytes, culture conditions should be optimized to prevent dedifferentiation. Furthermore, perfusion of PCLS in a microfluidic system may be a promising strategy, which allows a constant delivery of nutrients and oxygen and a continuous removal of waste products ${ }^{25} .25$

CAR activation by CITCO was reported to induce hepatocyte proliferation in PCLS, including slices prepared from the liver of a patient ${ }^{2}$. However, despite carefully replicating experimental conditions we could not reproduce the proliferative effect of CITCO in hPCLS from three distinct patients. Since induction of CAR target gene CYP2E1 by CITCO was not observed in hPCLS, we cannot draw a conclusion on CAR action.

In summary, while treatment with LPS, OCA or rifampicin elicited clear transcriptional responses in hPCLS, indicative for overall cellular viability and biological responsiveness, efficacy of FGF19 and CAR treatment could not be ascertained in these exploratory experiments. Hence, questions regarding the involvement of the FGF19/IL6 axis in hepatocellular proliferation, and augmentation of liver growth by CAR agonism could not be answered. Systematic evaluation of patient factors (background liver disease, prior chemotherapy, ischemic time, etc), and culturing conditions (e.g. optimized recovery time, strategies to prevent dedifferentiation etc.) should be considered in optimizing the hPCLS as model to study -and therapeutically 
modulate- liver regeneration.

\section{Acknowledgements}

We are indebted to patients for their participation in this study, and are grateful to the HPB surgical team at Maastricht University Medical Center for taking surgical specimens. 


\section{References}

1. Ikegami T, Onda S, Furukawa K, Haruki K, Shirai Y, Gocho T. Small-for-size graft, small-for-size syndrome and inflow modulation in living donor liver transplantation. J Hepatobiliary Pancreat Sci. 2020;27:799-809.

2. Tschuor C, Kachaylo E, Limani P, Raptis DA, Linecker M, Tian Y, et al. Constitutive androstane receptor (Car)-driven regeneration protects liver from failure following tissue loss. $\mathrm{J}$ Hepatol. 2016;65:66-74.

3. Forbes SJ, Newsome PN. Liver regeneration - mechanisms and models to clinical application. Nat Rev Gastroenterol Hepatol. 2016;13:473-85.

4. Khan AS, Garcia-Aroz S, Ansari MA, Atiq SM, Senter-Zapata M, Fowler K, et al. Assessment and optimization of liver volume before major hepatic resection: Current guidelines and a narrative review. Int J Surg. 2018;52:74-81.

5. Schaap FG, Trauner M, Jansen PL. Bile acid receptors as targets for drug development. Nat Rev Gastroenterol Hepatol. 2014;11:55-67.

6. Huang W, Ma K, Zhang J, Qatanani M, Cuvillier J, Liu J, et al. Nuclear receptor-dependent bile acid signaling is required for normal liver regeneration. Science. 2006;312:233-6.

7. Uriarte I, Fernandez-Barrena MG, Monte MJ, Latasa MU, Chang HC, Carotti S, et al. Identification of fibroblast growth factor 15 as a novel mediator of liver regeneration and its application in the prevention of post-resection liver failure in mice. Gut. 2013;62:899-910.

8. Garbers C, Hermanns HM, Schaper F, Müller-Newen G, Grötzinger J, Rose-John S, et al. Plasticity and cross-talk of interleukin 6-type cytokines. Cytokine Growth Factor Rev. 2012;23:8597.

9. Zhou M, Yang H, Learned RM, Tian H, Ling L. Non-cell-autonomous activation of IL-6/STAT3 signaling mediates FGF19-driven hepatocarcinogenesis. Nat Commun. 2017;8:15433.

10. Jiang Y, Que W, Zhu P, Li XK. The Role of Diverse Liver Cells in Liver Transplantation Tolerance. Front Immunol. 2020;11:1203.

11. Ding BS, Cao Z, Lis R, Nolan DJ, Guo P, Simons M, et al. Divergent angiocrine signals from vascular niche balance liver regeneration and fibrosis. Nature. 2014;505:97-102.

12. Modares NF, Polz R, Haghighi F, Lamertz L, Behnke K, Zhuang Y, et al. IL-6 trans-signaling controls liver regeneration after partial hepatectomy. Hepatology. 2019.

13. Ding BS, Nolan DJ, Butler JM, James D, Babazadeh AO, Rosenwaks Z, et al. Inductive angiocrine signals from sinusoidal endothelium are required for liver regeneration. Nature. 2010;468:310-5.

14. Wu X, Roberto JB, Knupp A, Kenerson HL, Truong CD, Yuen SY, et al. Precision-cut human liver slice cultures as an immunological platform. J Immunol Methods. 2018;455:71-9.

15. de Graaf IA, Olinga P, de Jager MH, Merema MT, de Kanter R, van de Kerkhof EG, et al. Preparation and incubation of precision-cut liver and intestinal slices for application in drug metabolism and toxicity studies. Nat Protoc. 2010;5:1540-51.

16. Wu W, Wu Q, Liu X. Chronic activation of FXR induced liver growth with tissue-specific targeting Cyclin D1. Cell Cycle. 2019.

17. Starokozhko V, Abza GB, Maessen HC, Merema MT, Kuper F, Groothuis GM. Viability, function and morphological integrity of precision-cut liver slices during prolonged incubation: Effects of culture medium. Toxicol In Vitro. 2015;30:288-99.

18. Ruijter JM, Ramakers C, Hoogaars WM, Karlen Y, Bakker O, van den Hoff MJ, et al. Amplification 
efficiency: linking baseline and bias in the analysis of quantitative PCR data. Nucleic Acids Res. 2009;37:e45.

19. Dentener MA, Bazil V, Von Asmuth EJ, Ceska M, Buurman WA. Involvement of CD14 in lipopolysaccharide-induced tumor necrosis factor-alpha, IL-6 and IL-8 release by human monocytes and alveolar macrophages. J Immunol. 1993;150:2885-91.

20. Schaap FG, van der Gaag NA, Gouma DJ, Jansen PL. High expression of the bile salthomeostatic hormone fibroblast growth factor 19 in the liver of patients with extrahepatic cholestasis. Hepatology. 2009;49:1228-35.

21. Lauschke VM, Vorrink SU, Moro SM, Rezayee F, Nordling A, Hendriks DF, et al. Massive rearrangements of cellular MicroRNA signatures are key drivers of hepatocyte dedifferentiation. Hepatology. 2016;64:1743-56.

22. Vinken M, Vanhaecke T, Rogiers V. Primary hepatocyte cultures as in vitro tools for toxicity testing: quo vadis? Toxicol In Vitro. 2012;26:541-4.

23. Xiang C, Du Y, Meng G, Soon Yi L, Sun S, Song N, et al. Long-term functional maintenance of primary human hepatocytes in vitro. Science. 2019;364:399-402.

24. Lancett P, Williamson B, Barton P, Riley RJ. Development and Characterization of a Human Hepatocyte Low Intrinsic Clearance Assay for Use in Drug Discovery. Drug Metab Dispos. 2018;46:1169-78.

25. van Midwoud PM, Groothuis GM, Merema MT, Verpoorte E. Microfluidic biochip for the perifusion of precision-cut rat liver slices for metabolism and toxicology studies. Biotechnol Bioeng. 2010;105:184-94. 



\title{
Chapter 4
}

\author{
Obeticholic acid accelerates portal vein \\ embolization-induced liver hypertrophy by \\ improving bile salt homeostasis
}

Xinwei Chang, Cathy van Himbeeck, Pim B. Olthof, Han Jin, Zita Soons, Floor Huisman, Rowan van Goolen, Peter L. Jansen, Thomas van Gulik, Michal Heger, Steven W.M. Olde Damink, Frank G. Schaap 


\section{Abstract}

Background: Farnesoid X receptor (FXR) agonist obeticholic acid (OCA) accelerated liver growth following portal vein embolization (PVE) in rabbits. The aim of this study was to study the underlying mechanisms.

Methods: Materials were analyzed from rabbits treated by oral gavage with OCA (10 $\mathrm{mg} / \mathrm{kg}, \mathrm{n}=11$ ) or vehicle (control group, $\mathrm{n}=11$ ) prior to, and during the course of, PVE. Serum total bile salts (TBS), bile acid composition, C4 (marker of bile acid synthesis), and liver synthetic function were determined before, after $3 \mathrm{hrs}$, and at day 1, day 3 , and day 7 after PVE. Ileal and hepatic genes expression were analyzed through targeted and untargeted approaches at day three after PVE, when the growthenhancing effect of OCA was maximal. For correlation analyses, volumes of (non)embolized liver segments, and \%volume gain, were previously determined by CT scan.

Results: We previously reported that PVE induced a larger volume gain of the nonembolized liver lobe in animals treated with OCA. Effects of OCA were largest at 3 days after PVE (volume gain: $56.1 \%$ vs. $26.1 \%$ in OCA- and vehicle-treated animals, respectively). OCA treatment led to earlier normalization of serum TBS levels and lower bile salt content of the non-embolized liver lobe after PVE. OCA decreased mRNA expression of Cyp7a1 (involved in bile salt synthesis) and promoted expression of S/c51a/b (involved in bile salt export) in the non-embolized liver lobe, likely contributing to the observed improved serum and liver bile salt homeostasis. Levels of lithoholic acid (LCA), a hepatotoxic bile salt, were lower in the OCA-treated animals compared to the control animals at day 1 and day $3(P=0.002$ and $P=0.031$, respectively). LCA levels were strongly related with caudal (i.e. non-embolized) liver volume (CLV) increase and the numbers of $\mathrm{Ki}-67$ positive hepatocytes in the nonembolized lobe $(\rho=-0.726, \mathrm{P}<0.001 ; \rho=-0.718, \mathrm{P}=0.009$, respectively). Both targeted and untargeted transcript analysis revealed associations with genes engaged in bile salt homeostasis and liver growth, adding to the notion that OCA-mediated improvement of bile salt homeostasis was in part responsible for enhanced PVEinduced liver growth. Additional factors candidate for mediating growth-stimulating effects of OCA include the secreted factors Bmp3 and Ihh.

Conclusions: OCA improved bile salt homeostasis, which partly contributed to augmented hypertrophy of the non-embolized liver lobe in rabbits. OCA may be 
promising to increase the efficacy of PVE in patients with small future liver remnant planned for (extended) partial liver resection. 


\section{Introduction}

Partial hepatectomy $(\mathrm{PH})$ presents the only curative treatment for malignant hepatobiliary tumors. The robust regenerative capacity of the liver allows up to $70 \%$ resection of liver mass ${ }^{1}$. However, post-hepatectomy liver failure (PHLF) is a major concern and the main cause of postoperative morbidity and mortality ${ }^{2}$. A small future liver remnant (FLR) volume is associated with higher risk of PHLF and postoperative mortality ${ }^{3}$. Portal vein embolization (PVE) is the gold standard procedure to induce hypertrophy of the FLR if volume is deemed insufficient for direct resection, increasing resectability and safety of liver resection at a later stage ${ }^{4}$. However, relatively long waiting time (around 3 to 4 weeks) between PVE and resection puts patients at risk of tumor progression ${ }^{5}$. Pharmacological strategies to accelerate PVE-induced liver growth are lacking in clinical practice.

Bile salt signaling is involved in liver regeneration triggered by $\mathrm{PH}$, as well as in liver growth in the absence of a surgical stimulus viz. bile salt feeding ${ }^{6}$. The nuclear bile salt receptor farnesoid $X$ receptor (FXR) and FXR-regulated mediators (e.g. fibroblast growth factor 19, FGF19, termed Fgf15 in rodents), are key players in these processes. Mice deficient in either Fxr or Fgf15 have impaired liver regeneration and high mortality rates following $\mathrm{PH}$ 6, 7. Fxr and FGF19/Fgf15 are essential to maintain bile salt homeostasis, which is achieved by (transcriptional) regulation of hepatic synthesis and import/export of bile salts. Dysregulation of bile salt homeostasis, resulting in hepatic bile salt accumulation and attendant cytotoxicity, as well as diminished action of bile salt-regulated mitogens, underlies defective regeneration and mortality in these mice strains. In patients undergoing $\mathrm{PH}$, serum bile salt levels are positively associated with liver regeneration ${ }^{8-10}$. Above findings highlight the importance of maintained bile salt homeostasis in the remnant liver, thus balancing beneficial signaling actions and detrimental consequences of bile salt overload.

In patients undergoing PVE, we observed that serum bile salt levels after PVE were early predictors of post-PVE liver volume and function ${ }^{9}$. This suggested that transient elevation of serum bile salts after PVE, provides a signal to increase liver mass. To directly test whether bile salt/FXR signaling was involved in PVE-induced liver growth, we previously examined the effects of obeticholic acid (OCA) in a rabbit model of PVE 11. OCA is a first-in-class selective and potent agonist of FXR, which is currently evaluated in clinical trials in patients with cholestatic or metabolic liver disease ${ }^{12-14}$. In 
our experimental model, we noted that OCA accelerated PVE-induced growth of the non-embolized liver segment, with the largest effect seen in the initial three days after PVE. Augmented volume gain was due to enhanced proliferation of hepatocytes, and was accompanied by a functional increase in secretory function. In the current study, we sought to determine the molecular pathways beyond the hyperplastic action of OCA in this model. To this end, we tested the hypothesis that improved bile salt homeostasis is involved. Furthermore, molecular players were examined by targeted and untargeted transcript analysis.

\section{Methods}

\section{Animal specimens}

For the current study we analyzed plasma samples and tissue specimens that were collected at the time of the original study ${ }^{11}$. In brief, rabbits ( $n=12$ per group, note that in each group, one animal had to be euthanized prior to scheduled sacrifice) were pretreated for one week (day -7 and day -1 ) by daily gavage of OCA $(10 \mathrm{mg} / \mathrm{kg}$ ) or vehicle, before undergoing embolization of the three cranial lobes (Supplementary Fig. 1). The respective treatments were continued after PVE, with blood samples taken after $3 \mathrm{hrs}$, day 1 , day 3 , and day 7 . Animals were sacrificed after day 3 ( $n=6$ per group) or day 7 ( $n=5$ per group), and terminal ileum, caudal (non-embolized) and cranial (embolized) liver lobes were harvested, snap-frozen and stored at $-80^{\circ} \mathrm{C}$. For correlation analyses, volumes of (non)embolized liver segments, and \%volume gain, were previously determined by CT scan.

\section{Quantification of bile salts}

Bile salts were extracted from homogenates of (non)embolized liver segments in $75 \%$ ethanol as described ${ }^{15}$. Total bile salt (TBS) levels in serum and liver extracts were determined through an enzymatic cycling method according to the manufacturer's protocol (Diazyme Laboratories, CA, USA). Liver bile salts were normalized to wet weight of liver specimens. Serum bile salt composition was determined by LC-MS as will be detailed elsewhere (van Eijk, Lenicek, Olde Damink and Schaap, manuscript in preparation). Serum 7-a-hydroxy-4-cholesten-3-one (C4, a serum marker of bile salt synthesis) was assessed simultaneously. For calculation of fractional bile salt composition (Fig. 3A), unconjugated (e.g. DCA) and conjugated forms (e.g. GDCA and 
Chapter 4

TDCA) of a particular species were summed.

\section{Targeted transcript analyses}

cDNA synthesis and targeted analysis of expression levels by RT-qPCR was previously described ${ }^{11}$. Heretofore unreported transcripts were analyzed for the present study. Employed primer sequences are provided in Supplementary Table S1.

\section{RNA sequencing}

Total RNA was extracted from (non)embolized liver lobes and terminal ileum tissue from animals sacrificed at day 3, with the RNeasy Plus Mini Kit (Qiagen, Hilden, Germany). RNA concentration and quality were determined on a 2100 Bioanalyzer (Agilent, CA, US). All samples for RNA sequencing had a RNA integrity number (RIN) of 7.1 or higher. cDNA library preparation, next-generation sequencing (Illumina sequencing platform, paired-end reads of $150 \mathrm{bp},>30$ million reads per sample), and mapping to the rabbit genome, were conducted by Eurofins Genomics (Ebersberg, Germany). Rabbit genes were converted into human orthologues using biomaRt $R$ package (v2.48.1) based on Ensembl gene ID. Gene wise read counts were collected from the samples to be compared, and genes receiving less than 10 reads on average across all the samples, were removed. Raw gene counts were normalized separately for ileal and hepatic samples using R package DESeq2 (v1.32.0) ${ }^{16}$. A fold change of $\geq 2$ in either direction, an average normalized gene count $>100$, and a BenjaminiHochberg false discovery rate (FDR) corrected $p<0.10$ was used for identifying differentially expressed genes (DEGs) using DESeq2 (v1.32.0) with default settings (independent filtering $=$ TRUE, alpha $=0.10$ ). Pathway analysis was performed using Metascape program ${ }^{17}$.

\section{ELISA}

Plasma levels of coagulation factor II, a measure of liver synthetic function, was determined by ELISA per manufacturers protocol (cat.nr abx053007, Abbexa, Cambridge, UK).

\section{Statistical analysis}

Data are presented as median [interquartile range] or mean \pm standard error of mean (SEM) when appropriate. The mean of day -7 and day -1 blood measurements was taken as baseline value. Repeated measures analysis of variance (ANOVA) or a mixed 
effect model (for blood parameters from baseline to day 7) was used to evaluate longitudinal changes, with Dunn's multiple comparison test to compare differences between baseline and subsequent individual time points. Differences between OCAtreated and control animals were analyzed with the Mann-Whitney $U$ test. Correlations were assessed by Spearman's correlation coefficient $(\rho)$. The correlation coefficients between liver growth and differentially expressed genes that were identified in all changed pathways were corrected with Benjamini-Hochberg false discovery rate (FDR) method. $P$ values $<0.05$ were considered statistically significant. Statistical analyses were performed using GraphPad Prism 8.0 (GraphPad Software Inc., CA, USA), SPSS 24.0 (IBM SPSS Inc, Chicago, Illinois, USA) and R Statistical Software 4.0.5 (R Foundation for Statistical Computing).

\section{Results}

We previously reported that PVE induced a larger volume gain of the non-embolized liver lobe in animals treated with OCA ${ }^{11}$. As effects of OCA were largest at 3 days after PVE (volume gain: $56.1 \%$ vs. $26.1 \%$ in OCA- and vehicle-treated animals, respectively), attempts to find a molecular explanation focused on this time point. We hypothesized that improved bile salt homeostasis contributes to augmented liver volume gain in OCA-treated animals.

\section{Improved bile salt homeostasis in OCA-treated animals}

First, we assessed serum TBS levels during the course of PVE. Bile salt levels did not alter during the pre-treatment phase, and were not affected by OCA treatment (data not shown). PVE induced a rapid elevation of serum TBS, with peak levels on average 8.2-9.3 fold higher than baseline values (Fig. 1A). This increase likely reflects limited spare capacity of the non-embolized lobe (ca. $24 \%$ of total liver volume) to extract bile salts from the portal circulation, resulting in their spill-over in the systemic circulation. Maximum elevation and time to reach this peak level were different between groups. TBS levels peaked at day 1 in controls, but already after $3 \mathrm{~h}$ in OCA-treated animals $(P=0.012)$. The trend in time tended to be different between experimental groups $\left(P_{\text {time }}{ }^{*}\right.$ roup $\left.=0.063\right)$, as reflected by lower TBS levels in the OCA group at day $1(P=0.023)$ and apparent normalization of serum levels after day 3 (vs. day 7 in controls) (Fig. 1AB). 
Next, we examined hepatic bile salt content at 3 days after PVE. In the non-embolized (hyperplastic) lobe, bile salts levels were 1.7 fold lower upon OCA treatment (67 [60.377.4] vs. 110.6 [88.6-169.6] nmol/g liver; $P=0.015)$ (Fig. 1C). In line with abrogated portal supply, bile salt content of the embolized lobes was lower relative to the nonembolized lobe $(P=0.002)$, with a tendency $(P=0.055)$ to further lowering by OCA (Fig. 1C). Hence, both circulating and hepatic bile salt levels were reduced in OCA-treated animals, indicating improved bile salt homeostasis.
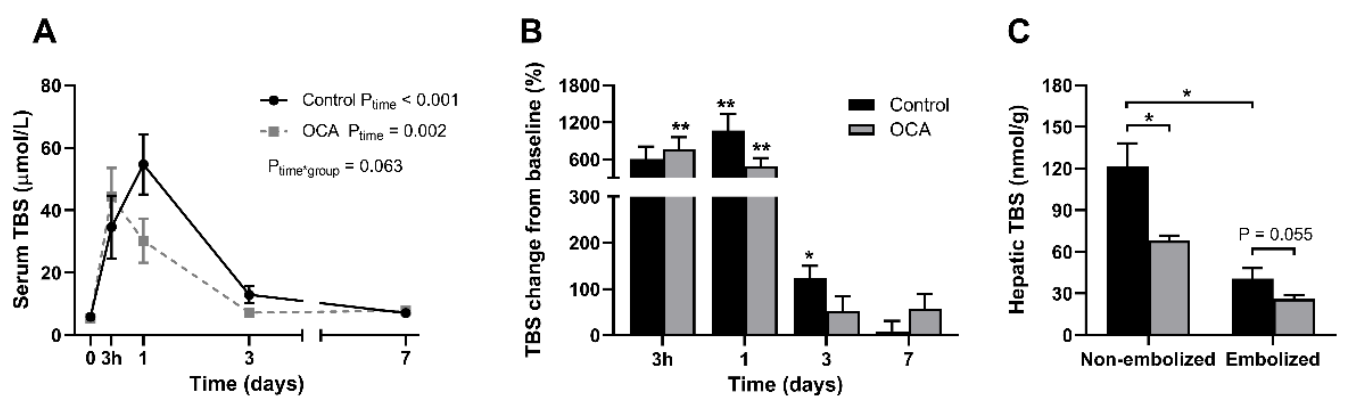

Figure 1. Improved bile salt homeostasis in OCA-treated animals. (A) Serum TBS levels peaked earlier in OCA-treated animals ( $n=11$ per group). (B) Serum TBS levels normalized faster in OCA-treated animals. (C) Non-embolized hepatic lobe TBS levels at day 3 were lower in OCA-treated animals than controls, and TBS levels were lower in embolized than nonembolized lobes within control animals ( $n=6$ per group). Longitudinal changes were evaluated by mixed effect model. Differences between OCA-treated animals and control animals were analyzed with the Mann-Whitney $U$ test. Differences between embolized and non-embolized hepatic lobes within control animals were compared with the Wilcoxon signed-rank test. Data is depicted as mean \pm SEM. $P$ values are depicted. Asterisks indicate significance levels: ${ }^{*} \mathrm{P}<0.05,{ }^{*} \mathrm{P}<0.01$. TBS, total bile salt.

\section{Regulation of genes involved in bile salt homeostasis by OCA}

To find an explanation for reduced hepatic bile salt content in OCA-treated animals, expression of ileal and hepatic genes engaged in bile salt homeostasis was examined at day 3. Note that expression of some of these genes was previously reported ${ }^{11}$, and this data has been duplicated to allow a comprehensive overview of factors involved in hepatic bile salt homeostasis. At the level of the terminal ileum, OCA target engagement was evident by induction of Fxr target genes Slc51a and Shp (Fig. 2A). Note that we could not detect expression of Fxr target Fgf19 in the terminal ileum. This endocrine factor plays an important role in repression of bile salt synthesis by targeting 
hepatic Cyp7a1 expression. Of note, Cyp7a1 expression in the non-embolized (i.e. caudal) lobe was markedly lower (-6.9 fold, $\mathrm{P}=0.004$ ) in OCA-treated animals (Fig. 2B). Fgf19 may be involved in this repression by binding to its hepatic receptor composed of Fgfr4 (maintained expression) and $\beta$-Klotho (slightly reduced expression). Caudal expression of Shp, another Fxr-induced negative regulator of Cyp7a1 expression, was not affected by OCA treatment. Hepatic Fxr targets that were upregulated included Fabp1, a bile salt-binding protein implicated in sequestering potentially harmful hydrophobic bile salts, and bile salt transporter subunits S/c51a/b (Fig. 2B). With maintained expression of bile salt uptake protein Ntcp, reduced bile salt levels in the non-embolized lobe of OCA-treated animals may be explained by reduced bile salt synthesis (Cyp7a1) combined with enhanced basolateral export of bile salts (Slc51a/b). Unexpectedly, the lower expression of Cyp7a1 mRNA in the non-embolized lobe in the OCA group, was not accompanied by reduced levels of $\mathrm{C} 4$ in the systemic circulation at day 3 (Fig. 2C). C4 is a serum marker for bile salt synthesis. Its validity as a biomarker has been examined mainly in rodent models and healthy subjects. Serum C4 levels did not change during the course of PVE in control animals $\left(P_{\text {time }}=0.609\right)$, whereas a transient drop was noted in the OCA-treated animals $\left(P_{\text {time }}=0.013\right)$ (Fig. 2C). The trend in time did not differ between groups $\left(P_{\text {time }}{ }^{*}\right.$ roup $\left.=0.728\right)$. For reasons unknown, a correlation between Cyp7a1 mRNA and serum C4 was not apparent in this study (Fig. 2D).

A

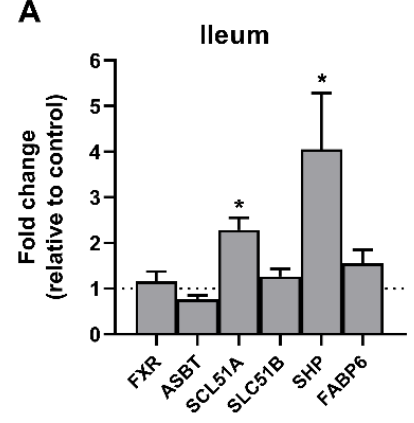

C

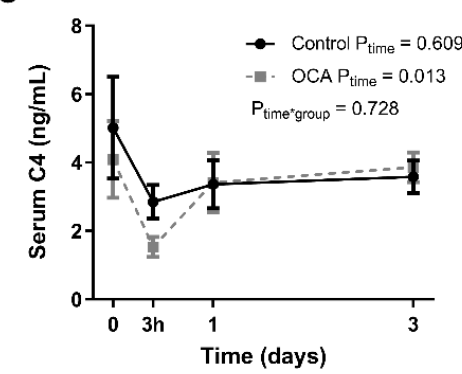

B



D

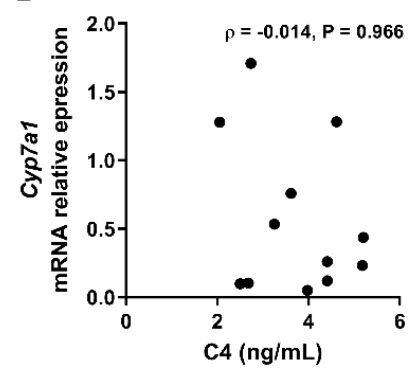

Figure 2. Regulation of genes involved in bile salt homeostasis by OCA. (A) lleal 
expression of Shp and Slc51a were upregulated in OCA-treated animals ( $\mathrm{n}=6$ per group). (B) Expression of Cyp7a1, Cyp8b1 and $\beta$-Klotho were downregulated, and expression of S/c51a/b and Fabp 1 were upregulated in the non-embolized liver lobe in OCA-treated animals $(n=6$ per group). (C) Serum C4 levels showed a transient drop in OCA-treated animals, but not in control animals ( $n=6$ per group). (D) Decreased mRNA expression of Cyp7a1 in the non-embolized liver lobe was not accompanied by reduced levels of C4 in the systemic circulation at day 3 $(n=12)$. Differences between OCA-treated animals and control animals were analyzed with the Mann-Whitney $U$ test. Correlations were evaluated with Spearman correlation coefficient $(\rho)$. Longitudinal changes were evaluated by mixed effect model. Data is depicted as mean \pm SEM. $P$ values are depicted. Asterisks indicate significance levels: ${ }^{*} P<0.05,{ }^{* *} P<0.01$. $C 4,7-\alpha-$ hydroxy-4-cholesten-3-one.

\section{Effect of OCA on serum bile salt composition}

Individual bile salt species differ in their signaling activities and cytotoxic potential. We next evaluated if the composition of systemic bile salts was affected during the course of PVE, and by treatment with OCA. In rabbits, deoxycholic acid (DCA) and conjugated forms thereof, are the dominant bile salt species, in this study representing ca. $65 \%$ of circulating bile salts at baseline (Fig. 3A). Note that OCA and its conjugated forms amounted to ca. $10 \%$ of circulating bile salt species at baseline in OCA-treated animals. With exception of HDCA and OCA in the OCA-treated animals, fractional serum bile salt composition did not show major shifts during the course of PVE (Fig. 3A). Mole fraction of HDCA was lower in the OCA-treated animals than controls after PVE $\left(P_{\text {group }}=0.001\right)$, but the downward trend in time was similar between groups $\left(P_{\text {time }}{ }^{*}\right.$ roup $\left.=0.954\right)$ (Fig. 3B). Interestingly, mole fraction of OCA was highest in the period (i.e. between day 1 and 3 ) where the volume of the non-embolized liver lobe increased most steeply.

Bile salt hydrophobicity index $(\mathrm{HI})$ is a measure associated with bile salt-induced cytotoxicity. We calculated the $\mathrm{HI}$ of serum bile salts using the molar fraction and the previously reported hydrophobicity of individual bile salts ${ }^{18}$. Note that OCA was unknown at the time the $\mathrm{HI}$ concept was proposed. For calculating contributions of OCA (conjugates) we used the mean value reported for chenodeoxycholic acid (CDCA) (conjugates) and lithocholic acid (LCA) (conjugates). Chromatographic behavior of OCA (a.k.a. 6-ethyl-CDCA) on the employed reverse phase column justifies this approach (data not shown). The overall HI of serum bile salts was increased after PVE 
in both control and OCA-treated animals, but the trend in time was similar between groups $\left(P_{\text {time }}{ }^{*}\right.$ group $=0.231$, Fig. $\left.3 \mathrm{C}\right)$. This is indicative for a more cytotoxic composition after PVE.

Next, we investigated the change of absolute levels of bile salt species during the course of PVE. Only species with a significant trend in time and a significant association between levels and liver growth parameters at day 3 , are discussed. Serum levels of LCA, a minor but well-known hepatotoxic bile salt ${ }^{19}$, were transiently elevated after PVE, reaching peak levels that were on average 13 and 10 -fold higher compared to baseline values in control and OCA-treated animals, respectively ( $P<0.001$ and $P=0.002$, respectively) (Fig. 3D). During the course of $P V E$, LCA showed a faster normalization in the OCA-treated animals compared to control animals $\left(P_{\text {time }}{ }^{*}\right.$ group $\left.=0.012\right)$. LCA levels were lower in the OCA-treated animals compared to the controls at day 1 and day $3(P=0.002$ and $P=0.031$, respectively) (Fig. 3D). As mirrored by lower fractional composition, HDCA levels were reduced in OCA-treated animals following PVE $\left(P_{\text {time }}=0.005\right)$, and lower relative to controls from $3 \mathrm{~h}$ onwards ( $P$ values between <0.001 and 0.009) (Fig. 3E) In addition, DCA levels showed a trend towards faster return to baseline values after PVE $\left(P_{\text {time*group }}=0.054\right)$ in the OCA group (Fig. 3F). Longitudinal changes of the other bile salt species were similar in controls and OCAtreated animals or showed no relation with liver growth. (Supplementary Fig. S2,

\section{Supplementary Table 2).}

\section{Associations between bile salt-related parameters and liver growth}

To evaluate the associations between bile salt parameters and liver growth-related parameters, we performed Spearman correlation analyses using day 3 values. Serum TBS levels were negatively correlated $(\rho=-0.518, P=0.014)$ with volume gain of the non-embolized lobe (i.e. CLV) (Fig. 4A). Remarkably, LCA levels were strongly correlated with CLV increase and the numbers of Ki-67 positive $\left(\mathrm{Ki}-67^{+}\right)$hepatocytes in this segment $(\rho=-0.726, P<0.001 ; \rho=-0.718, P=0.009$, respectively) (Fig. 4B-C). Additionally, serum levels of HDCA and DCA were both negatively correlated with CLV increase $(\rho=-0.491, P=0.020 ; \rho=-0.527, P=0.012$, respectively) (Fig. 4D-E). No significant correlations between liver growth parameters and the levels of hepatic TBS or serum C4 were observed. All correlations are summarized in Supplementary Table S2.

LCA induces cholestasis and impairs biliary bile salt secretion in rats ${ }^{20}$. We assessed 


the relations between LCA levels and genes involved in bile salt homeostasis. Serum LCA levels were positively correlated with the expression of Cyp7a1, and negatively associated with expression of S/c51a in the non-embolized liver lobe $(\rho=+0.585, P=$ 0.046; $\rho=-0.706, P=0.015$, respectively) (Fig. 4F-G).

A

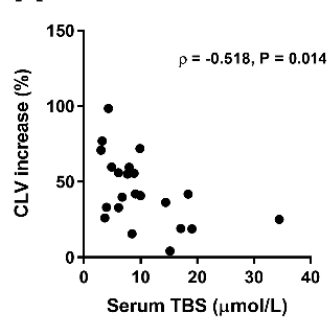

D

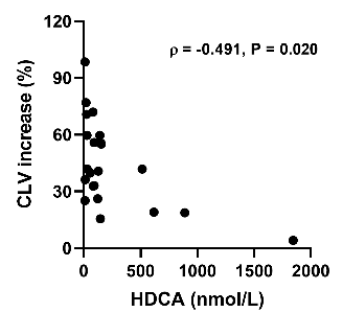

$\mathbf{F}$

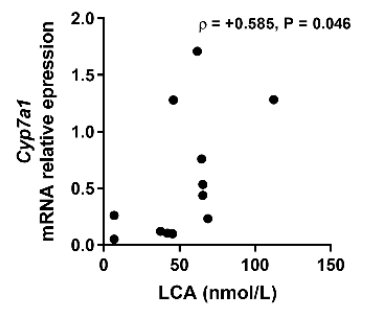

B

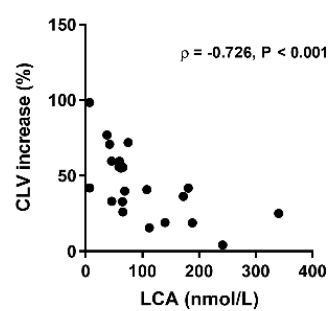

E

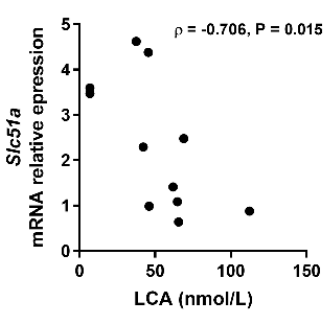

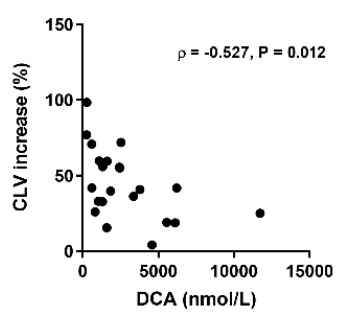

G
C

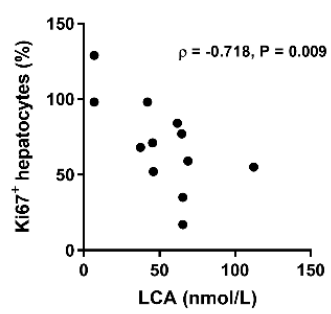

Figure 4. Associations between individual serum bile salt species and liver growth. (A) Serum TBS levels were negatively correlated with CLV increase. Serum LCA levels were negatively correlated with CLV increase (B) and the numbers of Ki-67 positive hepatocytes in this segment (C). Serum (D) HDCA and (E) DCA levels were negatively correlated with CLV increase. Serum LCA levels were positively correlated with the expression of Cyp7a1 (F) and negatively correlated with the expression of S/c51a in the non-embolized liver lobe (G). Correlations were evaluated with Spearman correlation coefficient $(\rho)$. P values are depicted. TBS, total bile salt; CLV, caudal liver volume (non-embolized liver volume); LCA, lithocholic acid; HDCA, hyodeoxycholic acid.

\section{OCA does not affect liver synthetic function}

We previously reported that OCA enhanced liver transport function at day 3 after PVE, as assessed by uptake of mebrofenin ${ }^{11}$. To assess whether OCA treatment affects the 
synthetic function of the liver as well, plasma coagulation factor II levels were determined. Factor II levels did not change after PVE in either group (Supplementary Fig. S3).

\section{Analyses of genes involved in cell cycle regulation}

Apart from bile salt homeostatic actions, Fxr has been implicated in regulation of cell proliferation, including direct upregulation of Foxm $1 \mathrm{~b}$, which is essential for cell cycle re-entry of (quiescent) hepatocytes after $\mathrm{PH}^{6}$. Foxm $1 \mathrm{~b}$ expression was notably higher in the non-embolized liver lobe, but no further modulation by OCA was observed (Fig. 5A). However, Foxm1b target gene Cdc25b was induced by OCA specifically in the non-embolized lobe ( $P=0.006)$ (Fig. 5B). Gene expression of the proliferation marker Mki67 and proliferating cell nuclear antigen (Pcna) was not affected by OCA treatment (Supplementary Fig. S4). Cdc25b expression was negatively correlated with expression of Cyp7a1 $(\rho=-0.601, P=0.039)$, and positively correlated with expression of Fxr targets S/c51a $(\rho=+0.745, P=0.008)$ and S/c51b $(\rho=+0.804, P=0.002)$, the number of $\mathrm{Ki}-67^{+}$hepatocytes $(\rho=+0.701, \mathrm{P}=0.011)$ and the volume gain of nonembolized liver lobe $(\rho=+0.566, P=0.055)$ (Fig. 5C-G).

\section{RNA sequencing}

For systematic identification of FXR-regulated genes that may underlie the augmented volume gain of the non-embolized liver lobe, we conducted RNAseq analysis of terminal ileum, and embolized and non-embolized liver lobes harvested at day 3 after PVE. On average around 33 (range 30-74) million reads were produced per sample. Approximately $74 \%$ of the reads could be mapped to the rabbit genome.

\section{OCA-induced changes in the terminal ileum}

Ileum-derived FGF19 regulates hepatic bile salt synthesis and may act as a direct mitogen for hepatocytes ${ }^{7}$. Ileal factors are potentially involved in OCA-augmented liver growth and were studied by differential gene expression analysis and correlative analysis. Note that textual description of results from correlation analysis focuses on secreted signaling factors for which autocrine, endocrine or paracrine modulation of cell growth is easy to conceptualize.

OCA treatment resulted in 67 differentially expressed genes (DEGs, 34 up and 33 down) in the terminal ileum (Fig. 6A). FXR target Shp/NrOb2 is one of the most highly 
A

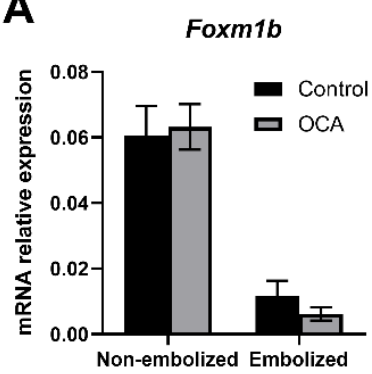

C

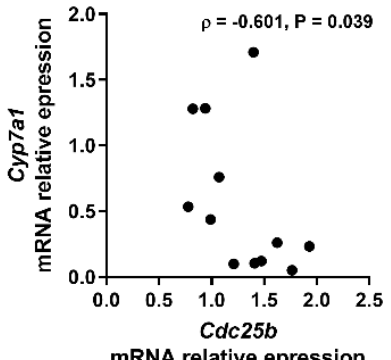

$\mathbf{F}$

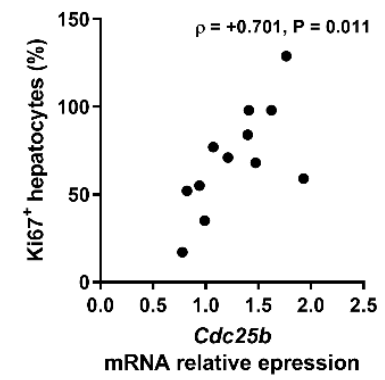

B

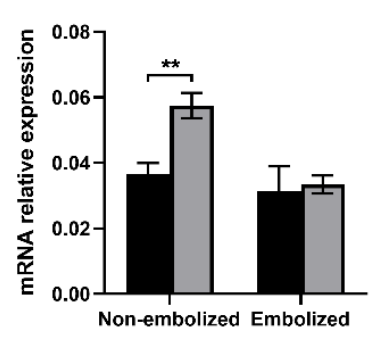

D

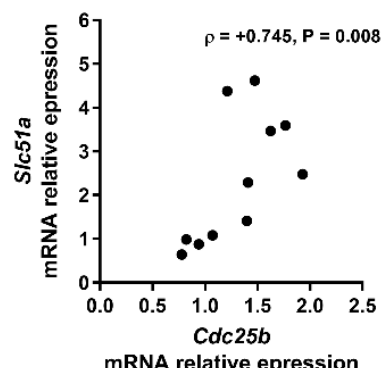

G

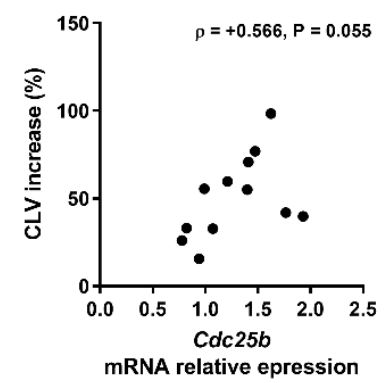

E

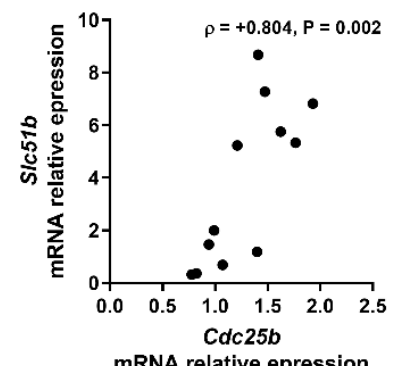

Figure 5. Analyses of genes involved in cell cycle regulation. OCA did not affect mRNA expression of (A) Foxm1b, whereas induced the mRNA expression of (B) Cdc25b. Cdc25b expression was negatively correlated with expression of (C) Cyp7a1, and positively correlated with the expression of Fxr targets (D) S/c51a and (E) S/c51b, (F) the number of Ki-67 positive hepatocytes and (G) the volume gain of non-embolized liver lobe (i.e. CLV). Differences between OCA-treated animals and control animals were analyzed with the Mann-Whitney $U$ test. Correlations were evaluated with Spearman correlation coefficient $(\rho)$. P values are depicted. Asterisks indicate significance levels: ${ }^{* *} \mathrm{P}<0.01$.

upregulated genes, which is consistent with RT-qPCR results. Additionally, OCA induced expression of secreted factors C1qa, C1qb and C1qc, which are components of the complement system that participates in liver regeneration ${ }^{21}$. The top 10 of induced and repressed genes can be found in Supplementary Table 3. We performed pathway enrichment analysis using all DEGs. The top 3 of ileal pathways affected by 
OCA were 'C1q complex', 'regeneration', and 'cell-substrate adhesion' (Fig. 6B). Genes in the affected pathways are presented in Supplementary Table 4. We used Spearman correlation coefficient to evaluate associations between liver growth and DEGs identified in the changed pathways. Significant associations are marked in the heatmap (Fig. 6C). CLV increase was negatively correlated with the expression of the signaling factor bone morphogenetic protein $3(B m p 3)(\rho=-0.601, P=0.039)$, with OCA repressing Bmp3 expression 2 fold. Bmp signaling participates in adult tissue homeostasis including liver regeneration ${ }^{22}$. The numbers of $\mathrm{Ki}-67^{+}$hepatocytes were positively correlated with the expression of C1qa $(\rho=+0.760, P=0.004)$.

A

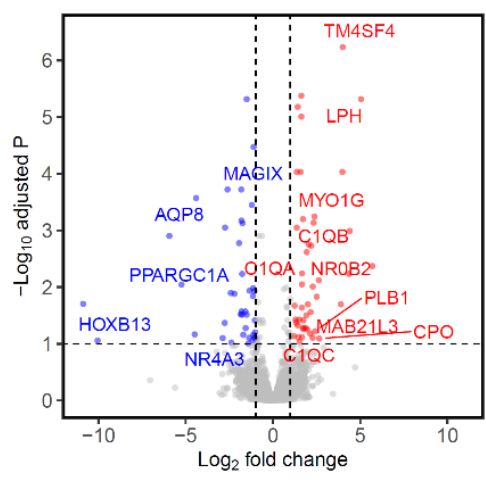

B

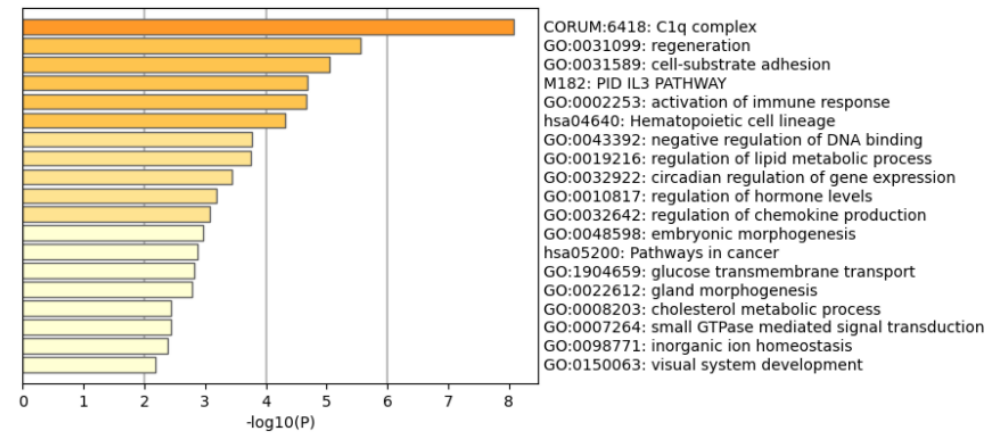

Figure 6. OCA-induced changes in the terminal ileum. (A) Differentially expressed genes induced by OCA in terminal ileum ( $n=6$ per group). (B) Identification of changed pathway by OCA treatment. (C) Heatmaps of Spearman correlation analysis between CLV increase, the number of Ki67 positive hepatocytes and genes present in changed pathways. Asterisks indicate significance levels: ${ }^{*} P<0.05,{ }^{* *} P<0.01$. N.S., not significant. $C L V$, caudal liver volume (i.e. non-embolized liver volume). 


\section{PVE-induced changes in the control liver}

PVE elicited broad transcriptional changes in the liver of vehicle-treated animals, with a total of 467 genes differentially expressed in atrophic and hypertrophic lobes (261 up and 206 down in the non-embolized liver lobe) (Fig. 7A). S/c51b and cell cycle-related genes, such as Cdk1, Ccnb1, Ccnb2 and Cdca3, were among the most highly induced genes in the proliferating liver segment (Supplementary Table 3). Furthermore, the expression of signaling factor Bmp10 was elevated ( 2 fold) in the non-embolized lobe. BMP10 was previously shown to negatively regulate cell proliferation in multiple tumors 23, 24, but has thus far not been linked with compensatory liver growth. Here, the CLV increase was negatively correlated with the expression of $B m p 10(\rho=-0.829, P=0.042)$. Additionally, connective tissue growth factor (Ctgf) was downregulated 2-fold in the non-embolized lobe. CTGF plays an important role in liver regeneration after partial hepatectomy or ethanol/carbon tetrachloride $\left(\mathrm{CCl}_{4}\right)$-induced liver injury ${ }^{25,26}$. The CLV increase was strongly -but negatively-correlated with the expression of $\operatorname{Ctgf}(\rho=-1.000$, $P<0.001)$. Through pathway enrichment analyses, 'Cell cycle, Mitotic' was identified as the most significantly changed pathway, followed by 'Condensation of Prometaphase Chromosomes' and 'monocarboxylic acid metabolic process' (Fig. 7B, Supplementary Table 4). The numbers of $\mathrm{Ki}-67^{+}$hepatocytes strongly correlated with the expression of Cdk1, Ccnb1 and Ccnb2 $(\rho=+0.829, P=0.042 ; \rho=+0.829, P=0.042$; $\rho=+0.943, P=0.005$, respectively) (Fig. 7C). Note that for reasons of graphical clarity, only significant correlations between DEGs and liver growth are depicted in Fig. 7C, with the full set of correlations depicted in Supplementary Fig. S5.

\section{OCA-induced changes in the non-embolized liver lobe}

OCA treatment resulted in 58 DEGs (33 up and 25 down) in the non-embolized proliferating liver lobe (Fig. 8A). Of note, and in line with RT-qPCR findings (Fig. 2B), FXR targets S/c51b and Cyp7a1 were the most highly upregulated and downregulated genes, respectively, in the non-embolized liver segment (Supplementary Table 3). Moreover, secreted factor apolipoprotein $\mathrm{D}(A P O D)$ expression was increased 2 fold. APOD has been related to cell proliferation in bone metabolism and tumors ${ }^{27,}{ }^{28}$. CLV increase was positively correlated with the expression of $A P O D(\rho=+0.580, P=0.048)$. Pathway enrichment analyses showed that 'steroid metabolic process' was the pathway most significantly affected by OCA treatment (Fig. 8B). Additionally, 'Biological oxidations' and 'positive regulation of steroid biosynthetic process' were 
A

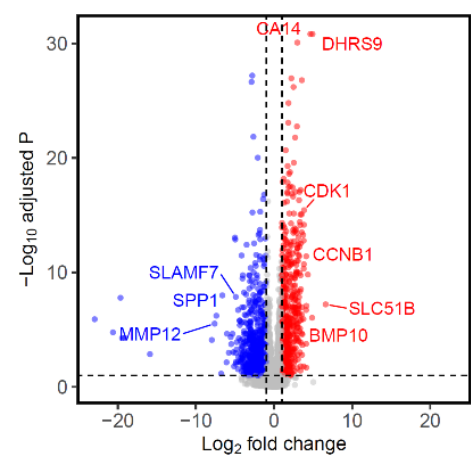

B

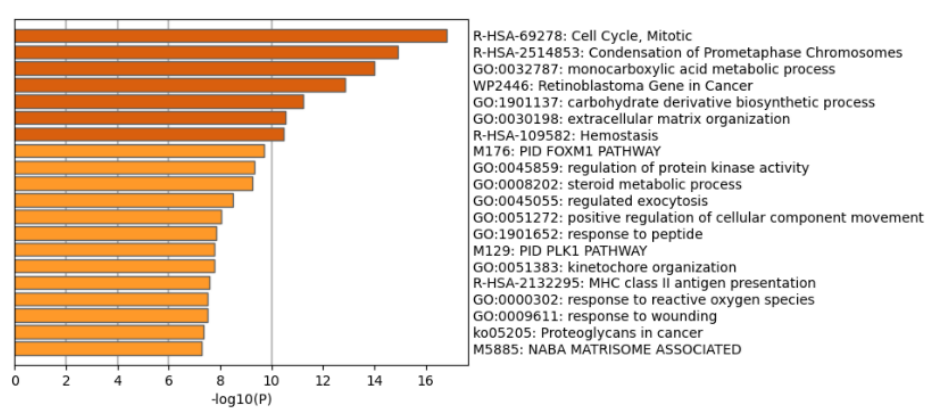

C

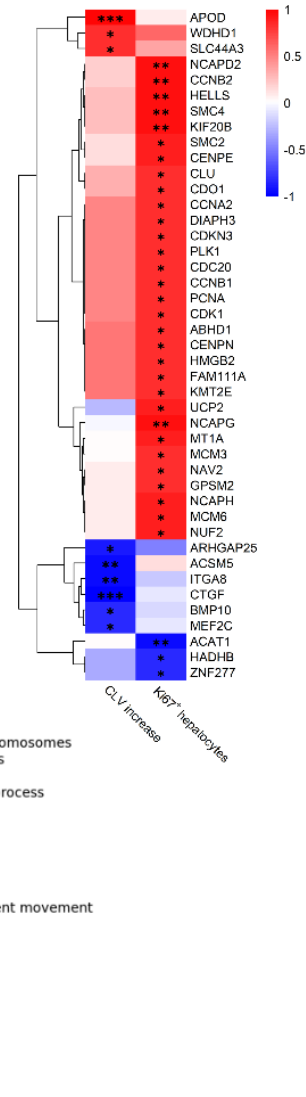

Figure 7. PVE-induced changes in the control liver. (A) Differentially expressed genes induced by PVE in non-embolized and embolized liver lobes ( $n=6$ per group). (B) Identification of changed pathway by PVE. (C) Heatmaps of Spearman correlation analysis between CLV increase, the number of Ki67 positive hepatocytes and genes present in changed pathways. Asterisks indicate significance levels: ${ }^{*} P<0.05,{ }^{* *} P<0.01,{ }^{* * *} P<0.001$. N.S., not significant. CLV, caudal liver volume (i.e. non-embolized liver volume).

among the changed pathways. Remarkably, CLV increase was negatively correlated with the expression of Cyp7a1 and positively with S/c51b expression $(\rho=-0.601$, $P=0.039 ; \rho=+0.629, P=0.029$, respectively) (Fig. 8C). Furthermore, the numbers of Ki$67^{+}$hepatocytes were positively correlated with S/c51b expression and tended to be negatively correlated with Cyp7a1 expression $(\rho=+0.662, P=0.019 ; \rho=-0.539, P=0.070$, respectively). Above correlations suggest that improved bile salt homeostasis directly contributes to OCA-accelerated liver hypertrophy. However, it cannot be excluded that improved bile salt homeostasis is not the cause but the consequence of augmented non-embolized liver volume (and function). Although serum levels of certain bile salt species at day 1 were predictive for non-embolized liver volume at day 3 , further 
empirical examination of above cause-effect relationship is required.

A

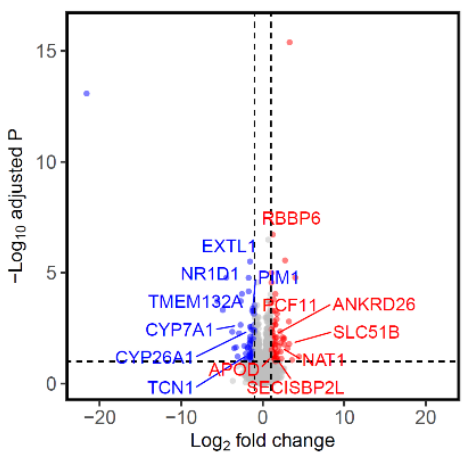

B

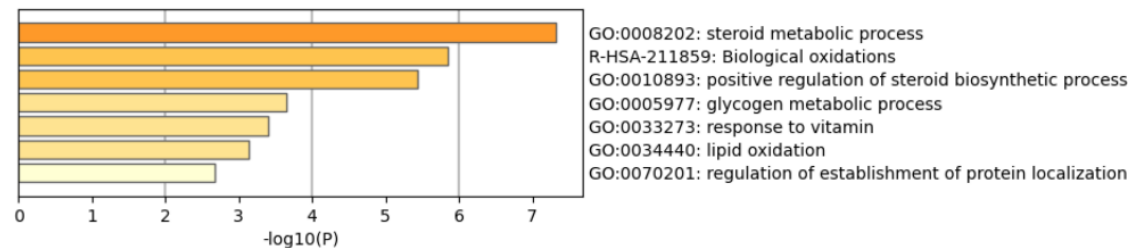

Figure 8. OCA-induced changes in the non-embolized liver lobe. (A) Differentially expressed genes induced by OCA in the non-embolized liver lobe ( $n=6$ per group). (B) Identification of changed pathway by OCA. (C) Heatmaps of Spearman correlation analysis between CLV increase, the number of Ki67 positive hepatocytes and genes present in changed pathways. Asterisks indicate significance levels: ${ }^{*} \mathrm{P}<0.05$, ${ }^{* *} \mathrm{P}<0.01,{ }^{* * *} \mathrm{P}<0.001$. N.S., not significant. CLV, caudal liver volume (i.e. non-embolized liver volume).

\section{OCA-induced changes in the embolized liver lobes}

It is conceivable that signals from the embolized (i.e. atrophic) liver lobes are engaged in compensatory growth of the non-embolized lobe. A total of 63 DEGs (36 up and 27 down) were observed after OCA treatment (Fig. 9A). Cyp7a1 was the most downregulated gene and S/c51b was the most upregulated gene in OCA-treated animals (Supplementary Table 3). In addition, OCA induced expression of Indian hedgehog (Ihh, 2 fold), a secreted signaling factor that is implicated in liver regeneration ${ }^{29}$ and induced following standard $\mathrm{PH}$ as well in experimental two-staged hepatectomy (associating liver partition and portal vein ligation for staged hepatectomy, ALPPS) ${ }^{30}$. The significantly changed pathways in the embolized liver lobes are shown in Fig. 9B and Supplementary Table 4, and include 'steroid metabolic process', 'lipid localization' and 'cellular response to low-density lipoprotein particle stimulus'. CLV 
increase was negatively correlated with the cranial (i.e. embolized lobes) expression of Cyp7a1 $(\rho=-0.608, P=0.036)$. The numbers of $\mathrm{Ki}-67^{+}$hepatocytes in the nonembolized lobe were positively correlated with cranial S/c51b expression, and tended to be positively correlated with $/ h h$ expression $(\rho=+0.715, P=0.009 ; \rho=+0.560, P=0.058$, respectively) (Fig. 9C).

A

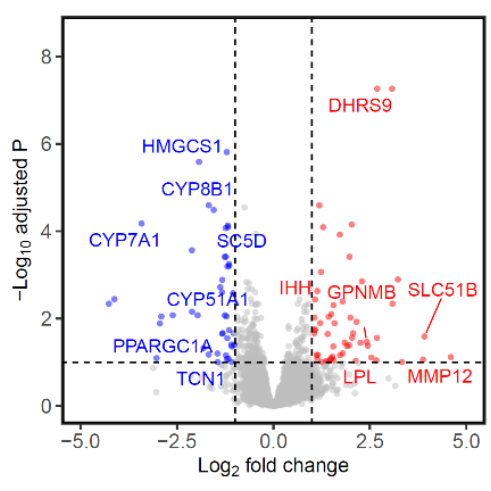

B

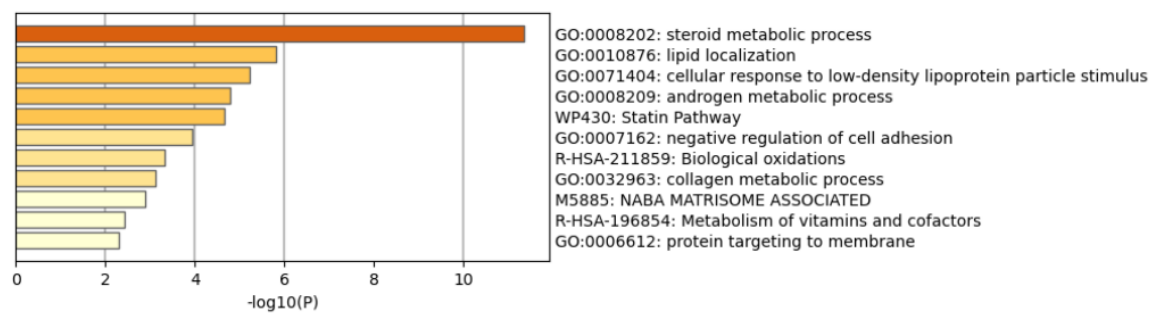

Figure 9. OCA-induced changes in the embolized liver lobes. (A) Differentially expressed genes induced by OCA in the embolized liver lobes ( $n=6$ per group). (B) Identification of changed pathway by OCA. (C) Heatmaps of Spearman correlation analysis between CLV increase, the number of Ki67 positive hepatocytes and genes present in changed pathways. Asterisks indicate significance levels: ${ }^{*} P<0.05,{ }^{* *} P<0.01$. N.S., not significant. CLV, caudal liver volume (i.e. non-embolized liver volume).

\section{Discussion}

We previously reported that the FXR agonist OCA promotes liver growth following PVE in rabbits. In this study, we demonstrate that OCA improves bile salt homeostasis after PVE, likely through regulating hepatic synthesis and export of bile salts. Opposite relationships between hepatic transcripts involved in bile salt synthesis (Cyp7a1, negatively correlated) and bile salt export (S/c51b, positively correlated) and CLV gain 
were apparent. Our findings support a contribution of restored homeostatic control of bile salt levels, in OCA-augmented liver growth in rabbits undergoing PVE. Furthermore, candidate secreted factors (e.g. Bmp3, Ihh) that may be involved in enhanced liver growth were identified on the basis of transcriptional regulation by OCA and correlation with liver growth parameters.

The PVE procedure redistributes the portal blood flow to the non-embolized liver lobe which is accordingly exposed to the entire pool of bile salts returning from the intestines. Three hours after PVE, serum bile salts levels were elevated 8 to 9 -fold, and this likely reflects systemic spill-over from the portal circulation due to limited spare capacity for bile salt extraction by the non-embolized lobe that comprises only $24 \%$ of total liver volume. Earlier normalization of total and individual bile salt levels after PVE was apparent in OCA-treated animals (Figure 1 and 3). The reason may be that OCA accelerates liver growth, consequently enhancing the capacity of the non-embolized liver lobe to extract portal bile salts. Improved bile salt homeostasis by OCA was also reflected by reduced bile salt content in the non-embolized liver lobe, which may be caused by decreased hepatic synthesis (downregulated expression of Cyp7a1) and enhanced basolateral export (upregulated expression of $S / c 51 a / b$ ) of bile salt. These findings are in line with reported actions of OCA, including observations in patients with cholestatic liver disease ${ }^{31,32}$, and result in a shorter exposure of hepatocytes to potentially harmful bile salts such as LCA.

Serum levels of LCA were lower after PVE in OCA-treated animals, and showed a strong inverse correlation with CLV gain and number of $\mathrm{Ki} 67^{+}$hepatocytes. Likewise, serum levels of HDCA were consistently lower following PVE in the OCA group (Fig. 3). HDCA was previously reported to inhibit proliferation of epithelial cells in the intestine by interfering with the phosphoinositide 3-kinase (PI3K)/AKT pathway that controls the cell cycle, and is also a critical player in early events after liver regeneration triggered by $\mathrm{PH}^{33,34}$. Fractional bile salt composition of individual bile salt species did not show major shifts during the course of PVE, except for HDCA that became a minor fraction after PVE in OCA-treated animals. It is worth exploring whether this is accompanied by enhanced hepatic PI3K signaling in follow-up studies. After PVE, the overall hydrophobicity index of serum bile salts increased in both groups to the same extent. This is indicative for a more cytotoxic bile salt composition after PVE. Taking absolute levels into account, potential detrimental effects of bile salt amount and composition, are expected to be less in OCA-treated animals. The aggregated findings 
indicate that OCA-mediated improvement of bile salt homeostasis contributes to augmented PVE-induced liver growth.

Targeted transcript analysis revealed that OCA induces expression of $C d c 25 b$ in the non-embolized lobe but not in the embolized lobes (Fig. 5B). Cdc25b is a phosphatase that is required for entry into mitosis, and its expression is under control of Fom $1 \mathrm{~b}$, an FXR target gene. In order to identify additional candidate factors that may mediate OCA-augmented liver growth after PVE, RNA isolated from the terminal ileum, the proliferating caudal liver lobe, and the atrophic embolized liver lobes was subjected to RNA sequencing. Among differentially expressed genes (DEGs), candidates were identified based on correlations with liver growth parameters (CLV gain, number of $\mathrm{Ki} 67^{+}$hepatocytes) and an extracellular location of the gene product (e.g. a secreted factor with preferably a signaling activity).

Pathway enrichment analyses indicated that the 'C1q complex' was most significantly affected by OCA in the ileum, with each of the three subunits of this complex upregulated by OCA treatment. Of these, C1qa was positively correlated with hepatocyte proliferation (Fig 6C). The C1q complex was reported to activate mitogenactivated protein kinases (MAPKs) and PI3K/AKT signaling, and resulted in malignant progression of HepG2 hepatoma cells ${ }^{35}$. $\mathrm{C} 1 \mathrm{q}$ is also a constituent of the $\mathrm{C} 1$ complex of the complement system, a system that is intimately linked with liver repair after injury and liver regeneration after $\mathrm{PH}{ }^{21}$. In addition, we observed an inverse association between signaling factor Bmp3 and liver growth. Bmp3 is a member of the bone morphogenetic protein (BMP) family that is linked to the regenerative process of the liver 22. BMP family members are transcriptionally inhibited after $\mathrm{PH}$ in mice ${ }^{36}$. Furthermore, BMP receptor type 1A (BMPR1A) deficient mice display an augmented hepatocyte proliferation after $\mathrm{PH}^{37}$.

In the non-embolized lobe, 'steroid metabolic process' and 'positive regulation of steroid biosynthetic process' were the pathways most significantly affected by OCA treatment. These pathways contain genes (e.g. Cyp7a1 and S/c51b) that were also included in our targeted transcript analysis, and showed a strong correlation with liver growth parameters. In the embolized lobe, OCA induced expression of Ihh that tended to be positively related to hepatocyte proliferation in the non-embolized lobe $(p=0.058)$. Ihh is a cell signaling protein of the hedgehog family that plays a central role mediating liver regeneration in an experimental ALPPS model ${ }^{30}$. Ihh administration combined 
with portal vein ligation induced ALPPS-like acceleration of liver growth, however, blocking Ihh signaling markedly inhibited ALPPS-induced liver regeneration ${ }^{30}$.

This study makes it conceivable that improved bile salt homeostasis contributes to OCA-induced acceleration of liver hypertrophy after PVE. Clinically, OCA may be beneficial to increase FLR volume gain in patients before partial liver resection, thus decreasing the risks of postoperative liver failure and death. In addition, patients scheduled for liver surgery often have damaged liver parenchyma. OCA has already been shown to improve histological features (hepatic steatosis and fibrosis, hepatocellular ballooning) and secretory function in patients with metabolic or cholestatic liver disease ${ }^{32,38}$. A large body of safety data in humans is available for OCA from ongoing clinical trials, including long-term safety IV studies 14, 31, 38. Application of OCA in surgical patients holds promise. However, an essential additional step would be the demonstration of lack of effects of OCA on tumor progression in the embolized segments in a preclinical model.

In conclusion, OCA restores bile salt homeostasis, likely through regulating hepatic bile salt synthesis and export, and this contributes to augmented liver hypertrophy induced by PVE. By accelerating growth of a small FLR, OCA may be promising to shorten the interval between PVE and partial liver resection in patients scheduled for extended liver resection.

\section{Acknowledgements}

We are indebted to Intercept Pharmaceuticals for funding this investigator-initiated study. We are grateful to Loes Nijssen for analysis of serum bile salt composition. 


\section{References}

1. Forbes SJ, Newsome PN. Liver regeneration - mechanisms and models to clinical application. Nat Rev Gastroenterol Hepatol. 2016;13:473-85.

2. Dasari BVM, Hodson J, Roberts KJ, Sutcliffe RP, Marudanayagam R, Mirza DF, et al. Developing and validating a pre-operative risk score to predict post-hepatectomy liver failure. HPB (Oxford). 2019;21:539-46.

3. Olthof PB, Wiggers JK, Groot Koerkamp B, Coelen RJ, Allen PJ, Besselink MG, et al. Postoperative Liver Failure Risk Score: Identifying Patients with Resectable Perihilar Cholangiocarcinoma Who Can Benefit from Portal Vein Embolization. J Am Coll Surg. 2017;225:387-94.

4. Olthof PB, Aldrighetti L, Alikhanov R, Cescon M, Groot Koerkamp B, Jarnagin WR, et al. Portal Vein Embolization is Associated with Reduced Liver Failure and Mortality in High-Risk Resections for Perihilar Cholangiocarcinoma. Ann Surg Oncol. 2020.

5. Hoekstra LT, van Lienden KP, Doets A, Busch OR, Gouma DJ, van Gulik TM. Tumor progression after preoperative portal vein embolization. Ann Surg. 2012;256:812-7; discussion 7-8.

6. Huang W, Ma K, Zhang J, Qatanani M, Cuvillier J, Liu J, et al. Nuclear receptor-dependent bile acid signaling is required for normal liver regeneration. Science. 2006;312:233-6.

7. Uriarte I, Fernandez-Barrena MG, Monte MJ, Latasa MU, Chang HC, Carotti S, et al. Identification of fibroblast growth factor 15 as a novel mediator of liver regeneration and its application in the prevention of post-resection liver failure in mice. Gut. 2013;62:899-910.

8. Otao R, Beppu T, Isiko T, Mima K, Okabe H, Hayashi H, et al. External biliary drainage and liver regeneration after major hepatectomy. Br J Surg. 2012;99:1569-74.

9. Hoekstra LT, van Lienden KP, Schaap FG, Chamuleau RA, Bennink RJ, van Gulik TM. Can plasma bile salt, triglycerides, and apoA-V levels predict liver regeneration? World J Surg. 2012;36:2901-8.

10. Koelfat KVK, van Mierlo KMC, Lodewick TM, Bloemen JG, van der Kroft G, Amygdalos I, et al. Bile Salt and FGF19 Signaling in the Early Phase of Human Liver Regeneration. Hepatology Communications. 2021:12.

11. Olthof PB, Huisman F, Schaap FG, van Lienden KP, Bennink RJ, van Golen RF, et al. Effect of obeticholic acid on liver regeneration following portal vein embolization in an experimental model. Br J Surg. 2017;104:590-9.

12. Loomba R, Sanyal AJ, Kowdley KV, Terrault N, Chalasani NP, Abdelmalek MF, et al. Factors Associated With Histologic Response in Adult Patients With Nonalcoholic Steatohepatitis. Gastroenterology. 2019;156:88-95.e5.

13. Siddiqui MS, Van Natta ML, Connelly MA, Vuppalanchi R, Neuschwander-Tetri BA, Tonascia J, et al. Impact of obeticholic acid on the lipoprotein profile in patients with non-alcoholic steatohepatitis. J Hepatol. 2020;72:25-33.

14. Kowdley KV, Vuppalanchi R, Levy C, Floreani A, Andreone P, LaRusso NF, et al. A randomized, placebo-controlled, phase II study of obeticholic acid for primary sclerosing cholangitis. J Hepatol. 2020.

15. Modica S, Murzilli S, Moschetta A. Characterizing Bile Acid and Lipid Metabolism in the Liver and Gastrointestinal Tract of Mice. Curr Protoc Mouse Biol. 2011;1:289-321.

16. Love MI, Huber W, Anders S. Moderated estimation of fold change and dispersion for RNA-seq data with DESeq2. Genome Biol. 2014;15:550. 
17. Zhou Y, Zhou B, Pache L, Chang M, Khodabakhshi AH, Tanaseichuk O, et al. Metascape provides a biologist-oriented resource for the analysis of systems-level datasets. Nat Commun. 2019;10:1523.

18. Heuman DM. Quantitative estimation of the hydrophilic-hydrophobic balance of mixed bile salt solutions. J Lipid Res. 1989;30:719-30.

19. Cheng J, Fang ZZ, Kim JH, Krausz KW, Tanaka N, Chiang JY, et al. Intestinal CYP3A4 protects against lithocholic acid-induced hepatotoxicity in intestine-specific VDR-deficient mice. J Lipid Res. 2014;55:455-65.

20. Pellicciari R, Fiorucci S, Camaioni E, Clerici C, Costantino G, Maloney PR, et al. 6alpha-ethylchenodeoxycholic acid (6-ECDCA), a potent and selective FXR agonist endowed with anticholestatic activity. J Med Chem. 2002;45:3569-72.

21. Thorgersen EB, Barratt-Due A, Haugaa H, Harboe M, Pischke SE, Nilsson PH, et al. The Role of Complement in Liver Injury, Regeneration, and Transplantation. Hepatology. 2019;70:725-36.

22. Herrera B, Addante A, Sánchez A. BMP Signalling at the Crossroad of Liver Fibrosis and Regeneration. Int J Mol Sci. 2017;19.

23. Lei H, Wang J, Lu P, Si X, Han K, Ruan T, et al. BMP10 inhibited the growth and migration of gastric cancer cells. Tumour Biol. 2016;37:3025-31.

24. Yuan YM, Ma N, Zhang EB, Chen TW, Jiang H, Yin FF, et al. BMP10 suppresses hepatocellular carcinoma progression via PTPRS-STAT3 axis. Oncogene. 2019;38:7281-93.

25. Zhou J, Sun X, Yang L, Wang L, Ran G, Wang J, et al. Hepatocyte nuclear factor 4a negatively regulates connective tissue growth factor during liver regeneration. Faseb j. 2020;34:4970-83.

26. Ujike K, Shinji T, Hirasaki S, Shiraha H, Nakamura M, Tsuji T, et al. Kinetics of expression of connective tissue growth factor gene during liver regeneration after partial hepatectomy and Dgalactosamine-induced liver injury in rats. Biochem Biophys Res Commun. 2000;277:448-54.

27. Martineau C, Najyb O, Signor C, Rassart É, Moreau R. Apolipoprotein D deficiency is associated to high bone turnover, low bone mass and impaired osteoblastic function in aged female mice. Metabolism. 2016;65:1247-58.

28. Bidinotto LT, de Cicco RL, Vanegas JE, Santucci-Pereira J, Vanden Heuvel JP, Washington S, et al. Fish oil alters tamoxifen-modulated expression of mRNAs that encode genes related to differentiation, proliferation, metastasis, and immune response in rat mammary tumors. Nutr Cancer. 2012;64:991-9.

29. Cai Y, Zheng H, Gong W, Che Y, Jiang B. The role of hedgehog signaling pathway in liver regeneration. Hepatogastroenterology. 2011;58:2071-6.

30. Langiewicz M, Schlegel A, Saponara E, Linecker M, Borger P, Graf R, et al. Hedgehog pathway mediates early acceleration of liver regeneration induced by a novel two-staged hepatectomy in mice. J Hepatol. 2017;66:560-70.

31. Hirschfield GM, Mason A, Luketic V, Lindor K, Gordon SC, Mayo M, et al. Efficacy of obeticholic acid in patients with primary biliary cirrhosis and inadequate response to ursodeoxycholic acid. Gastroenterology. 2015;148:751-61.e8.

32. Kjærgaard K, Frisch K, Sørensen M, Munk OL, Hofmann AF, Horsager J, et al. Obeticholic acid improves hepatic bile acid excretion in patients with primary biliary cholangitis. J Hepatol. 2021;74:58-65.

33. Song M, Yang Q, Zhang F, Chen L, Su H, Yang X, et al. Hyodeoxycholic acid (HDCA) suppresses intestinal epithelial cell proliferation through FXR-PI3K/AKT pathway, accompanied by alteration 


\section{Chapter 4}

of bile acids metabolism profiles induced by gut bacteria. Faseb j. 2020;34:7103-17.

34. Jackson LN, Larson SD, Silva SR, Rychahou PG, Chen LA, Qiu S, et al. PI3K/Akt activation is critical for early hepatic regeneration after partial hepatectomy. Am J Physiol Gastrointest Liver Physiol. 2008;294:G1401-10.

35. Lee JH, Poudel B, Ki HH, Nepali S, Lee YM, Shin JS, et al. Complement C1q stimulates the progression of hepatocellular tumor through the activation of discoidin domain receptor 1 . Sci Rep. 2018;8:4908.

36. Otu HH, Naxerova K, Ho K, Can H, Nesbitt N, Libermann TA, et al. Restoration of liver mass after injury requires proliferative and not embryonic transcriptional patterns. J Biol Chem. 2007;282:11197-204.

37. Do N, Zhao R, Ray K, Ho K, Dib M, Ren X, et al. BMP4 is a novel paracrine inhibitor of liver regeneration. Am J Physiol Gastrointest Liver Physiol. 2012;303:G1220-7.

38. Neuschwander-Tetri BA, Loomba R, Sanyal AJ, Lavine JE, Van Natta ML, Abdelmalek MF, et al. Farnesoid $X$ nuclear receptor ligand obeticholic acid for non-cirrhotic, non-alcoholic steatohepatitis (FLINT): a multicentre, randomised, placebo-controlled trial. Lancet. 2015;385:956-65. 
Supplementary Table S1. Primer sequences.

\begin{tabular}{|c|c|c|}
\hline Gene & Forward sequence & Reverse sequence \\
\hline $36 B 4^{t}$ & CCTCGTGAGAGTGACATCGT & CGCCCACGATGAAGCATTTT \\
\hline$H P R T^{\dagger}$ & GACCAGTCAACAGGGGACAT & ATCCAACAAAGTCTGGCCTGT \\
\hline$G A P D H^{+}$ & СCACTACATGGTCTACATGTTCC & TCACCCCACTTGATGTTGGC \\
\hline FXR & ACAAGTGACGTCGACAACGA & AGGTCTGAAACCCTGGCAAC \\
\hline$S H P$ & GCCCCAAGGAATACGCCTAC & CCGGAATGGACTTGAGGGTG \\
\hline NTCP & CCTTGGCCATCTTGGTCTGT & ATCCCCAGGGCTAAGAAGGT \\
\hline ASBT & TGGTACAGGTGCCGAACAG & GACTCCGGCTCTGTTTTGGT \\
\hline FGFR4 & GAAAACCAGCAATGGCCGC & GAGCCCCCAAGTGTGAAGAT \\
\hline$K L B^{*}$ & GAGAACGGCTGGTTCACAGA & TCGAAGCCATCCAGGAGAGA \\
\hline CYP7A1 & ATATGATGAGGAGCTCTGAAGC & GGGACTCCTTGATGATGCTGT \\
\hline CYP8B1 & CACCTGATGGGACATGGCTT & CAGGTAGCCAGCCTTGAACA \\
\hline SLC51A & CCCCACGGTGGTGTCTATAA & GCCTTCCACCATGACCATCA \\
\hline SLC51B & TGGGAACAGGAGCCAGAAAC & CGTCAGGGCAAGGATGGAAT \\
\hline SLCO1B3 & AGATGCTGGGGAAAGGTTGT & TGCAAAGCCAATGATAGGGC \\
\hline$\angle F A B P$ & ATGGGGAAGGTCAAGACAGT & ATCCGCTTGCTGATTCGCTT \\
\hline FOXM1B & CCGTCACAACCTGTCTCTCC & GCTGCTGTGATTCCAAGTGC \\
\hline$C D C 25 B$ & GACTTTTGCCCGTCTCAGGA & GGAGCCCCCTAGGACACTAA \\
\hline MKI67 & CGCCTGCTCGTTCGGAAG & TATACAACACTGCCTCCTGCTC \\
\hline PCNA & АССТCACCAGCATGTCCAAAAT & GGTCTCGGCATATACGTGCAA \\
\hline
\end{tabular}

${ }^{\dagger}$ Reference genes. 'Synonyms: KLB, $\beta$-Klotho. 
Chapter 4

Supplementary Table S2. Correlations between bile salt-related parameters and liver growth-related parameters at day 3 .

\begin{tabular}{|c|c|c|c|c|}
\hline \multirow{2}{*}{ Variables } & \multicolumn{2}{|c|}{ CLV increase (\%) } & \multicolumn{2}{|c|}{ Ki67+ hepatocytes (\%)* } \\
\hline & $\rho$ & $P$ value & $\rho$ & $P$ value \\
\hline Non-embolized liver lobe TBS (nmol/g) & -0.357 & 0.255 & -0.123 & 0.704 \\
\hline Embolized liver lobes TBS (nmol/g) & -0.364 & 0.245 & -0.091 & 0.778 \\
\hline Serum TBS $(\mu \mathrm{mol} / \mathrm{L})$ & -0.518 & 0.014 & +0.056 & 0.863 \\
\hline C4 (ng/mL) & -0.007 & 0.983 & -0.200 & 0.534 \\
\hline $\mathrm{DCA}(\mathrm{nmol} / \mathrm{L})$ & -0.527 & 0.012 & -0.340 & 0.280 \\
\hline GDCA (nmol/L) & -0.373 & 0.087 & -0.172 & 0.594 \\
\hline $\mathrm{HDCA}(\mathrm{nmol} / \mathrm{L})$ & -0.491 & 0.020 & -0.532 & 0.075 \\
\hline $\mathrm{CDCA}(\mathrm{nmol} / \mathrm{L})$ & -0.377 & 0.084 & -0.077 & 0.812 \\
\hline $\mathrm{CA}(\mathrm{nmol} / \mathrm{L})$ & -0.315 & 0.154 & -0.119 & 0.712 \\
\hline $\mathrm{GCA}(\mathrm{nmol} / \mathrm{L})$ & -0.266 & 0.232 & -0.172 & 0.594 \\
\hline LCA (nmol/L) & -0.726 & $<0.001$ & -0.718 & 0.009 \\
\hline GLCA (nmol/L) & -0.139 & 0.536 & -0.189 & 0.556 \\
\hline UDCA (nmol/L) & -0.220 & 0.326 & -0.014 & 0.966 \\
\hline $\mathrm{OCA}(\mathrm{nmol} / \mathrm{L})$ & -0.536 & 0.089 & -0.696 & 0.125 \\
\hline GOCA (nmol/L) & -0.109 & 0.750 & -0.812 & 0.050 \\
\hline TOCA (nmol/L) & +0.214 & 0.610 & +0.500 & 0.667 \\
\hline Hydrophobicity index & +0.220 & 0.326 & +0.235 & 0.463 \\
\hline
\end{tabular}

${ }^{*}$ Correlations are based on values derived from 12 animals, except for OCA $(n=6)$. CLV, caudal liver volume (non-embolized liver volume); TBS, total bile salt; C4, 7- $\alpha$-hydroxy-4-cholesten-3-one. 


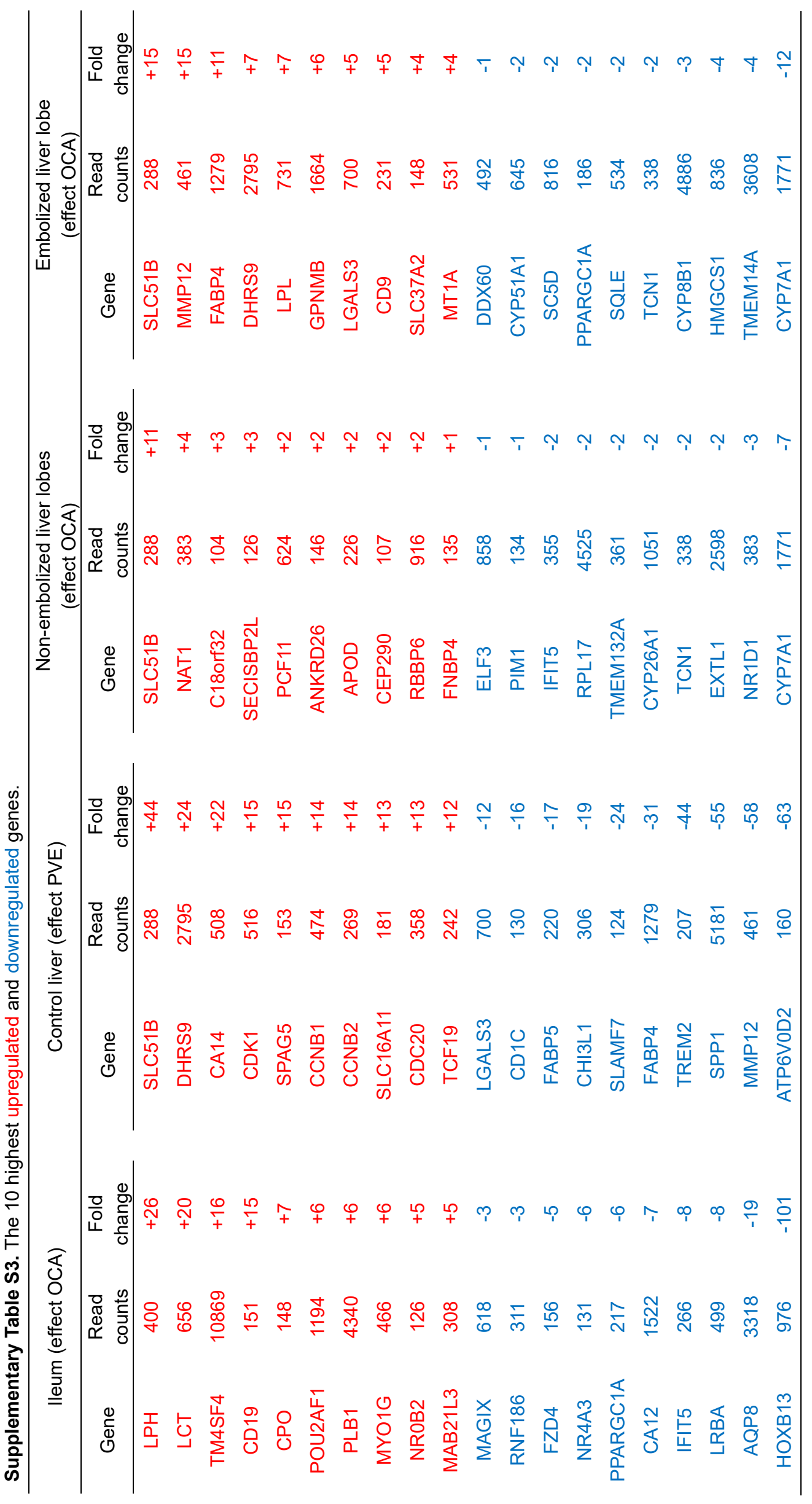




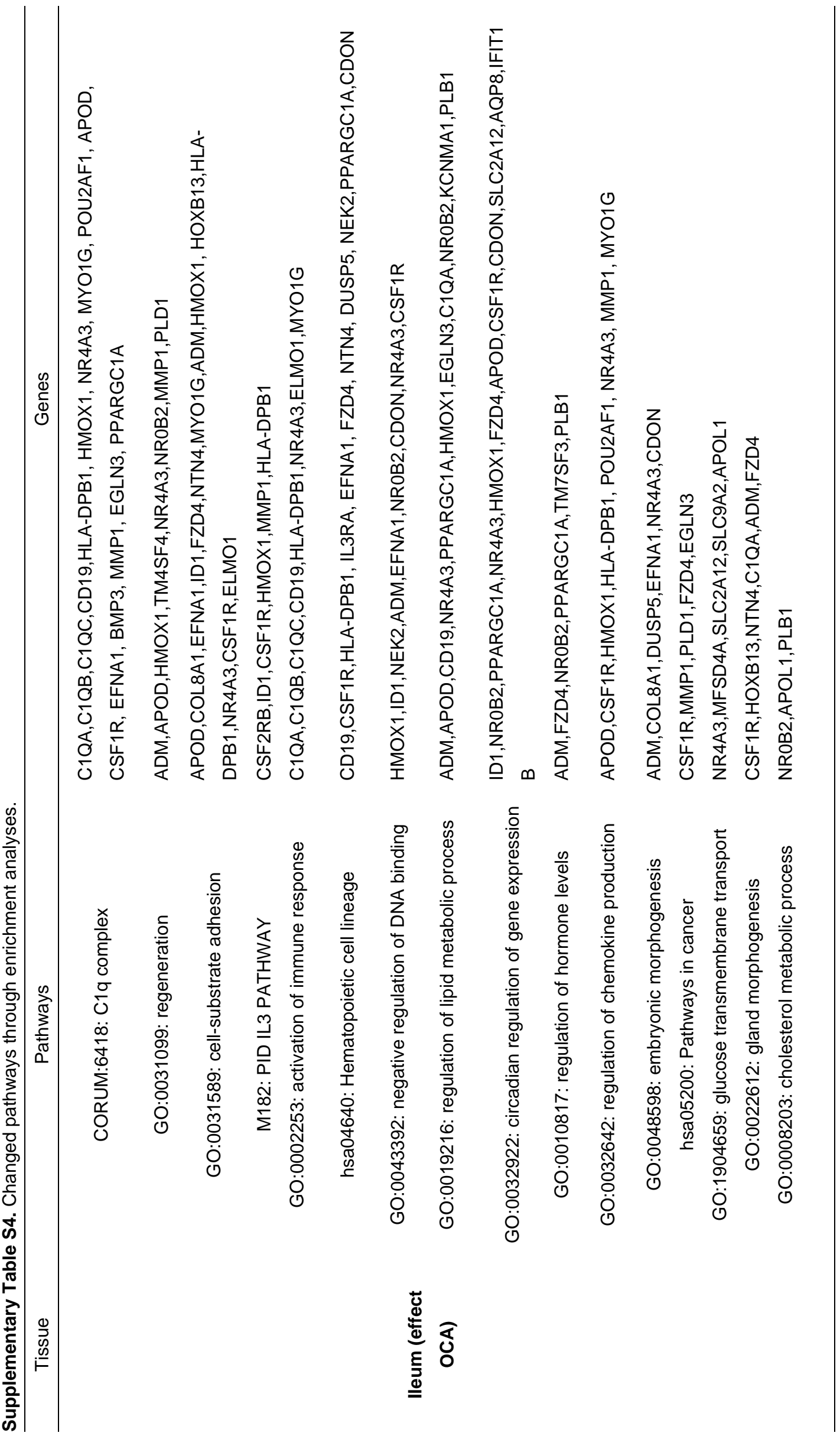




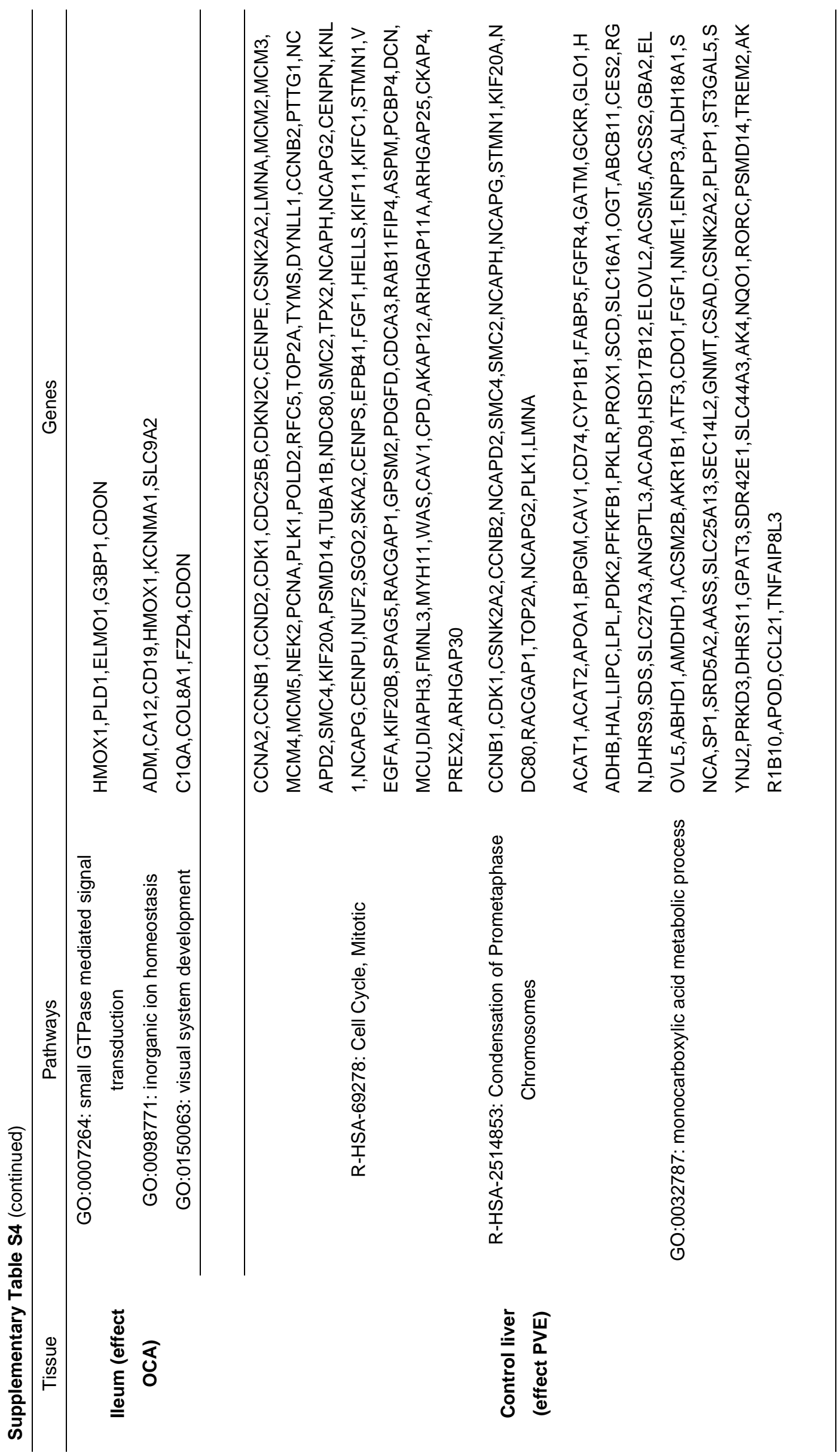




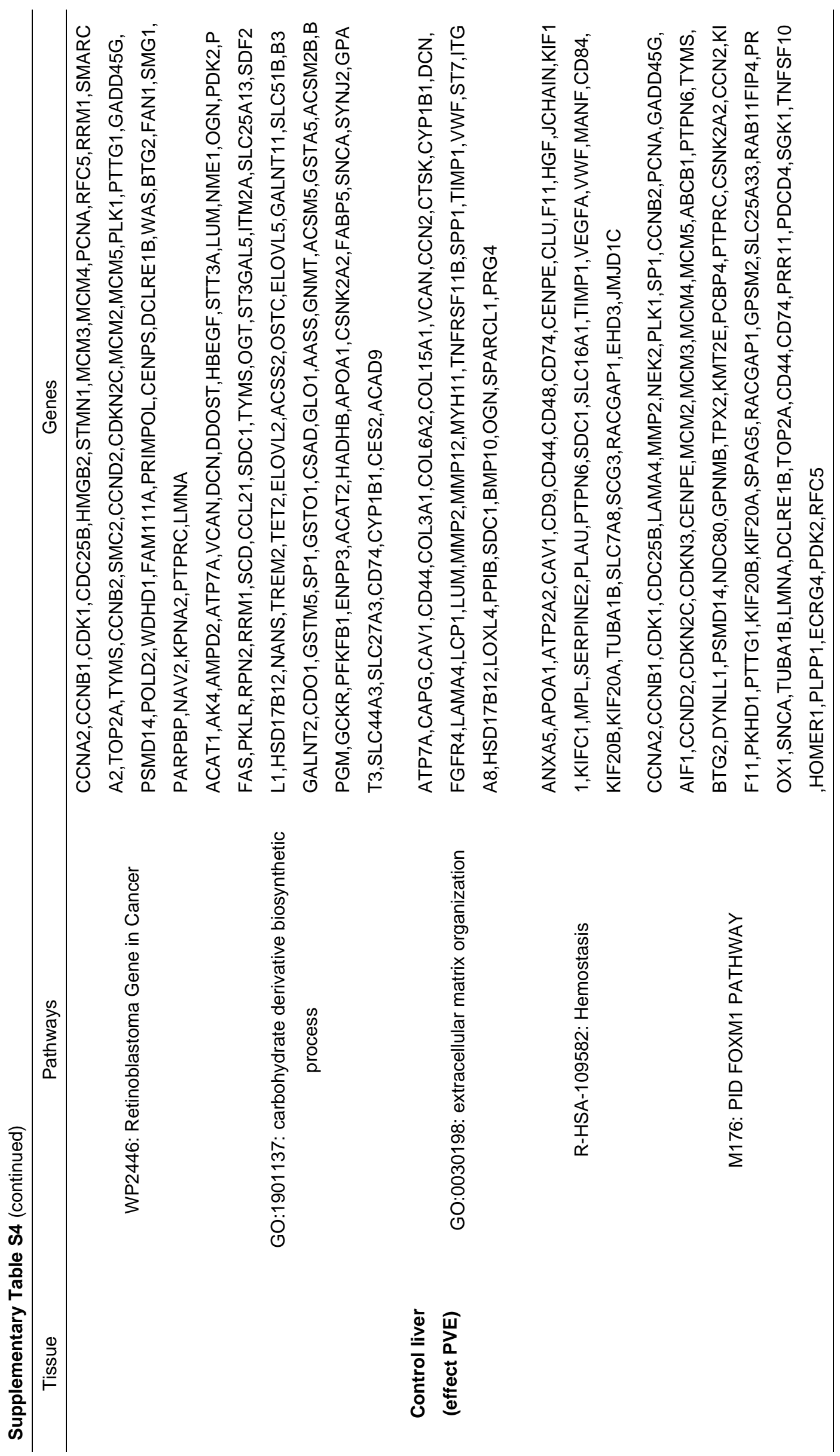




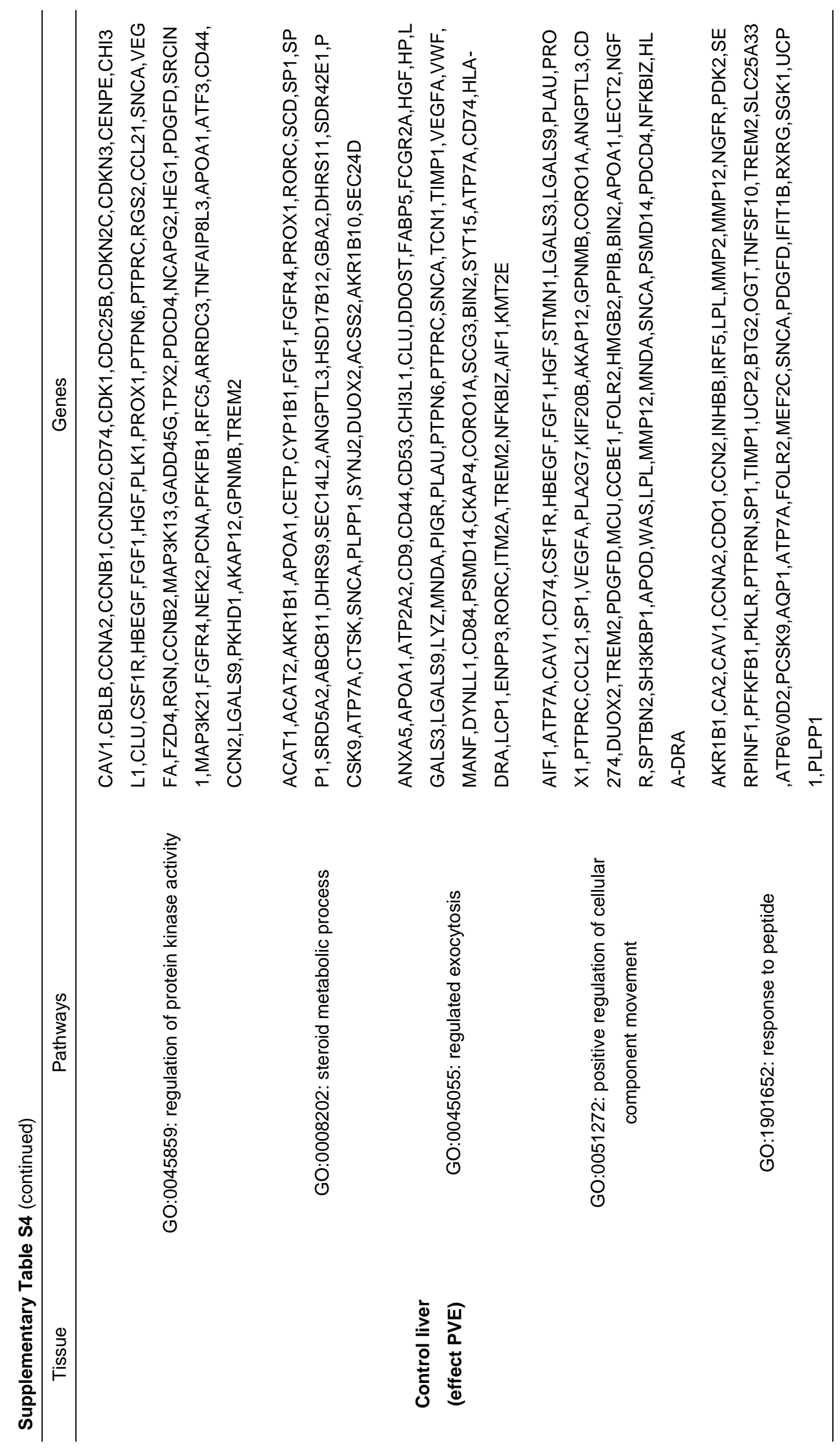




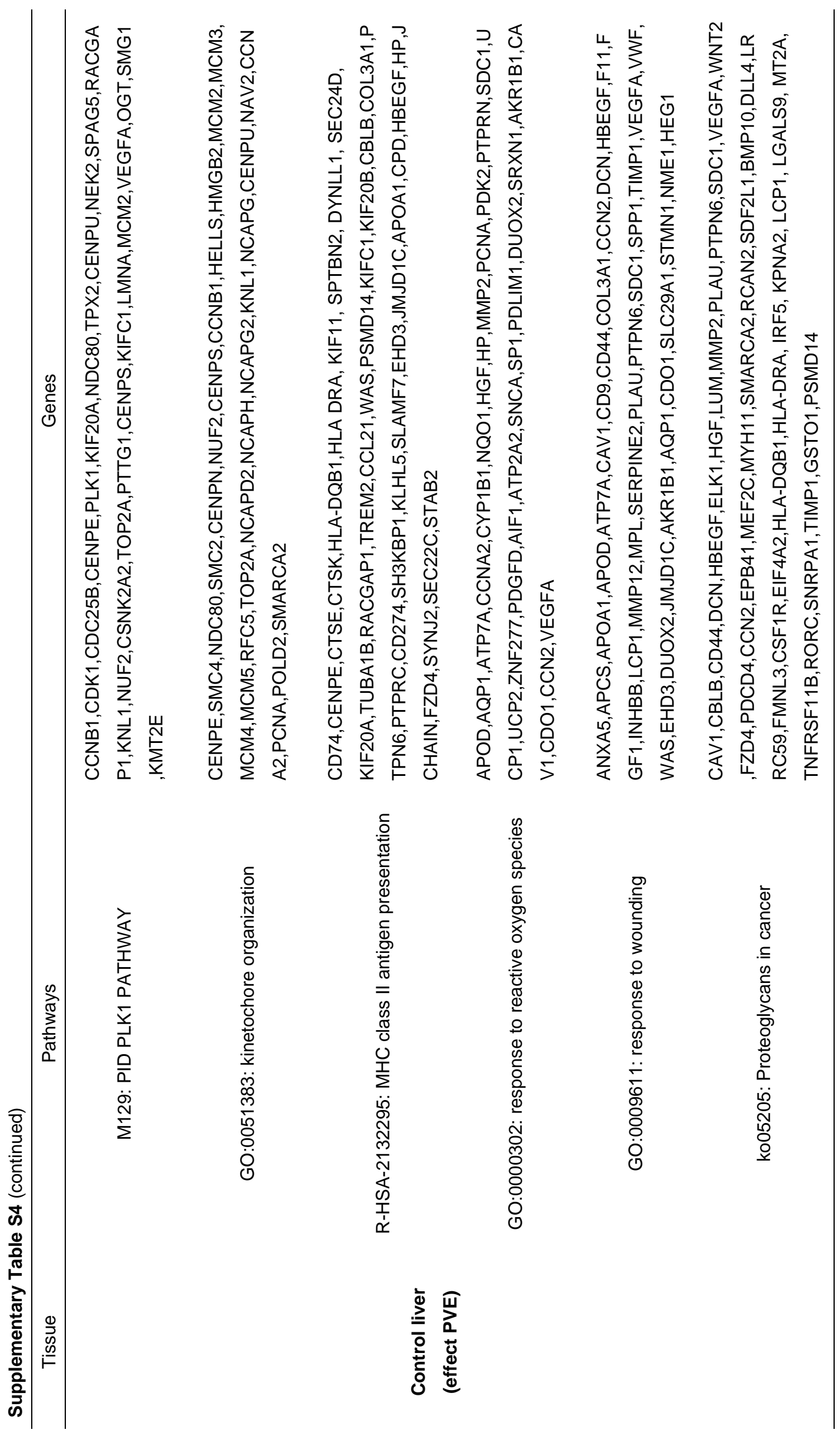




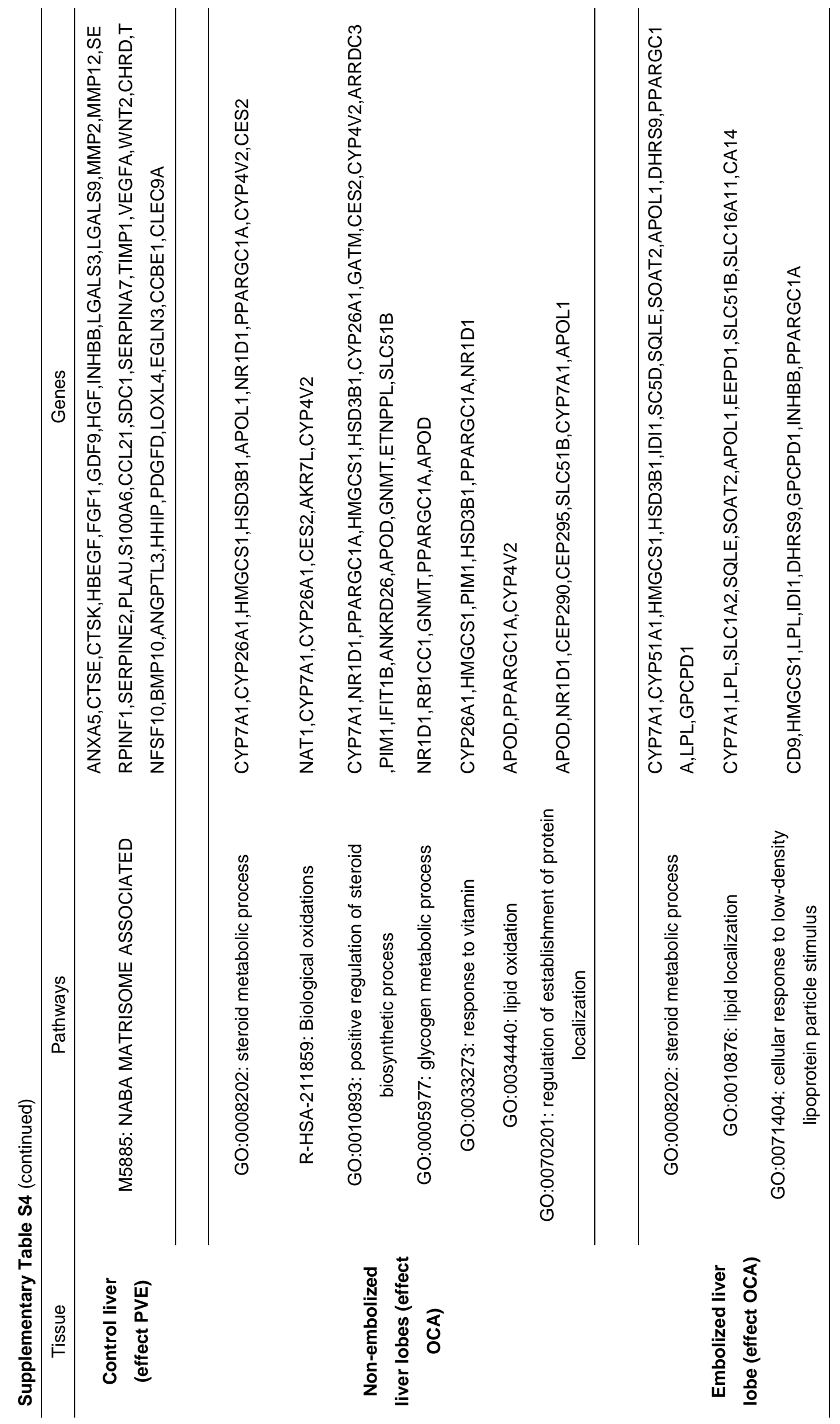




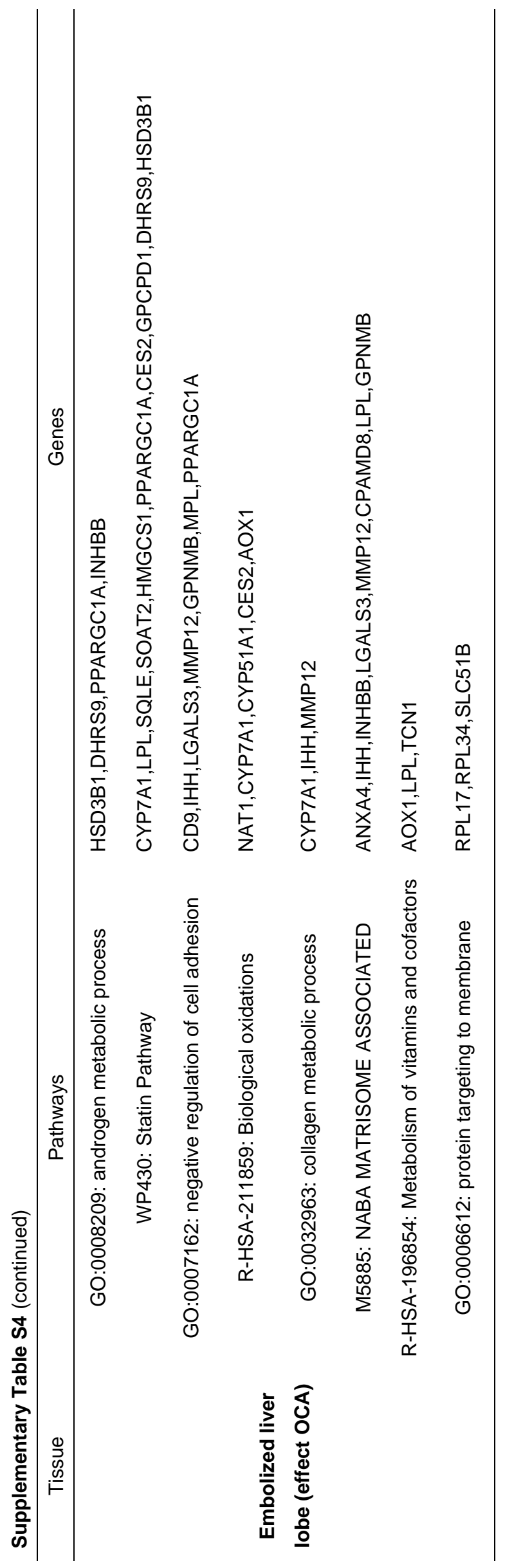




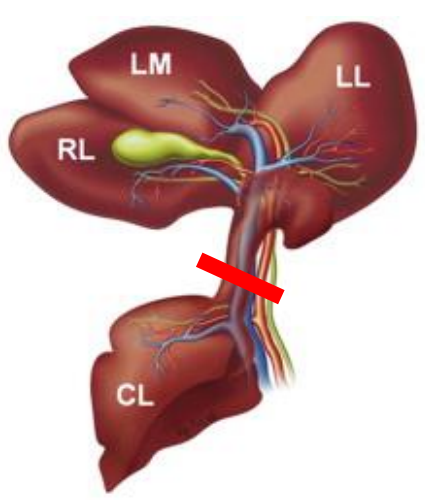

Supplementary Figure $\mathbf{S 1}$. Portal vein embolization of liver in rabbit. Caudal liver lobe $(C L)$ was non-embolized and cranial liver lobes (RL, LM, and LL) were embolized.
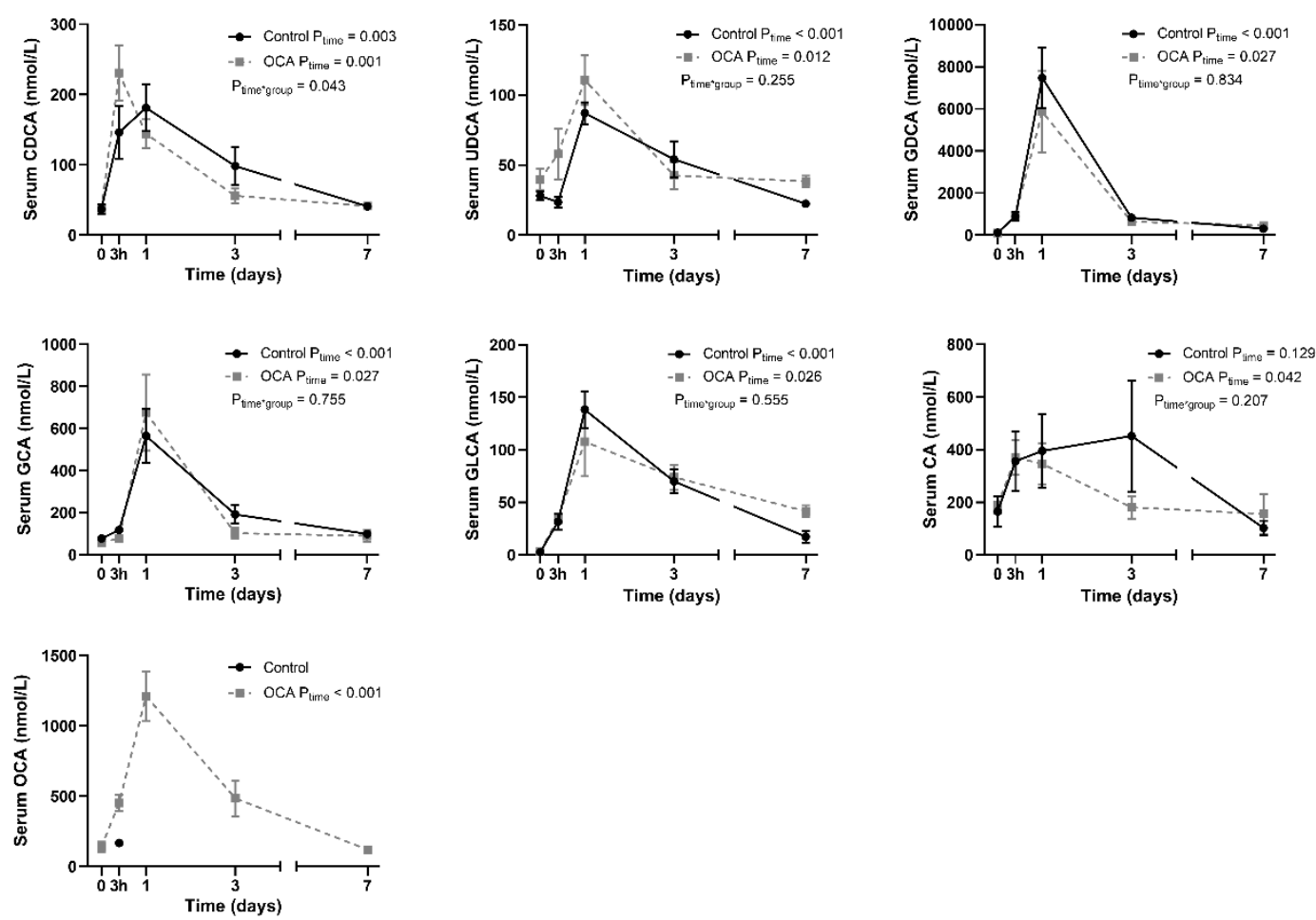

Supplementary Figure S2. Change of serum individual bile acid species during the course of experiment. Longitudinal changes were evaluated by mixed effect model $(n=11$ per group). Differences between OCA-treated animals and control animals were analyzed with the Mann-Whitney $U$ test. Data is depicted as mean \pm SEM. $P$ values are depicted. Asterisks indicate significance levels: ${ }^{* *} \mathrm{P}<0.01$, ${ }^{* *} \mathrm{P}<0.001$. HDCA, deoxycholic acid; $\mathrm{CA}$, cholic acid; UDCA, ursodeoxycholic acid; GDCA, glycodeoxycholic acid; GCA, glycocholic acid; GLCA, glycolithocholic acid; OCA, obeticholic acid. 
Chapter 4

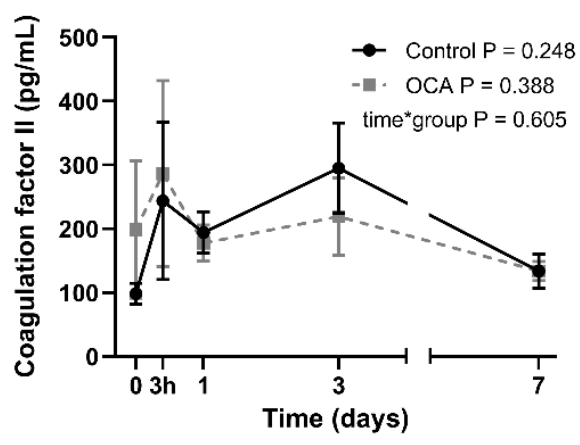

Supplementary Figure S3. OCA does not affect liver synthetic function. Plasma coagulation factor II levels were not different between OCA-treated animals and control animals during the experiment ( $n=11$ per group). Longitudinal changes were evaluated by mixed effect model. Data is depicted as mean \pm SEM. $P$ values are depicted.
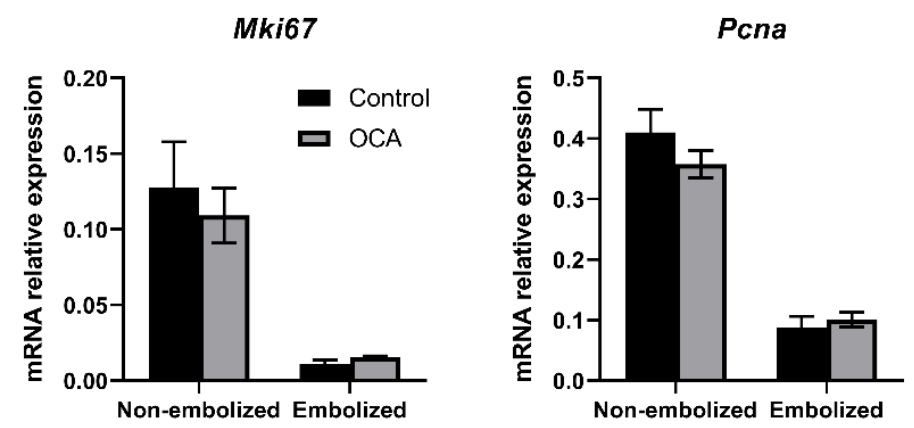

Supplementary Figure S4. OCA does not induce mRNA expression of Mki67 and Pcna. Differences between OCA-treated animals and control animals were analyzed with the MannWhitney $U$ test ( $n=6$ per group). 


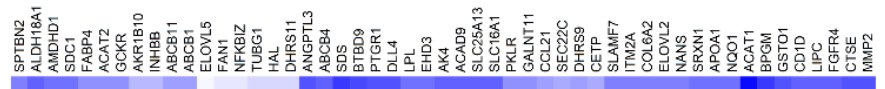

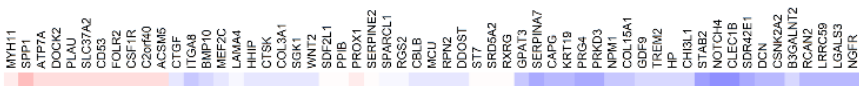

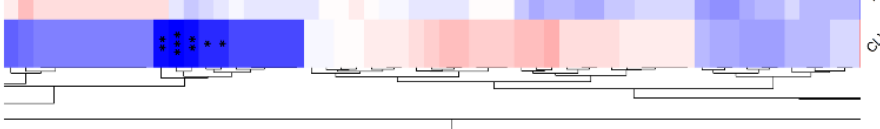

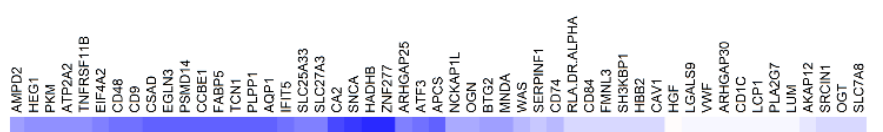

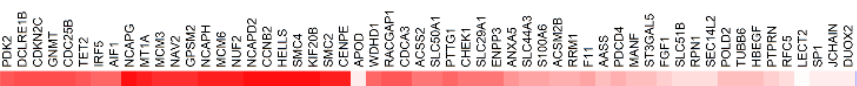

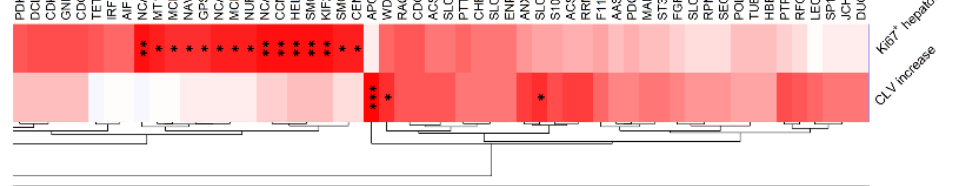

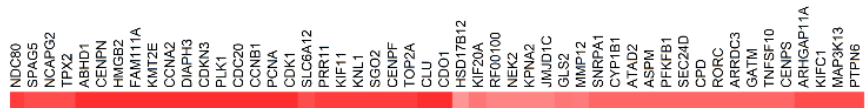

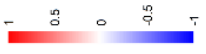

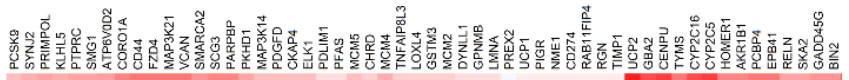





\section{Chapter 5}

\section{No influence of cholestasis on portal vein embolization-induced hypertrophy of the future liver remnant}

Xinwei Chang ${ }^{*}$, Remon Korenblik* Bram Olij, Bob Knapen, Christiaan van der Leij, Lloyd Brandts, Daniel Heise, Marcel den Dulk, Ulf Neumann, Frank G. Schaap, Ronald van Dam, Steven W.M. Olde Damink ${ }^{*}$ First authors contributed equally. 


\section{Abstract}

Background: In the pre-clinical setting, hepatocellular bile salt accumulation impairs liver regeneration following partial hepatectomy. Here, we study the impact of cholestasis on portal vein embolization (PVE)-induced hypertrophy of the future liver remnant (FLR).

Methods: Patients were enrolled with perihilar cholangiocarcinoma ( $\mathrm{pCCA}$ ) or colorectal liver metastases (CRLM) undergoing PVE before a (extended) right hemihepatectomy. Volume of segments II/III was considered as FLR and assessed on pre- and post-embolization CT scans. The degree of hypertrophy (DH, percentual increase), and kinetic growth rate (KGR, percentage/week) were used to assess PVEinduced hypertrophy.

Results: A total of 50 patients ( 31 CRLM, 19 pCCA) were included. The DH and KGR were similar in patients with PCCA and CRLM, and in PCCA patients with or without hyperbilirubinemia. For patients with PCCA, unilateral drainage in FLR induced a higher $\mathrm{DH}$ than bilateral drainage (6.7 [4.9-7.9] versus 2.7 [1.5-4.2] \%, $\mathrm{p}=0.012$ ). Creactive protein before PVE was negatively correlated with $\mathrm{DH}$ and KGR in patients with pCCA.

Conclusion: There was no influence of cholestasis on FLR hypertrophy in patients undergoing PVE. Unilateral drainage of the FLR resulted in higher liver hypertrophy than bilateral drainage. Elevated inflammation response was associated with impaired liver growth in patients with PCCA. 


\section{Introduction}

Most major hepatectomies are performed to achieve a complete margin-free resection with a curative intention, in patients with hepatobiliary tumors. However, in patients undergoing resection, a small future liver remnant (FLR) volume is associated with a higher incidence of post-hepatectomy liver failure and death ${ }^{1,2}$. In order to achieve a safe liver resection, the FLR volume must be $>30 \%$ in uncompromised livers, $>40 \%$ in chemotherapy-damaged or steatotic livers, and $>50 \%$ in cirrhotic livers ${ }^{3,4}$. In practice, liver resections are commonly performed in patients with underlying liver disease, and an insufficient FLR volume may preclude surgery ${ }^{5}$.

The current standard procedure to induce hypertrophy of the non-embolized liver segments is portal vein embolization (PVE), where the aim is to increase FLR volume prior to resection ${ }^{6}$. PVE has been associated with an increase in FLR volume of 20 $50 \%$ within 3-7 weeks ${ }^{7,8}$. Studies have shown that PVE-induced hypertrophy of the FLR has a beneficial effect on reducing the rates of liver failure and death after major liver resection 2, 9. PVE induces insufficient hypertrophy of the FLR (i.e. failure to proceed to hepatectomy) in approximately $30 \%$ patients with colorectal liver metastasis $(C R L M){ }^{10}$, which might be linked to the formation of collateral blood vessels in embolized segments. For this reason, new techniques are being investigated to induce hypertrophy of the FLR. A novel procedure, i.e. combined Portal and Hepatic Vein Embolization (PVE/HVE), aims at preventing collateral formation and is currently being evaluated by the international DRAGON consortium ${ }^{11}$.

Besides more effective hypertrophy-inducing techniques, investigations are also being conducted to identifying those factors that negatively or positively affect cell growth in the non-embolized liver lobe ${ }^{12,13}$. In that respect, cholestasis is frequently present in patients with hepatobiliary tumors, especially in patients with perihilar cholangiocarcinoma ( $\mathrm{pCCA})$. Previous studies have demonstrated that during cholestasis excessive hepatic bile salt levels occur, which impair the proliferative capacity of hepatocytes ${ }^{14,15}$. By contrast, stimulation of bile salt signaling by agonistic activation of the bile salt receptor Farnesoid X Receptor (FXR) was found to promote liver growth in a rabbit PVE model ${ }^{13}$. A wealth of animal studies have stressed the importance of maintained bile salt homeostasis in the proper progression of liver growth following partial hepatectomy ${ }^{16-18}$. 
A few studies have examined the impact of cholestasis on FLR hypertrophy in patients undergoing PVE, but their findings have been inconclusive ${ }^{8,19}$. One recent study, for example, reported similar hypertrophy of the FLR in patients with a primary biliary malignancy and normal or elevated (i.e. $\geq 50 \mu \mathrm{mol} / \mathrm{L}$ ) serum bilirubin ${ }^{19}$. The aim of the present study was to test the hypothesis that cholestasis impairs FLR hypertrophy in patients undergoing PVE. To this end, PVE-induced FLR hypertrophy was analyzed based on serum bilirubin levels. In addition, we investigated the associations between routine serum biochemical tests and/or biliary drainage, and FLR hypertrophy.

\section{Methods}

\section{Study design}

This study is a bi-center retrospective cohort study. Adult patients ( $\geq 18$ years) with pCCA or CRLM were considered who had undergone PVE prior to (extended) right hepatectomy between January 01, 2016 and December 31, 2019 in the Maastricht University Medical Center (the Netherlands) or in the Uniklinik Aachen (Germany). Patients who had missing liver computed tomography (CT) scans, partial resection of the FLR between PVE and liver volume assessment, or only inadvertent drainage of the tumor bearing segments, were excluded. This study was approved by the institutional review boards of both centers (METC2019-1375 and EK 434/19).

\section{Assessment of liver volume}

Since all included patients were planned for (extended) right hepatectomy, liver segments II and III were consistently part of the FLR, and the volume of these segments was taken as the measure of FLR. Total liver volumes (TLV) and FLR volumes were determined from routine CT scans both prior to and after PVE. If multiple PVE procedures had been attempted to induce sufficient FLR volume gain, the liver volumetry after the last PVE was used. The diameters of the left and right hepatic bile duct were measured on pre-embolization scans, with bile duct dilation indicative for obstructive cholestasis. Additionally, the degree of bile duct dilation was assessed visually as: no, mild, moderate, or severe. CT scans were locally de-identified and volumetry was performed using Syngo.via Liver Analysis, Siemens Healthineers. All volumetries were performed by an investigator $(X C)$, checked by another investigator $(\mathrm{RK})$, and both were proctored by a local interventional radiologist $(\mathrm{CL})$. 


\section{Definitions and data collection}

FLR increase was defined as the difference in FLR volume before (FLR1) and after PVE (FLR2). FLR share (FLR\%) was calculated as the FLR volume divided by TLV. \%Hypertrophy was defined as the difference in FLR volume before and after PVE (FLR2-FLR1) divided by the FLR volume before PVE (FLR1). The degree of hypertrophy $(\mathrm{DH})$ was defined as the difference in FLR share before (FLR1\%) and after PVE (FLR2\%) (DH = FLR2\% - FLR1\%). Kinetic growth rate (KGR) was calculated as $\mathrm{DH}$ divided by the time elapsed in weeks between PVE and the CT volumetry after PVE.

The following main clinical and laboratory variables were retrieved from the patient files: tumor type, histological status of liver parenchyma, occurrence of drainage prior to PVE, and presence of cholangitis prior to PVE. Routine serum biochemistry parameters (bilirubin, albumin, international normalized ratio [INR], gamma-glutamyl transferase [GGT], alkaline phosphatase [ALP], alanine aminotransferase [ALT], aspartate aminotransferase [AST], C-reactive protein [CRP] and white blood cell count) were gathered prior to PVE and -if applicable- prior to biliary drainage. Bilirubin level $\geq 50.0 \mu \mathrm{mol} / \mathrm{L}$ was used as a clinical marker of cholestasis. Confirmation bias was prevented by having a separate researcher $(\mathrm{BO})$ collect the data from the Electronic Health Records. All data were gathered in CASTOR EDC, Amsterdam, a Good Clinical Practice-accepted online data capturing and monitoring system.

\section{Statistical analyses}

All statistical analyses were performed using SPSS 24.0 (IBM, Armonk, New York, USA). Data are presented as median [interquartile range] or frequency (percentage) when appropriate. For continuous data, differences between two groups were compared using the Mann-Whitney $U$ test. Serum biochemistry parameters, assessed sequentially in patients with pCCA before drainage and before PVE, were compared using a Wilcoxon signed rank test. Categorical data was compared using chi-square test or Fisher's exact test as appropriate. Correlations were evaluated by Spearman rank correlation coefficients $(\rho)$. Statistical significance was considered at $p<0.05$.

\section{Results}

\section{Patient characteristics}


A total of 50 patients (CRLM, $n=31 ;$ pCCA, $n=19$ ) were enrolled and their characteristics are shown in Table 1. Patients diagnosed with CRLM had a higher median Charlson comorbidity index than those with $\mathrm{pCCA}$, but were otherwise similar. All patients underwent embolization of the right portal branch, with the intention to undergo (extended) right hemihepatectomy. Four patients received an extended segment IV embolization (Table 1). The technical success of the PVE procedure (successful obstruction of the right portal branch) was achieved in all patients. Two patients with CRLM had more than one PVE procedure. Twenty-nine out of thirty-one patients with CRLM received chemotherapy before PVE, whereas none of the patients with pCCA received chemotherapy.

None of the patients with CRLM were drained, whereas seventeen out of nineteen patients with PCCA underwent biliary drainage prior to PVE. Of those seventeen patients, eleven received unilateral drainage of the FLR, with the remaining six receiving simultaneous drainage of the right hepatic lobe.

\section{pCCA patients remain cholestatic at the time of PVE}

To assess the efficacy of biliary drainage in patients with pCCA, serum biochemistry parameters prior to and after biliary drainage were compared (Supplementary Table 1). Thirteen out of seventeen patients had hyperbilirubinemia before drainage, with bilirubin data missing for one patient. Serum bilirubin levels were reduced 2.6-fold after drainage, but remained above the normal range in 10 patients. After drainage, ALT levels were decreased, and there was a trend towards a reduction of AST levels ( $p=0.026$ and $p=0.087$, respectively). Prior to the PVE procedure, patients with $p C C A$ had higher serum levels of bilirubin, GGT, and ALP than patients with CRLM (Supplementary Table 1), reflecting cholestatic liver injury and its incomplete resolution by drainage.

\section{Liver growth after PVE}

The median time from the initial PVE procedure to volumetric assessment post-PVE was 22 [19-29] days and 20 [16-22] days in patients with CRLM and PCCA, respectively ( $p=0.141$, Table 2 ). The absolute FLR volume increased from 263 [208327] to 363 [322-439] $\mathrm{mL}$ after PVE in patients with CRLM, and from 326 [220-437] to 394 [363-542] $\mathrm{mL}$ in patients with pCCA (Supplementary Fig. 1A). Accordingly, the FLR\% increased significantly after PVE in both groups (Supplementary Fig. 1B). 
Table 1. Baseline characteristics of participants.

\begin{tabular}{|c|c|c|c|}
\hline Characteristics & $\operatorname{CRLM}(n=31)$ & $\operatorname{pCCA}(n=19)$ & $p$ value \\
\hline Age (years) & $65[56-73]$ & 70 [67-76] & 0.070 \\
\hline Gender & & & 0.276 \\
\hline Female & $7(23 \%)$ & $7(37 \%)$ & \\
\hline Male & $24(77 \%)$ & $12(63 \%)$ & \\
\hline BMI $\left(\mathrm{kg} / \mathrm{m}^{2}\right)$ & $25.2[22.8-28.4]$ & 23.4 [22.3-26.3] & 0.448 \\
\hline Diabetes & $6(19 \%)$ & $4(21 \%)$ & 1.000 \\
\hline Charlson comorbidity index & $8[7-9]$ & $6[5-8]$ & $<0.001$ \\
\hline Nontumoral liver parenchyma* & & & 0.946 \\
\hline Normal & $18(62 \%)$ & $11(65 \%)$ & \\
\hline Fibrosis & $5(17 \%)$ & $2(12 \%)$ & \\
\hline Cirrhosis & $1(4 \%)$ & $1(6 \%)$ & \\
\hline Steatosis & $5(17 \%)$ & $3(17 \%)$ & \\
\hline Cholangitis & $0(0 \%)$ & $3(16 \%)$ & \\
\hline \multicolumn{4}{|l|}{ Bismuth-Corlette classification } \\
\hline I & - & $1(5 \%)$ & \\
\hline II & - & $2(11 \%)$ & \\
\hline IIIA & - & $5(26 \%)$ & \\
\hline IIIB & - & $1(5 \%)$ & \\
\hline IV & - & $10(53 \%)$ & \\
\hline Chemotherapy & & & $<0.001$ \\
\hline No & $2(6 \%)$ & $31(100 \%)$ & \\
\hline FOLFOX/XELOX & $23(74 \%)$ & & \\
\hline FOLFIRI/FOLFOXIRI & $5(16 \%)$ & & \\
\hline Other & $1(4 \%)$ & & \\
\hline Type of biliary drainage & & & $<0.001$ \\
\hline No & $31(100 \%)$ & $2(11 \%)$ & \\
\hline PTBD & - & $2(11 \%)$ & \\
\hline EBD & - & $9(47 \%)$ & \\
\hline Both & - & $6(31 \%)$ & \\
\hline \multicolumn{4}{|l|}{ Side of biliary drainage } \\
\hline Unilateral & - & $11(65 \%)$ & \\
\hline Bilateral & - & $6(35 \%)$ & \\
\hline \multicolumn{4}{|l|}{ Hepatic bile duct diameter (mm) } \\
\hline Left & $<3.0$ & $6.3[5.3-10.0]$ & $<0.001$ \\
\hline Right & $<3.0$ & $7.3[5.4-10.7]$ & $<0.001$ \\
\hline Hepatic bile duct dilation (left or right) & & & $<0.001$ \\
\hline No & $31(100 \%)$ & $0(0 \%)$ & \\
\hline Mild+Moderate & - & $8(42 \%)$ & \\
\hline Severe & - & $11(58 \%)$ & \\
\hline Type of PVE & & & 0.629 \\
\hline Right PVE & $29(94 \%)$ & $17(90 \%)$ & \\
\hline Right PVE extended to segment IV & $2(6 \%)$ & $2(10 \%)$ & \\
\hline
\end{tabular}

Data are presented as median [interquartile range] or frequency (percentage). *Histological information is not available for two patients in each group. CRLM, colorectal liver metastasis; pCCA, perihilar cholangiocarcinoma; BMI, body mass index; PTBD, percutaneous transhepatic biliary drainage; EBD, endoscopic biliary drainage; FLR, future liver remnant; PVE, portal vein embolization. 
The absolute FLR volume increase and \%hypertrophy were not different in CRLM and pCCA groups ( $p=0.522$ and $p=0.897$, respectively) (Table 2 ). In addition, patients with CRLM and pCCA had similar DH (5.2 [3.3-6.9] versus 5.7 [3.2-7.4] \%, respectively; $p$ $=0.960)$ and KGR (1.4 [0.9-2.5] versus $1.9[1.0-2.4] \% /$ week, respectively; $p=0.742)$ after PVE (Table 2).

As patients with CRLM had a higher Charlson comorbidity index, a sensitivity analysis was performed, which was done by 1:1 matching based on age, gender, BMI, Charlson comorbidity index, cirrhosis, and presence of diabetes. The characteristics of the matched patients ( $n=10$ in each group) are presented in Supplementary Table 2. Both $\mathrm{DH}$ and KGR were still comparable between patients with CRLM and pCCA (DH: 5.9 [3.8-7.8] versus $5.8[1.8-7.1] \%$, respectively; $p=0.597 ; \mathrm{KGR}: 1.6$ [0.9-2.8] versus 1.6 [0.5-2.4] \%/week, respectively; $p=0.597$ ).

Table 2. Assessment of FLR hypertrophy after PVE between patients with CRLM and pCCA.

\begin{tabular}{lccc}
\hline Variable & CRLM $(n=31)$ & pCCA $(n=19)$ & p value \\
\hline Number of days after PVE & $22[19-29]$ & $20[16-22]$ & 0.141 \\
FLR increase $(\mathrm{mL})$ & $88[53-139]$ & $120[49-165]$ & 0.522 \\
FLR increase per day $(\mathrm{mL} /$ day $)$ & $3.5[1.9-6.9]$ & $4.5[2.4-8.8]$ & 0.299 \\
\%Hypertrophy & $30[19-59]$ & $38[14-65]$ & 0.897 \\
$\%$ Hypertrophy per week & $9.2[6.0-18.3]$ & $10.6[5.2-19.8]$ & 0.667 \\
DH $(\%)$ & $5.2[3.3-6.9]$ & $5.7[3.2-7.4]$ & 0.960 \\
KGR $(\%$ per week) & $1.4[0.9-2.5]$ & $1.9[1.0-2.4]$ & 0.742 \\
\hline
\end{tabular}

Data are presented as median [interquartile range]. CRLM, colorectal liver metastasis; pCCA, perihilar cholangiocarcinoma; PVE, portal vein embolization; FLR, future liver remnant; $\mathrm{DH}$, Degree of hypertrophy; KGR, kinetic growth rate.

\section{Cholestasis does not affect PVE-induced liver growth in pCCA}

After biliary drainage, ten patients with pCCA still had hyperbilirubinemia. These patients had comparable $\mathrm{DH}$ and KGR to patients with bilirubin levels lower than 50 $\mu \mathrm{mol} / \mathrm{L}$ (DH: 5.6 [3.0-7.5] versus 5.7 [2.4-7.0] \%, respectively, $\mathrm{p}=0.806$; KGR: 1.7 [1.02.4] versus 1.9 [0.8-2.4] \%/week, respectively, $p=1.000$ ) (Table 3). Spearman correlation analyses also showed that serum bilirubin levels before drainage ( $\mathrm{pCCA}$ group: $\rho=-0.129, p=0.633$ ) or before PVE (entire cohort: $\rho=-0.023, p=0.879$ ) were not correlated with the DH (Fig. 1A-B, Supplementary Table 4). Likewise, serum bilirubin levels before drainage ( $p C C A$ group: $\rho=+0.097, p=0.72$ ) or before PVE (entire cohort: $\rho=+0.148, p=0.321$ ) were unrelated to KGR. 
In the pCCA group, there were no differences in DH and KGR between patients with mild-moderate bile duct dilation $(n=8)$ and those with severe bile duct dilation $(n=11)$ (DH: 6.0 [4.5-8.5] versus 4.5 [2.4-7.0] \%, respectively, p=0.283; KGR: 2.0 [1.1-2.6] versus $1.6[1.0-2.3] \% /$ week, respectively, $\mathrm{p}=0.509$ ). Additionally, the diameter of bile ducts was not correlated with the DH and KGR $(\rho=-0.396, p=0.084 ; \rho=-0.269, p=0.252$, respectively). In the CRLM group, the $\mathrm{DH}$ and $\mathrm{KGR}$ were similar in patients with cirrhosis or liver fibrosis $(n=6)$ and those with normal background liver $(n=18)$ (6.4 [3.09.3] versus 4.6 [3.0-6.4] \%, respectively, $p=0.205$; KGR: 1.8 [0.9-2.6] versus 1.2 [0.8$2.1] \% /$ week, respectively, $p=0.351$ ).

Table 3. Assessment of FLR hypertrophy after PVE in pCCA patients with and without hyperbilirubinemia.

\begin{tabular}{|c|c|c|c|}
\hline Variable & $\begin{array}{l}\text { Hyperbilirubinemia } \\
\text { group }(n=10)\end{array}$ & $\begin{array}{l}\text { Without hyperbilirubinemia } \\
\text { group }(n=9)\end{array}$ & $p$ value \\
\hline Age (years) & 70 [67-72] & 70 [64-76] & 0.563 \\
\hline Gender & & & 0.650 \\
\hline Female & $3(30 \%)$ & $4(44 \%)$ & \\
\hline Male & $7(70 \%)$ & $5(56 \%)$ & \\
\hline BMI $\left(\mathrm{kg} / \mathrm{m}^{2}\right)$ & $22.8[22.0-26.1]$ & $25.6[23.2-27.0]$ & 0.102 \\
\hline Charlson comorbidity index & $6[5-8]$ & $7[4-8]$ & 0.934 \\
\hline Cholangitis & $2(20 \%)$ & $1(11 \%)$ & 1.000 \\
\hline \multicolumn{4}{|l|}{ Serum biochemistry before PVE } \\
\hline Bilirubin $(\mu \mathrm{mol} / \mathrm{L})$ & 63.9 [56.4-95.5] & $25.8[10.0-39.4]$ & $<0.001$ \\
\hline GGT (U/L) & 535 [333-1029] & 339 [106-753] & 0.191 \\
\hline ALP (U/L) & 369 [271-725] & $255[128-420]$ & 0.083 \\
\hline $\mathrm{CRP}(\mathrm{mg} / \mathrm{L})$ & $42.1[16.4-94.7]$ & $20.6[7.0-89.0]$ & 0.637 \\
\hline Number of days after PVE & $22[16-26]$ & 20 [17-22] & 0.652 \\
\hline FLR increase (mL) & $131[68-189]$ & $91[28-144]$ & 0.253 \\
\hline FLR increase per day (mL/day) & $6.2[2.2-11.0]$ & $4.1[1.5-7.2]$ & 0.288 \\
\hline \%Hypertrophy & $56[15-67]$ & $30[8-51]$ & 0.191 \\
\hline \%Hypertrophy per week & $16.4[6.0-27.0]$ & $10.4[2.8-16.1]$ & 0.221 \\
\hline $\mathrm{DH}(\%)$ & $5.6[3.0-7.5]$ & $5.7[2.4-7.0]$ & 0.806 \\
\hline KGR (\% per week) & $1.7[1.0-2.4]$ & $1.9[0.8-2.4]$ & 1.000 \\
\hline
\end{tabular}

Data are presented as median [interquartile range]. pCCA, perihilar cholangiocarcinoma; PVE, portal vein embolization; FLR, future liver remnant; GGT, gamma-glutamyl transferase; ALP, alkaline phosphatase; CRP, C-reactive protein; DH, Degree of hypertrophy; KGR, kinetic growth rate.

The FLR hypertrophy response was analyzed according to the side (unilateral, bilateral) of biliary drainage prior to PVE in seventeen patients with pCCA. Patients with unilateral drainage $(n=11)$ had a higher $\mathrm{DH}$ than those with bilateral drainage $(n=6)$ (6.7 [4.9-7.9] versus 2.7 [1.5-4.2] \%, $p=0.012$ ) (Fig. 2). The characteristics of $p C C A$ 
patients with unilateral and bilateral biliary drainage were similar (Supplementary Table 3).

A

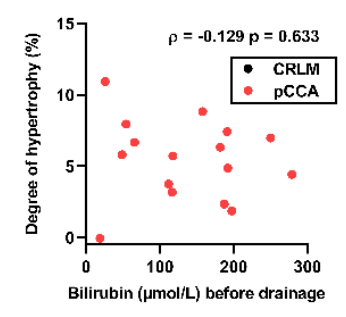

C

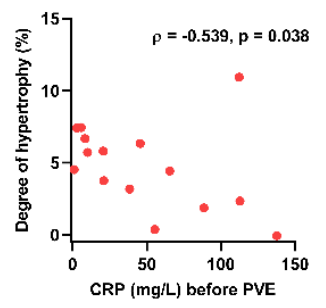

E

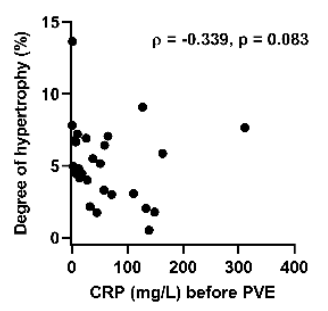

B

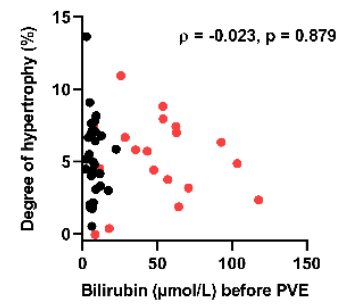

D

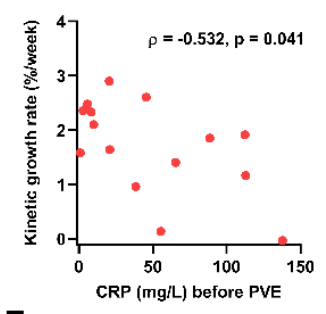

$\mathbf{F}$

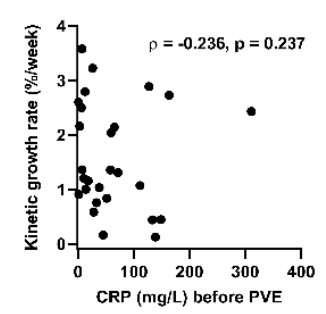

Figure 1. Serum CRP negatively correlates with hypertrophy of FLR in patients with pCCA. Correlations between degree of hypertrophy of FLR and serum bilirubin levels before drainage (A) and before PVE (B). Correlations between serum CRP levels and degree of hypertrophy and kinetic growth rate of FLR in patients with PCCA ( $C$ and $\mathbf{D}$, respectively) and CRLM ( $E$ and $F$, respectively). Correlations were assessed using Spearman's rank test. CRLM, colorectal liver metastasis; pCCA, perihilar cholangiocarcinoma; CRP, C-reactive protein.

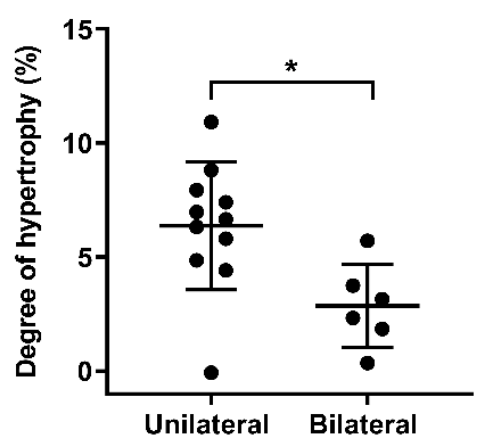

Figure 2. Degree of hypertrophy of the future liver remnant is higher in patients with unilateral biliary drainage. Seventeen out of nineteen patients with perihilar cholangiocarcinoma received biliary drainage for relief of cholestasis prior to portal vein embolization. The future liver remnant (FLR) was 
drained in patients receiving unilateral drainage $(n=11)$. Mann-Whitney $U$ test was used to compared degree of hypertrophy of FLR between two groups. Asterisks indicate significance level: ${ }^{*} p<0.05$.

\section{Correlations between routine blood tests and FLR hypertrophy}

A Spearman correlation analysis was performed to evaluate associations between serum biochemistry and FLR hypertrophy parameters. Serum CRP levels before PVE were negatively correlated with the $\mathrm{DH}(\rho=-0.401, p=0.009)$, and showed a strong tendency to be negatively correlated with $\operatorname{KGR}(\rho=-0.300, p=0.054)$. Subgroup analysis revealed that the inverse relationships between CRP and FLR hypertrophy were apparent in patients with pCCA (DH: $\rho=-0.539, p=0.038$; KGR: $\rho=-0.532, p=0.041$, respectively) (Fig. 1C-D), but not in patients with CRLM (DH: $\rho=-0.339, p=0.083$; KGR: $\rho=-0.236, p=0.237$, respectively) (Fig. 1E-F). Additionally, serum albumin levels before PVE were positively correlated with the $\mathrm{DH}(\rho=+0.301, p=0.045)$. All correlations between serum biochemistry parameters and FLR hypertrophy are summarized in Supplementary Table 4.

\section{Discussion}

This study has evaluated whether cholestasis affects PVE-induced hypertrophy of the future liver remnant. The main findings are that the degree of hypertrophy and kinetic growth rate of the FLR are similar in patients with CRLM and PCCA, and in PCCA patients with or without hyperbilirubinemia. Neither serum bilirubin levels before drainage, nor before PVE, were correlated with indices of FLR hypertrophy. Patients with $\mathrm{PCCA}$ receiving unilateral biliary drainage of the FLR had a higher $\mathrm{DH}$ than those with bilateral drainage. Elevated inflammatory response was associated with impaired liver growth in patients with pCCA.

In previous studies, cholestasis has been associated with poor regenerative capacity of the liver after partial hepatectomy, with toxicity due to bile salt overload in hepatocytes serving as an underlying mechanism 20. Patients with pCCA, characterized by cholestasis in one or both liver halves due to biliary obstruction, have been shown to have a high 90-day mortality rate of up to $14 \%$ after partial liver resection, despite routine biliary decompression prior to surgery ${ }^{21}$. In the present study, we observed similar FLR hypertrophy after PVE, in patients with CRLM and PCCA, 
which is in line with recent findings elsewhere ${ }^{22}$. The majority (89\%) of pCCA patients here received drainage prior to PVE. Nine out of nineteen patients with pCCA had serum bilirubin levels lower than $50 \mu \mathrm{mol} / \mathrm{L}$ at the time of PVE. These nine patients had comparable DH and KGR with pCCA patients who had bilirubin levels higher than $50 \mu \mathrm{mol} / \mathrm{L}$. Moreover, serum bilirubin either before drainage or before PVE were not correlated with the DH and KGR. The similar FLR hypertrophy in cholestatic and noncholestatic patients suggests that the growth-stimulating effect of PVE outweighs any potential negative effects of bile salt accumulation.

Biliary drainage is widely used to decompress the biliary tree and improve cholestatic status before liver resection ${ }^{23,24}$. In our study, seventeen of nineteen patients with pCCA underwent biliary drainage before PVE. Remarkably, the DH was higher in those patients with unilateral drainage of the FLR than in those with bilateral drainage. Our findings are thus consistent with the study of Ishizawa et al., who observed a higher hypertrophy ratio and bilirubin clearance rate of the FLR after unilateral drainage of the FLR group than the bilateral drainage group ${ }^{25}$. In our cohort, patients with bilateral drainage had insufficient improvement of cholestasis upon initial biliary drainage. Of note, the occurrence of cholangitis appeared to be higher in the bilateral drainage group ( 2 out $6=33 \%$ versus 1 out of $11=9 \%$ ), although low event rates do not allow statistical back-up. It is nonetheless tempting to speculate that cholangitis and associated inflammation contributed to impaired liver hypertrophy in bilaterally drained patients. It should be noted that serum CRP, which was found to negatively correlate with $\mathrm{DH}$, was not affected by the side of drainage.

Our study has demonstrated a negative relation between serum CRP levels before PVE and the DH and KGR, especially in patients with pCCA. To the best of our knowledge, this is the first study to reveal the association of an inflammation marker and FLR growth in clinical patients. Notably, serum CRP levels were 1.7 fold higher after biliary drainage in patients with $\mathrm{PCCA}$, albeit this did not reach statistical significance $(p=0.167)$. The tentative CRP elevation might have been due to the retrograde route through the proximal small intestine in the case of endoscopic biliary drainage (EBD). In support of this notion, 15 out of 17 (88\%) drained patients received an EBD or EBD+PTBD (percutaneous transhepatic biliary drainage) procedure.

The question of which molecular pathway could underlie the observed relationship between CRP and FRL hypertrophy remains unclear. However, it is known that 
cytokines (e.g. interleukin-8) induced by inflammation can stimulate hepatic infiltration of neutrophils ${ }^{26}$. As part of innate immune defenses, neutrophils produce reactive oxygen species and hypochlorous acid that cause cytotoxicity ${ }^{27}$. Experimental and human studies have shown that periportal neutrophil infiltration is positively correlated with the degree of liver damage ${ }^{27,28}$, which is recognised as having a negative impact on liver hypertrophy. Serum CRP alterations may have promise as a surrogate marker of PVE-induced FLR hypertrophy in patients with pCCA.

Certain limitations have to be acknowledged. For example, the study's retrospective design makes it impossible to avoid selection bias when including patients. In addition, the sample size of the present cohort is rather small, which means some events appear rare, such as cholangitis, PTBD procedure, and non-drainage of the liver in pCCA. As such, it is impossible to conduct a logistic regression to assess the predictive value of these factors on FLR hypertrophy. Furthermore, nine patients had small metastases in segment II or III, which might have impacted PVE-induced hypertrophy. Nevertheless, the volume of the small metastases was excluded when analyzing FLR volumes.

Besides bilirubin, bile salts are another indicator reflecting cholestasis, although not one routinely assayed in clinical chemistry. Due to the unavailability of serum samples, bile salt levels could not be assessed. Bile salts are involved in liver regeneration after partial hepatectomy via activation of the nuclear bile salt receptor FXR ${ }^{16}$. FXR plays a central role in maintaining bile salt homeostasis, and regulating hepatocyte cell cycle progression 17. Moreover, we have previously demonstrated that FXR agonist obeticholic acid accelerates liver growth following PVE in rabbits ${ }^{13}$. Bile salt levels at $5 \mathrm{~h}$ after PVE predict FLR volume after 3 weeks in patients scheduled for resection ${ }^{12}$. It would therefore be worthwhile including serum bile salt measurements in larger prospective studies to address the influence of cholestasis on PVE-induced hypertrophy in pCCA patients.

In conclusion, cholestasis was found to have no influence on hypertrophy of the FLR in patients undergoing PVE. And, unilateral biliary drainage of FLR appears to induce more FLR hypertrophy than bilateral drainage. Systematic CRP changes may thus be promising to predict FLR hypertrophy in patients with pCCA.

\section{Acknowledgements}


Chapter 5

We are grateful to Lloyd Brandts from the Department of Clinical Epidemiology and Medical Technology Assessment of Maastricht University Medical Center for statistical advice. 


\section{References}

1. Ribero D, Abdalla EK, Madoff DC, Donadon M, Loyer EM, Vauthey JN. Portal vein embolization before major hepatectomy and its effects on regeneration, resectability and outcome. Br J Surg 2007;94:1386-94.

2. Olthof PB, Aldrighetti L, Alikhanov R, Cescon M, Groot Koerkamp B, Jarnagin WR et al. Portal Vein Embolization is Associated with Reduced Liver Failure and Mortality in High-Risk Resections for Perihilar Cholangiocarcinoma. Ann Surg Oncol 2020;27:2311-8.

3. Clavien PA, Petrowsky H, DeOliveira ML, Graf R. Strategies for safer liver surgery and partial liver transplantation. N Engl J Med 2007;356:1545-59.

4. Ferrero A, Viganò L, Polastri R, Muratore A, Eminefendic H, Regge D et al. Postoperative liver dysfunction and future remnant liver: where is the limit? Results of a prospective study. World $J$ Surg 2007;31:1643-51.

5. Chapelle T, Op De Beeck B, Huyghe I, Francque S, Driessen A, Roeyen G et al. Future remnant liver function estimated by combining liver volumetry on magnetic resonance imaging with total liver function on $(99 \mathrm{~m})$ Tc-mebrofenin hepatobiliary scintigraphy: can this tool predict post-hepatectomy liver failure? HPB (Oxford) 2016;18:494-503.

6. Denys A, Prior J, Bize P, Duran R, De Baere T, Halkic N et al. Portal vein embolization: what do we know? Cardiovasc Intervent Radiol 2012;35:999-1008.

7. Liu H, Zhu S. Present status and future perspectives of preoperative portal vein embolization. Am J Surg 2009;197:686-90.

8. Kasai Y, Hatano E, Iguchi K, Seo S, Taura K, Yasuchika K et al. Prediction of the remnant liver hypertrophy ratio after preoperative portal vein embolization. Eur Surg Res 2013;51:129-37.

9. Olthof PB, Wiggers JK, Groot Koerkamp B, Coelen RJ, Allen PJ, Besselink MG et al. Postoperative Liver Failure Risk Score: Identifying Patients with Resectable Perihilar Cholangiocarcinoma Who Can Benefit from Portal Vein Embolization. J Am Coll Surg 2017;225:387-94.

10. Hasselgren K, Sandström P, Røsok BI, Sparrelid E, Lindell G, Larsen PN et al. Future Liver Remnant (FLR) Increase in Patients with Colorectal Liver Metastases Is Highest the First Week After Portal Vein Occlusion : FLR Increase in Patients with CRLM Is Highest the First Week After PVO. J Gastrointest Surg 2019;23:556-62.

11. Heil J, Korenblik R, Heid F, Bechstein WO, Bemelmans M, Binkert C et al. Preoperative portal vein or portal and hepatic vein embolization: DRAGON collaborative group analysis. Br J Surg 2021;108:834-42.

12. Hoekstra LT, van Lienden KP, Schaap FG, Chamuleau RA, Bennink RJ, van Gulik TM. Can plasma bile salt, triglycerides, and apoA-V levels predict liver regeneration? World J Surg 2012;36:2901-8.

13. Olthof PB, Huisman F, Schaap FG, van Lienden KP, Bennink RJ, van Golen RF et al. Effect of obeticholic acid on liver regeneration following portal vein embolization in an experimental model. Br J Surg 2017;104:590-9.

14. van Golen RF, Olthof PB, Lionarons DA, Reiniers MJ, Alles LK, Uz Z et al. FXR agonist obeticholic acid induces liver growth but exacerbates biliary injury in rats with obstructive cholestasis. Sci Rep 2018;8:16529.

15. van de Laarschot LF, Jansen PL, Schaap FG, Olde Damink SW. The role of bile salts in liver regeneration. Hepatol Int 2016;10:733-40. 


\section{Chapter 5}

16. Huang W, Ma K, Zhang J, Qatanani M, Cuvillier J, Liu J et al. Nuclear receptor-dependent bile acid signaling is required for normal liver regeneration. Science 2006;312:233-6.

17. Schaap FG, Trauner M, Jansen PL. Bile acid receptors as targets for drug development. Nat Rev Gastroenterol Hepatol 2014;11:55-67.

18. de Haan L, van der Lely SJ, Warps AK, Hofsink Q, Olthof PB, de Keijzer MJ et al. Posthepatectomy liver regeneration in the context of bile acid homeostasis and the gut-liver signaling axis. J Clin Transl Res 2018;4:1-46.

19. Yim J, Hyun D, Cho SK, Park KB, Park HS, Shin SW et al. Effect of Hyperbilirubinemia on Hepatic Hypertrophy after Portal Vein Embolization and Liver Failure after Hepatectomy in Primary Biliary Malignancy. J Vasc Interv Radiol 2019;30:31-7.

20. Forbes SJ, Newsome PN. Liver regeneration - mechanisms and models to clinical application. Nat Rev Gastroenterol Hepatol 2016;13:473-85.

21. Wiggers JK, Groot Koerkamp B, Cieslak KP, Doussot A, van Klaveren D, Allen PJ et al. Postoperative Mortality after Liver Resection for Perihilar Cholangiocarcinoma: Development of a Risk Score and Importance of Biliary Drainage of the Future Liver Remnant. J Am Coll Surg 2016;223:321-31.e1.

22. de Haan LR, Verheij J, van Golen RF, Horneffer-van der Sluis V, Lewis MR, Beuers UHW et al. Unaltered Liver Regeneration in Post-Cholestatic Rats Treated with the FXR Agonist Obeticholic Acid. Biomolecules 2021;11:260.

23. Inamdar S, Slattery E, Bhalla R, Sejpal DV, Trindade AJ. Comparison of Adverse Events for Endoscopic vs Percutaneous Biliary Drainage in the Treatment of Malignant Biliary Tract Obstruction in an Inpatient National Cohort. JAMA Oncol 2016;2:112-7.

24. Lidsky ME, Jarnagin WR. Surgical management of hilar cholangiocarcinoma at Memorial Sloan Kettering Cancer Center. Ann Gastroenterol Surg 2018;2:304-12.

25. Ishizawa T, Hasegawa K, Sano K, Imamura H, Kokudo N, Makuuchi M. Selective versus total biliary drainage for obstructive jaundice caused by a hepatobiliary malignancy. Am J Surg 2007;193:149-54.

26. Van Sweringen HL, Sakai N, Quillin RC, Bailey J, Schuster R, Blanchard J et al. Roles of hepatocyte and myeloid CXC chemokine receptor-2 in liver recovery and regeneration after ischemia/reperfusion in mice. Hepatology 2013;57:331-8.

27. Li M, Cai SY, Boyer JL. Mechanisms of bile acid mediated inflammation in the liver. Mol Aspects Med 2017;56:45-53.

28. Cai SY, Ouyang X, Chen Y, Soroka CJ, Wang J, Mennone A et al. Bile acids initiate cholestatic liver injury by triggering a hepatocyte-specific inflammatory response. JCI Insight 2017;2:e90780. 
Supplementary Table 1. Serum biochemistry tests in patients with pCCA and CRLM.

\begin{tabular}{|c|c|c|c|c|c|}
\hline \multirow{2}{*}{ Parameters } & \multicolumn{2}{|c|}{$\operatorname{pCCA}(n=19)$} & \multirow{2}{*}{$p$ value ${ }^{a}$} & \multirow{2}{*}{$\begin{array}{c}\text { CRLM }(n=31) \\
\text { Before PVE }\end{array}$} & \multirow{2}{*}{$p$ value ${ }^{b}$} \\
\hline & Before drainage & Before PVE & & & \\
\hline Bilirubin $(\mu \mathrm{mol} / \mathrm{L})$ & $137.8[57.0-191.9]$ & 53.8 [25.8-64.5] & 0.001 & $6.9[5.2-8.9]$ & $<0.001$ \\
\hline Albumin (g/L) & 36.3 [33.3-40.8] & 35.0 [27.7-37.3] & 0.188 & 36.0 [30.3-42.0] & 0.246 \\
\hline INR & $1.02[0.98-1.12]$ & $1.02[1.00-1.08]$ & 0.364 & $1.01[0.97-1.10]$ & 0.732 \\
\hline GGT (U/L) & 735 [297-1048] & 434 [225-878] & 0.300 & 77 [36-185] & $<0.001$ \\
\hline ALP (U/L) & 438 [249-571] & 318 [208-463] & 0.173 & 121 [86-155] & $<0.001$ \\
\hline ALT (U/L) & 133 [76-310] & 103 [58-127] & 0.026 & 50 [27-150] & 0.235 \\
\hline AST (U/L) & 124 [78-169] & 74 [42-103] & 0.087 & 39 [26-94] & 0.053 \\
\hline CRP (mg/L) & 22.7 [11.2-73.8] & 38.5 [8.2-88.6] & 0.131 & $38.0[10.1-111.0]$ & 0.684 \\
\hline WBC count $\left(10^{9} / \mathrm{L}\right)$ & $8.7[5.3-11.1]$ & $8.2[6.0-10.0]$ & 0.155 & $6.5[6.2-8.9]$ & 0.271 \\
\hline
\end{tabular}

Data are presented as median [interquartile range].

a $p$ values were calculated for comparison between "before drainage" and "before PVE" values, within patients with pCCA.

b $p$ values were calculated for comparison of "before PVE" values between pCCA and CRLM groups.

pCCA, perihilar cholangiocarcinoma; CRLM, colorectal liver metastasis; INR, international normalized ratio; GGT, gamma-glutamyl transferase; ALP, alkaline phosphatase; ALT, alanine aminotransferase; AST, aspartate aminotransferase; CRP, C-reactive protein; WBC, white blood cell.

Supplementary Table 2. Characteristics of 10 matched patients with CRLM and pCCA.

\begin{tabular}{lccc}
\hline Characteristics & CRLM $(\mathrm{n}=10)$ & pCCA $(\mathrm{n}=10)$ & $\mathrm{p}$ value \\
\hline Age (years) & $61[47-71]$ & $70[69-73]$ & 0.079 \\
Gender & & & 1.000 \\
$\quad$ Female & $4(40 \%)$ & $5(50 \%)$ & \\
$\quad$ Male & $6(60 \%)$ & $5(50 \%)$ & \\
BMI $\left(\mathrm{kg} / \mathrm{m}^{2}\right)$ & $24.6[23.1-29.0]$ & $25.7[23.1-26.5]$ & 0.705 \\
Diabetes & $2(20 \%)$ & $2(20 \%)$ & 1.000 \\
Charlson comorbidity index & $8[6-9]$ & $8[7-9]$ & 0.969 \\
Cirrhosis & $0(0 \%)$ & $0(0 \%)$ & 1.000 \\
\hline
\end{tabular}

Data are presented as median [interquartile range] or frequency (percentage). CRLM, colorectal liver metastasis; pCCA, perihilar cholangiocarcinoma; BMI, body mass index. 
Chapter 5

Supplementary Table 3. Characteristics of 17 pCCA patients with biliary drainage.

\begin{tabular}{|c|c|c|c|}
\hline Variable & Unilateral drainage $(n=11)$ & Bilateral drainage $(n=6)$ & $p$ value \\
\hline Age (years) & 70 [67-72] & 73 [69-77] & 0.359 \\
\hline Gender & & & 1.000 \\
\hline Female & $5(46 \%)$ & $2(33 \%)$ & \\
\hline Male & $6(54 \%)$ & $4(67 \%)$ & \\
\hline BMI $\left(\mathrm{kg} / \mathrm{m}^{2}\right)$ & $24.6[22.6-27.0]$ & $22.8[22.1-27.8]$ & 0.615 \\
\hline Diabetes & $4(36 \%)$ & $0(0 \%)$ & 0.237 \\
\hline Charlson comorbidity index & $6[5-8]$ & $7[5-9]$ & 0.721 \\
\hline Cholangitis & $1(9 \%)$ & $2(33 \%)$ & 0.515 \\
\hline Bismuth-Corlette classification & & & 0.266 \\
\hline I & $1(9 \%)$ & $0(0 \%)$ & \\
\hline II & $2(18 \%)$ & $0(0 \%)$ & \\
\hline IIIA & $3(27 \%)$ & $1(17 \%)$ & \\
\hline IIIB & $1(9 \%)$ & $0(0 \%)$ & \\
\hline IV & $4(37 \%)$ & $5(83 \%)$ & \\
\hline \multicolumn{4}{|c|}{$\begin{array}{l}\text { Serum biochemistry tests before } \\
\text { PVE }\end{array}$} \\
\hline Bilirubin $(\mu \mathrm{mol} / \mathrm{L})$ & 53.8 [28.6-62.9] & $60.8[36.9-82.7]$ & 0.421 \\
\hline Albumin $(\mathrm{g} / \mathrm{L})$ & $35.5[30.0-38.5]$ & $30.5[22.4-35.3]$ & 0.158 \\
\hline INR & $1.01[0.95-1.12]$ & $1.02[1.01-1.09]$ & 0.359 \\
\hline GGT (U/L) & 719 [414-1083] & 342 [281-612] & 0.108 \\
\hline ALP (U/L) & 379 [220-662] & $291[242-376]$ & 0.396 \\
\hline ALT (U/L) & $115[60-152]$ & 66 [46-98] & 0.090 \\
\hline AST (U/L) & 82 [42-205] & 84 [46-103] & 0.914 \\
\hline CRP (mg/L) & $45.6[8.2-112.5]$ & $47.0[18.2-94.7]$ & 0.775 \\
\hline WBC count $\left(10^{9} / \mathrm{L}\right)$ & $8.8[6.2-12.5]$ & $7.1[4.9-9.3]$ & 0.289 \\
\hline Number of days after PVE & 22 [17-35] & $17[12-20]$ & 0.086 \\
\hline
\end{tabular}

Data are presented as median [interquartile range] or frequency (percentage). pCCA, perihilar cholangiocarcinoma; BMI, body mass index; PVE, portal vein embolization; INR, international normalized ratio; GGT, gamma-glutamyl transferase; ALP, alkaline phosphatase; ALT, alanine aminotransferase; AST, aspartate aminotransferase; CRP, C-reactive protein; WBC, white blood cell. 
Cholestasis and PVE-induced FLR hypertrophy

Supplementary Table 4. Correlations between serum biochemistry parameters and FLR hypertrophy.

\begin{tabular}{|c|c|c|c|c|}
\hline \multirow{2}{*}{ Parameters } & \multicolumn{2}{|c|}{$\mathrm{DH}$} & \multicolumn{2}{|c|}{ KGR } \\
\hline & $\rho$ & $\mathrm{p}$ value & $\rho$ & $\mathrm{p}$ value \\
\hline \multicolumn{5}{|l|}{ Entire cohort $(n=50)$} \\
\hline \multicolumn{5}{|c|}{ Parameters before PVE } \\
\hline Bilirubin $(\mu \mathrm{mol} / \mathrm{L})$ & -0.023 & 0.879 & 0.148 & 0.321 \\
\hline Albumin (g/L) & 0.301 & 0.045 & 0.110 & 0.471 \\
\hline INR & -0.189 & 0.193 & -0.120 & 0.412 \\
\hline GGT (U/L) & 0.139 & 0.345 & 0.074 & 0.618 \\
\hline ALP (U/L) & -0.021 & 0.892 & -0.007 & 0.963 \\
\hline ALT (U/L) & -0.018 & 0.911 & -0.154 & 0.342 \\
\hline AST (U/L) & -0.120 & 0.442 & -0.039 & 0.803 \\
\hline $\mathrm{CRP}(\mathrm{mg} / \mathrm{L})$ & -0.401 & 0.009 & -0.300 & 0.054 \\
\hline WBC count $\left(10^{9} / L\right)$ & -0.072 & 0.640 & -0.083 & 0.589 \\
\hline \multicolumn{5}{|c|}{ CRLM patients $(n=31)$} \\
\hline \multicolumn{5}{|c|}{ Parameters before PVE } \\
\hline Bilirubin $(\mu \mathrm{mol} / \mathrm{L})$ & -0.082 & 0.678 & 0.215 & 0.273 \\
\hline Albumin (g/L) & 0.176 & 0.370 & 0.066 & 0.738 \\
\hline INR & 0.013 & 0.945 & 0.034 & 0.855 \\
\hline GGT (U/L) & 0.113 & 0.560 & 0.116 & 0.548 \\
\hline ALP (U/L) & -0.275 & 0.164 & -0.130 & 0.519 \\
\hline $\mathrm{ALT}(\mathrm{U} / \mathrm{L})$ & -0.312 & 0.129 & -0.329 & 0.108 \\
\hline AST (U/L) & -0.304 & 0.140 & -0.077 & 0.713 \\
\hline CRP (mg/L) & -0.339 & 0.083 & -0.236 & 0.237 \\
\hline WBC count $\left(10^{9} / L\right)$ & -0.151 & 0.443 & -0.108 & 0.584 \\
\hline \multicolumn{5}{|l|}{ pCCA patients $(n=19)$} \\
\hline \multicolumn{5}{|c|}{ Parameters before drainage } \\
\hline Bilirubin $(\mu \mathrm{mol} / \mathrm{L})$ & -0.129 & 0.633 & 0.097 & 0.721 \\
\hline Albumin (g/L) & 0.302 & 0.340 & 0.256 & 0.422 \\
\hline INR & -0.502 & 0.057 & -0.203 & 0.469 \\
\hline GGT (U/L) & 0.565 & 0.023 & -0.032 & 0.905 \\
\hline ALP (U/L) & 0.391 & 0.134 & 0.256 & 0.339 \\
\hline $\mathrm{ALT}(\mathrm{U} / \mathrm{L})$ & 0.289 & 0.296 & -0.021 & 0.940 \\
\hline AST (U/L) & 0.407 & 0.149 & -0.086 & 0.771 \\
\hline $\mathrm{CRP}(\mathrm{mg} / \mathrm{L})$ & -0.218 & 0.455 & -0.345 & 0.227 \\
\hline WBC count $\left(10^{9} / L\right)$ & -0.135 & 0.661 & -0.030 & 0.922 \\
\hline \multicolumn{5}{|c|}{ Parameters before PVE } \\
\hline Bilirubin $(\mu \mathrm{mol} / \mathrm{L})$ & -0.105 & 0.668 & -0.025 & 0.920 \\
\hline Albumin (g/L) & 0.503 & 0.039 & 0.415 & 0.098 \\
\hline INR & -0.513 & 0.030 & -0.469 & 0.050 \\
\hline GGT (U/L) & 0.481 & 0.037 & -0.016 & 0.949 \\
\hline ALP (U/L) & 0.353 & 0.165 & 0.172 & 0.510 \\
\hline ALT (U/L) & 0.579 & 0.024 & 0.271 & 0.328 \\
\hline AST (U/L) & 0.298 & 0.229 & 0.037 & 0.884 \\
\hline $\mathrm{CRP}(\mathrm{mg} / \mathrm{L})$ & -0.539 & 0.038 & -0.532 & 0.041 \\
\hline WBC count $\left(10^{9} / \mathrm{L}\right)$ & 0.032 & 0.903 & -0.225 & 0.384 \\
\hline
\end{tabular}

Spearman correlation analyses were performed to evaluate associations between serum biochemistry parameters and FLR hypertrophy. DH, Degree of hypertrophy; KGR, kinetic growth rate; INR, international normalized ratio; GGT, gamma-glutamyl transferase; ALP, alkaline phosphatase; ALT, alanine 


\section{Chapter 5}

aminotransferase; AST, aspartate aminotransferase; CRP, C-reactive protein; WBC, white blood cell; CRLM, colorectal liver metastasis; pCCA, perihilar cholangiocarcinoma.

A

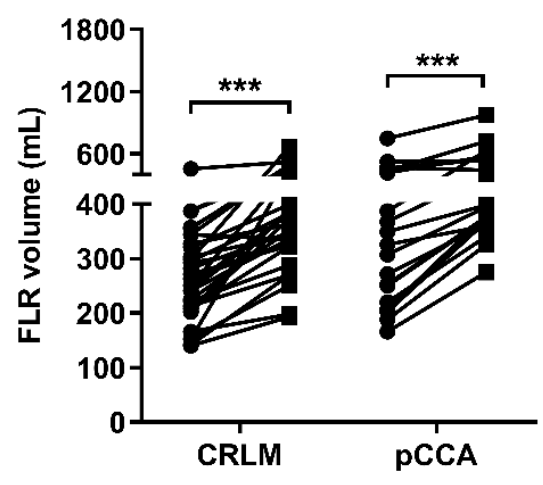

B

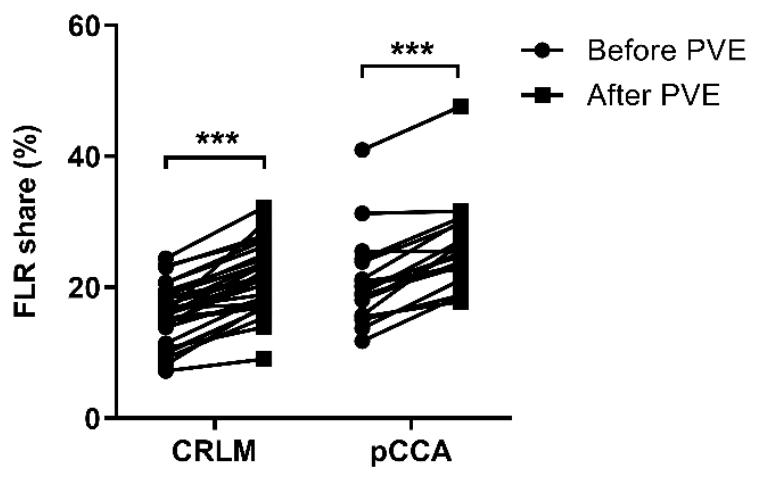

Supplementary Figure 1. Volumetric expansion of the future liver remnant by PVE in individual patients. The increase of absolute future liver remnant (FLR) volume (A) and FLR share (B) after portal vein embolization (PVE) in patients with colorectal liver metastasis (CRLM) and perihilar cholangiocarcinoma ( $\mathrm{pCCA}$ ). Wilcoxon signed rank test was used to compared FLR volume between before and after PVE in each group of patients. Asterisks indicate significance level: ${ }^{* \star} p<0.001$. 




\section{Part III}

Restored bile salt-FGF19 signaling by chyme reinfusion in intestinal failure 



\section{Chapter 6}

\section{Chyme reinfusion restores the regulatory bile salt-FGF19 axis in intestinal failure patients}

Kiran V.K. Koelfat*, Denis Picot*, Xinwei Chang*, Mireille Desille, Hans M. van Eijk, Sander M.J. van Kuijk, Martin Lenicek, Sabrina Layec, Marie Carsin, Laurence Dussaulx, Eloi Seynhaeve, Florence Trivin, Laurence Lacaze, Ronan Thibault**, Frank G. Schaap** and Steven W.M. Olde Damink** *Authors share co-first authorship, ${ }^{* *}$ Authors share co-senior authorship. 


\section{Abstract}

Introduction: Automated chyme reinfusion (CR) in intestinal failure (IF) patients with a temporary double enterostomy (TDE) restores intestinal function and protects against liver injury, but the mechanisms are incompletely understood. The aim was to investigate whether beneficial effects of $C R$ relate to functional recovery of enterohepatic signaling via the bile salt-FGF19 axis.

Methods: Blood samples were collected from 12 patients, 3 days before, at start, and 1, 3, 5 and 7 weeks after CR initiation. Plasma FGF19, total bile salts (TBS), C4 (marker of bile salt synthesis), citrulline (CIT), bile salt composition, liver tests and nutritional risk indices were determined. Paired small bowel biopsies prior to CR and after 21 days were taken and genes related to bile salt homeostasis and enterocyte function were assessed.

Results: CR induced an increase in plasma FGF19 and decreased C4 levels, indicating restored regulation of bile salt synthesis via endocrine FGF19 action. TBS remained unaltered during CR. Intestinal FXR was upregulated after 21 days of CR. Secondary and deconjugated bile salt fractions were increased after $\mathrm{CR}$, reflecting restored microbial metabolism of host bile salts. Furthermore, CIT and albumin levels were gradually rising after $C R$, while abnormal serum liver tests normalized after $C R$, indicating restored intestinal function, improved nutritional status and amelioration of liver injury. CR increased gene transcripts related to enterocyte number, carbohydrate handling and bile salt homeostasis. Finally, the reciprocal FGF19/C4 response after 7 days, predicted the plasma CIT time course.

Conclusions: CR in IF-TDE patients restored bile salt-FGF19 signaling and improved gut-liver function. Beneficial effects of CR are partly mediated by recovery of the bile salt-FGF19 axis and subsequent homeostatic regulation of bile salt synthesis. 


\section{Introduction}

A temporary double enterostomy (TDE) is most often created to avoid or protect high risk anastomoses in frail or sick patients. TDEs are often used in the acute setting of intestinal ischaemia resulting from e.g. a thrombo-embolic event or herniation. Unfortunately, some patients may develop intestinal failure (IF) due to (functional) short bowel syndrome, requiring parenteral nutrition (PN) and/or intravenous fluid supplementation (IVS) until recovery for surgical re-establishment of intestinal continuity, typically 3 to 6 months after primary surgery ${ }^{1}$. Prolonged PN dependence is often necessary, but is associated with risk of increased morbidity and mortality 2, 3 , mainly related to central venous tract complications, catheter-related infections, central venous thrombosis, or related to catheter replacement. The development of IFassociated liver disease (IFALD) frequently occurs ${ }^{1}$. An alternative treatment is chyme (intestinal or fistula secretions) reinfusion (CR), through an extracorporeal enteral nutrition technique, into the distal part of the small bowel. This method results in improved nutritional status, better intestinal absorptive function, recovery from liver disease, reduced intestinal secretions and shorter period to PN independence in retrospective studies ${ }^{4-7}$. The mechanisms underlying the beneficial effects of chyme reinfusion are incompletely understood, but functional recovery of the enterohepatic circulation of bile salts and attendant signaling has been postulated ${ }^{4}$.

Fibroblast growth factor (FGF19) is a postprandial hormone secreted by ileocytes in a bile salt-stimulated fashion that involves activation of the bile salt-sensing transcription factor Farnesoid X Receptor $(F X R)^{8}$. The key role of FGF19 is to control bile salt homeostasis by negatively regulating bile salt synthesis via repression of the bile saltsynthetic enzyme cholesterol-7 $\alpha$-hydroxylase (CYP7A1) in the liver ${ }^{8}$. Disruption of the negative feedback loop results in unopposed bile salt synthesis. As a result of low circulating FGF19 levels, bile salt overproduction occurs and this has been associated with pediatric and adult IFALD, or primary bile acid diarrhea ${ }^{9-15}$. Currently, pharmacological treatment with FGF19 analogues in patients with hepatobiliary diseases including primary biliary cholangitis or non-alcoholic steatohepatitis has been evaluated clinically and renders promising therapeutic effects ${ }^{16,17}$.

In the RESCUE study presented here, we test the hypothesis that functional recovery of the enterohepatic circulation by $\mathrm{CR}$ and attendant restoration of the regulatory bile salt-FGF19 axis in IF patients with a TDE, is associated with CR-related beneficial 
effects.

\section{Methods}

\section{Study design and participants}

The RESCUE study (restored enterohepatic signaling: chyme reinfusion therapy) is a prospective study with a pre- and post-intervention design conducted at Rennes University Hospital (CHU Rennes) and Clinique Saint Yves, Rennes, France (a tertiary referral center for intestinal rehabilitation of patients prior to restorative surgery) from January 2017 to July 2018. The study was registered at clinicaltrials.gov (NCT02990195). Eligible participants were adult IF patients aged 18 years or older with a temporary double enterostomy intended to receive CR as primary care. Further inclusion criteria were a minimum of $25 \mathrm{~cm}$ of healthy distal small intestine that was suitable for CR. We excluded patients with mental disability, pregnancy or lactation, hepatocellular carcinoma, auto-immune disorders, blood coagulation disorders, or shock of any cause. None of the patients had any known viral or genetic liver disease prior to the double enterostomy. We obtained written informed consent from all participants. This study was approved by the Institutional Review Board of Rennes University Hospital, Rennes, France (2016-A01156-45).

CR was performed at Clinique Saint Yves using the Enteromate II ${ }^{\circledR}$ system (Labodial, Les Clayes Sous Bois, France) as described previously ${ }^{18}$. In brief, proximal effluent was continuously pumped into a $30 \mathrm{~mL}$ collection container equipped with weightmonitoring. When collected volume exceeded $10 \mathrm{~mL}$, the second pump started and reinfused the collected chyme into the distal part of the small bowel. In patients with inadequate oral intake, an additional enteroclysis was applied to deliver enteral nutrition to the reinfusion tube in the efferent small bowel. CR was initiated in approximately one week after referral of the patient to Clinique Saint Yves and continuously applied for two to three months. Afterwards, patients underwent surgical re-establishment of intestinal continuity. Blood samples were collected at three days before CR (day -3), at the day of CR initiation (day 0 ), and during the course of CR (after 1, 3, 5 and 7 weeks). Patients underwent endoscopic biopsy of the distal small intestine three days before and three weeks after $\mathrm{CR}$ at $\mathrm{CHU}$ Rennes by an experienced gastroenterologist. Study design is shown in Supplementary Figure S1. 


\section{Data collection and definitions}

Data were collected and managed using a customized Access Database (Microsoft, Redmond, WA, USA). The following items were recorded: gender, age, height, body weight, body mass index (BMI), underlying diseases, afferent small intestinal length (from duodeno-jejunal flexure to the proximal stoma), efferent small intestinal length (from distal stoma to terminal part of the remnant efferent small intestine), resected length, efferent small intestinal anatomy, energy $(\mathrm{kcal} / \mathrm{kg})$ and protein $(\mathrm{g} / \mathrm{kg})$ intake (parenteral, oral, and enteral) adjusted by body weight, and duration of CR and PN. We measured proximal stoma or fecal output daily in all patients before and after CR initiation, respectively. Percent weight loss was assessed as follows: weight loss (\%) $=100^{*}$ (usual weight-present weight)/usual weight. None of the patients were diagnosed with ascites during $\mathrm{CR}$ treatment. Nutritional Risk Index (NRI) was determined as follows: $N R I=1.515^{*}$ serum albumin $(g / L)+41.77^{*}$ (present weight/usual weight) ${ }^{19}$. NRI-based risk groups were as follows: absence of malnutrition risk (NRI > 97.5), moderate malnutrition risk $(83.5 \leq \mathrm{NRI} \leq 97.5)$ and severe malnutrition risk $(\mathrm{NRI}<83.5)^{19}$.

\section{Blood samples and analyses}

Blood samples were collected from patients in the morning and after an overnight fast in three $8 \mathrm{~mL}$ vacuum tubes (serum, EDTA and lithium heparin tubes). Serum tubes were left at room temperature for $30 \mathrm{~min}$, and plasma tubes were put on ice immediately after collection. After $30 \mathrm{~min}$, all blood samples were centrifuged at 4000 rpm for $10 \mathrm{~min}$ at $4^{\circ} \mathrm{C}$. The supernatant was collected, divided into aliquots, and stored at $-80^{\circ} \mathrm{C}$ until transport to Maastricht University (Maastricht, The Netherlands) for further analysis. Plasma levels of FGF19 and pro-inflammatory cytokines (interleukin6, IL-6 and tumor necrosis factor-alpha, TNF- $\alpha$ ) were assessed by sandwich ELISA as reported previously ${ }^{20}$. Plasma citrulline (CIT, a marker of the mass of functional enterocytes) was determined by a high-performance liquid chromatography method ${ }^{6}$, 21. Plasma 7-a-hydroxy-4-cholesten-3-one ( $\mathrm{C} 4$, blood marker of bile salt synthesis) ${ }^{22}$, total bile salts (TBS), and bile salt composition (both in plasma and chyme) were assayed by liquid chromatography-mass spectrometry (Schaap and Olde Damink, manuscript in preparation). Serum liver tests (alanine aminotransferase, ALT; aspartate aminotransferase, AST; alkaline phosphatase, ALP; Y-glutamyl transferase, GGT and total bilirubin), albumin and C-reactive protein (CRP) were evaluated by routine assay 
at the Department of Clinical Biochemistry at Maastricht University Medical Center (Maastricht, The Netherlands). For baseline comparisons of TBS, FGF19 and C4 levels, own published data on twelve healthy controls (mean age 55 years, 33\% female) was used ${ }^{23}$.

\section{Gene expression analysis}

Ileal biopsies were obtained through routine endoscopic procedures and immediately preserved in RNAlater ${ }^{\circledR}$ (Thermo Scientific, Waltham, MA, USA) for 24 hours at $4^{\circ} \mathrm{C}$ and then stored at $-80^{\circ} \mathrm{C}$ (Rennes University Hospital, Rennes, France). Biopsies were ultimately transported to Maastricht University Medical Center (Maastricht, The Netherlands) for transcript analyses. Hereto, total RNA was isolated using the RNeasy Plus Mini Kit according to the manufacturer's protocol (Qiagen, Hilden, Germany). Final RNA concentration and purity were determined by spectrophotometry. A total of 750 ng RNA was converted to cDNA using SensiFAST ${ }^{\mathrm{TM}}$ CDNA Synthesis Kit (Bioline, London, UK). For the quantitative reverse transcriptase polymerase chain reaction (qRT-PCR), we used SensiFAST ${ }^{\text {TM }}$ SYBR Hi-ROX kit (Bioline, London, UK) and cDNA as template on a LightCycler ${ }^{\circledR} 480$ SW 1.5 system (Roche, Basel, Switzerland). qRTPCR data was analyzed using LinRegPCR software ${ }^{24}$, and gene expression levels were normalized to the geometric mean of 4 reference genes (36B4, PSMB4, REEP5, and HPRT1). Primer sequences provided in Supplementary Table S1. Paired ileal biopsies were available from seven of twelve patients. In remaining patients, (paired) biopsies could not be gathered for logistic reasons $(n=2)$, last-minute patient refusal $(n=1)$, or transcript data was excluded from analyses due to a non-enterocytic transcript signature $(n=2)$.

\section{Statistical analysis}

Initially, twenty patients were planned for enrollment in this study. A planned interimanalysis was performed after enrollment of two thirds of patients $(n=13)$. One patient was not included in the study after enrollment because he received enteral nutrition as enteroclysis into the downstream small bowel, before starting the protocol. On the interim-analysis ( $n=12$ ), the primary endpoint (FGF19 level) had significantly changed $(P<.005)$ justifying early termination of the study. For blood analyses, five out of seventy-two time points were missing (6.9\%). Multiple data imputation was used to handle the missing data. The mean of day -3 and day 0 measurements were taken as baseline values. Data are expressed as mean \pm standard deviation (SD), median 
(interquartile range), or frequency (percentage) where appropriate. Graphical data are expressed as mean $\pm 95 \%$ Confidence Interval $(\mathrm{Cl})$. Differences between IF patients and healthy controls were evaluated by Mann-Whitney $U$ test. Correlations were evaluated by the Pearson ( $r$ ) or Spearman's $(\rho)$ correlation coefficient dependent on data distribution. For continuous variables, changes during $\mathrm{CR}$ were evaluated by paired-samples $t$ test or Wilcoxon matched-pairs signed ranks sum test dependent on the data distribution. Categorical variables were analyzed using Fisher's exact test. To evaluate longitudinal changes, ANOVA or Friedman's test for repeated measurements, corrected using Dunn's multiple comparisons test, were used. Linear mixed models were performed to identify whether the FGF19 or C4 response predicts the evolution of markers for intestinal absorptive function (CIT) and liver injury (ALP and GGT). The Akaike's Information Criterion was used to compare the best fit of a covariance structure for the linear mixed models. $P$ value $<.05$ was considered statistically significant. SPSS statistics version 26.0 (IBM Corporation, Chicago, IL, USA) was used for all statistical analyses.

\section{Results}

\section{Patient characteristics}

Twelve patients (mean age 68 years, $42 \%$ females) were included in the study (Table 1). Despite the fact that features of IF -necessitating supplementation with $\mathrm{PN}$ - were apparent in all surgical patients, ten patients actually received PN and/or intravenous (IV) hydration at admission. Ten patients remained in the hospital during the 7 weeks course of $C R$, and two patients received home $C R$ after specific training and education. Major underlying causes for small intestine resection and/or creation of a TDE were mechanical obstruction and intestinal ischemia. Patients underwent small intestinal resection $(n=8)$ or received a terminal colostomy on the transverse $(n=3)$ or right colon $(n=1)$. Small bowel resection was combined with a partial gastrectomy in a single patient. Additional individual patient data are summarized in Supplementary Table S2. 
Chapter 6

Table 1. Patient demographics and surgical characteristics at baseline.

\begin{tabular}{|c|c|}
\hline Variable & Study cohort \\
\hline Patients, $\mathrm{n}^{*}$ & 12 \\
\hline Age, yrs & $67.7 \pm 14.6$ \\
\hline Male/female & $7 / 5$ \\
\hline \multicolumn{2}{|l|}{ Underlying etiology, $n$ (\%) } \\
\hline Mechanical occlusion & $3(25)$ \\
\hline Ischemia & $4(33)$ \\
\hline Peritonitis & $5(42)$ \\
\hline PN dependence, $\mathrm{n}(\%)$ & $10(83)$ \\
\hline Jejunal efflux (mL/24 hrs) & 2245 [1366 to 2913$]$ \\
\hline Jejunal efflux (mL•weight ${ }^{-1} \cdot 24$ hrs $\left.^{-1}\right)$ & $40[22$ to 55$]$ \\
\hline Hospital admission time before referral (days) & $28[18$ to 59$]$ \\
\hline Plasma citrulline $\leq 20 \mu \mathrm{mol} / \mathrm{L}, \mathrm{n}(\%)$ & $7(58)$ \\
\hline SB resection $\geq 30 \mathrm{~cm}, \mathrm{n}(\%)$ & $4(50)$ \\
\hline Resection SB length if resection $(n=6), \mathrm{cm}$ & $60[15$ to 113$]$ \\
\hline Upstream SB length $(n=9), \mathrm{cm}$ & 120 [88 to 210$]$ \\
\hline Upstream SB length < $100 \mathrm{~cm}, \mathrm{n}(\%)$ & $2[22]$ \\
\hline Downstream SB length, cm & $1670[1300$ to 222$]$ \\
\hline Theorical total SB length** $(n=98), \mathrm{cm}$ & 310 [223 290 to 350] \\
\hline \multicolumn{2}{|l|}{ Downstream SB anatomy, n (\%) } \\
\hline Ileo-colon-rectum & $8(67)$ \\
\hline Ileo-colon-stoma & $4(33)$ \\
\hline \multicolumn{2}{|l|}{ Serum liver tests (IU/L) } \\
\hline ALP & 216 [163 to 291$]$ \\
\hline GGT & 176 [73 to 252] \\
\hline ALT & 66 [45 to 109$]$ \\
\hline AST & 37 [29 to 57$]$ \\
\hline Total bilirubin, $\mu \mathrm{mol} / \mathrm{L}$ & 6.2 [3.7 to 9.3$]$ \\
\hline Serum CRP (mg/L) & $6.3[2.1$ to 36.0$]$ \\
\hline
\end{tabular}

* If data are missing, numbers in parentheses indicate the number of assessments for a particular factor. Data are depicted as percentages (\%), mean \pm SD or median [IQR]. Abbreviations: PN, parenteral nutrition; $\mathrm{SB}$, small bowel; ALP, alkaline phosphatase; GGT, y-glutamyl transferase; ALT, alanine aminotransferase; AST, aspartate aminotransferase. ${ }^{* *}$ Theoretical total SB length = upstream + downstream SB length

\section{Dysregulated bile salt-FGF19 axis in intestinal failure patients with a temporary double enterostomy}

First, we investigated whether the bile salt-FGF19 axis was disturbed in IF-TDE patients prior to CR. Baseline levels of TBS, FGF19 and C4 of healthy controls and IFpatients are shown in Figure 1A. Note, demographic information on healthy controls 
are provided in Supplementary Table S3. TBS levels in IF patients were not different compared to healthy controls (1.9 [1.4 to 3.0$]$ vs $1.6[0.9$ to 2.4$] \mu \mathrm{mol} / \mathrm{L}, P=.288)$. In line with earlier observations in patients with chronic $\mathrm{IF}^{14}$, baseline FGF19 levels were markedly lower compared to healthy controls (0.023 [0.012 to 0.061$]$ vs 0.098 [0.071 to 0.136$] \mathrm{ng} / \mathrm{mL}, P<.001$ ) (Figure 1A). Conversely, high baseline C4 levels were observed in IF patients compared to controls (95 [38 to 169] vs 19 [10 to 31] ng/mL, $P$ $=.002$ ), indicating dysregulated control of bile salt synthesis (Figure 1A). Indeed, FGF19 showed a strong negative correlation with C4 at baseline $(\rho=-0.86, P<.001)$ (Figure 1B). Furthermore, baseline C4 levels were positively related to $24 \mathrm{hrs}$ jejunal output adjusted by baseline weight $(\rho=-0.80, P=.002)$ (Figure 1C), indicating a choleretic effect before CR that is linked to enhanced bile salt synthesis ${ }^{25}$. FGF19 levels were not related to jejunal efflux $(\rho=-0.44, P=.155)$ (Figure 1C). Of note, baseline C4 levels were higher in females compared to males (179 [95 to 274] vs 55 [28 to 103] $\mathrm{ng} / \mathrm{mL}, \mathrm{P}=.017$ ), but $\mathrm{C} 4$ levels of both sex groups of patients were significantly higher compared to healthy controls (data not shown). No clinical or biochemical explanation was found for the sex difference. None of the other laboratory parameters were different between males and females.

A
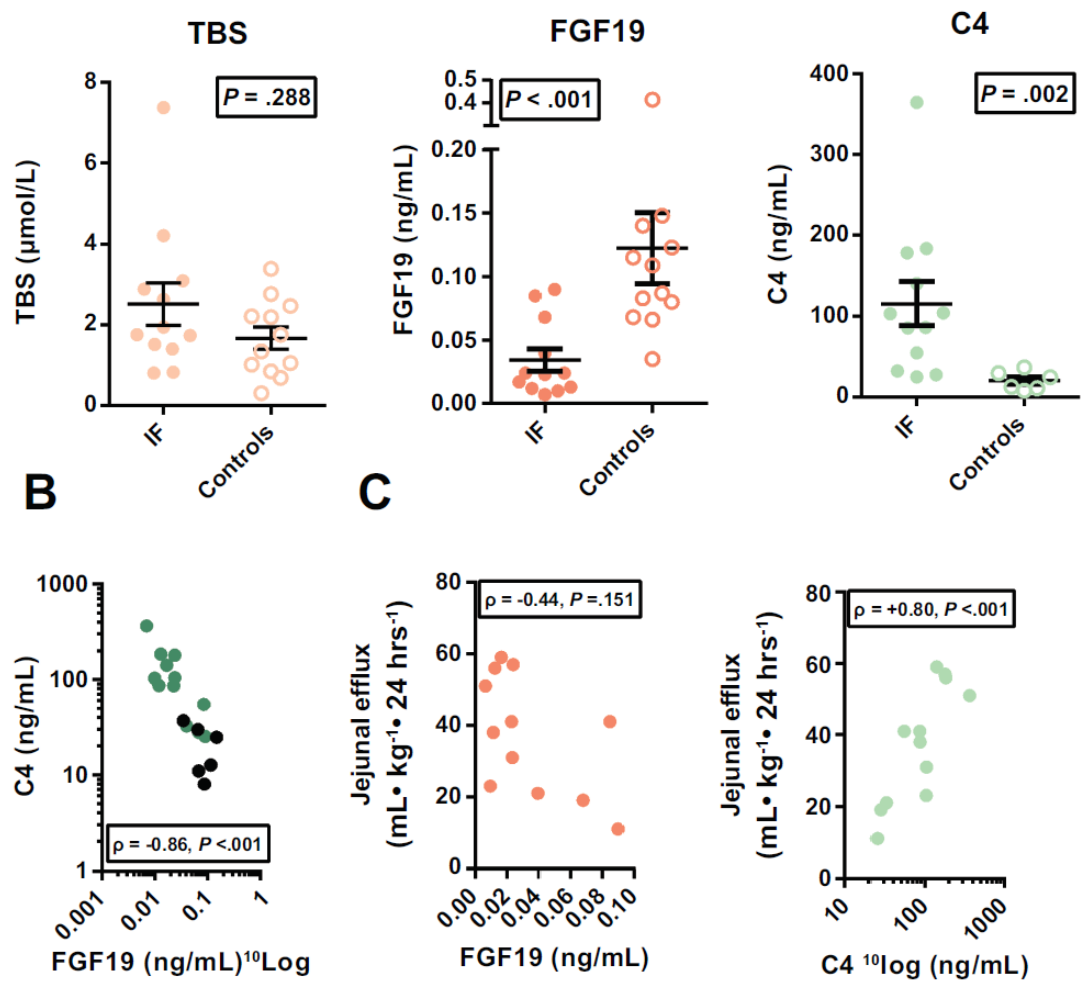

Figure 1. Dysregulated bile salt synthesis in IF patients with a temporary double enterostomy prior 
to CR. (A) Baseline values of TBS, FGF19 and C4 of IF patients with a TDE $(n=12)$ compared with controls $(n=12)$. Note, C4 levels were available for 6 out of 12 healthy volunteers. (B) Correlation between FGF19 and C4 levels in IF patients with a TDE (blue circles) and controls (black circles). (C) Correlation between jejunal efflux adjusted by weight, and FGF19 or C4. Data is depicted as mean $\pm 95 \% \mathrm{Cl}$. Differences were evaluated by Mann-Whitney $U$ test. Correlations were evaluated with Spearman's $(\rho)$ correlation coefficient. $P$ values are depicted.

\section{Chyme reinfusion restores the regulatory bile salt-FGF19 axis}

Reinfusion of proximal small intestinal secretions into the distal small bowel allows enterohepatic recycling of bile salts to the liver and may accordingly reinstate the gutliver axis of bile salt-FGF19 signaling. For this reason, we studied the effect of CR on the time courses of plasma TBS, FGF19 and C4. The individual responses are shown in Supplementary Figures $2 A$ \& 2B. Systemic TBS levels did not change significantly after introducing CR $(P=.112)$ (Figure 2A). FGF19 levels increased after CR initiation $(P=.002)$ with a transient peak after 7 days, and reaching a new steady-state level that was higher than at baseline, thereafter (Figure 2A). In line with elevated FGF19 levels, plasma C4 levels were reduced after start of $\operatorname{CR}(P<.001)$ (Figure 2A), indicating reduced bile salt synthesis during $C R$. The percentage change from baseline is shown in Figure 2B. Most pronounced responses of FGF19 and C4 were seen after 7 days of CR (Figure 2B). TBS levels were unaltered after 7 days of CR (1.9 [1.4 to 3.0] vs 1.2 [0.5 to 3.6] $\mu \mathrm{mol} / \mathrm{L}, P=.773$ ) (Figure 2C) FGF19 levels were higher for all but one patient after 7 days compared to baseline values $(0.023$ [0.012 to 0.061$]$ vs 0.128 [0.077 to 0.208] ng/mL, $P=.003$ ) (Figure 2C). Conversely, and in line with FGF19 function, C4 levels were consistently lower after 7 days compared to baseline values (95 [38 to 196] vs [18 [11 to 26$] \mathrm{ng} / \mathrm{mL}, P<.001)$ (Figure 2 C).

Further, gene expressions of FXR and the FXR-regulated organic solute transporter subunits $\alpha / \beta$ (OST $\alpha / \beta$, engaged in export of bile salts from the enterocyte to the venous capillaries) were upregulated after $\mathrm{CR}(P<.05$ for all) (Figure 2D). Transcripts of the apical sodium-dependent bile salt transporter $(A S B T)$, implicated in uptake of bile salts from the intestinal lumen were not altered after $C R(P=.612)$ (Figure 2D). Expression of DIET1, a modulator of FGF19 secretion ${ }^{26}$, was significantly increased after 21 days (Figure 2D). For reasons unknown, FGF19 mRNA was not detected in the majority of specimens, irrespective of sampling prior to, or after 3 weeks of CR (data not shown). 
Eleven patients (92\%) had jejunal effluxes above $1200 \mathrm{~g} / 24 \mathrm{hrs}$, and a single patient had an output of $678 \mathrm{~g} / 24 \mathrm{hrs}$ prior to $\mathrm{CR}$. Inherent to the design of the device for reinfusing chyme, jejunal efflux could not be quantified during the course of CR. All patients developed stools or colonic stoma output after $\mathrm{CR}$, with a single patient having persistently a high fecal output as a consequence of diarrhea. Nevertheless, intestinal output declined significantly during CR (2245 [1366 to 2913] vs 203 [125 to 375] g/24 hrs, $P<.001)$ (Figure 2E).

A
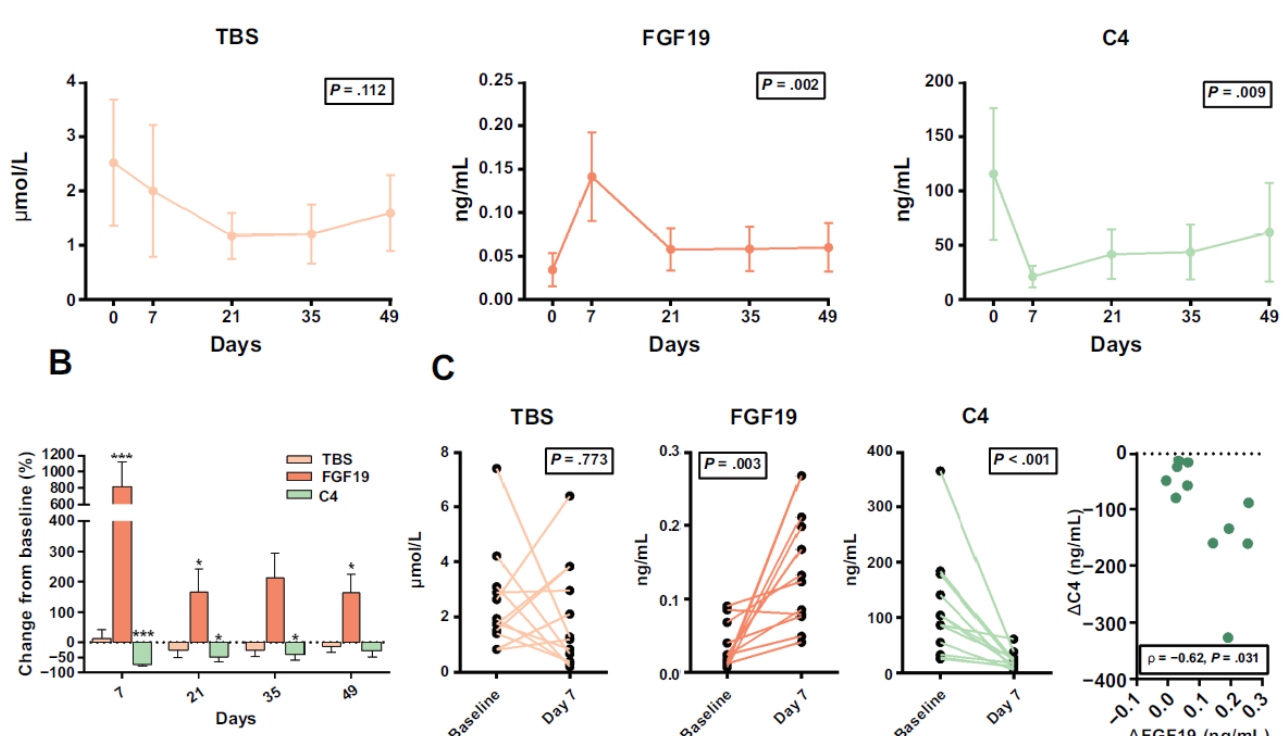

C

TBS
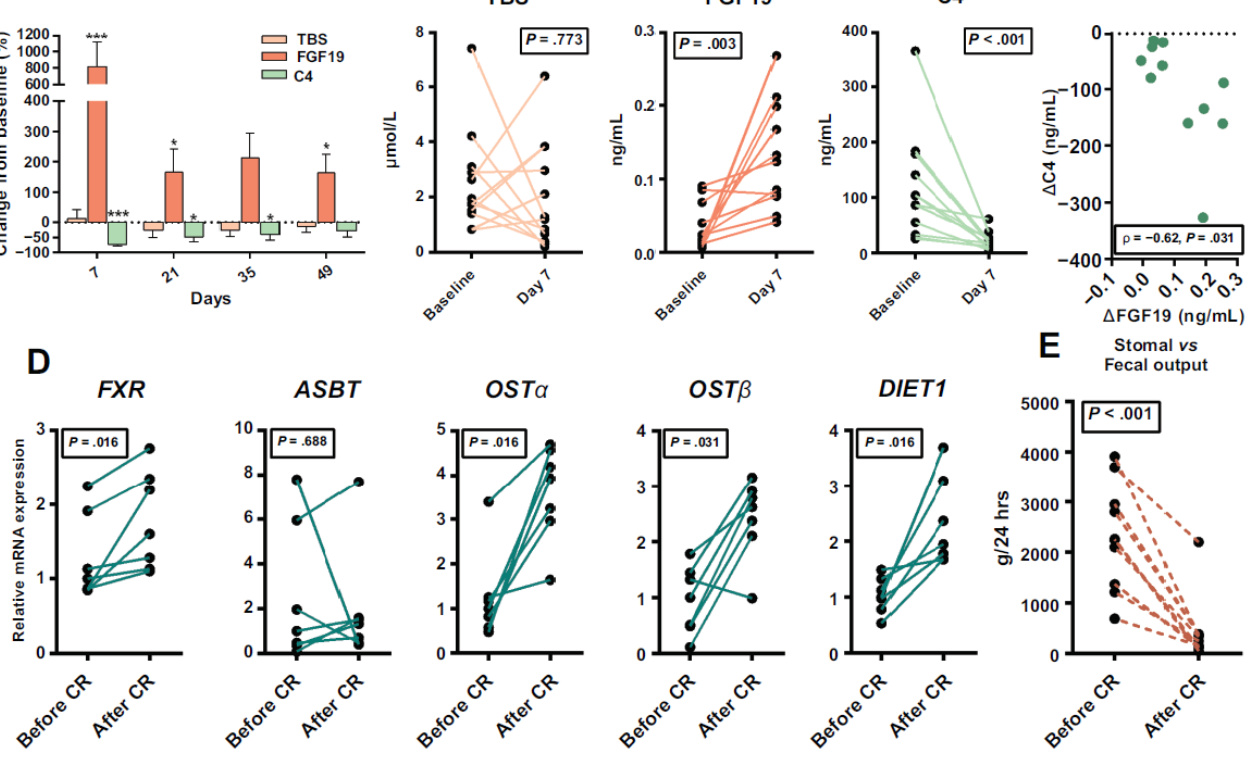

E $\begin{gathered}\text { Stomal vs } \\ \text { Fecal output }\end{gathered}$

Figure 2. CR restores homeostatic control of bile salt synthesis within 7 days. Twelve patients with a temporary double enterostomy on PN underwent CR for up to 7 weeks. Blood was sampled on the depicted time points before and after start of CR, and analyzed for (A) TBS, FGF19 and C4. (B) Relative change from baseline over time for TBS, FGF19 and C4. (C) Individual changes of TBS, FGF19 and C4 in the first week of CR. (D) Paired small intestinal biopsies were studied ( $n=7$ patients) at 3 days before $\mathrm{CR}$ and 21 days after $\mathrm{CR}$ and analyzed for mRNA expression of $F X R$ and genes related to bile salt transport. (E) Proximal jejunal output at start of $\mathrm{CR}$, and stomal or fecal output at discharge from the clinic/end of CR were assessed to address intestinal output changes. Data is depicted as mean $\pm 95 \% \mathrm{Cl}$. Trends in time were evaluated by ANOVA with repeated measures. mRNA expression and intestinal output 
differences were evaluated by Wilcoxon matched-pairs signed ranks sum test. $P$ values are depicted. Asterisks indicate significance levels: ${ }^{*}(P<.05),{ }^{* *}(P<.01)$ and ${ }^{* * *}(P<.001)$.

\section{Chyme reinfusion alters composition of circulating bile salts}

Re-entry of chyme into the distal small bowel and colon restores interaction between the gut microbiota and host bile salts and results in microbial bile salt metabolism ${ }^{27}$. At baseline, gut microbiota-derived secondary bile salts and deconjugated bile salt species were virtually absent (Figure $3 A \& 3 B$ ). The molar fractions of secondary and deconjugated bile salt species increased during CR treatment $(P=.004$ and $P<.001$, respectively) and reflect the restored contact between bile salts and bile saltmetabolizing gut microbes (Figure $3 A \& 3 B$ ). Moreover, the fractions of glycineconjugated bile salts were elevated after $\mathrm{CR}(P=.025)$ (Figure $3 C)$, also mirrored by lower ratio of taurine- to glycine-conjugated bile salts $(P=.008)$ (Supplementary Figure S3A). Individual plasma bile salt species are reported in Figure $3 E$. Note, absolute plasma levels of a number of secondary bile salts ((glyco)deoxycholate, (glyco)lithocholate and ursodeoxycholate) (all $P<.05)$ ) were significantly increased after 7 days of CR treatment (Figure 3E). An overview of absolute levels of all detected bile salt species at the respective time points, is provided in Supplementary Table S4. Absolute levels of the most abundant sulfated bile salt species (i.e. glycochenodeoxycholate-3-sulfate, GCDCA-3S), which are typically formed under conditions of cholestasis ${ }^{28}$, were reduced after CR $(P=.049)$ (Figure 3D), and showed a positive correlation with hepatobiliary injury markers ALP and GGT $(\rho=+0.65, P$ $=.026$ and $\rho=+0.50, P=.104$, respectively) (Supplementary Figure S3B). Finally, GCDCA, GCA and TCA were strongly correlated to ALT $(\rho=+0.66, P=.024, \rho=+0.83$, $P=.002$ and $\rho=+0.76, P=.006$, respectively) (Supplementary Figure S3C).

Furthermore, we analyzed individual chyme bile salt species at baseline and after 7 days of CR treatment. Mole fractions of individual bile salt species in chyme did not show major shifts (Figure 3F). Note, the high fraction of unconjugated bile salt species $(50.1 \%)$. Intriguingly, the total fraction of unconjugated bile salt species in chyme (i.e. CA, CDCA and DCA) was unexpectedly high, considering that $>99 \%$ of biliary bile salts are conjugated ${ }^{29}$. A possible explanation could be bacterial overgrowth in the proximal small intestine and attendant microbial bile salt deconjugation.

Re-introducing bile salts in the distal small bowel leads to FGF19 production. In support 
of this notion, mole fractions of GCA $(\rho=+0.71, P=.014)$ and GCDCA $(\rho=+0.71, P$ $=.014$ ) were strongly associated with plasma FGF19 levels (Figure 3G), but significant associations with plasma C4 levels were not found (Figure 3G).

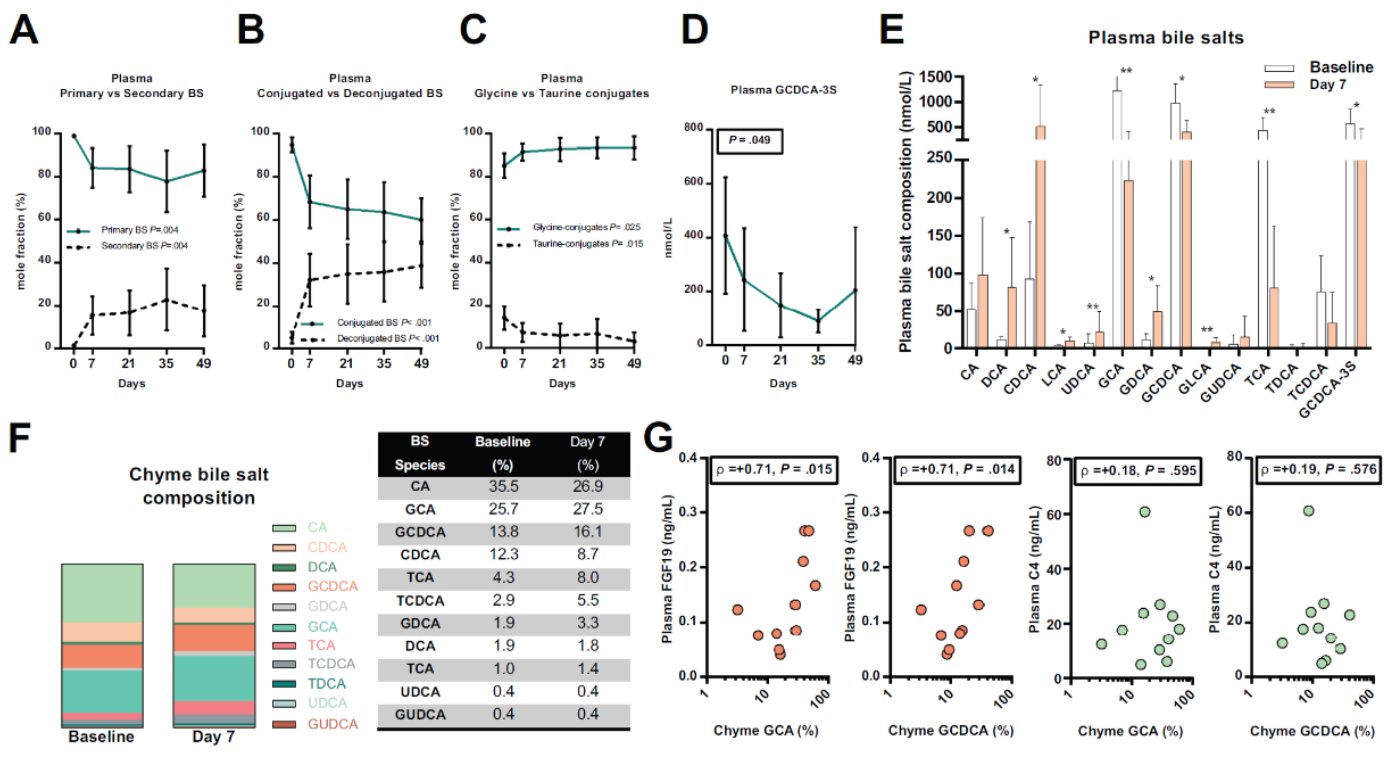

Figure 3. Alteration of serum bile salt composition after CR initiation. Time course of circulating bile salt composition during $C R(n=12)$. Graphs show time courses of $(\mathbf{A})$ primary and secondary bile salts, (B) deconjugated and conjugated bile salts, (C) glycine- and taurine-conjugated bile salts and (D) the sulfated bile salt species GCDCA-3S. (E) Individual plasma bile salt species. (F) Chyme bile salt composition, fraction of individual bile salt species (\%) of total bile salt composition. (G) Correlation between chyme GCA and GCDCA and plasma levels of FGF19 and C4. Data is depicted as mean $\pm 95 \%$ $\mathrm{Cl}$. Trends in time were evaluated by ANOVA with repeated measures. $P$ values are depicted. Asterisks indicate significance levels: ${ }^{*}(P<.05)$ and ${ }^{* *}(P<.01)$.

\section{Chyme reinfusion restores intestinal function and protects against liver injury}

CR restored endocrine regulation of bile salt synthesis and allowed microbial metabolism of bile salts. Next, we studied whether CR recovered intestinal function and ameliorated liver injury. We first assessed whether CR could improve intestinal function. At baseline, 8 patients had plasma CIT concentrations $\leq 20 \mu \mathrm{mol} / \mathrm{L}$ (Figure $\mathbf{4 A}$ ), indicating impaired intestinal function ${ }^{30}$. Remaining 4 patients (2 on PN) with higher CIT levels had a combination of persistent high jejunal efflux (median $2170 \mathrm{~mL}$ ) and long period of hospital admission (median 47 days) prior to $\mathrm{CR}$, justifying $\mathrm{CR}$ treatment. CIT concentrations went up after CR initiation $(P=.004)$ and levels considered normal ${ }^{30}$ were attained after 5 weeks in all patients (Figure $4 A$ ). Serum 
albumin levels increased after $\mathrm{CR}$ initiation $(P<.001)$ and were in the normal range for 6 of 12 patients (50\%) after 7 weeks (Figure $4 B$ ). Individual plasma CIT and albumin time courses are depicted in Supplementary Figure S4. Expression of the epithelium-specific genes villin-1 (VIL1, cytoskeletal component), sucrase-isomaltase ( $S$ I, carbohydrate digestive enzyme) and cubilin (CUBN, engaged in vitamin B12 absorption were determined to study the impact of $\mathrm{CR}$ on enterocyte function. CR significantly increased VIL1 $(P=.016)$ and $\mathrm{SI}(P=.031)$ mRNA levels, whereas CUBN expression levels were not altered during CR $(P=.156)$ (Figure 4D). Further, the mRNA level of ornithine carbamoyl transferase (OCT, involved in CIT synthesis) was increased after CR $(P=.016)$, in line with increased plasma CIT levels after 21 days of $C R$ (Figure $4 A \& 4 C$ ). We evaluated the local inflammatory response (small intestinal IL6 mRNA level). IL6 mRNA expression was decreased after CR $(P<.001)$. In addition, transcript levels of pro-inflammatory cytokines including $T N F \alpha$ and $I L-1 \beta$ (Figure 4D), IL-8 and IL-17 (Supplementary Figure S5) were decreased after three weeks of $C R$ treatment (all $P<.05)$. mRNA levels of the anti-inflammatory cytokine IL10 was also decreased after three weeks of CR treatment (Figure 4D).

Secondly, we studied the effect of CR on liver injury. Serum baseline elevations of ALP and GGT were significantly reduced after 49 days of CR $(P<.001$ and $P=.003$, respectively), approaching the respective normal ranges after 35 days (Figure $4 E$ ). Total bilirubin levels were within the normal range at baseline and were not affected by CR $(P=.408)$ (Figure $4 E)$. ALT and AST levels were above the normal range at baseline, although the time course displayed declining trends in time, these were not significant $(P=.150$ and $P=.189$, respectively) (Supplementary Figure S6).

Finally, we evaluated the systemic inflammatory response (CRP and pro-inflammatory cytokine levels). Serum CRP were at relatively low levels and decreased nonsignificantly during CR $(P=.052)$ (Figure 4F). We also tested systemic levels of proinflammatory cytokines IL6 and TNF- $\alpha$, but found levels to be below the detection limit of the immune assays for 10 and 12 out of 12 patients, respectively (data not shown), highlighting the non-inflammatory state of the patients before and during $\mathrm{CR}$.

\section{Improvement of the nutritional status by chyme reinfusion}

During $\mathrm{CR}$, the oral feeding met the recommended dietary allowance for energy and protein intake. Three patients with residual malabsorption received additional enteral nutrition by "en Y" enteroclysis. Consequently, PN and/or IVS could be stopped in 9 
out of 10 patients $(90 \%)$ within a median of 3 [1 to 9] days after start of CR. Most patients $(n=8)$ had poor nutritional status before $C R$, but this improved during CR (Supplementary Table S5).
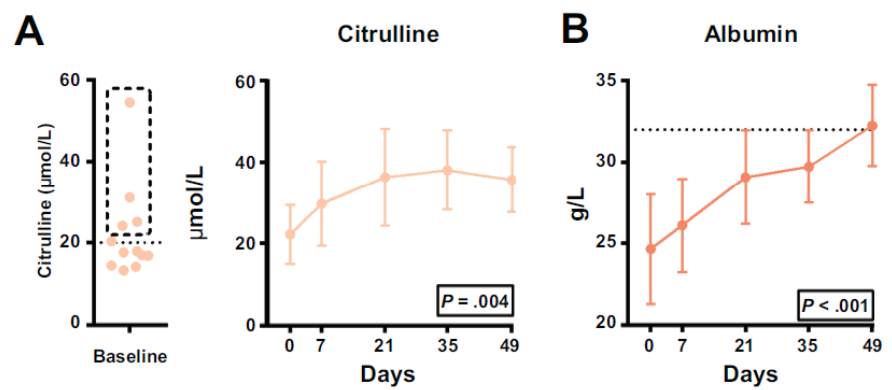

C

Intestinal epithelial-specific genes and local inflammation

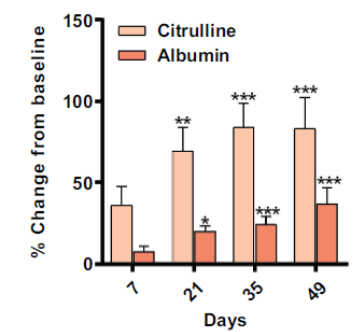

D
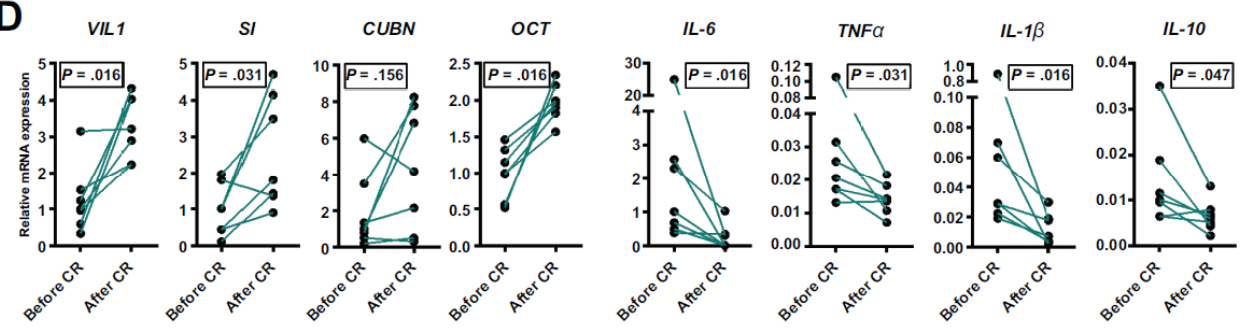

Liver injury and systemic inflammation

E

ALP

GGT

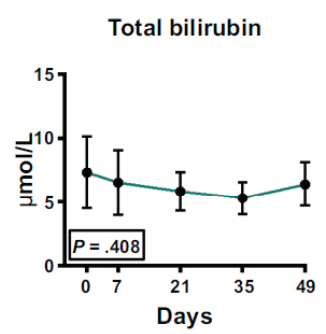

$\mathbf{F}$

CRP
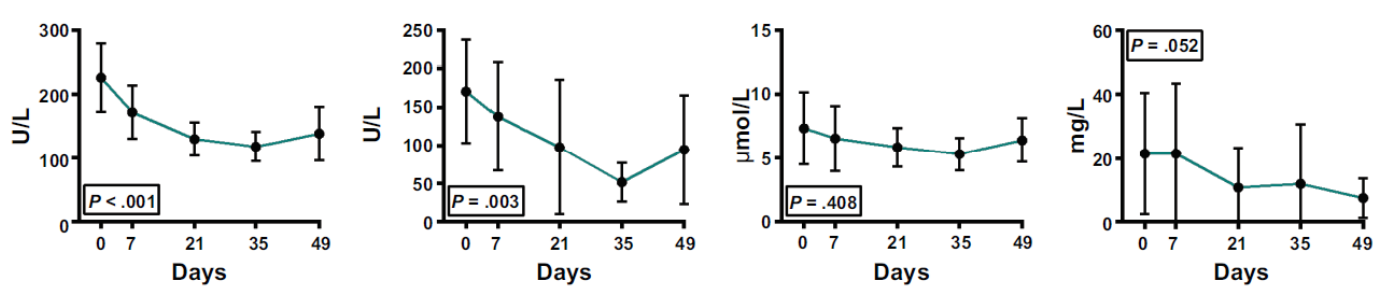

Figure 4. CR recovers intestinal absorptive function and ameliorates liver injury. Time course of markers for intestinal absorptive function and nutritional status during CR. (A) CIT values of individual patients at baseline (left panel) with values $>20 \mu \mathrm{mol} / \mathrm{L}$ in a dashed box and time course of CIT (right panel). (B) Time course of albumin (dashed lines represents normal value). (C) Relative change from baseline over time for CIT and albumin. (D) Paired mRNA expression of epithelium specific genes (VIL-1,

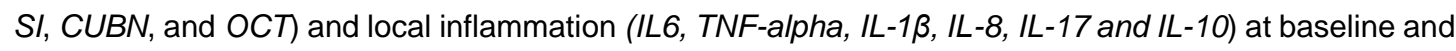
3 weeks after CR. $€$ Time course of liver injury markers (ALP, GGT and total bilirubin) and (F) systemic inflammation (CRP). Data is depicted as mean $\pm 95 \% \mathrm{Cl}$. Trends in time were evaluated by ANOVA with repeated measures. Gene expression differences were evaluated by Wilcoxon matched-pairs signed ranks sum test. $P$ values are depicted.

The C4 response after 7 days dependent of the FGF19 response highly predicts 


\section{the course of CIT}

Evaluation of plasma time courses of FGF19 and C4 showed that the reciprocal response in the first week was followed by a gradual increase of CIT levels and decrease of liver injury markers (ALP and GGT) (Figure 1 and Figure 4). Therefore, we constructed a linear mixed model with random intercepts to evaluate the association between the change in FGF19 and C4 after 7 days of CR $(\Delta)$ and alterations in markers of intestinal function (CIT) and liver injury (ALP and GGT) from day 7 onwards. First, the reciprocal interaction between $\Delta_{\mathrm{FG} 19}{ }^{*} \Delta_{\mathrm{C} 4}$ significantly predicted CIT values over time (estimate: $-.54,95 \% \mathrm{Cl}$ : [-.91 to -.18], $P=.007$ ). The interaction plot $\left(\mathrm{R}^{2}=+0.67, P=.007\right)$ is depicted in Figure $\mathbf{5}$ and demonstrates that the FGF19 response within the first 7 days, dependent on the $C 4$ response, predicts CIT values over time. Finally, the $\Delta_{\mathrm{FGF} 19}$ (per 0.1 unit increase) predicts the course of ALP (estimate: $-22.0,95 \% \mathrm{Cl}$ : [-40.2 to -3.8$], P=.022$ ) and tended to predict GGT (estimate: $-26.8,95 \% \mathrm{Cl}$ : [-58.3 to 47.6$], P=.095)$ (data not shown).

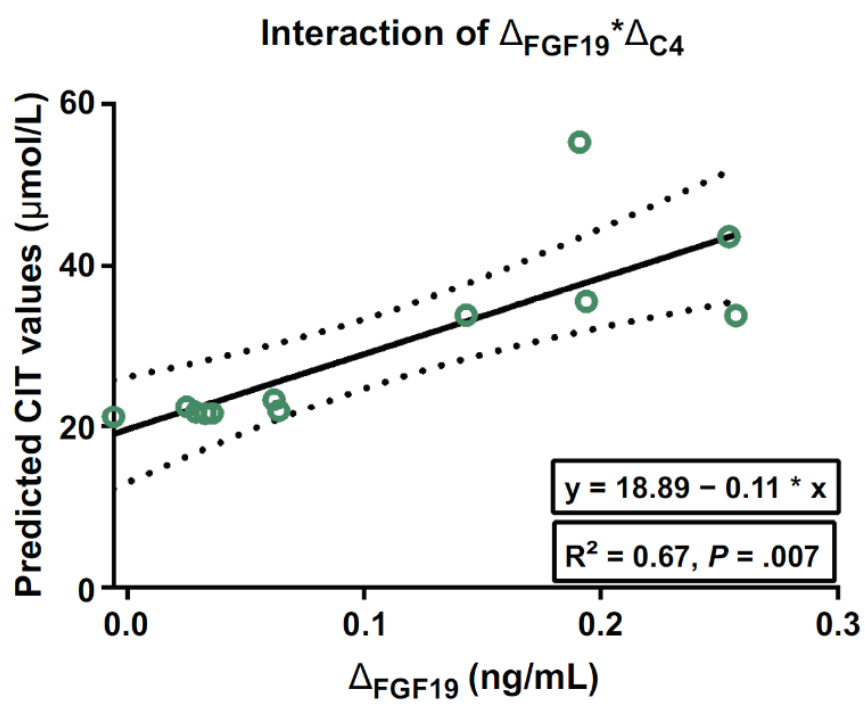

Figure 5. The $\mathrm{C} 4$ response, dependent on the FGF19 response, after 7 days predicts the plasma course of CIT over time. A linear mixed effects model was constructed to evaluate whether the response of FGF19 and C4 (difference between 7 days and baseline values $=\Delta$ ) predicts the course of CIT over

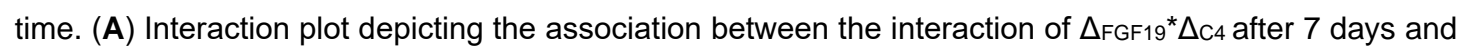
predicted CIT values over time (from 7 days onwards). The $\Delta_{\text {FGF19 }}(\mathrm{ng} / \mathrm{mL}$ ) is depicted on the $x$-axis and the $\Delta_{\mathrm{C} 4}(\mathrm{ng} / \mathrm{mL})$ is shown at the corresponding dots. The $y$-axis shows the predicted CIT values $(\mu \mathrm{mol} / \mathrm{L})$. The regression line is shown with $95 \%$ confidence interval. R-squared and $P$ values are depicted. 


\section{Discussion}

CR re-establishes small intestinal continuity and hence functionally restores the enterohepatic circulation of bile salts. In this prospective study, the key finding is that CR rapidly reinstates the regulatory bile salt-FGF19 axis and further restores intestinal function, reverses liver injury and contributes to the improvement of the nutritional status in IF patients with a TDE. Our findings support a role for recovery of homeostatic control of bile salt synthesis by CR in patients with IF with a TDE. The pathophysiology and mechanistic findings were schematically summarized in Figure 6 .

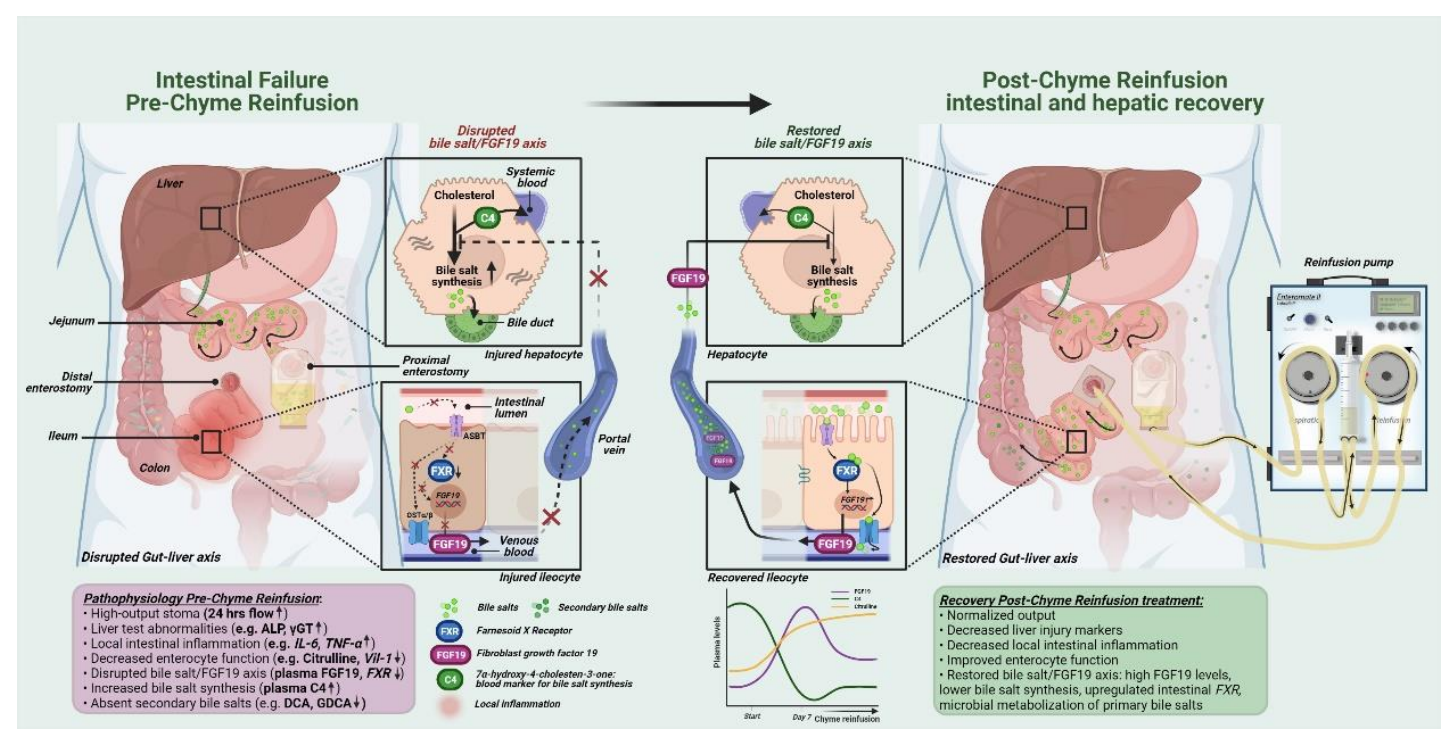

Figure 6. The pathophysiology and mechanistic findings in the present study.

To our knowledge the bile salt-FGF19 axis was not previously investigated in IF patients undergoing extracorporeal recovery of intestinal continuity. In our study, we observed that endocrine control of bile salt synthesis was recovered after 7 days of $C R$ followed by a new equilibrium phase with higher FGF19 levels compared to the preCR period. This is in line with studies showing that administration of an FGF19 analogue strongly suppressed serum C4 levels in healthy human volunteers within 7 days and in patients with chronic liver disease after 4 or 12-weeks of treatment ${ }^{31-33}$. The restored regulatory bile salt-FGF19 axis, evidenced by decline in C4 levels, was an anticipated effect of reinstating the enterohepatic circulation of bile salts. Furthermore, functional recovery of the enterohepatic circulation of bile salts was also reflected by larger abundance of microbiome-derived secondary and deconjugated bile salt species, indicating restored microbial metabolism of the gut ${ }^{16}$.

The initial response of FGF19 and C4 levels after 7 days of CR was followed by 
gradually increasing CIT levels, indicating that restoration of the bile salt-FGF19 axis preceded recovery of intestinal function. Interestingly, the initial FGF19 response, dependent on C4, was highly associated with the later course of CIT over time. This would imply that restoring endocrine control of bile salt synthesis is an early step in functional recovery of the intestine. A recent study demonstrated involvement of FGF19 in preserving intestinal barrier integrity and protection against intestinal inflammation mediated by $\mathrm{FXR}^{34}$.

Our findings also demonstrated upregulated mRNA expression of the bile salt sensor FXR, the bile salt transporters $O S T \alpha / \beta$, and epithelium specific genes $V I L-1, S I$ and OCT in the distal small intestine after 21 days of CR. Interestingly, FXR expression was negatively related to the pro-inflammatory cytokine (IL6) and positively related to intestinal epithelial-specific genes (VIL1, SI, CUBN and OCT) (data not shown). Mechanistically, this may be attributed to normalized trans-epithelial bile salt flux facilitated by $C R$, hence intestinal FXR activation. Bile salts play a direct role in preserving the intestinal barrier function and protection against intestinal inflammation, effects mediated by FXR ${ }^{35}$.

Remarkably, findings in the present study showed that jejunal output prior to CR was strongly related to systemic $\mathrm{C} 4$ levels. A plausible explanation could be that enhanced synthesis and biliary secretion of bile salts induces a choleretic effect and eventually elevated jejunal output ${ }^{36}$. Since the enterohepatic circulation of bile salts was recovered and $\mathrm{C} 4$ levels were normalized after $\mathrm{CR}$, it is likely that reduced stomal or fecal output after CR treatment was, in part, due to restoration of the ileal brake ${ }^{6}$, a direct consequence of recovery of the bile salt-FGF19 axis. Indeed, patients with chronic primary bile acid diarrhea have high $\mathrm{C} 4$ levels ${ }^{37}$. Note, a single patient remained to have a high fecal output (>2 liters per day) after 49 days of CR treatment. This patient had persistently the lowest FGF19 and highest C4 level during the course of $\mathrm{CR}$. Intriguingly, this patient underwent a partial gastrectomy prior CR. High gastrin levels are observed after gastrectomy and are related to increased choleresis ${ }^{38,39}$.

The major strength of our study was long term follow-up with multiple sampling points, which allowed us to analyze the time course of homeostatic control of bile salt synthesis. This study has certain limitations. First, considering the complexity of IF, we designed a pre- and post-intervention study without any control group without CR treatment. Importantly, CR is recommended, whenever feasible, by ASPEN guidelines 
and ESPEN recommendations. Withholding this effective treatment for the purpose of a controlled study, would have been unethical. In addition, patients already had a median pre-CR period of 4 weeks with unsuccessful treatment with PN or IVS. Secondly, ileal biopsies were taken 3 days before and 3 weeks after CR. However, changes in FGF19 and C4 levels were rapidly seen after 7 days of CR treatment. Thus, sequential biopsies taken at each corresponding time point of blood collection would be more informative about transcriptional changes during $C R$. Obviously, frequent biopsies is a huge burden for patients and not justified by clinical necessity. Nonetheless, alterations in circulating FGF19 and C4 levels correspond well to transcriptional changes ${ }^{40,41}$.

The findings of this study make it conceivable that the beneficial effects of $C R$ are mediated in part through the restored bile salt-FGF19 signaling axis. Clinically, CR in surgical patients with a distal entrance of the small bowel should be considered in case IF develops, because CR might also benefit postoperative outcomes considering the persevering effects on intestinal function. In addition, in case patients need to undergo a temporary proximal enterostomy (e.g. during acute mesenteric ischemia), establishment of a double-barreled enterostomy or placement of a distal entrance of the small bowel should be considered. Finally, an important clinical implication of our study is that administration of FGF19 analogues could be considered in IF patients without an accessible distal small bowel.

In conclusion, CR improves intestinal and liver function in IF patients with a TDE. The beneficial effects of CR are partly mediated by activation of bile salt-FGF19 signaling leading to restored regulation of hepatic bile salt synthesis, and is associated with improved gut-liver health. CR needs to be considered as first-line treatment in IF patients with a TDE. Further, novel therapeutic approaches with FGF19 analogues might be promising in the management of IF and related complications in case a distal intestine is not accessible. 


\section{References}

1. Pironi L, Arends J, Baxter J, Bozzetti F, Pelaez RB, Cuerda C, et al. ESPEN endorsed recommendations. Definition and classification of intestinal failure in adults. Clin Nutr. 2015;34:171-80.

2. Oterdoom LH, Ten Dam SM, de Groot SD, Arjaans W, van Bodegraven AA. Limited long-term survival after in-hospital intestinal failure requiring total parenteral nutrition. Am J Clin Nutr. 2014;100:1102-7.

3. Burden S, Hemstock M, Taylor M, Teubner A, Roskell N, MacCulloch A, et al. The impact of home parenteral nutrition on the burden of disease including morbidity, mortality and rate of hospitalisations. Clin Nutr ESPEN. 2018;28:222-7.

4. Rinsema W, Gouma DJ, von Meyenfeldt MF, Soeters PB. Reinfusion of secretions from highoutput proximal stomas or fistulas. Surg Gynecol Obstet. 1988;167:372-6.

5. Levy E, Palmer DL, Frileux P, Parc R, Huguet C, Loygue J. Inhibition of upper gastrointestinal secretions by reinfusion of succus entericus into the distal small bowel. A clinical study of 30 patients with peritonitis and temporary enterostomy. Ann Surg. 1983;198:596-600.

6. Picot D, Garin L, Trivin F, Kossovsky MP, Darmaun D, Thibault R. Plasma citrulline is a marker of absorptive small bowel length in patients with transient enterostomy and acute intestinal failure. Clin Nutr. 2010;29:235-42.

7. Picot D, Layec S, Seynhaeve E, Dussaulx L, Trivin F, Carsin-Mahe M. Chyme Reinfusion in Intestinal Failure Related to Temporary Double Enterostomies and Enteroatmospheric Fistulas. Nutrients. 2020;12.

8. Inagaki T, Choi M, Moschetta A, Peng L, Cummins C, McDonald J, et al. Fibroblast growth factor 15 functions as an enterohepatic signal to regulate bile acid homeostasis. Cell metabolism. 2005;2:217-25

9. Cai SY, Ouyang X, Chen Y, Soroka CJ, Wang J, Mennone A, et al. Bile acids initiate cholestatic liver injury by triggering a hepatocyte-specific inflammatory response. $\mathrm{JCl}$ Insight. 2017;2:e90780.

10. Pereira-Fantini PM, Lapthorne S, Joyce SA, Dellios NL, Wilson G, Fouhy F, et al. Altered FXR signalling is associated with bile acid dysmetabolism in short bowel syndrome-associated liver disease. J Hepatol. 2014;61:1115-25.

11. Xiao YT, Cao Y, Zhou KJ, Lu LN, Cai W. Altered systemic bile acid homeostasis contributes to liver disease in pediatric patients with intestinal failure. Sci Rep. 2016;6:39264.

12. Koelfat KVK, Visschers RGJ, Hodin C, de Waart DR, van Gemert WG, Cleutjens JPM, et al. FXR agonism protects against liver injury in a rat model of intestinal failure-associated liver disease. J Clin TransI Res. 2018;3:318-27.

13. Mutanen A, Lohi J, Heikkila P, Jalanko H, Pakarinen MP. Loss of ileum decreases serum fibroblast growth factor 19 in relation to liver inflammation and fibrosis in pediatric onset intestinal failure. J Hepatol. 2015;62:1391-7.

14. Koelfat KVK, Huijbers A, Schaap FG, van Kuijk SMJ, Lenicek M, Soeters MR, et al. Low circulating concentrations of citrulline and FGF19 predict chronic cholestasis and poor survival in adult patients with chronic intestinal failure: development of a Model for End-Stage Intestinal Failure (MESIF risk score). Am J Clin Nutr. 2019;109:1620-9.

15. Walters JR, Tasleem AM, Omer OS, Brydon WG, Dew T, le Roux CW. A new mechanism for bile acid diarrhea: defective feedback inhibition of bile acid biosynthesis. Clin Gastroenterol Hepatol. 
2009;7:1189-94.

16. Hirschfield GM, Chazouilleres O, Drenth JP, Thorburn D, Harrison SA, Landis CS, et al. Effect of NGM282, an FGF19 analogue, in primary sclerosing cholangitis: A multicenter, randomized, double-blind, placebo-controlled phase II trial. J Hepatol. 2019;70:483-93.

17. Harrison SA, Rinella ME, Abdelmalek MF, Trotter JF, Paredes AH, Arnold HL, et al. NGM282 for treatment of non-alcoholic steatohepatitis: a multicentre, randomised, double-blind, placebocontrolled, phase 2 trial. Lancet. 2018;391:1174-85.

18. Picot D, Layec S, Dussaulx L, Trivin F, Thibault R. Chyme reinfusion in patients with intestinal failure due to temporary double enterostomy: A 15-year prospective cohort in a referral centre. Clin Nutr. 2017;36:593-600.

19. Aziz EF, Javed F, Pratap B, Musat D, Nader A, Pulimi S, et al. Malnutrition as assessed by nutritional risk index is associated with worse outcome in patients admitted with acute decompensated heart failure: an ACAP-HF data analysis. Heart Int. 2011;6:e2.

20. Schaap FG, van der Gaag NA, Gouma DJ, Jansen PL. High expression of the bile salthomeostatic hormone fibroblast growth factor 19 in the liver of patients with extrahepatic cholestasis. Hepatology. 2009;49:1228-35.

21. van Eijk HM, Rooyakkers DR, Deutz NE. Rapid routine determination of amino acids in plasma by high-performance liquid chromatography with a 2-3 microns Spherisorb ODS II column. J Chromatogr. 1993;620:143-8.

22. Axelson M, Aly A, Sjovall J. Levels of 7 alpha-hydroxy-4-cholesten-3-one in plasma reflect rates of bile acid synthesis in man. FEBS Lett. 1988;239:324-8.

23. Koelfat KVK, Plummer MP, Schaap FG, Lenicek M, Jansen PLM, Deane AM, et al. Gallbladder Dyskinesia Is Associated With an Impaired Postprandial Fibroblast Growth Factor 19 Response in Critically III Patients. Hepatology. 2019;70:308-18.

24. Ruijter JM, Ramakers C, Hoogaars WM, Karlen Y, Bakker O, van den Hoff MJ, et al. Amplification efficiency: linking baseline and bias in the analysis of quantitative PCR data. Nucleic Acids Res. 2009;37:e45.

25. Eusufzai S, Axelson M, Angelin B, Einarsson K. Serum 7 alpha-hydroxy-4-cholesten-3-one concentrations in the evaluation of bile acid malabsorption in patients with diarrhoea: correlation to SeHCAT test. Gut. 1993;34:698-701.

26. Vergnes L, Lee JM, Chin RG, Auwerx J, Reue K. Diet1 functions in the FGF15/19 enterohepatic signaling axis to modulate bile acid and lipid levels. Cell Metab. 2013;17:916-28.

27. Sayin S, Wahlström A, Felin J, Jäntti S, Marschall H-U, Bamberg K, et al. Gut microbiota regulates bile acid metabolism by reducing the levels of tauro-beta-muricholic acid, a naturally occurring FXR antagonist. Cell metabolism. 2013;17:225-35.

28. Masubuchi N, Sugihara M, Sugita T, Amano K, Nakano M, Matsuura T. Oxidative stress markers, secondary bile acids and sulfated bile acids classify the clinical liver injury type: Promising diagnostic biomarkers for cholestasis. Chem Biol Interact. 2016;255:83-91.

29. Hofmann A. The continuing importance of bile acids in liver and intestinal disease. Archives of internal medicine 1999.

30. Crenn P, Coudray-Lucas C, Thuillier F, Cynober L, Messing B. Postabsorptive plasma citrulline concentration is a marker of absorptive enterocyte mass and intestinal failure in humans. Gastroenterology. 2000;119:1496-505.

31. Hirschfield GM, Chazouilleres O, Drenth JP, Thorburn D, Harrison SA, Landis CS, et al. Effect of 


\section{Chapter 6}

NGM282, an FGF19 analogue, in primary sclerosing cholangitis: A multicenter, randomized, double-blind, placebo-controlled phase II trial. J Hepatol. 2018.

32. Luo J, Ko B, Elliott M, Zhou M, Lindhout DA, Phung V, et al. A nontumorigenic variant of FGF19 treats cholestatic liver diseases. Sci Transl Med. 2014;6:247ra100.

33. Mayo MJ, Wigg AJ, Leggett BA, Arnold H, Thompson AJ, Weltman M, et al. NGM282 for Treatment of Patients With Primary Biliary Cholangitis: A Multicenter, Randomized, Double-Blind, Placebo-Controlled Trial. Hepatol Commun. 2018;2:1037-50.

34. Gadaleta RM, Garcia-Irigoyen O, Cariello M, Scialpi N, Peres C, Vetrano S, et al. Fibroblast Growth Factor 19 modulates intestinal microbiota and inflammation in presence of Farnesoid $\mathrm{X}$ Receptor. EBioMedicine. 2020;54:102719.

35. Gadaleta RM, van Erpecum KJ, Oldenburg B, Willemsen EC, Renooij W, Murzilli S, et al. Farnesoid $X$ receptor activation inhibits inflammation and preserves the intestinal barrier in inflammatory bowel disease. Gut. 2011;60:463-72.

36. Erlinger S, Dhumeaux D. Mechanisms and control of secretion of bile water and electrolytes. Gastroenterology. 1974;66:281-304.

37. Walters JR, Johnston IM, Nolan JD, Vassie C, Pruzanski ME, Shapiro DA. The response of patients with bile acid diarrhoea to the farnesoid $X$ receptor agonist obeticholic acid. Aliment Pharmacol Ther. 2015;41:54-64.

38. Wilson SD, Schulte WJ, Meade RC. Longevity studies following total gastrectomy. In children with the Zollinger-Ellison syndrome. Arch Surg. 1971;103:108-15.

39. Zaterka S, Grossman MI. The effect of gastrin and histamine on secretion of bile. Gastroenterology. 1966;50:500-5.

40. Zhang JH, Nolan JD, Kennie SL, et al. Potent stimulation of fibroblast growth factor 19 expression in the human ileum by bile acids. Am J Physiol Gastrointest Liver Physiol 2013;304:G940-8.

41. Galman C, Arvidsson I, Angelin B, et al. Monitoring hepatic cholesterol 7alpha-hydroxylase activity by assay of the stable bile acid intermediate 7alpha-hydroxy-4-cholesten-3-one in peripheral blood. J Lipid Res 2003;44:859-66. 
Supplementary Table S1. Primer sequences.

\begin{tabular}{|c|c|c|}
\hline Gene & Forward sequence & Reverse sequence \\
\hline $36 B 4 t$ & CCTCGTGGAAGTGACATCGT & ATCTGCTTGGAGCCCACATT \\
\hline PSMB4† & CTCGGCCAGATGGTGATTGA & AGGCTACACCAAGCATGTCC \\
\hline REEP5† & ACTACATGCTGAAGTGTGGCT & TCCAGTCTGGTTTAGGTGCTC \\
\hline HPRT1† & CCTGGCGTCGTGATTAGTGAT & GGGCTACAATGTGATGGCCT \\
\hline FXRiso & ACAGAACAAGTGGCAGGTC & CTGAAGAAACCTTTACACCCCTC \\
\hline SLC10A2* & TCGACTCTGGGAGCATCGTA & CCGCGATGGACCCAATTTTA \\
\hline$S L C 51 A^{*}$ & ACCCTTTGCCCCATCAAGAG & GCACACGGCATAAAACGAGG \\
\hline SLC51B* & GGGTCTAAGGACCTCGTTGC & TTCTTTTCTGCTTGCCTGGATG \\
\hline DIET1 & AAACTGCACAACTGTGGGAGA & GCAGCTTCCTGTTGTGCTTG \\
\hline VIL1 & ATGAGCCATGCGCTGAACTT & GCATGGATGTGGCATCGAAC \\
\hline$S I$ & AGACACCTGCTGTTGATGAAAT & GAGAGTCATTCCACGGCCTC \\
\hline CUBN & GCGTCCTTTGTTAGCCGATG & GGGTGGACATGACGCTGTAA \\
\hline OTC & CACCTTCAGGCAGCTACTCC & GCAGCAACTTTAGCAGTCTTCA \\
\hline IL6 & CATCCTCGACGGCATCTCAG & TCACCAGGCAAGTCTCCTCA \\
\hline CXCL8* & GCTCTGTGTGAAGGTGCAGTT & ACCCAGTTTTCCTTGGGGTC \\
\hline IL1 $\beta$ & GCTCTGTGTGAAGGTGCAGTT & ACCCAGTTTTCCTTGGGGTC \\
\hline$T N F \alpha$ & TCCCCAGGGACCTCTCTCTA & GAGGGTTTGCTACAACATGGG \\
\hline IL17 & AАCCGATCCACСТСАССТTG & TCTCTTGCTGGATGGGGACA \\
\hline IL10 & ACATCAAGGCGCATGTGAAC & TAGAGTCGCCACCCTGATGT \\
\hline
\end{tabular}

tHousekeeping genes. *Synonyms: SLC10A2, ASBT; SLC51A, OSTa; SLC51B, OSTB; CXCL8, IL8. 


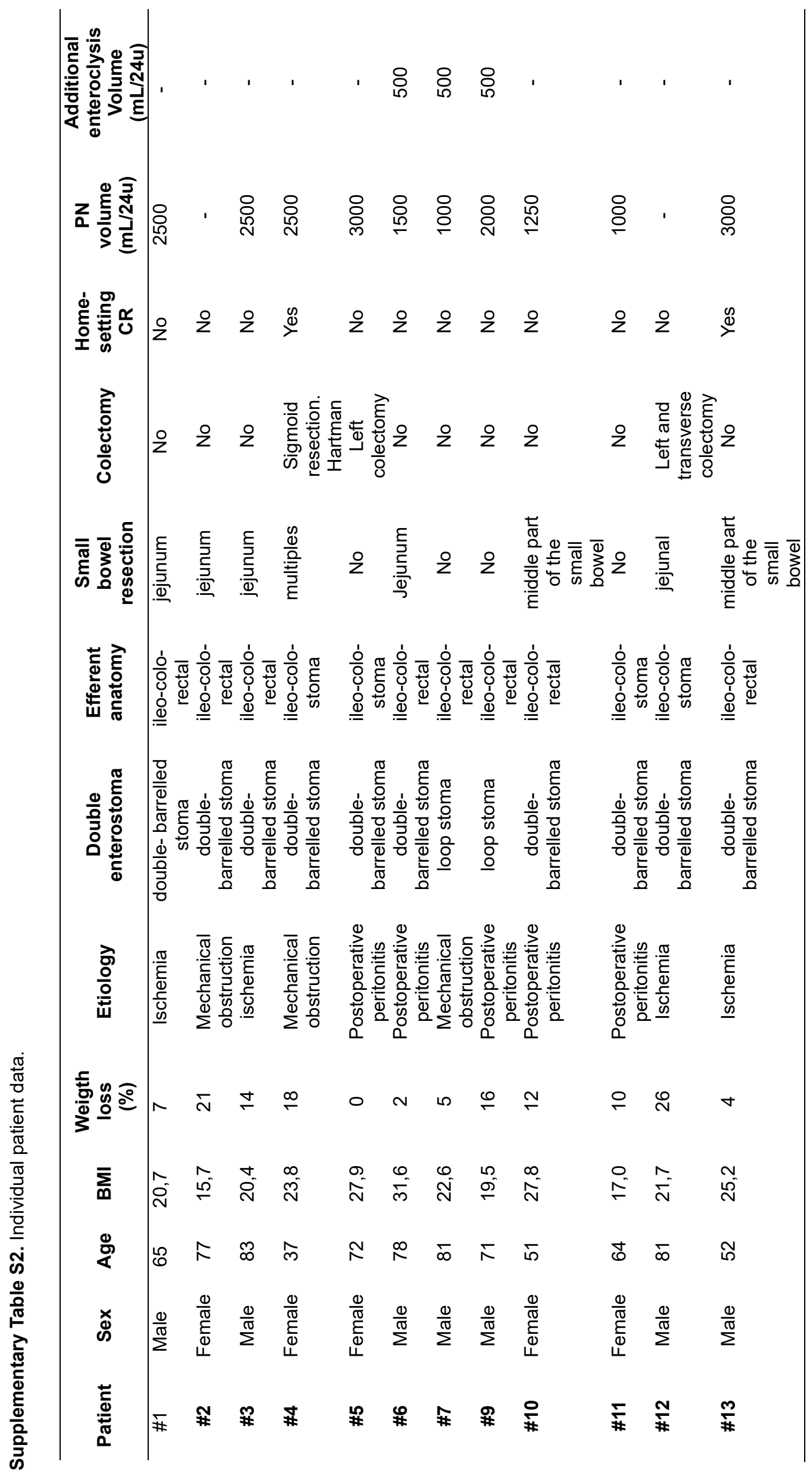


Supplementary Table S3. Demographics of healthy controls versus intestinal failure patients.

\begin{tabular}{lccc}
\hline Variable $(\mathbf{n}=\mathbf{1 2})$ & Healthy controls & IF-patients & $\boldsymbol{P}$ \\
\hline Age (years) & $54.7 \pm 19.7$ & $67.7 \pm 14.5$ & .079 \\
Sex (male vs female) & 8 vs 4 & 7 vs 5 & .178 \\
BMl (kg/m $)$ & $24.1 \pm 3.6$ & $22.8 \pm 4.7$ & .455 \\
\hline
\end{tabular}

Data are expressed as mean \pm standard deviation (SD). $P$ values in bold represent significant statistical differences. BMI indicates body mass index; Data of healthy controls are adapted from Plummer M.P., Crit Care Med. 2016 Sep;44(9):e790-6.

Supplementary Table S4. Overview of plasma bile salt species before and during chyme reinfusion.

\begin{tabular}{|c|c|c|c|c|c|c|c|c|c|c|c|c|c|c|c|}
\hline \multirow{2}{*}{$\begin{array}{l}\text { Bile salt } \\
\text { species } \\
\text { (nmol/L) }\end{array}$} & \multicolumn{3}{|c|}{ Baseline } & \multicolumn{3}{|c|}{7 days } & \multicolumn{3}{|c|}{21 days } & \multicolumn{3}{|c|}{35 days } & \multicolumn{3}{|c|}{49 days } \\
\hline & 31 & 13 & 79 & 83 & 18 & 110 & 70 & 24 & 106 & 44 & 27 & 114 & 76 & 38 & 152 \\
\hline$D C A$ & 12 & 6 & 13 & 57 & 6 & 94 & 29 & 8 & 92 & 48 & 6 & 130 & 35 & 12 & 109 \\
\hline$C D C A$ & 46 & 9 & 180 & 109 & 29 & 305 & 138 & 44 & 219 & 114 & 50 & 237 & 160 & 105 & 424 \\
\hline$L C A$ & 4 & 3 & 5 & 7 & 4 & 17 & 8 & 4 & 17 & 10 & 3 & 20 & 6 & 4 & 20 \\
\hline$U D C A$ & 0 & 0 & 2 & 6 & 2 & 19 & 6 & 1 & 61 & 6 & 3 & 17 & 7 & 3 & 8 \\
\hline$G C A$ & 892 & 716 & 1625 & 86 & 23 & 363 & 74 & 33 & 242 & 34 & 8 & 417 & 84 & 23 & 427 \\
\hline$G D C A$ & 9 & 5 & 10 & 30 & 5 & 77 & 14 & 5 & 65 & 25 & 7 & 50 & 21 & 7 & 40 \\
\hline$G C D C A$ & 885 & 404 & 1446 & 240 & 161 & 829 & 245 & 208 & 401 & 250 & 142 & 527 & 302 & 111 & 935 \\
\hline$G L C A$ & 0 & 0 & 0 & 5 & 0 & 14 & 0 & 0 & 10 & 5 & 0 & 29 & 3 & 0 & 11 \\
\hline GUDCA & 0 & 0 & 0 & 0 & 0 & 8 & 0 & 0 & 34 & 0 & 0 & 0 & 0 & 0 & 0 \\
\hline$T C A$ & 298 & 195 & 556 & 23 & 0 & 174 & 5 & 0 & 81 & 8 & 0 & 74 & 7 & 0 & 38 \\
\hline$T D C A$ & 0 & 0 & 0 & 0 & 0 & 0 & 0 & 0 & 0 & 0 & 0 & 0 & 0 & 0 & 0 \\
\hline$T C D C A$ & 47 & 16 & 135 & 6 & 0 & 48 & 2 & 0 & 21 & 0 & 0 & 17 & 6 & 0 & 19 \\
\hline
\end{tabular}

Data are presented as median $+[I Q R] .1^{\text {st }}$ column: median, $2^{\text {nd }}$ column: $25^{\text {th }}$ percentile and $3^{\text {rd }}$ column $75^{\text {th }}$ percentile.

Supplementary Table S5. Evolution of nutritional status before and during CR.

\begin{tabular}{|c|c|c|c|}
\hline Variable $(n=12)$ & Before CR & During CR & $P$ \\
\hline Energy intake, kcal/kg/day & 25.9 [20.9 to 28.4$]$ & 30.5 [27.9 to 36.8$]$ & .123 \\
\hline Protein intake, $\mathrm{g} / \mathrm{kg} /$ day & $1.0[0.9$ to 1.2$]$ & $1.4[1.0$ to 1.5$]$ & .069 \\
\hline Weight loss, \% & $10.9[4.5$ to 16.4$]$ & $9.9[5.4$ to 14.1$]$ & .161 \\
\hline BMI, $\mathbf{k g} / \mathrm{m}^{2}$ & 22.2 [20.2 to 25.8$]$ & 22.7 [20.3 to 25.4 & .754 \\
\hline Serum albumin, $g / L$ & 24.3 [20.1 to 29.3] & 32.3 [29.3 to 35.8$]$ & .002 \\
\hline Serum albumin $<30 \mathrm{~g} / \mathrm{L}, \mathrm{n}(\%)$ & $9(75)$ & $4(33)$ & .012 \\
\hline NRI & 71.6 [65.3 to 80.3$]$ & 84.7 [80.9 to 88.9$]$ & .017 \\
\hline $\mathrm{NRI}<83.5, \mathrm{n}(\%)$ & $11(92)$ & $4(33)$ & \multirow{3}{*}{.053} \\
\hline $83.5 \leq \mathrm{NRI}<97.5, \mathrm{n}(\%)$ & $1(8)$ & $8(67)$ & \\
\hline $97.5 \leq \mathrm{NRI} \leq 97.5, \mathrm{n}(\%)$ & $0(0)$ & $0(0)$ & \\
\hline
\end{tabular}

Data are median [IQR]. $P$ values in bold represent significant statistical differences. BMI indicates body mass index; NRI, nutritional risk index. 
Chapter 6

Study design

\begin{tabular}{|c|c|c|c|c|c|c|}
\hline \multicolumn{2}{|c|}{ Pre-Chyme reinfusion } & \multicolumn{5}{|c|}{ Chyme reinfusion period } \\
\hline & 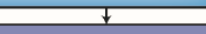 & $\downarrow$ & $\downarrow$ & $\downarrow$ & $\downarrow$ & $\downarrow$ \\
\hline Preparations & Day -3 & Day 0 & Day 7 & Day 21 & Day 35 & Day 49 \\
\hline $\begin{array}{l}\text { Patient recruitment } \\
\text { - Informed consent } \\
\text { Study enrollment } \\
\text { Preparations for medical } \\
\text { transfer }\end{array}$ & $\begin{array}{l}\text { - Blood collection } \\
\text { - Chyme collection } \\
\text { - Endoscopic biopsy } \\
\text { - Patient transfer to } \\
\text { Clinique St. Yves }\end{array}$ & $\begin{array}{l}\text { Blood } \\
\text { collection } \\
\text { Start CR }\end{array}$ & $\begin{array}{l}\text { Blood } \\
\text { collection }\end{array}$ & $\begin{array}{l}\text { Blood } \\
\text { collection } \\
\text { Endoscopic } \\
\text { biopsy }\end{array}$ & $\begin{array}{l}\text { Blood } \\
\text { collection }\end{array}$ & $\begin{array}{l}\text { Blood \& faeces } \\
\text { collection } \\
\text { Termination } \\
\text { of the study }\end{array}$ \\
\hline
\end{tabular}

Supplementary Figure S1. Study design. Overview of study design.

\section{S2-A}
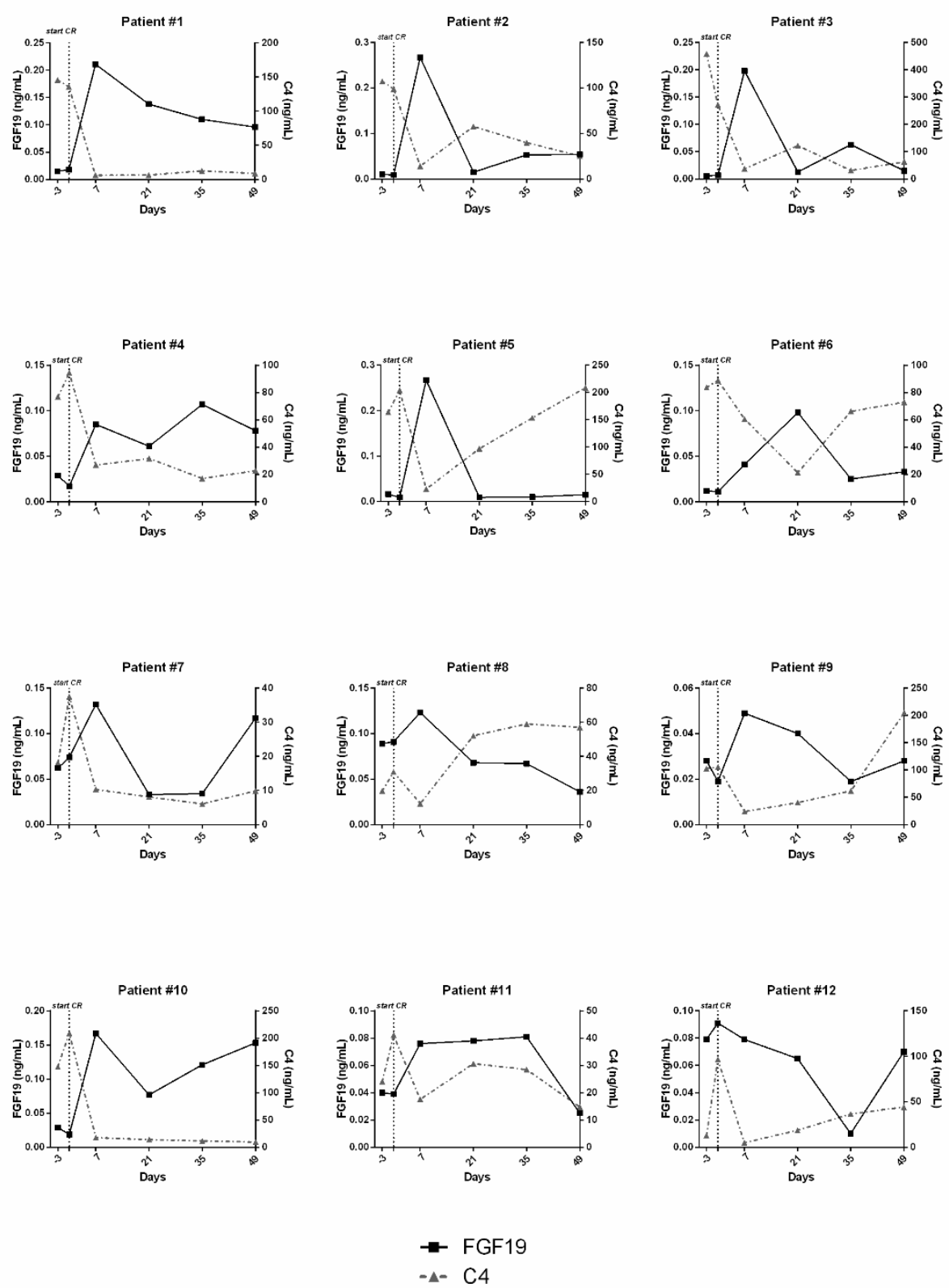
S2-B
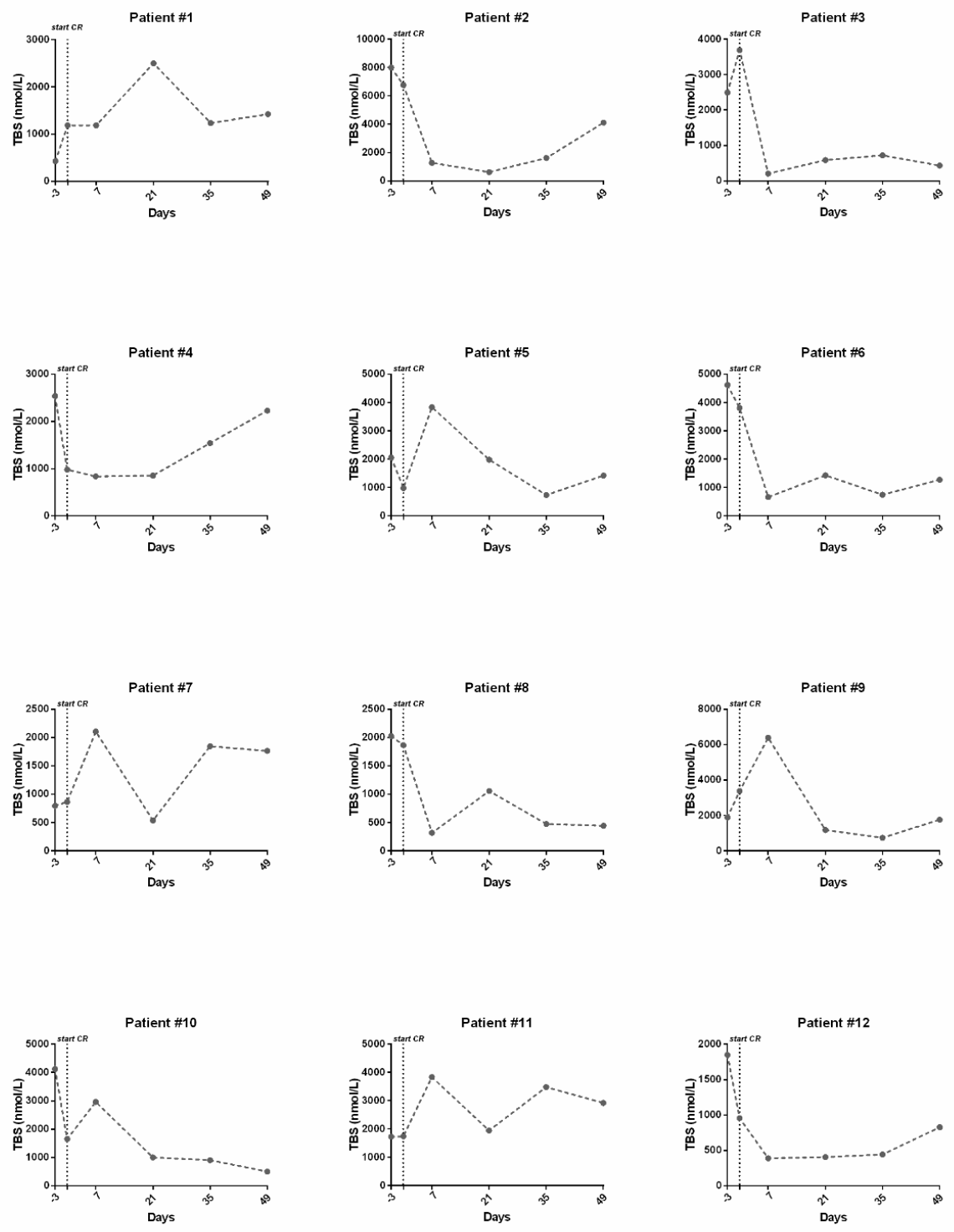

-• TBS

Supplementary Figure S2A-B. Individual plasma FGF19, C4 and TBS time courses. Individual plasma time courses of (A) FGF19, C4 and (B) TBS in all twelve patients. In (A) the interrupted line depicts FGF19 and the black line C4 levels. 
A

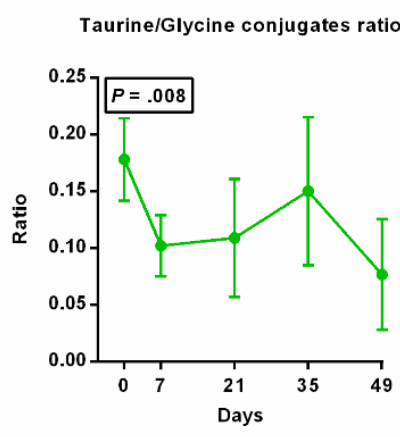

C

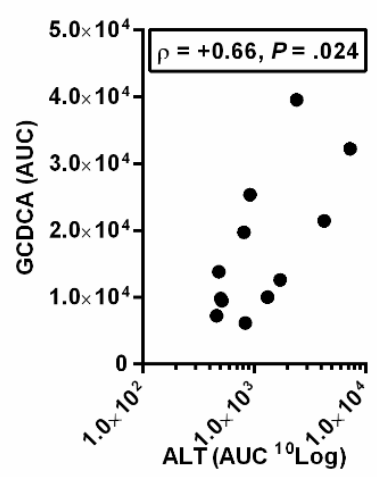

B
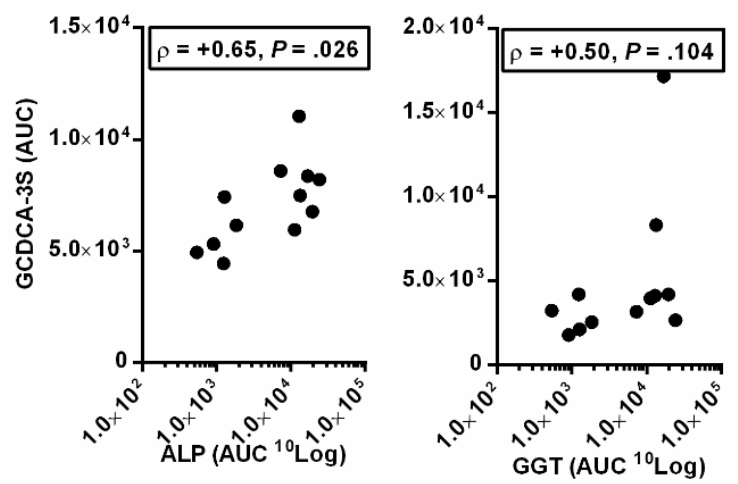

Supplementary Figure S3. Ratio of taurine- to glycine-conjugated bile salts and association of GCDCA-3S with liver injury markers. Time course of $(\mathbf{A})$ the ratio of taurine- to glycine-conjugated bile salts and (B) correlation between GCDCA-3S and ALP and GGT (area under the curve). (C) Correlation between GCDCA-3S, GCA, TCA and ALT levels (area under the curve). Trends in time were evaluated by ANOVA with repeated measures. Correlations were evaluated with Spearman's $(\rho)$ correlation coefficient. $P$ values are depicted. 

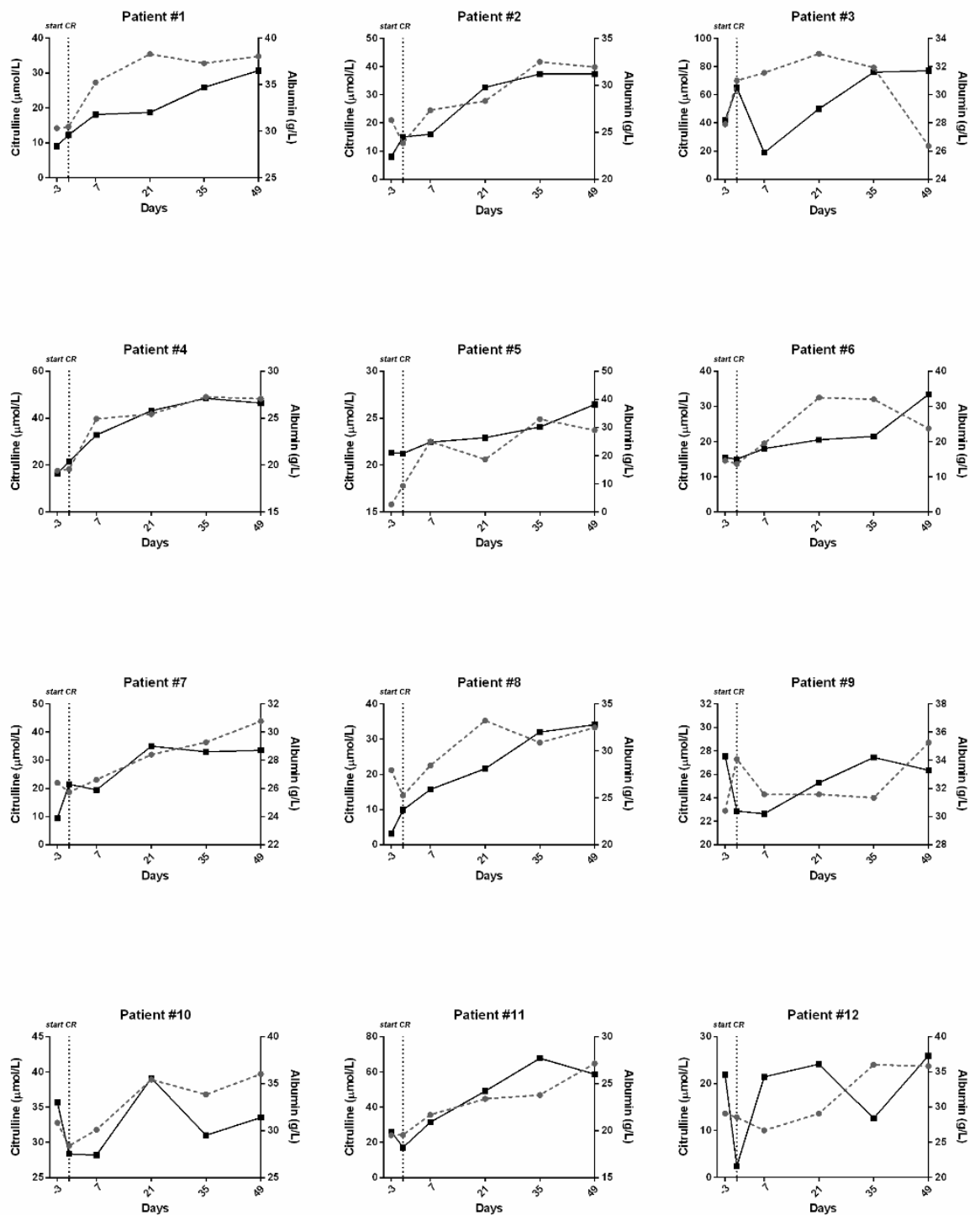

-๑) CIT

$\rightarrow$ Albumin

Supplementary Figure S4. Individual plasma CIT and albumin plasma time courses. Individual plasma time courses of CIT and albumin of all patients. The interrupted line depicts CIT and the black line albumin levels. 
Chapter 6

IL-8

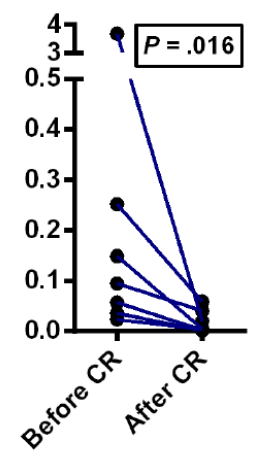

IL-17

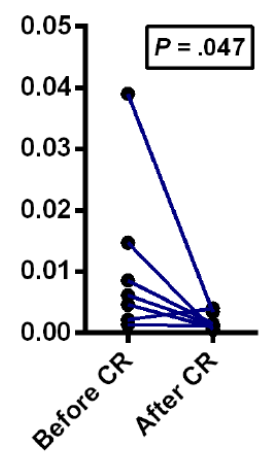

Supplementary Figure S5. mRNA expression of IL-8 and IL-17. mRNA expression of pro-inflammatory cytokines $I L-8$ and $I L-17$. $P$ values are depicted.

ALT

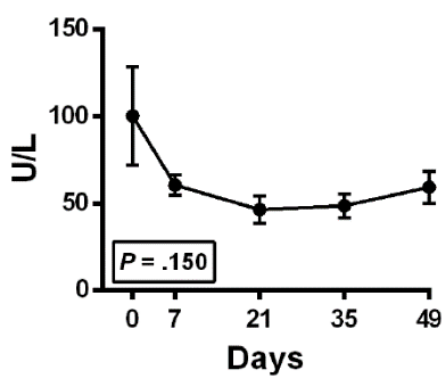

AST

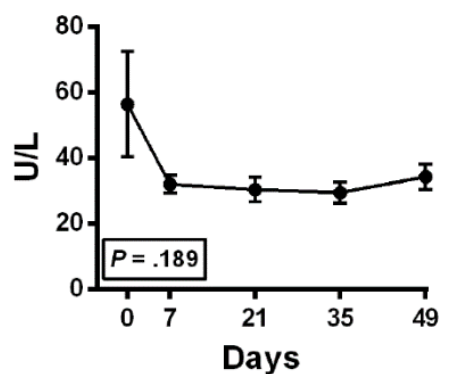

Supplementary Figure S6. Liver injury markers plasma time courses. Plasma time courses of ALT and AST. Trends in time were evaluated by ANOVA with repeated measures. $P$ values are depicted. 




\section{Chapter 7}

General discussion 
A disrupted enterohepatic circulation (EHC) of bile salts leads to unfavorable outcomes in surgical patients ${ }^{1,2}$. Patients with hyperbilirubinemia due to biliary obstruction have a high rate of severe complications after partial liver resection ${ }^{3}$. In addition, due to the loss of bile salts (i.e. liver and/or gallbladder tissue) and diminished bile salt signaling, patients undergoing partial hepatectomy are at risk of liver failure and death, especially in case of a small future liver remnant $(F L R)^{4}$. Furthermore, patients with an interrupted EHC due to a temporary jejunal double enterostomy can develop intestinal failure (IF) and intestinal failure associated liver disfunction (IFALD) because of loss of succus entericus ${ }^{5}$. To improve clinical outcomes, various treatments targeting the circulation and signaling function of bile salts are employed/tested in surgical patients or experimental models, including preoperative biliary drainage (BD) ${ }^{6}$, bile salt receptor activation ${ }^{7,8}$, and chyme reinfusion ${ }^{5}$.

The central aim of the current thesis is to investigate the role of restored or activation of the enterohepatic bile salt signaling axis, as well as consequences of cholestasis on liver regeneration and intestinal failure (secondary to a temporary double enterostomy). This translated into five main specific aims: 1) to assess the effect and optimal route of preoperative biliary drainage in patients with resectable pCCA (Chapter 2); 2) to explore precision-cut liver slices (PCLS) as a model for studying factors affecting human liver regeneration ex vivo (Chapter 3 ); 3 ) to investigate the influence of cholestasis on portal vein embolization (PVE)-induced FLR hypertrophy in patients (Chapter 4); 4) to study the mechanism of obeticholic acid (OCA)-augmented hypertrophy in experimental PVE (Chapter 5); and 5) to study the contribution of the bile salt-fibroblast growth factor 19 (FGF19) axis in intestinal failure patients receiving chyme reinfusion (Chapter $\mathbf{6})$.

\section{Preoperative biliary drainage for resectable perihilar cholangiocarcinoma}

Preoperative $\mathrm{BD}$ is used to decompress the biliary tree and improve FLR function before liver resection. However, the value of preoperative $\mathrm{BD}$ in resectable pCCA is under debate. Additionally, the best drainage route (percutaneous transhepatic biliary drainage, PTBD or endoscopic biliary drainage, EBD) still needs to be determined. In Chapter 2, we conducted a systematic review of systematic reviews (SRs) to assess the effect of preoperative $B D$ versus no preoperative $B D$, and the superiority of EBD versus $\mathrm{PTBD}$, on perioperative and long-term outcomes in patients with resectable 
pCCA. The results indicate that preoperative BD might be beneficial in strictly selected patients. In addition, EBD might be associated with higher short-term drainage-related morbidity (e.g. cholangitis and pancreatitis), but more favorable long-term survival. It should be emphasized that all but one of the original studies included in the respective SRs had a retrospective design, which could not avoid confounding factors in the study period. Furthermore, most included SRs had a high risk of bias according the ROBIS assessment tool. Notably, there is considerable variability in the inclusion criteria of original studies and the numbers of participants and events abstracted across the SRs. These differences could be caused by variable periods of study inclusion, inclusion criteria, and data extraction errors. Errors in the data extraction procedure are an important source of bias in SRs ${ }^{9}$. The influence of risk of bias on pooled results could lead to under- or overestimation of the actual intervention effects, resulting in a limitation of the validity of drawn conclusions ${ }^{10}$.

The one randomized controlled trial (RCT) conducted thus far, demonstrated higher mortality in the PTBD compared to the EBD group ${ }^{11}$. PTBD increased perioperative mortality in patients with resectable pCCA, with 3 of 11 fatalities occurring before surgery ${ }^{11}$. This study was prematurely stopped because of higher mortality in the PTBD group. A possible reason is that externally draining bile in the PTBD procedure results in an impaired contribution of bile salt signaling to the regenerative response and processes. Future studies to compare PTBD with reinfusion of bile fluid, to EBD in patients with pCCA are recommended.

In addition, further studies should pay attention to the differences of internal or external biliary drainage on perioperative and postoperative outcomes in resectable pCCA. Because internal drainage elicits a different physiological response compared to external drainage that abrogates the digestive, signaling and antimicrobial roles of bile salts. Abated activation of ileal farnesoid $X$ receptor $(F X R)$ can result in the loss of negative feedback control of hepatic bile salt synthesis and bile salt overload with attendant hepatotoxicity when export to the biliary network is hampered ${ }^{1}$. In the setting of external drainage, it can be considered to give bile back orally or via a naso-gastric tube, to allow beneficial antimicrobial and signaling actions of bile salts. ${ }^{12-14}$.

\section{PCLS as an in vitro model to study human liver regeneration}


Liver regeneration requires the temporally orchestrated proliferation of hepatocytes as well all the other cell types that support liver function, such as vascular endothelial cells, innate immune cells, and bile duct-lining cholangiocytes ${ }^{15}$, 16 . Paracrine signaling via soluble factors (e.g. angiocrine factors) is pivotal in this process, and restoration of the livers' functional architecture ${ }^{17}$. As an ex vivo model, PCLS contains various parenchymal and non-parenchymal cells, imitating liver original environment ${ }^{18}$. PCLS may be a good platform to study the cell-cell interactions in liver regeneration, and test strategies to augment liver growth.

In Chapter 3, we used FGF19 and bile salt nuclear receptors agonists to test the suitability of human PCLS (hPCLS) as an in vitro model to study liver regeneration. The responsiveness of hPCLS to FGF19 could not be verified at the level of ERK1/2, a kinase that is activated after binding of FGF19 to its receptor FGFR4. ERK1/2 activation (phosphorylated form) was already maximal without addition of FGF19. This likely results from external triggers during harvesting or processing of tissue for hPCLS preparation. Unfortunately, the expression of FGF19-regulated genes could not be used as a read-out of FGF19 activity, as CYP7A1 became undetectable after $>19$ hrs of culturing and PCK1 was drastically reduced. Since we could not verify FGF19 responsiveness in hPCLS, we cannot draw a conclusion on the role of FGF19 in driving hepatocellular proliferation in humans.

Constitutive androstane receptor (CAR) activation by CITCO (6-(4Chlorophenyl)imidazo[2,1-b][1,3]thiazole-5-carbaldehyde O-(3,4dichlorobenzyl)oxime) was reported to induce hepatocyte proliferation in PCLS, including slices from the liver of one patient 19. Despite carefully replicating experimental conditions, however, we could not reproduce the proliferative effect of CITCO in hPCLS derived from three distinct patients. Because induction of CAR target gene CYP2E1 by CITCO was not observed in hPCLS, we cannot draw a conclusion on CAR action.

In its current form, the hPCLS model is not suitable for studying the role of FGF19 on liver regeneration. Further measures including systematic evaluation of patient factors (background liver disease, prior chemotherapy, ischemic time, etc), and culturing conditions (e.g. optimized recovery time, strategies to prevent dedifferentiation etc.) should be considered in optimizing the hPCLS as model to study -and therapeutically modulate- liver regeneration. 


\section{Bile salt homeostasis on PVE-induced liver regeneration}

The FXR agonist OCA accelerated liver regeneration following PVE, reflected by increased hepatic volume and proliferation in the non-embolized lobe in a standardized rabbit model ${ }^{7}$. However, the underlying mechanism has not been defined yet. In Chapter 4 we aim to reveal the mechanisms underlying accelerated FLR growth in OCA-treated rabbit undergoing PVE. We observed earlier normalization of serum total bile salt levels after PVE in OCA-treated animals. In the non-embolized lobe, bile salts levels were lower upon OCA treatment, which may be caused by reduced bile salt synthesis combined with enhanced basolateral export of bile salts. Serum bile salt composition profile showed no major shift by OCA treatment. However, the absolute levels of lithocholic acid (LCA, a cytotoxic bile salt) and hyodeoxycholic acid (HDCA) were lower in OCA-treated animals, and their levels were negatively correlated with FLR hypertrophy. Furthermore, transcript profiling by RNA sequencing and subsequent pathway analysis of differentially expressed genes, provided support for an important role of bile salt homeostasis to accelerate FLR hypertrophy. Additional candidates for mediating growth-stimulating effects of OCA include the secreted factors bone morphogenetic protein 3 (Bmp3) and Indian hedgehog (Ihh). This study indicates that improved bile salt homeostasis underlies the increased hypertrophy of the nonembolized liver lobe in OCA-treated animals.

In the clinical setting, patients often have damaged liver parenchyma and function before the PVE procedure, such as cholestasis, chemotherapy-associated liver injury (CALI) and liver fibrosis. Impaired liver function is associated with subsequent attenuated liver regeneration after PVE ${ }^{20}$. Treatment with OCA may have potential to accelerate FLR volume gain in patients before liver resection. This can be beneficial to shorten the interval between PVE and liver resection, thus decreasing the risk of tumor progression (and degree of CALI) during this interval. OCA has been shown to improve hepatic fibrosis and reduce blood cholestatic markers in patients with chronic liver diseases in recent clinical trials ${ }^{21,22}$. A large body of safety data in humans is available for OCA from phase II-IV trials ${ }^{21-23}$ and thus application of OCA in surgical patients for inducing liver regeneration is promising. However, an essential additional preclinical step would be the demonstration of lack of effects of OCA on tumor progression in the embolized segments. Once optimized, hPCLS derived from tumoral liver tissue may be usable to study this aspect in a human model system. 
The role of bile salt homeostasis on liver growth in the rabbit model of PVE led us to investigate the influence of cholestasis on FLR hypertrophy in patients undergoing PVE. Thus, in Chapter 5, we retrospectively analyzed data from 50 patients (31 colorectal liver metastases (CRLM), 19 pCCA) who underwent PVE before major liver resection in Maastricht University Medical Center (Maastricht, The Netherlands) or the Uniklinik RWTH Aachen (Aachen, Germany). We found that FLR volumes increased after PVE in both patients with CRLM (all non-cholestatic) and pCCA (majority still cholestatic despite being drained). The degree of hypertrophy and kinetic growth rate, two measures reflecting FLR hypertrophy induced by PVE, were not different between patients with pCCA and CRLM. Patients with pCCA are routinely drained prior to resection, and also here the majority of these patients were drained prior to PVE. After drainage, pCCA patients with or without hyperbilirubinemia had comparable indices of FLR hypertrophy. Furthermore, neither serum bilirubin levels before drainage, nor before PVE were correlated with indices of FLR hypertrophy.

Because blood samples were not available in this retrospective cohort, we could not determine serum levels of bile salt homeostasis-related molecules, such as total and individual bile salts, FGF19, and C4 (a marker of synthesis of bile salt). As mentioned in Chapter 4, bile salts play an important role in liver regeneration via activation of the nuclear receptor FXR in the ileum and the liver. Future prospective studies with longitudinal collection of blood samples are recommended to further assess the association of the bile salt-FGF19 axis with FLR hypertrophy in patients undergoing PVE.

Through analyzing the impact of uni- vs. bilateral biliary drainage on FLR hypertrophy, we found that unilateral drainage of FLR induced higher $\mathrm{DH}$ than bilateral drainage. This finding suggests that unilateral drainage might be superior when performing initial biliary drainage. In addition, elevated inflammatory response (increased C-reactive protein levels) was associated with impaired liver growth in patients with pCCA. Serum C-reactive protein alterations may be promising to be used as a surrogate marker of PVE-induced FLR hypertrophy in patients with pCCA. Due to limited number of patients in our study, however, studies with larger number of participants are required to substantiate this notion.

\section{Chyme reinfusion and bile salt-FGF19 axis}


Enterocutaneous fistulae or surgically created (small intestinal) enterostomies disrupt the enterohepatic circulation and can result in (functional) intestinal failure ${ }^{5}$. Several retrospective studies showed that chyme reinfusion $(C R)$ can improve nutritional status, intestinal absorptive function, and reverse liver injury, in patients with acute intestinal failure (IF) ${ }^{5,24,25}$. In Chapter 6 we describe a prospective study with a pre- and postintervention design to investigate a potential contribution of the bile salt-FGF19 axis to the beneficial actions of $\mathrm{CR}$ in intestinal failure patients with a temporary double enterostomy (RESCUE study).

Disturbance of the bile salt-FGF19 axis in these patients was evident at baseline (prior to $\mathrm{CR}$ ), as judged from low serum FGF19 and marked elevation of serum C4. CR induced a prompt increase in plasma FGF19 and decrease in C4 levels, indicating restored regulation of bile salt synthesis via endocrine FGF19 action. Fractions of secondary and deconjugated bile salts were increased after $\mathrm{CR}$, reflecting restored contact between host bile salts and the colonic flora, allowing microbial bile salt metabolism. Furthermore, citrulline and albumin levels were gradually rising after $C R$, while abnormal serum liver tests normalized after $\mathrm{CR}$, indicating restored intestinal function, improved nutritional status and amelioration of liver injury. CR increased ileal gene transcripts related to enterocyte number, and carbohydrate and bile salt handling. Finally, the reciprocal FGF19/C4 response after 7 days, predicted the plasma citrulline time course, indicating beneficial effects of restored hepatic synthesis of bile salt on improved intestinal function.

Remarkably, our study showed that jejunal output prior to $C R$ was strongly related to systemic C4 levels. A plausible explanation could be that enhanced synthesis and biliary secretion of bile salts induces a choleretic effect and eventually elevated jejunal output ${ }^{26}$. In support of such notion, patients with chronic primary bile salt diarrhea have high C4 levels ${ }^{27}$.

The findings of this study make it conceivable that the beneficial effects of CR are mediated in part through a restored bile salt-FGF19 signaling axis. In clinics, CR need to be considered in surgical patients with a distal entrance of the small bowel in case IF develops. If the effluent part of the small intestine cannot be accessed for $C R$, systemic FXR/FGF19-based therapy could be considered. Engineered FGF19 variants were already shown to be effective in lowering bile salt synthesis in patients with cholestatic or metabolic liver disease ${ }^{28,29}$. 


\section{Future perspective}

Due to a relatively low incidence of $\mathrm{pCCA}$, long-running RCTs with large sample sizes are lacking to provide strong evidence for the use (or not) of preoperative BD. Therefore, international multicenter cooperation is urgently needed to settle this medical question. The main issue in the current clinical practice is that uniform indications for preoperative BD are lacking in pCCA. This can be considered from at least three perspectives. Firstly, a serum bilirubin threshold needs to be determined for $\mathrm{BD}$. Additionally, cholangitis needs to be considered because of its association with increased mortality in resectable pCCA ${ }^{4}$. Furthermore, small FLR can be an indication for preoperative $\mathrm{BD}$. Because preoperative $\mathrm{BD}$ did not increase major morbidity after liver resection in jaundiced patients with small FLR ${ }^{30}$.

In initial explorations of suitability of hPCLS as a model system to study liver regeneration, we observed rapid loss of CYP7A1 mRNA expression to undetectable levels after $19 \mathrm{~h}$ of incubation. As CYP7A1 expression is used as a readout for FGF19 signaling, optimization of culturing conditions such that a differentiated hepatocyte phenotype is maintained, is necessary to draw conclusions on FGF19 action on liver growth. In addition, RNA sequencing, phosphoproteome analysis, and metabolite profiling by mass spectrometry, can be used to find yet undefined targets of FGF19 action in hPCLS.

We retrospectively analyzed the influence of cholestasis on FLR hypertrophy after PVE through comparing patients with CRLM (all non-cholestatic) and PCCA (predominantly cholestatic)., Direct study of molecules that pertain to bile salt homeostasis in plasma and/or non-embolized liver tissue of clinical patients before and after PVE, can provide deeper insights into factors influencing PVE-induced liver hypertrophy. Bile salts levels at $5 \mathrm{~h}$ after PVE have been reported to be positively correlated with the increase in FRL volume in patients with CRLM ${ }^{31}$. However, the downstream pathways mediating FLR hypertrophy remain ill-defined. In addition, the impact of local inflammation on FLR growth should be investigated in patients and/or an optimized hPCLS model . Besides PVE, simultaneous embolization of portal and hepatic veins (PVE/HVE) is a new approach to induce FLR growth. Notably, PVE/HVE achieved better FLR hypertrophy and resectability than PVE alone ${ }^{32}$. It will be interesting to explore the potential role of bile salt signaling in PVE/HVE induced liver growth.

In the RESCUE study, we observed improved intestinal and liver function by the enteral 
nutrition technique of chyme reinfusion, resulting in restored continuity of the EHC in intestinal failure patients with abnormal liver tests. As recommended by the European Society for Clinical Nutrition and Metabolism (ESPEN), oral or enteral nutrition (including distal enteral tube feeding) should be established in patients with intestinal failure-associated liver disease ${ }^{33}$. In addition, our study suggests that when patients need to undergo a temporary proximal enterostomy (e.g. during acute mesenteric ischemia), establishment of a double-barreled enterostomy or placement of a distal entrance of the small bowel should be considered in management of patients. Further, a plausible suggestion of our study is that (intramuscular) administration of FGF19 analogues could be beneficial to improve gut-liver function in intestinal failure patients when there is no accessible distal small bowel. 


\section{References}

1. Schaap FG, Trauner M, Jansen PL. Bile acid receptors as targets for drug development. Nat Rev Gastroenterol Hepatol. 2014;11:55-67.

2. Albillos A, de Gottardi A, Rescigno M. The gut-liver axis in liver disease: Pathophysiological basis for therapy. J Hepatol. 2020;72:558-77.

3. Wronka KM, Grat M, Stypulkowski J, Bik E, Patkowski W, Krawczyk M, et al. Relevance of Preoperative Hyperbilirubinemia in Patients Undergoing Hepatobiliary Resection for Hilar Cholangiocarcinoma. J Clin Med. 2019;8:458.

4. Olthof PB, Wiggers JK, Groot Koerkamp B, Coelen RJ, Allen PJ, Besselink MG, et al. Postoperative Liver Failure Risk Score: Identifying Patients with Resectable Perihilar Cholangiocarcinoma Who Can Benefit from Portal Vein Embolization. J Am Coll Surg. 2017;225:387-94.

5. Picot D, Layec S, Dussaulx L, Trivin F, Thibault R. Chyme reinfusion in patients with intestinal failure due to temporary double enterostomy: A 15-year prospective cohort in a referral centre. Clin Nutr. 2017;36:593-600.

6. Inamdar S, Slattery E, Bhalla R, Sejpal DV, Trindade AJ. Comparison of Adverse Events for Endoscopic vs Percutaneous Biliary Drainage in the Treatment of Malignant Biliary Tract Obstruction in an Inpatient National Cohort. JAMA Oncol. 2016;2:112-7.

7. Olthof PB, Huisman F, Schaap FG, van Lienden KP, Bennink RJ, van Golen RF, et al. Effect of obeticholic acid on liver regeneration following portal vein embolization in an experimental model. Br J Surg. 2017;104:590-9.

8. Huang W, Ma K, Zhang J, Qatanani M, Cuvillier J, Liu J, et al. Nuclear receptor-dependent bile acid signaling is required for normal liver regeneration. Science. 2006;312:233-6.

9. Onasanya O, lyer G, Lucas E, Lin D, Singh S, Alexander GC. Association between exogenous testosterone and cardiovascular events: an overview of systematic reviews. The Lancet Diabetes \& Endocrinology. 2016;4:943-56.

10. Higgins JP, Altman DG, Gotzsche PC, Juni P, Moher D, Oxman AD, et al. The Cochrane Collaboration's tool for assessing risk of bias in randomised trials. BMJ. 2011;343:d5928.

11. Coelen RJS, Roos E, Wiggers JK, Besselink MG, Buis Cl, Busch ORC, et al. Endoscopic versus percutaneous biliary drainage in patients with resectable perihilar cholangiocarcinoma: a multicentre, randomised controlled trial. Lancet Gastroenterol Hepatol. 2018;3:681-90.

12. Yoshida Y, Ajiki T, Ueno K, Shinozaki K, Murakami S, Okazaki T, et al. Preoperative bile replacement improves immune function for jaundiced patients treated with external biliary drainage. J Gastrointest Surg. 2014;18:2095-104.

13. Thompson JN, Cohen J, Moore RH, Blenkharn JI, McConnell JS, Matkin J, et al. Endotoxemia in obstructive jaundice. Observations on cause and clinical significance. Am $\mathrm{J}$ Surg. 1988;155:314-21.

14. Tripathy U, Dhiman RK, Attari A, Katariya RN, Ganguly NK, Chawla YK, et al. Preoperative bile salt administration versus bile refeeding in obstructive jaundice. Natl Med J India. 1996;9:66-9.

15. Forbes SJ, Newsome PN. Liver regeneration - mechanisms and models to clinical application. Nat Rev Gastroenterol Hepatol. 2016;13:473-85.

16. Tirnitz-Parker JEE, Forbes SJ, Olynyk JK, Ramm GA. Cellular Plasticity in Liver Regeneration: Spotlight on Cholangiocytes. Hepatology. 2018.

17. Ding BS, Cao Z, Lis R, Nolan DJ, Guo P, Simons M, et al. Divergent angiocrine signals from 
vascular niche balance liver regeneration and fibrosis. Nature. 2014;505:97-102.

18. de Graaf IA, Olinga P, de Jager MH, Merema MT, de Kanter R, van de Kerkhof EG, et al. Preparation and incubation of precision-cut liver and intestinal slices for application in drug metabolism and toxicity studies. Nat Protoc. 2010;5:1540-51.

19. Tschuor C, Kachaylo E, Limani P, Raptis DA, Linecker M, Tian Y, et al. Constitutive androstane receptor (Car)-driven regeneration protects liver from failure following tissue loss. J Hepatol. 2016;65:66-74.

20. Kageyama Y, Kokudo T, Amikura K, Miyazaki Y, Takahashi A, Sakamoto H. Impaired liver function attenuates liver regeneration and hypertrophy after portal vein embolization. World $\mathrm{J}$ Hepatol. 2016;8:1200-4

21. Hirschfield GM, Mason A, Luketic V, Lindor K, Gordon SC, Mayo M, et al. Efficacy of obeticholic acid in patients with primary biliary cirrhosis and inadequate response to ursodeoxycholic acid. Gastroenterology. 2015;148:751-61.e8.

22. Neuschwander-Tetri BA, Loomba R, Sanyal AJ, Lavine JE, Van Natta ML, Abdelmalek MF, et al. Farnesoid $X$ nuclear receptor ligand obeticholic acid for non-cirrhotic, non-alcoholic steatohepatitis (FLINT): a multicentre, randomised, placebo-controlled trial. Lancet. 2015;385:956-65.

23. Kowdley KV, Vuppalanchi R, Levy C, Floreani A, Andreone P, LaRusso NF, et al. A randomized, placebo-controlled, phase II study of obeticholic acid for primary sclerosing cholangitis. J Hepatol. 2020.

24. Lévy E PD, Frileux P, Parc R, Huguet C, Loygue J. Inhibition of upper gastrointestinal secretions by reinfusion of succus entericus into the distal small bowel. A clinical study of 30 patients with peritonitis and temporary enterost. Ann Surg. 1983;198:596-600.

25. Picot D, Garin L, Trivin F, Kossovsky MP, Darmaun D, Thibault R. Plasma citrulline is a marker of absorptive small bowel length in patients with transient enterostomy and acute intestinal failure. Clin Nutr. 2010;29:235-42.

26. Erlinger S, Dhumeaux D. Mechanisms and control of secretion of bile water and electrolytes. Gastroenterology. 1974;66:281-304.

27. Walters JR, Johnston IM, Nolan JD, Vassie C, Pruzanski ME, Shapiro DA. The response of patients with bile acid diarrhoea to the farnesoid $X$ receptor agonist obeticholic acid. Aliment Pharmacol Ther. 2015;41:54-64.

28. Hirschfield GM, Chazouilleres O, Drenth JP, Thorburn D, Harrison SA, Landis CS, et al. Effect of NGM282, an FGF19 analogue, in primary sclerosing cholangitis: A multicenter, randomized, double-blind, placebo-controlled phase II trial. J Hepatol. 2019;70:483-93.

29. Harrison SA, Neff G, Guy CD, Bashir MR, Paredes AH, Frias JP, et al. Efficacy and Safety of Aldafermin, an Engineered FGF19 Analog, in a Randomized, Double-Blind, Placebo-Controlled Trial of Patients With Nonalcoholic Steatohepatitis. Gastroenterology. 2020.

30. Mehrabi A, Khajeh E, Ghamarnejad O, Nikdad M, Chang DH, Buchler MW, et al. Meta-analysis of the efficacy of preoperative biliary drainage in patients undergoing liver resection for perihilar cholangiocarcinoma. Eur J Radiol. 2020;125:108897.

31. Hoekstra LT, van Lienden KP, Schaap FG, Chamuleau RA, Bennink RJ, van Gulik TM. Can plasma bile salt, triglycerides, and apoA-V levels predict liver regeneration? World $\mathrm{J}$ Surg. 2012;36:2901-8.

32. Heil J, Korenblik R, Heid F, Bechstein WO, Bemelmans M, Binkert C, et al. Preoperative portal 


\section{Chapter 7}

vein or portal and hepatic vein embolization: DRAGON collaborative group analysis. Br J Surg. 2021.

33. Lal S, Pironi L, Wanten G, Arends J, Bozzetti F, Cuerda C, et al. Clinical approach to the management of Intestinal Failure Associated Liver Disease (IFALD) in adults: A position paper from the Home Artificial Nutrition and Chronic Intestinal Failure Special Interest Group of ESPEN. Clin Nutr. 2018;37:1794-7. 




\section{Appendix I}

Summary 

Bile salts not only aid in absorption of dietary lipids, but also act as signaling molecules controlling negative feedback regulation of synthesis of bile salts, lipogenesis, and gluconeogenesis in the liver. The bile salt-fibroblast growth factor 19 (FGF19) signaling axis depends on an intact enterohepatic circulation $(\mathrm{EHC})$ of bile salts, and is important for gut-liver health including promoting liver regeneration and improving intestinal failure (IF) and intestinal failure-associated liver disease (IFALD).

In Chapter 1 we provided a general introduction to this thesis. We first introduced the physiology of the EHC of bile salts, the impact of biliary obstruction (e.g. perihilar cholangiocarcinoma, $\mathrm{pCCA}$ ) on the $\mathrm{EHC}$, and the interventional strategies (i.e. preoperative biliary drainage, $\mathrm{BD}$ ) to decompress the biliary obstruction. Furthermore, we discussed the interrupted EHC due to loss of the 'source' of bile salts, such as partial hepatectomy, and the potential role of bile salt signaling on portal vein embolization (PVE)-induced liver hypertrophy. Moreover, we introduced disturbance of the EHC because of outflow of succus entericus in the stoma bag in patients with a temporary jejunal double enterostomy, and beneficial effects of chyme reinfusion (CR) in improving IF and IFALD. Finally, the aim and outline of this thesis were summarized.

In Chapter 2 we gave a systematic review of systematic reviews to assess the effect and route of preoperative BD in patients with resectable perihilar cholangiocarcinoma (pCCA). Through searching database, eleven systematic reviews (SRs) with metaanalysis including 5950 patients were identified. All but one original studies in the SRs were retrospective. Ten of eleven SRs had high risk of bias. For preoperative BD versus no preoperative $\mathrm{BD}$, all SRs showed no statistical differences in postoperative mortality. Preoperative BD was associated with increased postoperative major morbidity in 'simple criteria' patients receiving BD only based on the presence of jaundice. For endoscopic biliary drainage (EBD) versus percutaneous transhepatic biliary drainage (PTBD), three of four SRs showed that the postoperative mortality was not significantly different between the two drainage modes. EBD was associated with higher drainage-related overall morbidity, cholangitis and pancreatitis rates in three of four, three of five, and four of four SRs, respectively. PTBD was associated with higher seeding metastasis rates and worse postoperative overall survival. This study does not end the debate on the significance and preferred route of preoperative BD. The preoperative $\mathrm{BD}$ might need to be performed in strictly selected patients in terms of cholangitis, bilirubin levels and future liver remnant volume to avoid increased postoperative major morbidity. EBD might be associated with higher short-term 
drainage-related morbidity but more favorable long-term oncological outcomes.

In Chapter 3 we explored the suitability of human precision-cut liver slices (hPCLS) as an in vitro model to study human liver regeneration. hPCLS were derived from tumordistal liver tissue from patients who underwent partial liver resection for colorectal liver metastases (CRLM). hPCLS were incubated with lipopolysaccharide (LPS), recombinant FGF19, and nuclear bile salt receptor agonists (obeticholic acid [OCA], farnesoid X receptor [FXR] agonist; 6-(4-Chlorophenyl)imidazo[2,1-b][1,3]thiazole-5carbaldehyde O-(3,4-dichlorobenzyl)oxime [CITCO], constitutive androstane receptor [CAR] agonist; rifampicin, pregnane $X$ receptor [PXR] agonist). $5 \mathrm{C}$ and Geltrex were used to assess their effectiveness in maintaining expression of genes related to a differentiated hepatocyte phenotype. mRNA expression of IL6, FXR/FGF19 pathwayrelated genes and proliferation-related genes were assessed. We found that FGF19 did not induce IL6 mRNA expression in hPCLS. In addition, marked phosphorylation of extracellular signal-related kinase (ERK)1/2 was already observed in untreated liver slices. FGF19 had no additional effect on levels of phosphorylated ERK1/2. Expression of FGF19 pathway-related genes (CYP7A1, FGFR4, KLB, PCK1) rapidly declined upon $\mathrm{hPCLS}$ culturing. The use of $5 \mathrm{C}$ and Geltrex did not maintain expression of these genes during culturing of hPCLS. Furthermore, nuclear receptors agonists OCA and rifampicin did not induce cell proliferation in hPCLS. The efficacy of FGF19 and CAR treatment could not be ascertained in these exploratory experiments. Hence, questions regarding the involvement of the FGF19/IL6 axis in hepatocellular proliferation, and augmentation of liver growth by CAR agonism could not be answered with the current method of hPCLS source, preparation and culturing.

In Chapter 4 we investigated the mechanisms by which obeticholic acid (OCA) accelerates liver hypertrophy following PVE in a rabbit model. Specimen were analyzed from a previous experiment in which rabbits were treated by oral gavage with OCA (10 mg/kg, $\mathrm{n}=11$ ) or vehicle (control group, $\mathrm{n}=11$ ) prior to, and during the course of, PVE. Serum total bile salts (TBS), bile salt composition, C4 (marker of bile salt synthesis), and liver synthetic function were determined before, after $3 \mathrm{hrs}$, and at day 1 , day 3, and day 7 after PVE. lleal and hepatic genes expression were analyzed through targeted and untargeted approaches at day three after PVE, when the growthenhancing effect of OCA was maximal. For correlation analyses, volumes of (non)embolized liver segments, and \%volume gain, were previously determined by CT scan. We previously reported that PVE induced a larger volume gain of the non- 
embolized liver lobe in OCA-treated animals. Effects of OCA were largest at 3 days after PVE (volume gain: $56.1 \%$ vs. $26.1 \%$ in OCA- and vehicle-treated animals, respectively). OCA treatment led to earlier normalization of serum TBS levels and lower bile salt content of the non-embolized liver lobe after PVE. OCA decreased mRNA expression of Cyp7a1 (involved in bile salt synthesis) and promoted expression of S/c51a/b (involved in bile salt export) in the non-embolized liver lobe, likely contributing to the observed improved serum and liver bile salt homeostasis. Levels of lithoholic acid (LCA), a hepatotoxic bile salt, were lower in the OCA-treated animals compared to the control animals at day 1 and day $3(P=0.002$ and $P=0.031$, respectively). LCA levels were negatively related with caudal (i.e. non-embolized) liver volume (CLV) increase and the numbers of Ki-67 positive hepatocytes in this lobe ( $\rho=$ $-0.726, \mathrm{P}<0.001 ; \rho=-0.718, \mathrm{P}=0.009$, respectively). Both targeted and untargeted transcript analysis revealed associations with genes engaged in bile salt homeostasis and liver growth, adding to the notion that OCA-mediated improvement of bile salt homeostasis was in part responsible for enhanced PVE-induced liver growth. Additional factors candidates for mediating growth-stimulating effects of OCA include the secreted factors Bmp3 and Ihh. Our findings indicate that OCA improved bile salt homeostasis, which partly contributed to augmented hypertrophy of the non-embolized liver lobe in rabbits. OCA may be promising to increase the efficacy of PVE in patients with small future liver remnant planned for (extended) partial liver resection.

In Chapter 5 we assessed the influence of cholestasis on PVE-induced future liver remnant (FLR) hypertrophy. Patients were enrolled with pCCA or CRLM, who underwent PVE before a (extended) right hemihepatectomy on 2016-2019. Volume of segments II and III were considered as FLR and assessed on pre- and postembolization CT scans. Serum bilirubin above $50 \mu \mathrm{mol} / \mathrm{L}$ was used as a clinical marker of cholestasis. The degree of hypertrophy (DH) as percentual increase, and kinetic growth rate (KGR) as percentage/week, were used to assess PVE-induced hypertrophy. A total of 50 patients (31 CRLM, 19 pCCA) were included. The DH and KGR were similar in patients with pCCA and CRLM. Neither bilirubin levels before biliary drainage (pCCA patients) nor before PVE (entire cohort) were correlated with $\mathrm{DH}$ or KGR. For patients with pCCA, unilateral drainage in FLR induced a higher DH than bilateral drainage (6.7 [4.9-7.9] versus 2.7 [1.5-4.2] \%, $p=0.012)$. After biliary drainage, ten patients with pCCA remained hyperbilirubinemic, but had comparable $\mathrm{DH}$ and KGR to patients with bilirubin levels below $50 \mu \mathrm{mol} / \mathrm{L}(\mathrm{DH}: 5.6$ [3.0-7.5] versus 
5.7 [2.4-7.0] \%, respectively, $p=0.806$; KGR: 1.7 [1.0-2.4] versus 1.9 [0.8-2.4] \%/week, respectively, $p=1.000)$. C-reactive protein levels before PVE were negatively correlated with $\mathrm{DH}$ and KGR in patients with pCCA (DH: $\rho=-0.539, p=0.038$; KGR: $\rho=-0.532$, $p=0.041)$. Our findings indicate that there was no influence of cholestasis on hypertrophy of the FLR in patients undergoing PVE. Unilateral drainage in FLR was associated with higher liver hypertrophy than bilateral drainage. Elevated inflammation response appears to be associated with impaired liver growth in patients with pCCA.

Automated chyme reinfusion in intestinal failure patients with a temporary double enterostomy (TDE) restores intestinal function and protects against IFALD, but the mechanisms are incompletely understood. In Chapter 6 we aimed to investigate whether beneficial effects of $C R$ relate to functional recovery of enterohepatic signaling via the bile salt-FGF19 axis. Blood samples were collected from 12 patients, 3 days before, at start, and 1, 3, 5 and 7 weeks after CR initiation. Plasma FGF19, TBS, C4, citrulline (CIT), bile salt composition, liver tests and nutritional risk indices were determined. Paired small bowel biopsies prior to $C R$ and after 21 days were taken and genes related to bile salt homeostasis and enterocyte function were assessed. CR induced an increase in plasma FGF19 and decreased C4 levels, indicating restored regulation of bile salt synthesis via endocrine FGF19 action. TBS remained unaltered during CR. Intestinal FXR was upregulated after 21 days of CR. Molar fractions of secondary and deconjugated bile salts were increased after $C R$, reflecting restored microbial metabolism of host bile salts. Furthermore, CIT and albumin levels were gradually rising after $C R$, while abnormal serum liver tests normalized after $C R$, indicating restored intestinal function, improved nutritional status and amelioration of IFALD. CR increased gene transcripts related to enterocyte number, carbohydrate handling and bile salt homeostasis. Taken together, beneficial effects of CR are partly mediated by recovery of the bile salt-FGF19 axis and subsequent homeostatic regulation of bile salt synthesis, contributing to treat/prevent IFALD.

In Chapter 7 we provided a general discussion in the context of an important role of enterohepatic bile salt signaling in liver regeneration and intestinal failure, along with a perspective view on future studies and potential implications. 




\section{Appendix II}

Impact 



\section{Relevance}

\section{Social and economic relevance}

Enterohepatic circulation (EHC) of bile salt plays an important role in gut-liver function

${ }^{1}$. However, surgical patients, especially patients with hepatobiliary malignancies, often suffer from a disrupted EHC, which has detrimental effects on gut-liver health ${ }^{2}$. For instance, patients with perihilar cholangiocarcinoma (pCCA) have an obstructed EHC, due to malignant obstruction of the bile duct. These patients can develop obstructive jaundice and cholestatic liver injury ${ }^{3}$. Furthermore, for patients undergoing partial hepatectomy, especially when accompanied by concomitant removal of the gallbladder during extended resection procedures, the EHC can be temporarily disrupted due to removal of the 'source' of bile salts. Major liver resection can lead to post-hepatectomy liver failure (PHLF) and death, especially in patients with pCCA ${ }^{4} .{ }^{55,6744}$ Likewise, patients with insufficient future liver remnant (FLR) volume suffer unfavorable high risk of PHLF and mortality after partial liver resection ${ }^{5}$. To solve this problem, the nuclear bile salt receptor farnesoid $X$ receptor (FXR) agonist obeticholic acid (OCA) has been shown to accelerate hypertrophy of FLR in experimental portal vein embolization (PVE) model ${ }^{6}$. Pharmacological treatment with OCA has promise to induce augmented FLR hypertrophy in patients scheduled for extended partial liver resection, contributing to decreased incidence of PHLF. From economic perspective, it can avoid more medical costs through decreasing postoperative morbidity.

In addition, intestinal failure (IF) is another common health issue in digestive surgery. IF patients with a temporary double enterostomy (TDE) in the jejunum have a disrupted EHC due to outflow of succus entericus in the stoma bag ${ }^{7}$. These patients require parenteral nutrition (PN) for weeks or even months ${ }^{8}$. However, $\mathrm{PN}$ is associated with various complications, such as infectious, mechanical and metabolic complications ${ }^{9}$. An important consequence of IF can be the development of intestinal failureassociated liver disease (IFALD) that is characterized by cholestasis, hepatic steatosis and fibrosis. These complications can result in hospital readmissions and increased healthcare-related costs ${ }^{7}$. Additionally, the patients' quality of life is seriously affected by IF. Chyme (intestinal or fistula secretions) reinfusion (CR), an extracorporeal enteral nutrition technique, contributed to improved nutritional status, better intestinal absorptive function, recovery from IFALD, reduced intestinal secretions and shorter period to weaning of $\mathrm{PN}$. 
Appendix II

\section{Scientific relevance}

In this thesis, we conducted several studies to investigate the role of enterohepatic bile salt signaling in compensatory liver growth and intestinal failure. Firstly, we systematically assessed the effect and route of preoperative biliary drainage (BD) in patients with resectable pCCA. Due to high risk of bias in the current available evidences, we propose future randomized prospective studies to evaluate the value of preoperative $\mathrm{BD}$ in resectable pCCA. Furthermore, we explored the suitability of human precision-cut liver slices (hPCLS) as an in vitro model to study liver regeneration. Further optimization of the procedure and/or culture conditions, and consideration of patient factors are required before (un)suitability of hPCLS as a model system can be judged. In addition, we revealed that OCA improves bile salt homeostasis after PVE in rabbits, likely through inhibiting hepatic synthesis and promoting export of bile salts. This underlies in part the accelerated hypertrophy of the non-embolized liver lobe. It is promising to further evaluate the effect of OCA in surgical patients with small FLR scheduled for (extended) hepatectomy. A large body of safety data with OCA is available from phase III and ongoing phase IV trials in patients with chronic cholestatic and metabolic liver diseases ${ }^{10-12}$. Finally, through analyzing bile salt-FGF19 signaling in IF patients treated with chyme reinfusion, we revealed that chyme reinfusion restored EHC of bile salt and bile salt-FGF19 signaling, which partly contributed to improved gut-liver function in this patient population.

\section{Target groups}

The studies performed in this thesis provide novel knowledge and perspectives for the scientific community. We demonstrated that OCA improved bile salt homeostasis, and this in part contributes to accelerated hypertrophy of the non-embolized liver lobe after PVE in rabbits. These observations can inspire scientists and clinicians to explore the use of pharmacological activation of FXR to accelerate PVE-induced liver growth in human studies.

Meanwhile, insights form the studies described here can also benefit clinical doctors. Given the importance of the EHC of bile salts in daily practice, nutritionists can consider chyme reinfusion as first-line treatment in IF patients with a TDE. This can also improve/prevent some PN-related complications. In addition, because internal biliary 
drainage can restore EHC of bile salt, physicians can consider this potential benefit when choosing a drainage method in patients with biliary obstruction.

The ultimate aim of medical research is to improve the health of humans. Our findings may contribute to improve outcomes of surgical patients, including patients planned for (extended) liver partial resection, and IF patients with TDE.

\section{Innovation and Implementation}

In this thesis, we investigated the link between enterohepatic bile salt signaling and liver regeneration and intestinal failure. For the first time, a systematic review of systematic reviews was conducted to assess the effect and route of preoperative BD in patients with resectable pCCA and indicated a high risk of bias in current available systematic reviews. Additionally, although our previous study ${ }^{6}$ showed that OCA promoted liver regeneration following PVE in a rabbit model, the mechanism was not clarified. Our study indicated that improved bile salt homeostasis contributed to accelerated liver growth induced by PVE in OCA-treated animals. Furthermore, for IF patients with TDE, the RESCUE study revealed that restored enterohepatic bile saltFGF19 signaling underlies in part the beneficial effects of chyme reinfusion on gut-liver function, thus reversing IFALD.

The effect of preoperative BD in resectable pCCA is under debate. Further studies with large sample sizes should focus on the indications for BD, subsequently determine what kind of patients with pCCA can benefit from preoperative BD. Additionally, the effectiveness of OCA for promoting liver growth has been investigated in the rabbit model of PVE. Future research to study effect of OCA on tumor growth in embolized lobes in pre-clinical setting are recommended. Moreover, in the nutritional units, chyme reinfusion (Fig. 1) should be considered as preferred treatment for IF patients with TDE to avoid potential $\mathrm{PN}$-associated morbidity and improve intestinal rehabilitation. $\mathrm{A}$ portable roller pump would allow patients to be ambulant during the pre-operative setting ${ }^{13}$. 
Appendix II

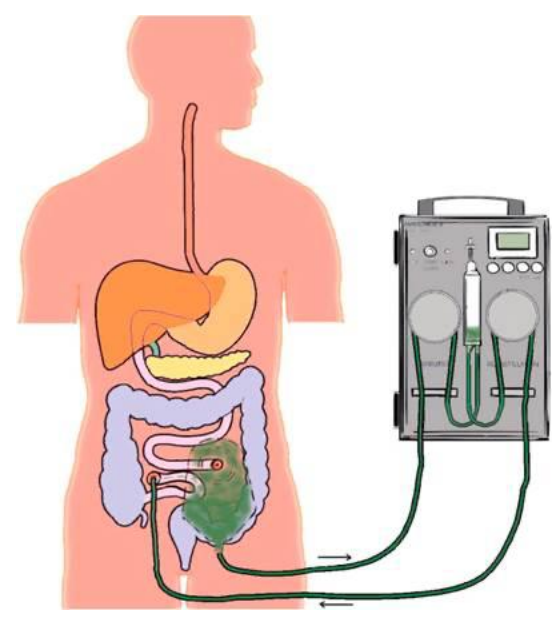

Figure 1. Chyme reinfusion technique with the automated roller pumps. Continuous chyme reinfusion was performed in a closed system of extra-corporal circulation of chyme using the Enteromate $\|{ }^{\circledR}$ system (Labodial, Clayes-sous-Bois, France). The left pump works permanently and aspirates the jejunal effluent from the stoma bag toward a plastic container. When the minimal volume of about $10 \mathrm{ml}$ in the container is exceeded, the second pump starts and the contents are infused into the downstream small intestine. This illustration is a modification from a picture of Picot et al. (2017) ${ }^{7}$. 


\section{References}

1. Schaap FG, Trauner M, Jansen PL. Bile acid receptors as targets for drug development. Nat Rev Gastroenterol Hepatol. 2014;11:55-67.

2. Hegyi P, Maléth J, Walters JR, Hofmann AF, Keely SJ. Guts and Gall: Bile Acids in Regulation of Intestinal Epithelial Function in Health and Disease. Physiol Rev. 2018;98:1983-2023.

3. Valle JW, Kelley RK, Nervi B, Oh DY, Zhu AX. Biliary tract cancer. Lancet. 2021;397:428-44.

4. van Mierlo KM, Schaap FG, Dejong CH, Olde Damink SW. Liver resection for cancer: New developments in prediction, prevention and management of postresectional liver failure. $\mathrm{J}$ Hepatol. 2016;65:1217-31.

5. Olthof PB, Wiggers JK, Groot Koerkamp B, Coelen RJ, Allen PJ, Besselink MG, et al. Postoperative Liver Failure Risk Score: Identifying Patients with Resectable Perihilar Cholangiocarcinoma Who Can Benefit from Portal Vein Embolization. J Am Coll Surg. 2017;225:387-94.

6. Olthof PB, Huisman F, Schaap FG, van Lienden KP, Bennink RJ, van Golen RF, et al. Effect of obeticholic acid on liver regeneration following portal vein embolization in an experimental model. Br J Surg. 2017;104:590-9.

7. Picot D, Layec S, Dussaulx L, Trivin F, Thibault R. Chyme reinfusion in patients with intestinal failure due to temporary double enterostomy: A 15-year prospective cohort in a referral centre. Clin Nutr. 2017;36:593-600.

8. Pironi L, Arends J, Baxter J, Bozzetti F, Pelaez RB, Cuerda C, et al. ESPEN endorsed recommendations. Definition and classification of intestinal failure in adults. Clin Nutr. 2015;34:171-80.

9. Hartman C, Shamir R, Simchowitz V, Lohner S, Cai W, Decsi T, et al. ESPGHAN/ESPEN/ESPR/CSPEN guidelines on pediatric parenteral nutrition: Complications. Clin Nutr. 2018;37:2418-29.

10. Hirschfield GM, Mason A, Luketic V, Lindor K, Gordon SC, Mayo M, et al. Efficacy of obeticholic acid in patients with primary biliary cirrhosis and inadequate response to ursodeoxycholic acid. Gastroenterology. 2015;148:751-61.e8.

11. Kjærgaard K, Frisch K, Sørensen M, Munk OL, Hofmann AF, Horsager J, et al. Obeticholic acid improves hepatic bile acid excretion in patients with primary biliary cholangitis. $J$ Hepatol. 2021;74:58-65.

12. Neuschwander-Tetri BA, Loomba R, Sanyal AJ, Lavine JE, Van Natta ML, Abdelmalek MF, et al. Farnesoid $X$ nuclear receptor ligand obeticholic acid for non-cirrhotic, non-alcoholic steatohepatitis (FLINT): a multicentre, randomised, placebo-controlled trial. Lancet. 2015;385:956-65.

13. Thibault R, Picot D. Chyme reinfusion or enteroclysis in nutrition of patients with temporary double enterostomy or enterocutaneous fistula. Curr Opin Clin Nutr Metab Care. 2016;19:382-7. 



\section{Appendix III}

Acknowledgements 

The China Scholarship Council (CSC) is acknowledged for supporting my PhD study for four years in the Department of Surgery, Maastricht University in the Netherlands. Also, I would like to express my gratitude to many people for their support and encouragement during my $\mathrm{PhD}$ and writing this thesis.

First and foremost, I would like to express my sincere gratitude to my promotor Prof. Steven Olde Damink. Steven, thanks for providing me the opportunity to have my PhD study in such a fantastic research group in the Netherlands. Many thanks for your time in our first video call, which is the outset of my PhD study. Now, four years passed by, I learned a lot from your supervision. Thanks for your guidance on my research, for instance, how to propose a research question and design a study. Thanks for teaching me how to give the presentation in research meetings. I remembered that you provided me timely feedback on my first presentation, which is so helpful for me. Also, thanks a lot for helping me in a short time when I have research questions or difficulties during work and life. I am very impressed with how you deal with one hundred things in such an efficient way. Additionally, thanks for organizing the barbecue every year in your super big garden. I really enjoyed our communications around the bonfire, such as your advice on my future work. You are such a fantastic promotor. I dream to be a surgeon and a researcher like you.

Frank, thank you so much for your daily supervision and support. I learned a lot from you during my $\mathrm{PhD}$ study. Under your supervision, I mastered a lot of practical research skills, data management skills, scientific thinking, and academic writing, etc. You are a great scientist. In the weekly meetings, I always learned a great deal from you. For instance, you told me that I should learn how to ask research questions. Your comments on how to improve posters, manuscripts, and presentations in detail are so helpful for my future career. I will apply your strict standard to every work I will do. Thanks for always being there for my (research) questions. Thanks for your encouragement during my PhD study.

I would like to express my sincere thanks to colleagues who contributed to my research. Many thanks to Kiran Koelfat, Denis Picot, Ronan Thibault, Remon Korenblik, Bram Olij, Ronald van Dam, Cornelis Dejong, Bas Groot Koerkamp, Christiaan van der Leij, Ulf Neumann, Jos Kleijnen, Cathy van Himbeeck, Pim Olthof, Han Jin, Zita Soons, Rowan van Goolen, Peter Jansen, Thomas van Gulik, Michal Heger, Bob Knapen, Lloyd Brandts, Daniel Heise, Marcel den Dulk, Sander van Kuijk, Martin Lenicek, 
Sabrina Layec, Marie Carsin, Laurence Dussaulx, Eloi Seynhaeve, Florence Trivin, Laurence Lacaze. Thanks for all your input, suggestions, and discussions on the studies and manuscripts.

Thanks to all of the members of the Department of Surgery, I received so much support. I really enjoyed being a member of this nice department. All of you always help and encourage me, which makes me feel at home. Many thanks to Bas, Mo, Hans, Annemarie, Chantal, Loes for your technical support. Ralph, thanks for helping me with the clinical data. Many thanks to my roommates Hong, Rob. I am so happy of working together with you. Thanks for taking care of me during my PhD study in the Netherlands. Many thanks to Lin, Min, Xiuxiang, Mirjam, Merel, Rianne, Annet, Kees, Janine, Anne, Romy, Sanaz, and all PhD students. It is so nice to join the weekly research meetings, share our research, celebrate Sinterklaas day, and have beautiful research days together.

I would like to express many thanks to my Chinese friends Han Jin, Shenghua Zong, Lingling Ding, Jianhua Cao, Chang Lu, Ying Gong, Yiwen Yu, Ying Xin, Qi Qiao, Le Guo, Shuo Zhang, and all my friends. I enjoyed sharing experiences, drinking, having dinner, and playing games together with you. Also, I love playing basketball with you guys during the weekend. Those activities we had during my PhD study always made me go back to work with full of energy. For many of you, we come from the same province. It is so nice to share our feelings, difficulties, solutions of research and discussion on future work and life together. Thank you for all your friendship that makes me feel relaxed and warm.

I would like to thank my family for your support. Dear father, mother, my sister and my brother in law, thank you very much for your warm care within these years. Thanks for always being there with me. You always fully support my life choice with no judgment. Every time when I face challenges, you give me a lot of strength. Best wishes for all of you.

Hongxia, my dear girlfriend, thanks for coming to the Netherlands with me to conduct our PhD studies. Even though we are not in the same university, not in the same city, we still always care about each other. The most exciting thing for me is going to Leiden to see you by train every two weeks. We together celebrated the New year, and other traditional Chinese festivals. We went to many countries in Europe. Thanks for your accompany with me for all the time. 




\section{Appendix IV}

Curriculum Vitae 

Xinwei Chang was born on the November $28^{\text {th }}$, 1988 in Henan, China. In September of 2009, he started his study on Clinical Medicine at Nanchang University and received his bachelor's degree in July of 2014. Later on, in September of 2017, he started his master's study on Surgery at Zhengzhou University. Her master thesis was entitled: "TERT promoter mutation in hepatocellular carcinoma and renal pelvic carcinoma". Under the supervision of Prof. Xiuxian Ma, he obtained his master's degree in July of 2017.

In September of 2017, he was awarded a national grant from the China Scholarship Council and started his PhD study at the Department of Surgery of Maastricht University. His research was supervised by Prof. Steven Olde Damink and Dr. Frank Schaap. During his PhD study, he focused on the role of bile salt-FGF19 signaling in promoting liver regeneration and improving intestinal failure. The results of his PhD studies are presented in this thesis. He was the member of European Association for the Study of the Liver and is also a member of Dutch Society of Hepatology. In 2019, he received a seeding grant from Division 2 of School of Nutrition and Translational Research in Metabolism in Maastricht University. In 2020, he was awarded a young investigator bursary in the International Liver Congress. After his PhD graduation in 2021, he will move back to China and start his clinical training in surgery. 



\section{Appendix V}

List of publications 



\section{List of publications}

1. Koelfat KVK ${ }^{*}$, Picot $D^{*}$, Chang $\mathbf{X}^{*}$, Desille M, van Eijk HM, van Kuijk SMJ, Lenicek M, Layec S, Carsin M, Dussaulx L, Seynhaeve E, Trivin F, Lacaze L, Thibault $R^{* *}$, Schaap $\mathrm{FG}^{* *}$, Olde Damink SWM ${ }^{* *}$. Chyme reinfusion restores the regulatory bile salt-FGF19 axis in intestinal failure patients. Hepatology 2021, doi: 10.1002/hep.32017. ("Authors share co-first authorship, "*Authors share co-senior authorship)

2. Chang X, Schaap FG, Shen H, Dejong CHC, Groot Koerkamp B, van der Leij C, Neumann UP, Kleijnen J, Olde Damink SWM. The effect and route of preoperative biliary drainage in patients with resectable perihilar cholangiocarcinoma. (submitted)

3. Chang X, van Himbeeck C, Olthof PB, Jin H, Soons Z, van Eijk HM, van Goolen R, Jansen PL, van Gulik T, Heger M, Olde Damink SWM, Schaap FG. Obeticholic acid accelerates portal vein embolization-induced liver hypertrophy by improving bile salt homeostasis in rabbits. (submitted)

4. Chang $\mathbf{X}^{\star}$, Korenblik $\mathrm{R}^{\star}$, Olij B, Knapen B, van der Leij C, Brandts L, Heise D, den Dulk M, Neumann UP, Schaap FG, van Dam R, Olde Damink SWM. No influence of cholestasis on portal vein embolization-induced hypertrophy of the future liver remnant. ('Authors share co-first authorship) (submitted)

5. Shen H, van der Kleij R, van der Boog PJM, Chang X, Wang W, Song X, Li Z, Lou $X$, Chavannes $N$. Prevalence of reduced kidney function, kidney function decline and related risk factors among a primary care population in China: A repeated crosssectional study. (submitted)

6. Shen H, van der Kleij R, van der Boog PJM, Chang X, Chavannes NH. Electronic Health Self-Management Interventions for Patients With Chronic Kidney Disease: Systematic Review of Quantitative and Qualitative Evidence. Journal of Medical Internet Research 2019, 21(11):e12384.

7. Chang $\mathbf{X}^{\star}$, Shen $\mathrm{H}^{*}$, Li, J, Qiao Z, Wan J, Ma X. Association between adiponectin levels and cardiovascular disease risk in dialysis patients: a meta-analysis. International Journal of Clinical and Experimental Medicine 2016, 9(10): 1885318859. ('Authors share co-first authorship)

8. Shen $\mathrm{H}^{*}$, Chang $\mathbf{X}^{*}$, Zheng X, Du W, Zhao B, Wang W, Lou X. Association between 
Appendix V

C-reactive protein and mortality in peritoneal dialysis patients: a meta-analysis. International Journal of Clinical and Experimental Medicine 2016, 9(9): 17579-17587. ('Authors share co-first authorship)

9. Chang X, Ma X, Shen H, Li J, Huang X. Expression of Engrailed-2 in hepatocellular carcinoma and effect of silencing Engrailed-2 gene on the proliferation and invasion of hepatocellular carcinoma cells. Journal of Practical Medicine 2017, 33(4): 82-85.

10. Chang $\mathbf{X}$, Ma $X$, Zhu $R$, Li J. Imaging evaluation of residual cancer after radiofrequency ablation of hepatocellular carcinoma. World Chinese Journal of Digestology 2016, 24(10): 1539-1544.

11. Chang X, Ma X, Shen H, Li J, Qiao Z, Wan J. Clinical analysis of 12 patients with sarcomatoid carcinoma of the gallbladder. World Chinese Journal of Digestology 2016, 24(13): 2063-2069.

\section{Oral presentations}

1. May, 2021: Dutch Translational Metabolism Conference-Seminar 4 (online). Chyme reinfusion restores the regulatory bile salt-FGF19 axis in intestinal failure patients

2. November, 2020: NUTRIM Symposium (online). The effect of chyme reinfusion (CR) on the bile salt/fibroblast growth factor 19 (FGF19) signaling in intestinal failure patients with a temporary jejunal double enterostomy: RESCUE study

3. August, 2020: The International Liver Congress (online). The effect of chyme reinfusion on the bile salt/fibroblast growth factor 19 signaling in intestinal failure patients with a temporary jejunal double enterostomy

4. November, 2019: $16^{\text {th }}$ International Medical Postgraduate Conference (Hradec Králové, Czech Republic). The effect of chyme reinfusion on the bile salt/FGF19 signaling axis in intestinal failure patients with a temporary jejunal double enterostomy

\section{Poster presentations}

- August, 2020: The International Liver Congress (online). Preoperative biliary drainage in perihilar cholangiocarcinoma: an overview of systematic reviews 

